\title{
The 2018 European Heart Rhythm Association Practical Guide on the use of non-vitamin K antagonist oral anticoagulants in patients with atrial fibrillation
}

\author{
Jan Steffel ${ }^{1} *$, Peter Verhamme ${ }^{2}$, Tatjana S. Potpara ${ }^{3}$, Pierre Albaladejo ${ }^{4}$, \\ Matthias Antz ${ }^{5}$, Lien Desteghe ${ }^{6}$, Karl Georg Haeusler ${ }^{7}$, Jonas Oldgren ${ }^{8}$, \\ Holger Reinecke ${ }^{9}$, Vanessa Roldan-Schilling ${ }^{10}$, Nigel Rowell ${ }^{11}$, Peter Sinnaeve ${ }^{2}$, \\ Ronan Collins $^{12}$, A. John Camm ${ }^{13}$, and Hein Heidbüchel ${ }^{6,14}$
}

Advisors: Martin van Eickels, M.D. (Bayer Healthcare), Jutta Heinrich-Nols, M.D. (Boehringer Ingelheim), Markus Müller, M.D., Ph.D. (Pfizer), Wolfgang Zierhut M.D. (Daiichi-Sankyo) and Poushali Mukherjea, Ph.D. (Bristol-Myers Squibb)

Document reviewers (ESC scientific document group): Gregory YH Lip (EHRA Review Coordintaor; UK, Denmark), Jeffrey Weitz (Canada), Laurent Fauchier (France), Deirdre Lane (UK), Giuseppe Boriani (Italy), Andreas Goette (Germany), Roberto Keegan (Argentina), Robert MacFadyen (Australia), Chern-En Chiang (Taiwan), Boyoung Joung (Korea), and Wataru Shimizu (Japan)

\footnotetext{
${ }^{1}$ Department of Cardiology, University Heart Center Zurich, Rämistrasse 100, CH-8091 Zurich, Switzerland; ${ }^{2}$ Department of Cardiovascular Sciences, University of Leuven, Leuven, Belgium; ${ }^{3}$ School of Medicine, Belgrade University, Belgrade, Serbia; ${ }^{4}$ Grenoble-Alps University Hospital, Grenoble, France; ${ }^{5}$ City Hospital Braunschweig, Braunschweig, Germany; ${ }^{6}$ Faculty of Medicine and Life Sciences, Hasselt University, Hasselt, Belgium; ${ }^{7}$ Center for Stroke Research Berlin and Department of Neurology, CharitéUniversitätsmedizin Berlin, Berlin, Germany; ${ }^{8}$ Uppsala Clinical Research Center and Department of Medical Sciences, Uppsala University, Uppsala, Sweden; ${ }^{9}$ Department of Cardiovascular Medicine, University Hospital Münster, Münster, Germany; ${ }^{10}$ University of Murcia, Murcia, Spain; ${ }^{11}$ Middlesbrough, UK; ${ }^{12}$ Age-Related Health Care \& StrokeService, Tallaght Hospital, Dublin Ireland; ${ }^{13}$ Cardiology Clinical Academic Group, Molecular \& Clinical Sciences Institute, St George's University, London, UK, and Imperial College; and ${ }^{14}$ Antwerp University and University Hospital, Antwerp, Belgium
}

The current manuscript is the second update of the original Practical Guide, published in 2013 [Heidbuchel et al. European Heart Rhythm Association Practical Guide on the use of new oral anticoagulants in patients with non-valvular atrial fibrillation. Europace 2013;15:625-651; Heidbuchel et al. Updated European Heart Rhythm Association Practical Guide on the use of non-vitamin K antagonist anticoagulants in patients with non-valvular atrial fibrillation. Europace 2015;17:1467-1507]. Non-vitamin K antagonist oral anticoagulants (NOACs) are an alternative for vitamin $\mathrm{K}$ antagonists (VKAs) to prevent stroke in patients with atrial fibrillation (AF) and have emerged as the preferred choice, particularly in patients newly started on anticoagulation. Both physicians and patients are becoming more accustomed to the use of these drugs in clinical practice. However, many unresolved questions on how to optimally use these agents in specific clinical situations remain. The European Heart Rhythm Association (EHRA) set out to coordinate a unified way of informing physicians on the use of the different NOACs. A writing group identified 20 topics of concrete clinical scenarios for which practical answers were formulated, based on available evidence. The 20 topics are as follows i.e., (1) Eligibility for NOACs; (2) Practical start-up and follow-up scheme for patients on NOACs; (3) Ensuring adherence to prescribed oral anticoagulant intake; (4) Switching between anticoagulant regimens; (5) Pharmacokinetics and drug-drug interactions of NOACs; (6) NOACs in patients with chronic kidney or advanced liver disease; (7) How to measure the anticoagulant effect of

* Corresponding author. Tel: +41 4425515 15, Fax: +41 44255 8701, Email: j.steffel@gmx.ch

Published on behalf of the European Society of Cardiology. All rights reserved. (c) The Author(s) 2018. For permissions, please email: journals.permissions@oup.com. 
NOACs; (8) NOAC plasma level measurement: rare indications, precautions, and potential pitfalls; (9) How to deal with dosing errors; (10) What to do if there is a (suspected) overdose without bleeding, or a clotting test is indicating a potential risk of bleeding; (11) Management of bleeding under NOAC therapy; (12) Patients undergoing a planned invasive procedure, surgery or ablation; (13) Patients requiring an urgent surgical intervention; (14) Patients with AF and coronary artery disease; (15) Avoiding confusion with NOAC dosing across indications; (16) Cardioversion in a NOAC-treated patient; (17) AF patients presenting with acute stroke while on NOACs; (18) NOACs in special situations; (19) Anticoagulation in AF patients with a malignancy; and (20) Optimizing dose adjustments of VKA. Additional information and downloads of the text and anticoagulation cards in different languages can be found on an EHRA website (www.NOACforAF.eu).

\section{Abbreviations}

ACS
ACT
AF
AMPLIFY

ANNEXA

aPCC

aPTT ARISTOTLE

ATLAS ACS-TIMI

AUGUSTUS

AXAFA-AFNET

BMI

BMS

BRIDGE

\section{CAD}

CKD

COMPASS

\section{$\mathrm{CrCl}$}

DAPT

DES

dTT

ECA

EHRA
Acute Coronary Syndrome,

Activated Clotting Time,

Atrial Fibrillation,

Apixaban for the Initial Management of

Pulmonary Embolism and Deep-Vein

Thrombosis as First-Line Therapy,

Andexanet Alfa, a Novel Antidote to the

Anticoagulation Effects of FXA Inhibitors

study,

Activated Prothrombin Complex

Concentrates,

Activated Prothrombin Time,

Apixaban for Reduction in Stroke and

Other Thromboembolic Events in Atrial

Fibrillation,

Anti-Xa Therapy to Lower Cardiovascular Events in Addition to Standard Therapy in Subjects with Acute Coronary Syndrome - Thrombolysis in Myocardial Infarction, Apixaban Versus Vitamin K Antagonist in Patients With Atrial Fibrillation and Acute Coronary Syndrome and/or Percutaneous Coronary Intervention,

Anticoagulation using the direct factor $\mathrm{Xa}$ inhibitor apixaban during Atrial Fibrillation catheter Ablation: Comparison to vitamin $\mathrm{K}$ antagonist therapy - Atrial Fibrillation Network,

Body Mass Index,

Bare metal stent,

Bridging Anticoagulation in Patients who

Require Temporary Interruption of

Warfarin Therapy for an Elective Invasive

Procedure or Surgery,

Coronary artery disease,

Chronic kidney disease,

Cardiovascular Outcomes for People

Using Anticoagulation Strategies,

Creatinine clearance,

Dual antiplatelet therapy,

Drug-eluting stent,

Diluted thrombin time,

Ecarin chromogenic assay,

European Heart Rhythm Association,

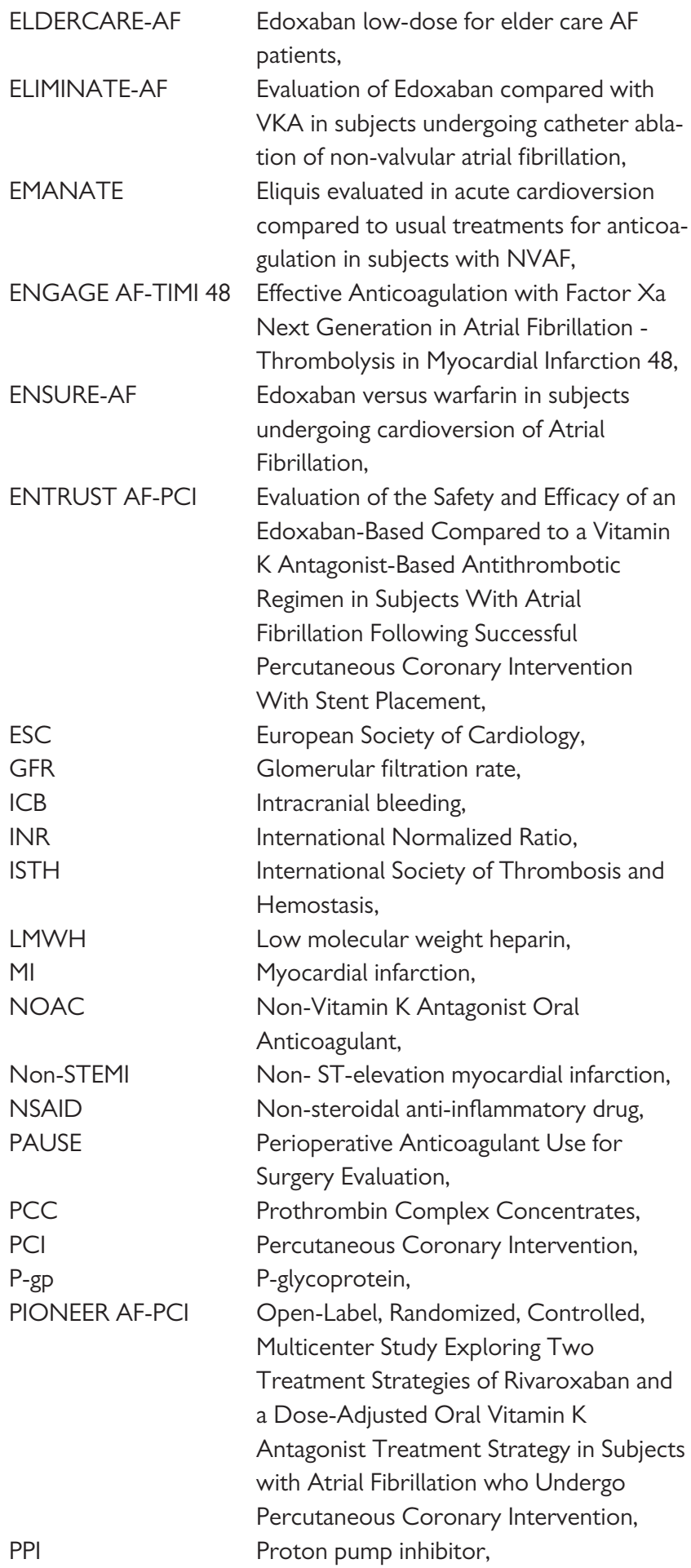


PT

RCT

RE-CIRCUIT

RE-DUAL PC

RE-LY

RE-VERSE AD

ROCKET AF

SEE

SmPC

STEMI

TIA

TT

TTR

UFH

VENTURE-AF

VHD

VKA

VTE

WOEST

$\mathrm{X}-\mathrm{VeRT}$
Prothrombin time,

Randomized clinical trial,

Randomized Evaluation of Dabigatran

Etexilate Compared to Warfarin in

Pulmonary Vein Ablation: Assessment of

an Uninterrupted Periprocedural

Anticoagulation Strategy,

Randomized Evaluation of Dual

Antithrombotic Therapy with Dabigatran versus Triple Therapy with Warfarin in

Patients with Nonvalvular Atrial

Fibrillation Undergoing Percutaneous

Coronary Intervention,

Randomized Evaluation of Long-Term

Anticoagulation Therapy,

Reversal Effects of Idarucizumab in

Patients on Active Dabigatran,

Rivaroxaban Once Daily Oral Direct

Factor Xa Inhibition Compared with

Vitamin K Antagonism for Prevention of

Stroke and Embolism Trial in Atrial

Fibrillation,

Systemic embolic event,

Summary of product characteristics,

ST-elevation myocardial infarction,

Transient ischaemic attack,

Thrombin time,

Time in therapeutic range,

Unfractionated heparin,

Active-controlled multi-center study with blind-adjudication designed to evaluate the safety of uninterrupted Rivaroxaban and uninterrupted vitamin $\mathrm{K}$ antagonists in subjects undergoing catheter ablation for non-valvular Atrial Fibrillation,

Valvular heart disease,

Vitamin K Antagonist,

Venous thromboembolic event,

What is the Optimal antiplatelet and anticoagulant therapy in patients with oral anticoagulation and coronary stenting, Explore the efficacy and safety of oncedaily oral rivaroxaban for the prevention of cardiovascular events in patients with non- valvular atrial fibrillation scheduled for cardioversion

\section{Introduction}

Non-vitamin K antagonist oral anticoagulants (NOACs) are an alternative for vitamin $\mathrm{K}$ antagonists (VKAs) to prevent stroke in patients with atrial fibrillation (AF) and have emerged as the preferred choice, particularly in patients newly started on anticoagulation. ${ }^{1-3}$ The term NOAC has been used for many years, is used by the current European Society of Cardiology (ESC) AF guidelines ${ }^{3}$ and is widely recognized. Therefore, even though some authors refer to these

drugs as 'direct oral anticoagulants', ${ }^{4}$ we prefer to continue to use NOAC. Ultimately, both terms are interchangeable when referring to the direct factor $\mathrm{Xa}$ inhibitors apixaban, edoxaban, and rivaroxaban as well as the direct thrombin inhibitor dabigatran.

Non-vitamin $\mathrm{K}$ antagonist oral anticoagulants have an improved efficacy/safety ratio, a predictable anticoagulant effect without need for routine coagulation monitoring, and fewer food and drug interactions compared with VKAs. However, the proper use of NOACs requires a carefully considered approach to many practical aspects. Whereas the ESC Guidelines ${ }^{3}$ mainly discuss the indications for anticoagulation in general and of NOACs in particular, they offer less guidance on how to deal with NOACs in specific clinical situations. Moreover, there are still several less well researched aspects of NOAC use, which are nonetheless relevant when these drugs are used by cardiologists, neurologists, geriatricians, general practitioners, and other healthcare providers in daily clinical practice. Each of the NOACs available on the market is accompanied by the instructions for its proper use in many clinical situations [summary of product characteristics (SmPCs); patient card; information leaflets for patients; and physicians], but multiple, and often slightly different, physician education tools sometimes create confusion rather than clarity. Based on these premises, the European Heart Rhythm Association (EHRA) set out to coordinate a unified way of informing physicians on the use of NOACs. The first edition of the Practical Guide was published in 2013 to supplement the AF guidelines as guidance for safe and effective use of NOACs when prescribed; a first update was published in 2015. ${ }^{1,2}$ This text is the 2018 update to the original Guide.

A writing group formulated practical answers to 20 clinical scenarios, based on available and updated knowledge. The writing group was assisted by medical experts from the manufacturers of the NOACs, who provided assurance that the latest information on the different NOACs was evaluated, and provided feedback on the alignment of the text with the approved SmPCs. However, the final responsibility of this document resided entirely with the EHRA writing group. In some instances, the authors opted to make recommendations that do not fully align with all SmPCs, with the goal to provide more uniform and simple practical advice (e.g. on the start of NOAC after cessation of VKA, on advice after a missed or forgotten dose, on perioperative management, and others).

An EHRA website, www.NOACforAF.eu, accompanies the Practical Guide. The Practical Guide is summarized in a Key Message booklet which can be obtained through EHRA and ESC; the website also provides EHRA members with a downloadable slide kit on the Practical Guide.

We hope that with the current revision the practical tool that EHRA envisioned has further improved. The authors realize that there will be gaps, unaddressed questions, and areas of uncertainty and debate. Therefore, readers can address their suggestions for change or improvement on the website. This whole endeavour should be one for and by the medical community with the ultimate goal of improving patient care and outcome.

\section{Eligibility for non-vitamin $K$ antagonist oral anticoagulants}

Non-vitamin K antagonist oral anticoagulants are approved for stroke prevention in non-valvular atrial fibrillation. Strictly, the term "non- 
Table I Selected indications and contraindications for non-vitamin K antagonist oral anticoagulant therapy in atrial fibrillation patients

\begin{tabular}{|c|c|}
\hline Condition & Eligibility for NOAC therapy \\
\hline Mechanical prosthetic valve & Contraindicated \\
\hline $\begin{array}{l}\text { Moderate to severe mitral stenosis } \\
\text { (usually of rheumatic origin) }\end{array}$ & Contraindicated \\
\hline $\begin{array}{l}\text { Mild to moderate other native valvular } \\
\text { disease (e.g., mild-moderate aortic } \\
\text { stenosis or regurgitation, degenerative } \\
\text { mitral regurgitation etc.) }\end{array}$ & Included in NOAC trials \\
\hline Severe aortic stenosis & $\begin{array}{l}\text { Limited data (excluded in RE-LY) } \\
\text { Most will undergo intervention }\end{array}$ \\
\hline \multirow{2}{*}{$\begin{array}{l}\text { Bioprosthetic valve (after }>3 \text { months } \\
\text { post operatively) }\end{array}$} & Not advised if for rheumatic mitral stenosis \\
\hline & $\begin{array}{l}\text { Acceptable if for degenerative mitral } \\
\text { regurgitation or in the aortic position }\end{array}$ \\
\hline $\begin{array}{l}\text { Mitral valve repair (after }>3 \text { months } \\
\text { post operatively) }\end{array}$ & Some patients included in some NOAC trials \\
\hline PTAV and TAVI & $\begin{array}{l}\text { No prospective data yet } \\
\text { May require combination with single or dual } \\
\text { antiplatelet therapy }\end{array}$ \\
\hline Hypertrophic cardiomyopathy & Few data, but patients may be eligible for NOACs \\
\hline
\end{tabular}

Hatched-limited data.

PTAV, percutaneous transluminal aortic valvuloplasty; TAVI, transcatheter aortic valve implantation.

valvular AF' refers to AF in the absence of a mechanical prosthetic heart valve or moderate to severe mitral stenosis (usually of rheumatic origin) (Table 1), ${ }^{3,5,6}$ which were exclusion criteria for all Phase III NOAC vs. warfarin trials in AF. In order to avoid confusion, the term 'non-valvular' has been eliminated in the 2016 ESC guidelines on the management of patients with $A F$, and reference is made to the specific underlying valvular heart disease (VHD). ${ }^{3,6}$ However, the term is still found in the individual SmPCs of each of the NOACs due to the original wording used in the exclusion criteria of the clinical trials on which their regulatory approval was based.

Based on these new developments, a novel classification has recently been suggested where a functional EHRA (Evaluated Heartvalves, Rheumatic or Artificial) categorization is proposed, depending on the type of OAC use in patients with $A F^{6}{ }^{6}$ In this scheme, EHRA Type 1 refers to $A F$ patients with $V H D$ needing therapy with a vitamin $K$ antagonist (VKA), including in particular moderate-severe mitral stenosis of rheumatic origin and mechanical prosthetic valve replacement. In contrast, EHRA Type 2 valvular heart disease refers to VHD patients needing thromboembolic prevention therapy for AF with a VKA or a NOAC, including essentially all other native valvular stenoses and insufficiencies as well as mitral valve repair, bioprosthetic valve replacements and transaortic valve intervention (TAVI). ${ }^{6}$ Patients with EHRA Type 2 valvular heart disease were variously included in these trials and NOACs demonstrated a comparable relative efficacy and safety vs. warfarin in patients with vs. without valvular disease, except for a higher risk of bleeding with rivaroxaban vs. warfarin in patients with valvular heart disease in a post hoc analysis of the ROCKET-AF trial. ${ }^{6-12}$ Non-vitamin $\mathrm{K}$ antagonist oral anticoagulants may therefore be used in such patients (Table 1). ${ }^{3,6,13}$
Atrial fibrillation in patients with biological valves or after valve repair constitute a grey area, even though these patients were included in some of the landmark NOAC trials. ${ }^{6,7,9,10}$ Since most of these patients do not require long-term oral anticoagulation following their valve procedure, the use of a NOAC for the management of concomitant $A F$ is considered to be a valid option. One exception may be $A F$ in the presence of a biological mitral prosthesis implanted for rheumatic mitral stenosis. Although mitral valve flow is normalized post-mitral valve replacement in these patients, their atria usually remain large and severely diseased. As such, VKA may be the preferred option over NOACs in these patients, but more data are needed.

There are no prospective data available yet on NOACs in patients after percutaneous aortic valve interventions [percutaneous transluminal aortic valvuloplasty or transcatheter aortic valve implantation (TAVI)] in the presence of AF. Percutaneous transluminal aortic valvuloplasty or TAVI usually requires single or even transient dual antiplatelet therapy (DAPT). ${ }^{5}$ The addition of an anticoagulant increases the bleeding risk, and the optimal combination and duration is the subject of ongoing studies, in analogy to the situation in acute coronary syndrome (ACS) patients (see chapter 14).

In hypertrophic (obstructive) cardiomyopathy (HCM), AF is associated with a high rate of thromboembolism. There is limited experience with NOACs in this condition. ${ }^{14,15}$ In contrast to patients with $\mathrm{AF}$ in the setting of mechanical valves or rheumatic mitral stenosis, however, there does not seem to be a mechanistic rationale why NOACs should be inferior to warfarin in $\mathrm{HCM}$. On the contrary, AF in HCM shares many similarities of HFpEF related $A F$, for which there has been no indication that NOAC would be inferior to VKA. ${ }^{16-18}$ Moreover, NOACs 
demonstrate a sustained efficacy over VKA also in other high risk subgroups (e.g. patients with a high $\mathrm{CHA}_{2} \mathrm{DS}_{2}$-VASC score). As such, patients with $\mathrm{HCM}$ may be eligible for NOAC therapy.

\section{Practical start-up and follow-up scheme for patients on non-vitamin $\mathrm{K}$ antagonist oral anticoagulants}

\section{Choice of anticoagulant therapy and initiation \\ Indication for anticoagulation and choice between vitamin $\mathrm{K}$ antagonist and non-vitamin $\mathrm{k}$ antagonist oral anticoagulant}

Before prescribing a NOAC to a patient with $A F$, it needs to be decided that anticoagulation is indicated based on a risk/benefit analysis. ${ }^{3}$ The choice of anticoagulant (VKA or NOAC; choice of NOAC) should be made on the basis of indications approved by regulatory authorities and specified within guidelines from professional societies. Knowledge of kidney function is required, since all NOACs have precautions and contraindications based on creatinine clearance $(\mathrm{CrCl})$ (see chapter 6). Also product characteristics (as explained in the SmPCs), patient-related clinical factors, and patient preference need to be taken into account. $3,19,20$

European guidelines have expressed a preference for NOACs over VKA in stroke prevention for AF patients, especially if newly initiated. This recommendation (Class I, level of evidence $A$ ) is based on the overall clinical benefit of NOACs. ${ }^{3}$

In some countries, NOAC therapy can only be prescribed (and/or are reimbursed) if international normalized ratio (INR) control with VKA has been shown to be suboptimal (i.e. after a failed 'trial of VKA'). There is evidence that clinical scores such as SAMe-TT ${ }_{2} R_{2}$ may be able to predict poor INR control. ${ }^{21-27}$ However, further prospective studies would be required to validate and implement such strategies (which are not generally needed from a medical perspective, but may be a reasonable cost containment strategy). For the majority of patients, and in accordance with current ESC guidelines, NOACs need to be considered as the first choice anticoagulation based on the positive results of the large outcome trials. ${ }^{3,28-31}$

\section{Choosing the type and dose of non-vitamin $\mathrm{K}$ antagonist oral anticoagulant}

With four NOACs available in different dosages for different indications and with different dose reduction criteria, identification of the correct dose has become more complicated and is one of the key challenges in the daily use and individualization of treatment (see chapter 15). Non-vitamin $\mathrm{K}$ antagonist oral anticoagulants do not have precisely the same rules for prescription and availability in every country. Local factors, such as regulatory approval, formulary committees and the cost of therapy, may influence NOAC availability.

All NOACs have been tested in large randomized prospective trials and resulted in documented efficacy and safety of the respective agent. Testing of different doses, however, was carried out differently. In ARISTOTLE (using apixaban) and ROCKET-AF (using rivaroxaban), patients received one dose which was reduced in the presence of predefined patient characteristics. ${ }^{29,30}$ In contrast, in RELY (with dabigatran) and ENGAGE-AF (with edoxaban) both a lower and a higher dose were tested in fully powered patient cohorts (with further dose reduction for edoxaban in certain patients, see chapter 15). ${ }^{28,31}$ Dose reduction of NOACs is primarily recommended only according to the dose reduction criteria investigated in the large phase III trials (see chapter 15). Whenever possible, the tested standard dose of NOACs should be used. In addition, it is also important to consider co-medications, some of which may be contraindicated or result in unfavourable drug-drug interactions (see chapter 5). Also, patient age, weight, renal function (see chapters 6 and 18), and other comorbidities influence the choice. In some patients, proton pump inhibitors (PPIs) may be considered to reduce the risk for gastrointestinal ( $\mathrm{Gl}$ ) bleeding, especially in those with a history of $\mathrm{Gl}$ bleeding or ulcer and patients requiring concomitant use of (dual) antiplatelet therapy. ${ }^{32,33}$ This gastroprotective effect was especially demonstrated in patients receiving antiplatelet or VKA therapy, ${ }^{34-36}$ while data on the preventive effects in NOAC treated patients are limited. ${ }^{37}$ Decision aids are available to guide clinicians about which NOAC may be best suited for a specific target group. ${ }^{38-}$ 41

\section{An anticoagulation card for non-vitamin $\mathrm{K}$ antagonist oral anticoagulants and the importance of education} Patients on VKAs have routinely been advised to carry information about their anticoagulant therapy to alert any healthcare provider about their treatment. It is equally important that those treated with NOACs carry details of this therapy. Each manufacturer provides proprietary information cards to be completed by physicians and carried by patients; however, we recommend that a uniform card should be used instead. The proposed NOAC card presented in this version of the Practical Guide has been updated and will be available for download in various languages at www.NOACforAF.eu.

It is critically important to educate patients at each visit about the modalities of intake [once daily (OD) or twice a day (BID); intake with food in case of $15 \mathrm{mg} / 20 \mathrm{mg}$ of rivaroxaban], the importance of strict adherence to the prescribed dosing regimen, how to deal with any lapse in dosing, and to be careful not to leave their medication behind when travelling. Key educational aspects are also listed on the NOAC anticoagulation card. Education sessions can be further facilitated using specific checklists. $3,20,42,43$

\section{How to organize follow-up?}

The follow-up of AF patients who are taking anticoagulant therapy needs to be carefully specified and communicated among the different caregivers of the patient. The use of any anticoagulant is associated with some drug-drug interactions which may increase the risk of serious bleeding or diminish stroke protection. Treatment requires vigilance due to potentially severe complications, particularly as the target patient population tends to be of older age and frail. Patients' treatment should be reviewed on a regular basis (preferably after 1 month initially and at least every 3 months thereafter). As clinical experience with NOACs grows, ${ }^{44,45}$ follow-up intervals may become longer, based on individual (patient-specific) or local (centrespecific) factors. Patient follow-up may be undertaken by general 


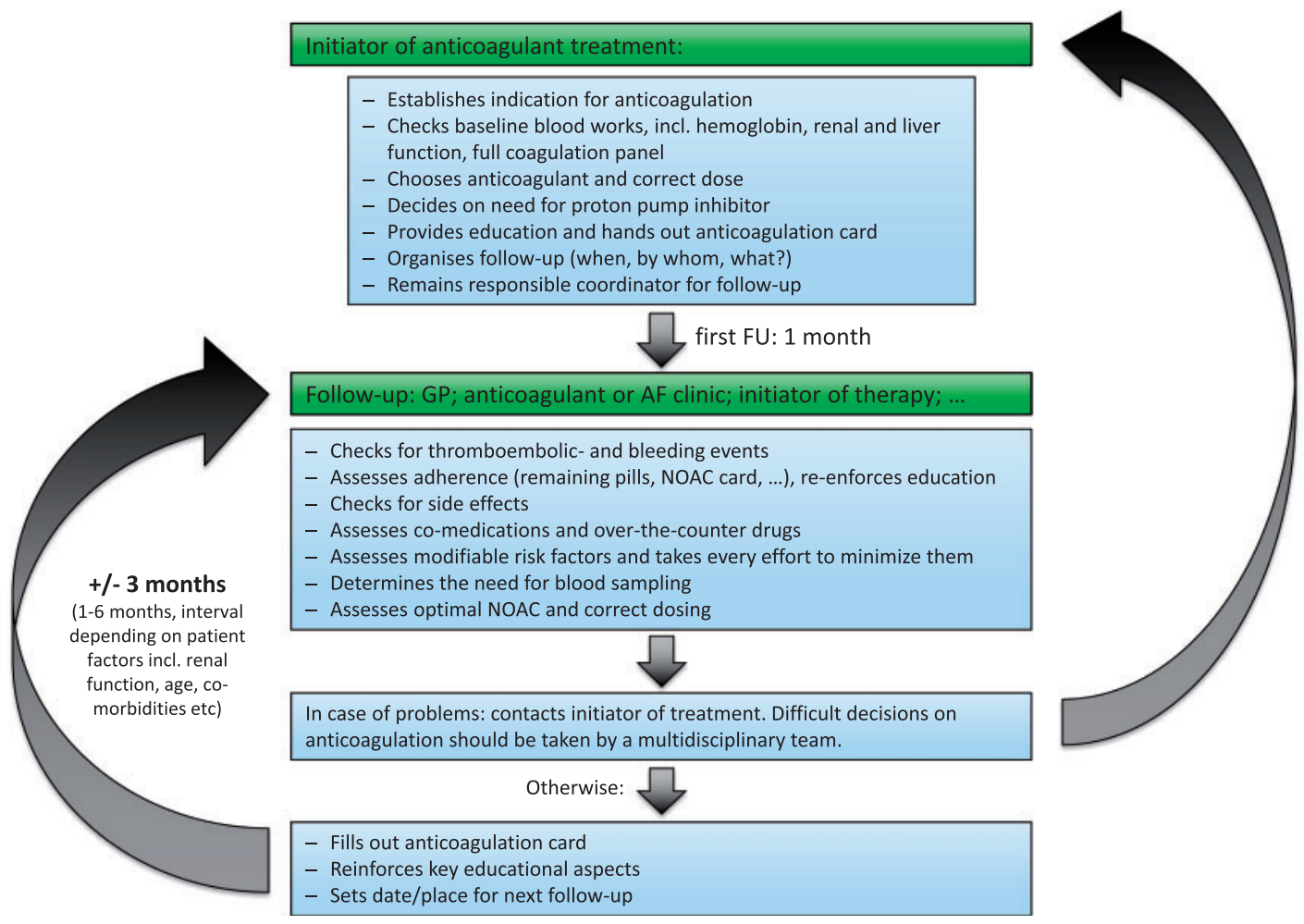

Figure I Structured follow-up. Initiation and structured follow-up of patients on non-vitamin K antagonist oral anticoagulants. It is mandatory to ensure safe and effective drug intake. The anticoagulation card is intended to document each planned visit, each relevant observation or examination, and any medication change, so that every person following up the patient is well-informed. Moreover, written communication between different healthcare providers is required to inform them about the follow-up plan and execution. FU, follow-up.

practitioners with experience in this field and/or by appropriate secondary care physicians (Figure 1). Growing evidence shows that nurse-coordinated AF clinics may be very helpful in this regard. ${ }^{46-50}$ Each caregiver, including specially trained nurses and pharmacists, should indicate with a short input on the patient NOAC card whether any relevant findings were present, and when and where the next follow-up is due.

Table 2 and Figure 1 list the appropriate timing of the relevant aspects which need to be systematically assessed during follow-up. Importantly, the individual patient profile needs to be taken into consideration; for example, renal function needs to be assessed more frequently (Table 2) in compromised individuals including older patients ( $\geq 75$ years), frail patients, ${ }^{52,53}$ or in those where an intercurrent condition (such as infection or cancer), which may affect hepatic or renal function. Also stroke risk factors alter over time and need to be reassessed at every patient visit. ${ }^{54}$ Bleeding risk schould be systematically assessed, e.g. by the HAS-BLED score, which has also been validated in patients on NOACs ${ }^{55}$ and has shown a better prediction than an approach based only on modifiable bleeding risk factors. ${ }^{56-60}$ Also other bleeding risk scores have been proposed. ${ }^{59,60}$ Importantly, however, a high bleeding risk in itself should not automatically result in the decision not to anticoagulate as stroke risk tracks along with bleeding risk. ${ }^{3,61}$ For the practical management, correcting and minimizing modifiable risk factors is of critical importance in order to minimize the risk of bleeding while on treatment with a NOAC. This approach is also the one recommended by current AF guidelines. ${ }^{3}$ Similarly, frailty and risk of falling should not generally be a reason not to anticoagulate patients, but rather to ensure careful education on the best choice of $(\mathrm{N}) \mathrm{OAC}$ and dose selection, and follow-up of the patient (see chapter 18).

\section{Ensuring adherence to prescribed oral anticoagulant intake}

Strict adherence to NOAC intake is crucial as its anticoagulant effect wanes within $12-24 \mathrm{~h}$ after the last intake. ${ }^{62}$ Non-vitamin $\mathrm{K}$ antagonist oral anticoagulant plasma level as well as general coagulation tests cannot be considered as tools to monitor adherence since they only reflect intake over the last 24(-48) h and the measured level is heavily dependent on the time between last intake and sampling (see chapter 7). The absence of a need for routine plasma level monitoring means that NOAC patients are likely to be less frequently seen for follow-up compared with VKA patients. However, there are arguments in favour of regular follow-up assessment for patients on 
Table 2 Checklist during follow-up contacts of atrial fibrillation patients on anticoagulation

\begin{tabular}{|c|c|c|}
\hline & Interval & Comments \\
\hline 1. Adherence & Each visit & $\begin{array}{l}\text { - Instruct patient to bring NOAC card and complete list of medication: make note and assess } \\
\text { average adherence } \\
\text { - Re-educate on importance of strict intake schedule } \\
\text { - Inform about adherence aids (special boxes; smartphone applications; ....). Consider specific } \\
\text { adherence measuring interventions (review of pharmacy refill data; electronic monitoring }{ }^{51} \text {; } \\
\text { special education session; ....) }\end{array}$ \\
\hline 2. Thromboembolism & Each visit & $\begin{array}{l}\text { - Systemic circulation (TIA, stroke, peripheral) } \\
\text { - Pulmonary circulation }\end{array}$ \\
\hline 3. Bleeding & Each visit & $\begin{array}{l}\text { - 'Nuisance' bleeding: preventive measures possible? Motivate patient to diligently continue } \\
\text { anticoagulation } \\
\text { - Bleeding with impact on quality-of-life or with risk: prevention possible? Need for revision of } \\
\text { anticoagulation indication, dose or timing? }\end{array}$ \\
\hline 4. Other side effects & Each visit & $\begin{array}{l}\text { Carefully assess relation with NOAC: decide for continuation (and motivate), temporary cessa- } \\
\text { tion, or change of anticoagulant drug }\end{array}$ \\
\hline 5. Co-medications & Each visit & $\begin{array}{l}\text { - Prescription drugs; over-the-counter drugs (Pharmacokinetics and drug-drug interactions of } \\
\text { non-vitamin K antagonist oral anticoagulants section). } \\
\text { - Careful interval history: also temporary use can be risky }\end{array}$ \\
\hline \multirow{4}{*}{$\begin{array}{l}\text { 6. Blood sampling } \\
\text { (incl. hemoglobin, } \\
\text { renal and liver function) }\end{array}$} & Yearly & Patients other than those specified below \\
\hline & 6-monthly & $\geq 75$ years (especially if on dabigatran) or frail (see chapter $\mathbf{2}$ ) \\
\hline & $x$-monthly & If renal function $\mathrm{CrCl} \leq 60 \mathrm{~mL} / \mathrm{min}$ : recheck interval $=\mathrm{CrCl} / 10$ \\
\hline & If needed & If intercurrent condition that may impact renal or hepatic function \\
\hline $\begin{array}{l}\text { 7. Assessing and } \\
\text { minimizing modifiable } \\
\text { risk factors for bleeding }\end{array}$ & Each visit & $\begin{array}{l}\text { - As recommended by current guidelines }{ }^{3} \\
\text { - Particularly: uncontrolled hypertension (systolic }>160 \mathrm{mmHg} \text { ), medication predisposing for } \\
\text { bleeding (e.g. aspirin, NSAIDs), labile INR (if on VKA), excessive alcohol intake) }\end{array}$ \\
\hline $\begin{array}{l}\text { 8. Assess for optimal } \\
\text { NOAC and correct } \\
\text { dosing }\end{array}$ & Each visit & $\begin{array}{l}\text { Especially based on the above, re-assess whether } \\
\text { a. The chosen NOAC is the best for the patient } \\
\text { b. The chosen dose is correct }\end{array}$ \\
\hline
\end{tabular}

For frequency of visits: see Figure 1 .

$\mathrm{CrCl}$, creatinine clearance (preferably measured by the Cockcroft-Gault method); NSAID, non-steroidal anti-inflammatory drugs; PPI, proton pump inhibitor; TIA, transient ischaemic attack.

NOACs, particularly in case of relevant co-morbidities such as renal failure, older age, multiple comorbidities, or frailty.

Available 'real world' data suggest variable adherence to NOAC intake from $38 \%$ to $99 \%$ depending on the setting and definition. ${ }^{63-78}$ Although caution is needed when interpreting these results, low adherence rates severely diminish the benefit of treatment. Some of these concerns have been alleviated by recent 'real world' implementation data which mostly confirm the improved risk/benefit profile in patients treated with NOACs vs. VKAs as observed in the randomized controlled trials suggesting adequate adherence also in daily clinical practice. ${ }^{66,72,79-98}$ Although there is evidence for significantly lower discontinuation rates with NOACs than with VKAs, discontinuation is still a relevant issue. ${ }^{67,76,77,84,95,99-107}$ Despite limited data on how NOAC adherence can best be optimized, all means possible should be considered.

\section{Practical considerations (Figure 1)}

(1) Patient education on the need for oral anticoagulation therapy and the importance of strict adherence is important. ${ }^{19,20,42,63,108-111}$ Many simultaneous approaches can be employed to provide education including leaflets and instructions at initiation of therapy, a patient anticoagulation card, group sessions, and re-education at every prescription renewal. Several organizations also offer online 
patient support websites, including EHRA (http://www.afibmatters. org/), the AF Association in the UK (http://www.atrialfibrillation.org. uk), Anticoagulation UK (www.anticoagulationuk.org), and AFNET (http://www.kompetenznetz-vorhofflimmern.de/de/vorhofflim mern/patienteninformation-vorhofflimmern). Education may be more effective if directed to specific knowledge gaps of the patient, measured by validated questionnaires which can be administered to the patient at the time of a visit, or even via online platforms. ${ }^{64,109,112}$

(2) Family members should be involved in the care of the patient, so that they understand the importance of adherence and help the patient in this regard.

(3) There should be a pre-specified follow-up schedule for the NOAC patient (as suggested in Figure 1) known to and shared by general practitioners, cardiologists, pharmacists, anticoagulation clinics, and other professionals providing care. Each of those involved has a responsibility to reinforce adherence. Everyone's efforts should be communicated to the others, e.g. by filling out a line on the NOAC anticoagulation card (see chapter 2). Nurse-coordinated AF centres may be helpful in coordinating patient follow-up and checking on adherence. ${ }^{46-50}$

(4) Some countries have a highly networked pharmacy database, which can help track the number of NOAC prescriptions that individual patients claim. In such countries, pharmacists could be involved in adherence monitoring, and this information should be used to cross-check appropriate prescription and dosing. It has been shown that an increased follow-up and adherence monitoring by pharmacists may improve NOAC adherence. ${ }^{113}$

(5) Many technological aids are being explored to enhance adherence: the day-marked blister pack format; medication boxes (conventional or with electronic verification of intake); smartphone applications ${ }^{114}$ with reminders and/or SMS messages to alert the patient about the next intake some even requiring confirmation that the dose has been taken. Popular apps for both Android and iOS devices are Medisafe Pill Reminder (also available for watchOS), Dosecast, MyMeds, CareZone, and many others. ${ }^{115}$ Again, the long-term effects of such tools are unknown and one tool may not suit all patients.

(6) Once daily dosing regimens generally results in greater adherence vs. BID regimens in cardiovascular patients. ${ }^{116-118}$ Most, but not all studies evaluating adherence for NOACs indicate that an OD dosing regimen is superior from a total tablet count perspective. ${ }^{66,67,70-74,95,112,119,120}$ However, it is still uncertain whether any regimen is superior in guaranteeing the clinical thromboembolic preventive effects and safety profile as seen in the clinical trials. ${ }^{73,83-}$ 86,90-95,121,122 Although there are modelling data suggesting that there is potentially a larger fluctuation in the anticoagulant activity when a single dose is omitted from an OD dosing regimen compared with when a single or even two doses are omitted from a BID regimen, ${ }^{123}$ the clinical relevance of these fluctuations is

unknown. ${ }^{124}$ Therefore, it is essential to ensure that drugs are taken according to the prescribed regimen.

(7) In cases where suboptimal adherence is suspected, electronic monitoring may help to educate the patient by exposing patterns of missed doses. Electronic medication intake monitoring can even be set up as a telemonitoring service, with the possibility of faster feedback to the patient. ${ }^{51}$ The health-economic validity of such an approach needs further study.
(8) Some patients may explicitly prefer INR monitoring to no monitoring, or VKA over NOAC therapy. Patient education needs to discuss these preferences in the context of available clinical trial data (including reduction in $\mathrm{ICH}$ with NOACs even in the setting of high TTR). ${ }^{19,42}$

(9) In NOAC patients in whom low adherence is suspected despite proper education and additional tools, conversion to VKAs may be considered. It needs to be kept in mind, however, that poor adherence in VKA treated patients is equally associated with INR fluctuations and less preferable outcomes.

\section{Switching between anticoagulant regimens}

When switching between different anticoagulant therapies, it is important to ensure the continuation of anticoagulant therapy while minimizing the risk for bleeding. This requires insights into the pharmaco-kinetics and -dynamics of different anticoagulation regimens, interpreted in the context of the individual patient.

\section{Vitamin $\mathrm{K}$ antagonist to non-vitamin $\mathrm{K}$ antagonist oral anticoagulant}

The NOAC can immediately be initiated once the INR is $\leq 2.0$. If the INR is 2.0-2.5, NOACs can be started immediately or (better) the next day. For INR $>2.5$, the actual INR value and the half-life of the VKA need to be taken into account to estimate the time when the INR value will likely drop to below this threshold value [half-lives for acenocoumarol 8-24h, warfarin 36-48h, phenprocoumon 120-200 h (6 days)]. The proposed scheme (also shown in Figure 2, top panel) tries to unify different specifications from the SmPCs, which state that NOAC can be started when INR is $\leq 3$ for rivaroxaban, $\leq 2.5$ for edoxaban, and $\leq 2$ for apixaban and dabigatran.

\section{Non-vitamin $\mathrm{K}$ antagonist oral anticoagulant (NOAC) and Vitamin K antagonist (VKA)}

Because of the slow onset of action of VKAs, it may take 5-10 days before the INR is in the therapeutic range, with large individual variations (see also chapter 20). Therefore, the NOAC and VKA should be administered concomitantly until the INR is in a range that is considered appropriate (Figure 2, lower panel)—similar to the situation when low molecular weight heparins (LMWHs) are administered during VKA initiation. A loading dose is not recommended for acenocoumarol and warfarin, but is appropriate with phenprocoumon (see chapter 20).

As NOACs may have an impact on INR measurements, it is important that the INR (i) is measured just before the next intake of the NOAC during concomitant administration and (ii) is re-measured early after stopping the NOAC (i.e. reflecting solely VKA therapy) to assure adequate anticoagulation. It is also recommended to closely monitor INRs within the first month until stable values have been attained (i.e. three consecutive measurements yielded values between 2.0 and 3.0). At the end of the ENGAGE-AF trial, patients on edoxaban transitioning to VKA received up to 14 days of a half dose of the NOAC until the INR was within range, in combination with the above intensive INR testing strategy. ${ }^{125}$ Switching according to this scheme 


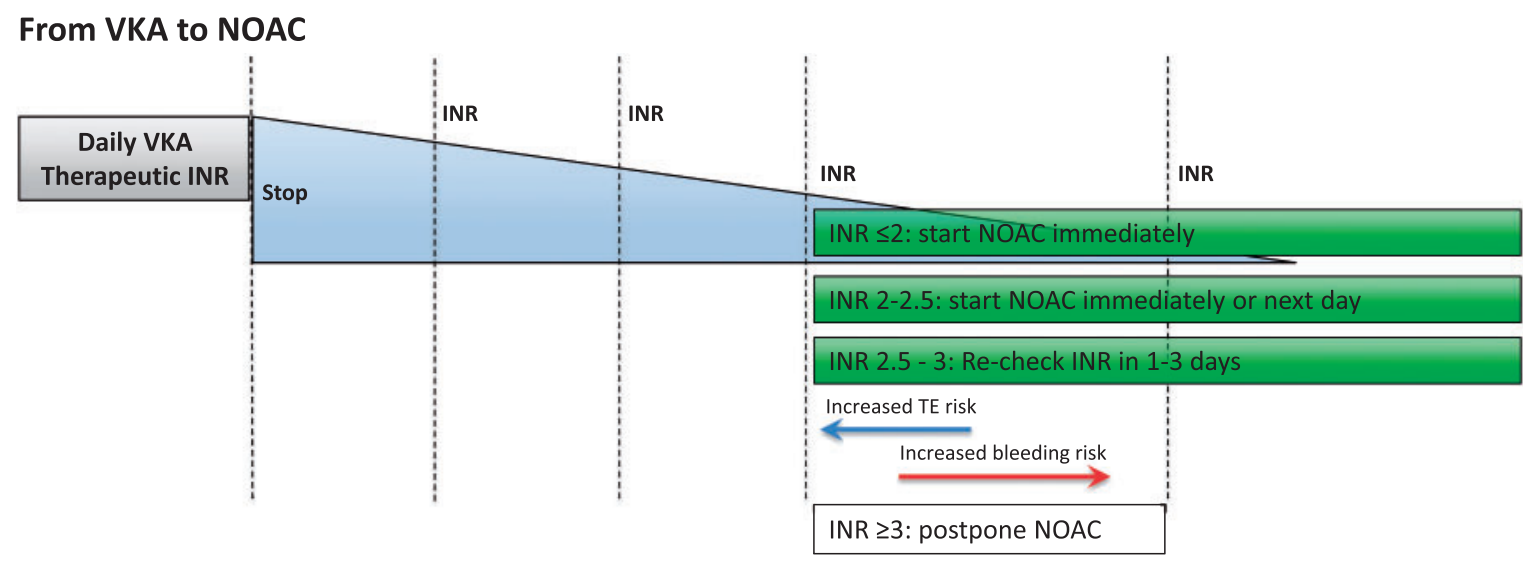

From NOAC to VKA

After 3-5 days: INR (sampled before NOAC intake)

\begin{tabular}{|l|l|l|l|l|l|}
\hline Daily NOAC & $\begin{array}{l}\text { Continue NOAC } \\
\text { (half dose for edoxaban) }\end{array}$ \\
\hline & Start VKA (loading dose usually used for phenprocoumon) \\
\hline & (half dose for edoxaban)
\end{tabular}

Figure 2 Switching between vitamin $\mathrm{K}$ antagonists and non-vitamin $\mathrm{K}$ antagonist oral anticoagulants and vice versa. TE, thromboembolic.

has proven to minimize the risks of stroke and bleeding ${ }^{125}$ while, conversely, inadequate transitioning was associated with increased stroke rates. $^{126,127}$ Whether the half-dose bridging regimen also applies to transitioning of NOACs other than edoxaban is unknown.

When concomitant administration of a NOAC during the initiation of the VKA is not deemed appropriate, initiation of the VKA can be performed after switching the NOAC to LMWH (see below), which may be considered especially in patients with a high thromboembolic risk.

\section{Non-vitamin K antagonist oral}

\section{anticoagulant to parenteral anticoagulants} The parenteral anticoagulant [unfractionated heparin (UFH) and $\mathrm{LMWH}$ ] can be initiated when the next dose of the NOAC would be due.

\section{Parenteral anticoagulant to non-vitamin $\mathrm{K}$ antagonist oral anticoagulant}

Intravenous UFH: NOACs can usually be started 2 (to 4) h after intravenous UFH (half-life $2 \mathrm{~h}$ ) is discontinued.

Low molecular weight heparin: NOACs can be initiated when the next dose of LMWH would be due. Care should be taken in patients with renal impairment where the elimination of $\mathrm{LMWH}$ may be prolonged.

\section{Non-vitamin K antagonist oral anticoagulant to non-vitamin $\mathrm{K}$ antagonist oral anticoagulant}

The alternative NOAC can be initiated when the next dose of the initial NOAC is due, except in situations where higher than therapeutic plasma concentrations are expected (e.g. in a patient with impaired renal function). In such situations, a longer interval in between NOACs is recommended.

\section{Aspirin or clopidogrel to non-vitamin K antagonist oral anticoagulant}

The NOAC can be started immediately and aspirin or clopidogrel stopped, unless combination therapy is deemed necessary (see chapter 14).

\section{Pharmacokinetics and drug- drug interactions of non-vitamin $K$ antagonist oral anticoagulants}

Treatment with VKAs requires careful consideration of multiple food and drug-drug interactions. Despite fewer interactions with the 
NOAC drugs, physicians should consider the pharmacokinetic interactions of accompanying drugs and comorbidities when prescribing NOACs. This section aims to provide a simple guide to deal with such situations. However, every patient may require more specific consideration, especially when a combination of interfering factors is present. Knowledge regarding interactions (with effect on plasma levels and/or on clinical effects of NOAC drugs) is expanding, so that new information may modify existing recommendations.

The absorption, distribution, metabolism, and excretion of the different NOACs are summarized in the previous version of the guide. ${ }^{2}$ An important interaction mechanism for all NOACs consists of significant gastrointestinal re-secretion over a P-glycoprotein (P-gP) transporter after absorption in the gut. Competitive inhibition of this pathway will result in increased plasma levels. The P-gp transporter is also involved in renal clearance. ${ }^{128}$ Many drugs used in AF patients are $\mathrm{P}$-gP inhibitors (e.g. verapamil, dronedarone, amiodarone, and quinidine). CYP3A4-type cytochrome P450-dependent elimination is relevantly involved in the hepatic clearance of rivaroxaban and apixaban. $^{129}$ Strong CYP3A4 inhibition or induction may affect plasma concentrations, and should be evaluated in context (see Tables 3-5 and colour coding, discussed below). Non-metabolic clearance of apixaban is diverse (including excretion of the unchanged compound by $>50 \%$ ), which reduces the potential for drug-drug interaction. ${ }^{130}$ In general, NOAC use is not recommended in combination with drugs that are strong inhibitors of both CYP3A4 and P-gp. Conversely, strong inducers of P-gP and/or CYP3A4 (such as rifampicin, carbamazepine, etc.) will markedly reduce NOAC plasma levels; such combinations should be avoided or used with great caution and surveillance.

Specific dosing algorithms for the different NOACs have been evaluated in large Phase III clinical trials and resulted in documented efficacy and safety of the respective agent. Of note, only one Phase III study prospectively used concomitant therapy with certain drugs as a dose reduction criterion (dose reduction of edoxaban in ENGAGE$A F$ in patients treated with potent $\mathrm{P}$-gp inhibitors verapamil, quinidine, or dronedarone). Dose reduction of all NOACs is primarily recommended along the published dose reduction criteria (see chapter 15). Whenever possible, the tested standard doses of NOACs should be used.

However, there is some rationale for reducing the dose of NOACs in patients with a high bleeding risk and/or when a higher plasma level of the drug can be anticipated based on a combination of factors. ${ }^{3,151-154}$ Prospective clinical trial data only exist for "lower doses' of dabigatran (110 mg BID) and edoxaban (30/15 mg OD; but not approved). For dabigatran $110 \mathrm{mg}$ BID, a similar stroke risk and reduced major bleeding vs. warfarin was observed ${ }^{28}$; however, this was in an unselected AF population and not in selected high-risk patients in whom plasma levels may be increased and the benefit of a reduction in major bleeding may be lost. ${ }^{152,155}$ For edoxaban 30/ $15 \mathrm{mg}$ OD a 41\% higher ischaemic stroke risk compared with a wellcontrolled warfarin arm (median TTR >68\%) was observed leading to non-approval of this dosing regimen; at the same time, a reduction in major bleeding, cardiovascular- and all-cause mortality was observed compared with warfarin. ${ }^{31,153}$ These data represent the only available RCT evidence of a 'lower dose' of a NOAC for stroke prevention in AF on hard clinical endpoints. ${ }^{28,31}$ In contrast, no 'lower dose' arm was included in ROCKET-AF (for rivaroxaban) or
ARISTOTLE (for apixaban) and as such, no clinical outcome data are available for the use of these doses outside the tested dose reduction algorithms. (Of note, a small study in Japanese patients investigated the use of $15 \mathrm{mg}$ rivaroxaban as standard dose for stroke prevention in Japanese patients with apparently preserved efficacy, but the implications of these results outside this setting are unclear. ${ }^{156}$

The use of plasma level monitoring for NOAC dose-adjustment or in the setting of 'off label' lower dose prescription (see chapters 7 and 8) is discouraged for the vast majority of patients due to the lack of outcome data to support such an approach. Indeed, an increased risk of bleeding frequently goes along with an increased risk of stroke due to the overlapping risk factors (including advanced age, frailty etc.), and inappropriate use of a reduced dose may result in lack of stroke prevention. ${ }^{157}$ However, in rare cases of potentially substantial drug-drug interactions or special situations in which a certain NOAC is preferred for certain reasons (e.g. patients after transplantation, patients on HIV medication etc.) this may be considered (Figure 3). Importantly, this approach should be limited to centres with extensive experience in the performance and interpretation of such assays as well as in the care of NOAC-treated patients.

In summary, possible drug-drug interactions, especially when combined with other clinical risk factors affecting NOAC plasma levels are important aspects for choosing a specific NOAC for a specific patient. Table 3 gives an overview of the effect of various frequently used agents on NOAC plasma levels; Table 4 focusses on common cancer drugs (see also chapter 19), Table 5 on antiepileptic drugs (see also chapter 18.4). Taking into consideration these factors as well as the setup and results from the large randomized NOAC outcome trials the algorithm shown in Figure 3 may assist in a rational selection of a specific NOAC and/or a 'reduced dose' based on drug-drug interactions and other clinical risk factors. Unfortunately, for many potential interactions with drugs that are often used in AF patients no detailed information is available yet (hatched in the tables).

\section{Food intake, antacids, and nasogastric tube administration}

Rivaroxaban $15 \mathrm{mg} / 20 \mathrm{mg}$ for stroke prevention in $\mathrm{AF}$ needs to be taken with food [the area under the curve (AUC) plasma concentrations increases by $39 \%$ to a very high bioavailability of almost $100 \%$ ], while there is no food interaction with the other NOACs. The concomitant use of PPIs and $\mathrm{H} 2$-blockers leads to a small reduction in bioavailability of dabigatran, but without effect on clinical efficacy. ${ }^{158,159}$ There is also no relevant antacid interaction for the other NOACs. ${ }^{140,160,161}$ There are no pharmacokinetic data on fish oil supplements for any of the NOACs, but interaction is unlikely.

Data have shown that administration in crushed form, e.g. via a nasogastric tube, does not alter the bioavailability for apixaban, rivaroxaban, and edoxaban. ${ }^{162-164}$ Also an oral solution of apixaban $5 \mathrm{mg}$ (12.5 mL of $0.4 \mathrm{mg} / \mathrm{mL}$ oral solution administered via oral syringe together with $240 \mathrm{~mL}$ of water) has been developed, which has shown comparable exposure as the tablet formulation. ${ }^{165}$ In contrast, dabigatran capsules must not be opened as it results in a substantial increase in drug bioavailability (+75\% per SmPC). 
Table 3 Effect of drug-drug interactions and clinical factors on NOAC plasma levels ('area under the curve')

\begin{tabular}{|c|c|c|c|c|c|}
\hline & Via & $\begin{array}{l}\text { Dabigatran } \\
\text { etexilate }\end{array}$ & Apixaban & Edoxaban & Rivaroxaban \\
\hline P-gp substrate & & Yes & Yes & Yes & Yes \\
\hline CYP3A4 substrate & & No & Yes $(\approx 25 \%)$ & No $(<4 \%)$ & Yes $(\approx 18 \%)^{131}$ \\
\hline \multicolumn{6}{|c|}{ Antiarrhythmic drugs } \\
\hline Amiodarone & moderate $\mathrm{P}$-gp competition & +12 to $60 \%$ SmPC & No PK data ${ }^{a}$ & $+40 \%^{132-134}$ & Minor effect ${ }^{a}$ \\
\hline Digoxin & P-gp competition & No effect ${ }^{S m P C}$ & No effect ${ }^{135}$ & No effect & No effect ${ }^{\text {SmPC }}$ \\
\hline Diltiazem & $\begin{array}{l}\text { P-gp competition and weak } \\
\text { CYP3A4 inhibition }\end{array}$ & No effect ${ }^{S m P C}$ & $+40 \%^{136}$ & & No effect \\
\hline Dronedarone & $\begin{array}{l}\text { P-gP competition and } \\
\text { CYP3A4 inhibition }\end{array}$ & $\begin{array}{l}+70 \text { to } 100 \% \\
\text { (US: } 2 \times 75 \mathrm{mg} \text { if } \\
\mathrm{CrCl} 30-50 \mathrm{~mL} / \mathrm{min} \text { ) }\end{array}$ & & $+85 \%$ & $\begin{array}{l}\text { Moderate effect, } \\
\text { should be avoided }\end{array}$ \\
\hline Quinidine & P-gp competition & $+53 \%{ }^{\mathrm{SMPC}}$ & & $\begin{array}{l}+77 \%^{137} \text { (no } \\
\text { dose reduction }\end{array}$ & $\begin{array}{l}\text { Extent of } \\
\text { unknown }\end{array}$ \\
\hline Verapamil & $\begin{array}{l}\text { P-gP competition (and } \\
\text { weak CYP3A4 inhibition) }\end{array}$ & $\begin{array}{l}+12 \text { to } 180 \% \text { SmPC } \\
\text { (if taken } \\
\text { simultaneously) }\end{array}$ & & $\begin{array}{l}+53 \% \\
(\mathrm{SR})^{137,142} \text { (no } \\
\text { dose reduction }\end{array}$ & No effect \\
\hline
\end{tabular}

\begin{tabular}{|c|c|c|c|c|c|}
\hline Atorvastatin & $\begin{array}{l}\text { P-gp competition and } \\
\text { CYP3A4 inhibition }\end{array}$ & $\begin{array}{l}\text { No relevant } \\
\text { interaction }\end{array}$ & & No effect & No effect \\
\hline Ticagrelor & P-gp competition & $\begin{array}{l}+25 \%^{\mathrm{SmPC}} \text { (give } \\
\text { loading dose } 2 \mathrm{~h} \\
\text { after dabigatran) }^{\mathrm{d}}\end{array}$ & & & \\
\hline \multicolumn{6}{|l|}{ Antibiotics } \\
\hline $\begin{array}{l}\text { Clarithromycin; } \\
\text { Erythromycin }\end{array}$ & $\begin{array}{l}\text { Moderate P-gP competition } \\
\text { and strong CYP3A4 } \\
\text { inhibition }\end{array}$ & +15 to $20 \%$ & $\begin{array}{l}+60 \% \text { AUC } \\
+30 \% C_{\max }\end{array}$ & $+90 \%^{\mathrm{SmPC}}$ & $\begin{array}{l}+34 \% \text { (Erythromy- } \\
\text { cin)/ }+54 \% \\
\text { (Clarithromycin) } \\
\text { SmPC129 }\end{array}$ \\
\hline Rifampicin & $\begin{array}{l}\text { P-gP/BCRP and CYP3A4/ } \\
\text { CYP2J2 inducers }\end{array}$ & Minus $66 \%$ SmPC & Minus $54 \%^{138}$ & $\begin{array}{l}\text { Minus } 35 \% \text {, but } \\
\text { with compensa- } \\
\text { tory increase of } \\
\text { active } \\
\text { metabolites }\end{array}$ & Up to minus $50 \%^{\mathrm{SmPc}}$ \\
\hline \multicolumn{6}{|l|}{ Antiviral drugs } \\
\hline $\begin{array}{l}\text { HIV protease inhibitors } \\
\text { (e.g. ritonavir) }\end{array}$ & $\begin{array}{l}\text { P-gp and BCRP competition } \\
\text { or inducer; CYP3A4 } \\
\text { inhibition }\end{array}$ & & & & Up to $+153 \%^{129}$ \\
\hline
\end{tabular}




\begin{tabular}{|c|c|c|c|c|c|}
\hline \multicolumn{6}{|l|}{ Fungostatics } \\
\hline Fluconazole & $\begin{array}{l}\text { Moderate CYP3A4 } \\
\text { inhibition }\end{array}$ & & No dat. & No data yet & $\begin{array}{l}+42 \% \text { (if systemically } \\
\text { administered) }\end{array}$ \\
\hline $\begin{array}{l}\text { Itraconazole; Ketoconazole; } \\
\text { Voriconazole }\end{array}$ & $\begin{array}{l}\text { potent P-gP and BCRP } \\
\text { competition; CYP3A4 } \\
\text { inhibition }\end{array}$ & $\begin{array}{l}+140 \text { to } 150 \% \\
\text { (US: } 2 \times 75 \mathrm{mg} \text { if } \\
\mathrm{CrCl} 30-50 \mathrm{~mL} / \\
\mathrm{min})\end{array}$ & $+100 \%^{136}$ & $\begin{array}{l}+87 \text { to } 95 \%^{132} \\
\text { (reduce NOAC } \\
\text { dose by } 50 \% \text { ) }\end{array}$ & Up to $+160 \%{ }^{\mathrm{SmPc}}$ \\
\hline Posaconasole & $\begin{array}{l}\text { Mild to moderate P-gp } \\
\text { inhibition }\end{array}$ & SmPC & SmPC & & SmPC \\
\hline \multicolumn{6}{|l|}{ Others } \\
\hline Naproxen & $\begin{array}{l}\text { P-gp competition; pharma- } \\
\text { codynamically increased } \\
\text { bleeding time }\end{array}$ & & $+55 \%{ }^{139}$ & No effect & \\
\hline H2B; PPI; Al-mg-hydroxide & Gl absorption & Minus $12-30 \%$ & No effect & No effect ${ }^{\mathrm{SmPc}}$ & No effect ${ }^{140}$ \\
\hline St. John's wort & $\begin{array}{l}\text { P-gp/BCRP and CYP3A4/ } \\
\text { CYP2J2 inducers }\end{array}$ & & & & \\
\hline \multicolumn{6}{|l|}{ Other factors } \\
\hline Age $\geq 80$ years & $\begin{array}{l}\text { Potential for Increased } \\
\text { plasma levels }\end{array}$ & & $b$ & c & \\
\hline Age $\geq 75$ years & $\begin{array}{l}\text { Potential for Increased } \\
\text { plasma levels }\end{array}$ & & & c & \\
\hline Weight $\leq 60 \mathrm{~kg}$ & $\begin{array}{l}\text { Potential for Increased } \\
\text { plasma levels }\end{array}$ & & b & $b$ & \\
\hline Renal function & Increased plasma level & \multicolumn{4}{|l|}{ See Figure 4} \\
\hline $\begin{array}{l}\text { Other increased } \\
\text { bleeding risk }\end{array}$ & & \multicolumn{4}{|c|}{$\begin{array}{l}\text { - Concomitant antiplatelet drugs; NSAID; systemic steroid therapy; other } \\
\text { - } \text { anticoagulants } \\
\text { - } \text { Recent surgery on critical organ (brain; eye) } \\
\text { - Frailty/falls risk } \\
\text { - St.p bleeding or predisposition (anaemia, thrombocytopenia) }\end{array}$} \\
\hline
\end{tabular}

The hatched colour coding indicates no clinical or PK data available, and recommendations are based on the respective NOAC SmPC (where available) or expert opinion. White: No relevant drug-drug interaction anticipated.

Yellow: Consider dose adjustment or different NOAC if 2 or more 'yellow' factors are present (see Figure 3).

Orange: Consider dose adjustment or different NOAC (see Figure 3).

Red: contraindicated/not recommended.

Brown: Contraindicated due to reduced NOAC plasma levels.

Blue: The label for edoxaban mentions that co-administration is possible in these cases, despite a decreased plasma level, which are deemed not clinically relevant. Since not tested prospectively, however, such concomitant use should be used with caution, and avoided when possible.

BCRP, breast cancer resistance protein; NSAID, non-steroidal anti-inflammatory drugs; H2B, H2-blockers; PPI, proton pump inhibitors; P-gp, P-glycoprotein; GI, gastrointestinal.

${ }^{a}$ Based on in vitro investigations, comparing the $\mathrm{IC}_{50}$ for $\mathrm{P}$-gp inhibition to maximal plasma levels at therapeutic dose, and/or on interaction analysis of efficacy and safety endpoints in the Phase-3 clinical trials. ${ }^{29,30}$ No direct PK interaction data available.

${ }^{\mathrm{b}}$ Dose reduction based on published criteria (see Table 13, Figure 3).

'Age had no significant effect after adjusting for weight and renal function.

${ }^{\mathrm{d} D a t a}$ from Phase I study. Evidence from Re-DUAL PCI indicate safety in the (small) subgroup on dabigatran and ticagrelor. ${ }^{141}$ 
Table 4 Anticipated effects of common anticancer drugs on non-vitamin $\mathrm{K}$ antagonist oral anticoagulants plasma levels

\begin{tabular}{|l|l|l|l|l|l|}
\hline & Via $^{142}$ & $\begin{array}{l}\text { Dabigatran } \\
\text { etexilate }\end{array}$ & Apixaban & Edoxaban & Rivaroxaban \\
\hline P-gp substrate & & Yes & Yes & Yes & Yes \\
\hline CYP3A4 substrate & & No & Yes $(\approx 25 \%)$ & No $(<4 \%)$ & Yes $(\approx 18 \%)$ \\
\hline
\end{tabular}

Antimitotic agents

\begin{tabular}{|l|l|l}
\hline Paclitaxel & $\begin{array}{l}\text { Moderate CYP3A4 } \\
\text { induction; CYP3A4/P-gP } \\
\text { Competition }\end{array}$ \\
\hline Vinblastine & $\begin{array}{l}\text { Strong P-gP induction; } \\
\text { CYP3A4/P-gP } \\
\text { competition }\end{array}$ \\
\hline Docetaxel, & $\begin{array}{l}\text { Mild CYP3A4 induction; } \\
\text { CYP3A4/P-gP } \\
\text { Competition }\end{array}$ \\
\hline Vincristine & $\begin{array}{l}\text { Mild CYP3A4 induction; } \\
\text { CYP3A4/P-gP } \\
\text { competition }\end{array}$ \\
\hline
\end{tabular}

Antimetabolites

\begin{tabular}{|l|l|} 
Metotrexate & $\begin{array}{l}\text { P-gp competition; no } \\
\text { relevant interaction } \\
\text { anticipated }\end{array}$ \\
\hline $\begin{array}{l}\text { Pemetrexed, Purine } \\
\text { analogs, Pyrimidine } \\
\text { analogs }\end{array}$ & $\begin{array}{l}\text { No relevant interaction } \\
\text { anticipated }\end{array}$ \\
\hline
\end{tabular}

Topoisomerase inhibitors

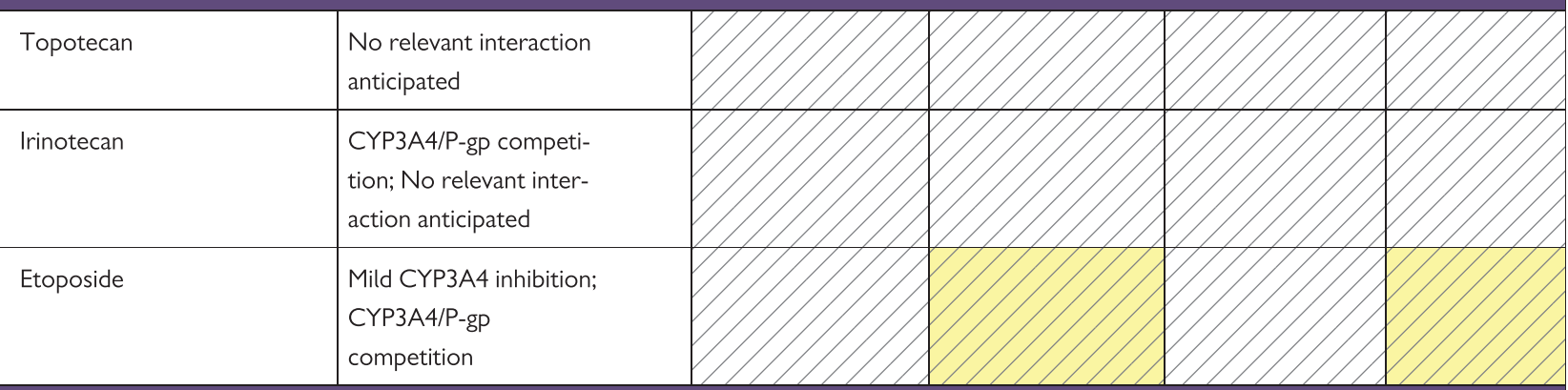

Anthracyclines/Anthracenediones

\begin{tabular}{|l|l|}
\hline Doxorubicin & $\begin{array}{l}\text { Strong P-gP induction, } \\
\text { mild CYP3A44 inhibition; } \\
\text { CYP3A4/P-gP } \\
\text { competition }\end{array}$ \\
\hline Idarubicin & $\begin{array}{l}\text { Mild CYP3A4 inhibition; } \\
\text { P-gP competition }\end{array}$
\end{tabular}




\begin{tabular}{|l|l|l}
\hline Daunorubicin & $\begin{array}{l}\text { P-gp competition; No } \\
\text { relevant interaction } \\
\text { anticipated }\end{array}$ \\
\hline Mitoxantrone & $\begin{array}{l}\text { No relevant interaction } \\
\text { anticipated }\end{array}$
\end{tabular}

\section{Alkylating agents}

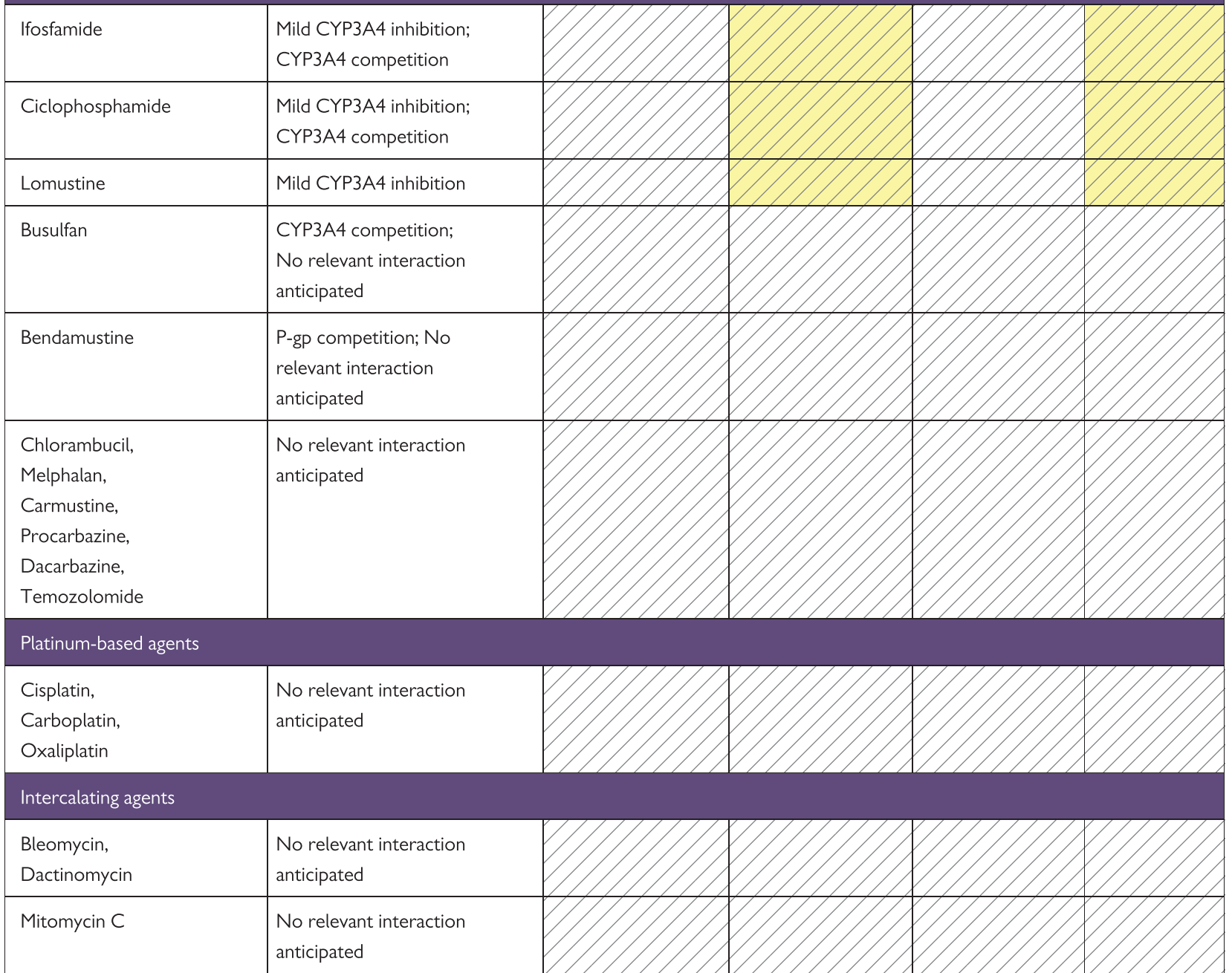

Tyrosine kinase inhibitors

\begin{tabular}{|l|l|}
\hline Imatinib, Crizotinib & $\begin{array}{l}\text { Strong P-gp inhibition, } \\
\text { moderate CYP3A4 } \\
\text { inhibition; CYP3A4/P-gP } \\
\text { competition }\end{array}$ \\
\hline Nilotinib, Lapatinib & $\begin{array}{l}\text { Moderate-to-strong P- } \\
\text { gp inhibition, mild } \\
\text { CYP3A4 inhibition; } \\
\text { CYP3A4/P-gP } \\
\text { competition }\end{array}$ \\
\hline Vemurafenib & $\begin{array}{l}\text { Moderate CYP3A4 } \\
\text { induction; CYP3A4/P-gP } \\
\text { competition }\end{array}$ \\
\hline
\end{tabular}




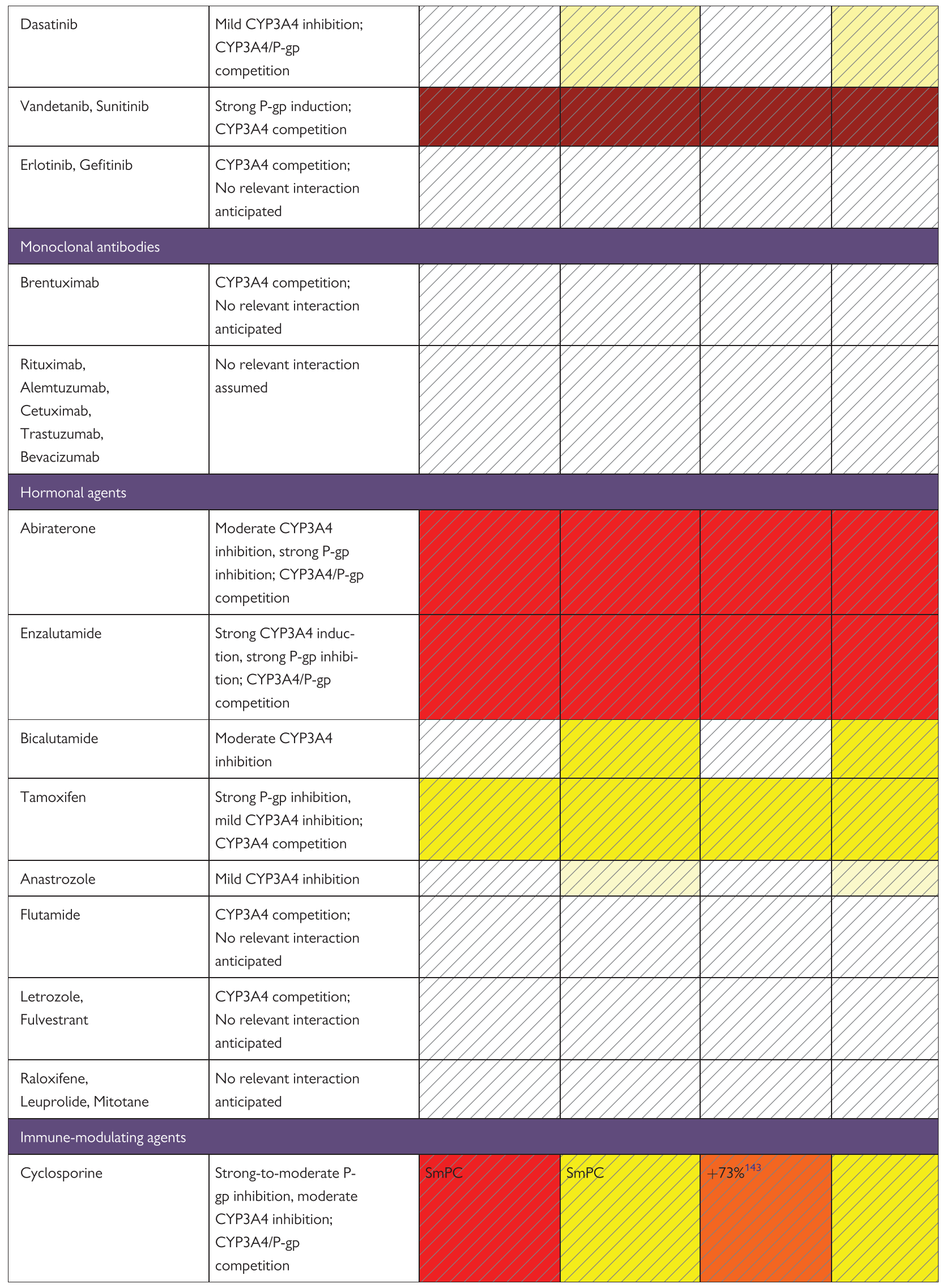




\begin{tabular}{|c|c|}
\hline Dexamethasone & $\begin{array}{l}\text { Strong CYP3A4/P-gp } \\
\text { induction; CYP3A4/P-gP } \\
\text { competition }\end{array}$ \\
\hline Tacrolimus & $\begin{array}{l}\text { Strong-to-moderate P- } \\
\text { gP inhibition, mild } \\
\text { CYP3A4 inhibition; } \\
\text { CYP3A4/P-gP } \\
\text { competition }\end{array}$ \\
\hline Prednisone & $\begin{array}{l}\text { Moderate CYP3A4 } \\
\text { induction; CYP3A4 } \\
\text { competition }\end{array}$ \\
\hline $\begin{array}{l}\text { Temsirolimus, } \\
\text { Sirolimus }\end{array}$ & $\begin{array}{l}\text { Mild CYP3A4 inhibition; } \\
\text { CYP3A4/P-gP } \\
\text { competition }\end{array}$ \\
\hline Everolimus & $\begin{array}{l}\text { CYP3A4 competition; } \\
\text { No relevant interaction } \\
\text { anticipated }\end{array}$ \\
\hline
\end{tabular}

Purine analogs: Mercaptopurine, Thioguanine, Pentostatin, Cladribine, Clofarabine, Fludarabine.

Pyrimidine analogs: Fluorouracil, Capeticabine, Cytarabine, Gemcitabine, Azacitadine, Decitabine.

Anticipated effects of common anticancer drugs on NOACs plasma levels. ${ }^{144}$

The hatched colour coding indicates no clinical or PK data available, and recommendations are based on the respective NOAC SmPC (where available) or expert opinion. Some of the colour codes will likely require adaptation as more data become available over time.

White: No relevant drug-drug interaction anticipated.

Yellow (light): Caution is needed in case of polypharmacy or in the presence of $\geq 2$ bleeding risk factors.

Yellow: Consider dose adjustment or different NOAC if 2 or more 'yellow' factors are present (see Figure 3).

Orange: Consider dose adjustment or different NOAC (see Figure 3).

Red: contraindicated/not recommended.

Brown (dark): Contraindicated due to reduced NOAC plasma levels.

Brown (light): Use with caution or avoid. Either expert opinion or the NOAC label mentions that co-administration is possible despite a decreased plasma level, which is deemed not clinically relevant (nevertheless, since not tested prospectively, such concomitant use should be used with caution, and avoided when possible).

Where no data or SmPC instructions were available, expert opinion was based on the following principles:

- Strong CYP3A4 and/or P-gp inducer-should not be used (dark brown).

- Moderate CYP3A4 or P-gP inducer - use with caution or avoid (light brown).

- Strong CYP3A4 and/or inhibitor-should not be used (red).

- Moderate CYP3A4 or P-gp inhibitor - use with caution, consider dose reduction or different NOAC (orange).

- Mild CYP3A4 and/or P-gp inducers or inhibitors - caution is needed with polypharmacy or in the presence of $\geq 2$ bleeding risk factors (yellow).

\section{Rate and rhythm control drugs}

Possible interactions are listed in Table 3. The P-gp inhibiting effects of verapamil on dabigatran levels are dependent on the verapamil formulation: when an immediate release preparation is taken within $1 \mathrm{~h}$ prior to dabigatran intake, plasma levels of dabigatran may increase up to $180 \%$. Separating both drugs' intake $\geq 2 \mathrm{~h}$ removes the interaction (but is hard to guarantee in clinical practice). With a slow-release verapamil preparation, there may be a $60 \%$ increase in dabigatran concentration. Pharmacokinetic data from the RE-LY trial showed an average $23 \%$ increase in dabigatran levels in patients taking verapamil. ${ }^{166}$ It is advised to use the lower dose dabigatran (110 mg BID) when combining it with verapamil ('orange', Table 3).

A similar interaction had initially been noted for edoxaban. ${ }^{167}$ However, after analysis of Phase III data, this interaction was considered not to be clinically relevant and no dose reduction is recommended in the European label. However, caution might be warranted in combination with other factors ('yellow', Table 3). On a more general level, these findings underline the difference between changes in plasma levels and influence on hard clinical endpoints. There are no specific interaction pharmacokinetic data available for apixaban or rivaroxaban with verapamil. Diltiazem has a lower inhibitory potency of P-gp, resulting in non-relevant interactions, ${ }^{166}$ although there is a $40 \%$ increase in plasma concentrations of apixaban ('yellow'; Table 3). ${ }^{136}$

For edoxaban a $40 \%$ increase in AUC was observed in patients on amiodarone with normal renal function. ${ }^{132}$ Of note, there was a significant interaction for amiodarone on the efficacy of the low-dose edoxaban regimen in the Phase III trial, exemplifying the potential impact of changed plasma levels. ${ }^{133}$ Nevertheless, dose reduction is not recommended in case of concomitant administration.

There is a strong effect of dronedarone on dabigatran plasma levels, which constitutes a contraindication for concomitant use. The interaction potential is considered moderate for edoxaban ('orange'), and dronedarone intake was a dose reduction criterion in the ENGAGE-AF protocol. ${ }^{31}$ There are no interaction pharmacokinetic data available for rivaroxaban and apixaban but effects on their plasma levels can be anticipated based on P-gP and CYP3A4 interactions, calling for caution (i.e. 'yellow') or avoidance (for rivaroxaban). Interestingly, a recent analysis of NOAC plasma levels before surgical 
Table 5 Anticipated effects of common antiepileptic drugs on non-vitamin $\mathrm{K}$ antagonist oral anticoagulants plasma levels

\begin{tabular}{|c|c|c|c|c|c|}
\hline & $\operatorname{Via}^{142,145,146}$ & $\begin{array}{l}\text { Dabigatran } \\
\text { etexilate }\end{array}$ & Apixaban $^{130}$ & Edoxaban & Rivaroxaban \\
\hline P-gp substrate & & Yes & Yes & Yes & Yes \\
\hline CYP3A4 substrate & & No & Yes $(\approx 25 \%)$ & No $(<4 \%)$ & Yes $(\approx 18 \%)$ \\
\hline \multicolumn{6}{|l|}{ Drug } \\
\hline Carbamazepine & $\begin{array}{l}\text { Strong CYP3A4/P-gp induction; } \\
\text { CYP3A4 competition }\end{array}$ & & & & \\
\hline Ethosuximide & $\begin{array}{l}\text { CYP3A4 competition; No relevant } \\
\text { interaction known/assumed }\end{array}$ & & & & \\
\hline Gabapentin & No relevant interaction known/assumed & & & & \\
\hline Lamotrigine & $\begin{array}{l}\text { P-gp competition; No relevant interaction } \\
\text { known/assumed }\end{array}$ & & & & \\
\hline Levetiracetam & P-gp induction; P-gp competition & & & & \\
\hline Oxcarbazepine & CYP3A4 induction; P-gP competition & & & & \\
\hline Phenobarbital & Strong CYP3A4/P-gp induction; P-gp competition & & SmPC & SmPC & SmPC \\
\hline Phenytoin & Strong CYP3A4/P-gp induction; P-gp competition & SmPC, Ref. ${ }^{148}$ & & SMPC & SmPC \\
\hline Pregabalin & No relevant interaction known/assumed & & & & \\
\hline Topiramate & CYP3A4 induction; CYP3A4 competition & & & & \\
\hline Valproic acid & CYP3A4/P-gp induction & & & & Ref. ${ }^{1 / 2}$ \\
\hline Zonisamide & $\begin{array}{l}\text { CYP3A4 competition; No relevant } \\
\text { interaction known/assumed }\end{array}$ & & & & \\
\hline
\end{tabular}

Anticipated effects of common antiepileptic drugs on NOACs plasma levels. ${ }^{147,150}$

The hatched colour coding indicates no clinical or PK data available, and recommendations are based on the respective NOAC SmPC, where available, or expert opinion.

Some of the colour codes will likely require adaptation as more data become available over time.

White: No relevant drug-drug interaction anticipated.

Brown (dark): Contraindicated due to reduced NOAC plasma levels.

Brown (light): Use with caution or avoid - either the label for the respective NOAC mentions that co-administration is possible despite a decreased plasma level, which are deemed not clinically relevant (nevertheless, since not tested prospectively, such concomitant use should be used with caution, and avoided when possible) or expert opinion. Where no data or SmPC instructions were available, expert opinion was based on the following principles:

- Strong CYP3A4 and/or P-gp inducer-should not be used (dark brown).

- Moderate CYP3A4 or P-gp inducer-use with caution or avoid (light brown).

- Strong CYP3A4 and/or inhibitor-should not be used (red).

- Moderate CYP3A4 or P-gp inhibitor - use with caution, consider dose reduction or different NOAC (orange).

- Mild CYP3A4 and/or P-gP inducers or inhibitors - caution is needed with polypharmacy or in the presence of $\geq 2$ bleeding risk factors (yellow).

intervention demonstrated that concomitant intake of verapamil, dronedarone, or amiodarone was significantly associated with higher pre-operative plasma levels. ${ }^{168}$

\section{Other drugs}

Table 3 also lists the potential interaction mechanisms for other drugs and their possible clinical relevance. Since some drugs are inhibitors of both CYP3A4 and P-gP, they may have an effect on NOAC plasma levels although the P-gP and/or CYP3A4 effect in itself is less pronounced. In general, although NOACs are substrates of CYP enzymes or $\mathrm{P}$-gp/breast cancer resistance protein (BCRP), they do not inhibit or induce any of them.

Co-administration of NOACs with other substrates of CYP3A4 (e.g. midazolam), P-gp (e.g. digoxin), or both (e.g. atorvastatin) does not significantly alter plasma levels of these drugs.

The platelet inhibitor ticagrelor is a P-gp inhibitor. Concomitant administration of ticagrelor $180 \mathrm{mg}$ loading dose with dabigatran $110 \mathrm{mg}$ increased dabigatran $C_{\max }$ by $65 \%$ (AUC $+49 \%$ ), compared with dabigatran given alone. When a loading dose of $180 \mathrm{mg}$ ticagrelor was given $2 \mathrm{~h}$ after $110 \mathrm{mg}$ dabigatran etexilate, the increase of 


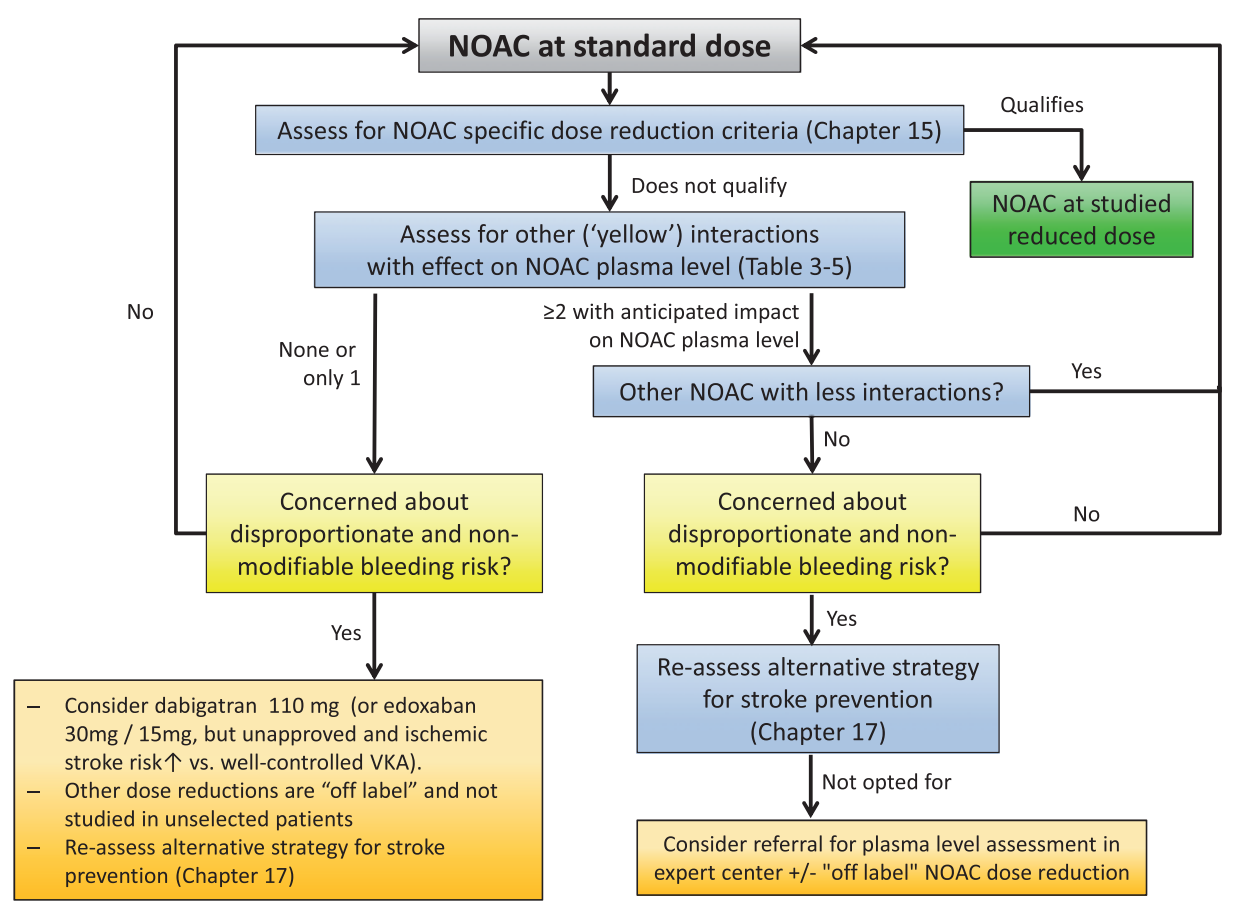

Figure $3 \mathrm{NOAC}$ selection based on drug-drug interactions and/or risk of bleeding. Use of plasma level measurements to guide dosing is generally discouraged and should only be used in rare cases of potentially substantial interactions or special situations, and only in centres with great experience in the performance and interpretation of such assays as well as the care of NOAC-treated patients.

dabigatran $C_{\max }$ and $\mathrm{AUC}$ was reduced to $+23 \%$ and $+27 \%$, respectively, compared with dabigatran given alone. As per the dabigatran SmPC, this staggered intake is the recommended administration strategy for starting with the loading dose of ticagrelor. Concomitant administration of $90 \mathrm{mg}$ ticagrelor BID (maintenance dose) with $110 \mathrm{mg}$ dabigatran increased the adjusted dabigatran $A \cup C$ and $C_{\max }$ by $26 \%$ and $29 \%$, respectively, compared with dabigatran given alone. These data are based on a Phase I study; the use of ticagrelor and dabigatran post-percutaneous coronary intervention ( $\mathrm{PCl}$ ) as studied in the RE-DUAL PCl study is discussed in detail later (see chapter 14). ${ }^{141}$

Of note, 'herbal' medicines are frequently underestimated regarding their potential for interaction, including the potent CYP3A4 and $\mathrm{P}$-gP inducer St. John's wort, although relevant interactions have been published (also outside the anticoagulation field). ${ }^{169}$ Due to the relevant decrease in NOAC levels, the concomitant use of St. John's wort is not recommended.

\section{Pharmacodynamic interactions}

Apart from the pharmacokinetic interactions, co-administration of NOACs with other anticoagulants, platelet inhibitors (e.g. aspirin, clopidogrel, ticlopidine, prasugrel, ticagrelor, others), and non-steroidal anti-inflammatory drugs increases the risk of bleeding. ${ }^{170-172}$ Therefore, such combinations should be carefully balanced against the potential benefit in each clinical situation. Coadministration of NOACs with dual antiplatelet drugs requires active measures to reduce time on triple therapy (see chapter 14).

\section{Polypharmacy}

Polypharmacy is a well-established risk factor for adverse events resulting from drug-drug interactions. ${ }^{173-175}$ In ROCKET-AF and ARISTOTLE, patients concomitantly taking several ( $\geq 5$ or $\geq 9$ ) medications experienced similar outcomes and consistent treatment effects of either NOAC relative to warfarin. ${ }^{174,175}$ Although reassuring, these findings are derived from post hoc analyses with many limitations. In addition, concomitant use of strong CYP3A4 inhibitors (e.g. ketoconazole, ritonavir) or inducers (e.g. phenytoin, rifampicin) was not allowed. Conversely, event rates with warfarin also increase in patients with polypharmacy, likely not only due to interactions but also due to the higher baseline risk of these patients. While polypharmacy in itself is not a contraindication for the use of NOACs, special care needs to be taken when treating these vulnerable patients (Tables 3-5; Figure 3).

\section{Non-vitamin $K$ antagonist oral anticoagulants in patients with chronic kidney disease or advanced liver disease}

Kidney and liver function both play an important role in the metabolism and elimination of NOACs. 


\section{Oral anticoagulation in chronic kidney disease}

There is a bidirectional interaction between AF and chronic kidney disease (CKD): AF facilitates the development or progression of $C K D$, and the prevalence and incidence of $A F$ increases with decreasing renal function. ${ }^{176-179}$ Patients with AF and CKD have an increased morbidity and mortality due to their excessive risk for both thromboembolic and severe bleeding events, making risk stratification and treatment challenging. ${ }^{180,181}$ In addition, all four NOACs are at least partly eliminated by the kidneys. Dabigatran has the greatest extent of renal elimination (80\%), whereas $50 \%, 35 \%$, and $27 \%$ of edoxaban, rivaroxaban, and apixaban, respectively, are cleared via the kidneys as unchanged drug (Table 6).

Clinical decisions on how to treat an AF patient with CKD who needs $O A C$ requires the assessment of renal function. Basic information on the diagnosis/staging of CKD and assessment of renal function is provided in Table 7. Several equations are available to gauge a patient's renal function, all with inherent strengths and limitations. The CKD-EPI equation estimating the glomerular filtration rate is recommended by the National Kidney Foundation because it has been shown to be reliable across the range of CKD stages. ${ }^{187}$ However, in the context of NOAC treatment, renal function should preferably be estimated by calculating the $\mathrm{CrCl}$ using the Cockcroft-Gault method, which was used in most NOAC trials and therefore also in this Practical Guide. Importantly, CKD can only be diagnosed and assessed in stable situations and must not be confused with acute renal failure. In the latter case, serum creatinine levels and calculated $\mathrm{CrCl}$ may indicate mildly reduced (or even normal) renal function when in reality it is severely impaired. In situations with acute renal failure, any NOAC therapy needs to be discontinued and parenteral anticoagulation initiated (after careful risk-benefit analysis).

In patients on NOACs, renal function needs to be monitored diligently, at least yearly, to detect changes in renal function and adapt the dose accordingly. If renal function is impaired (i.e. $\mathrm{CrCl} \leq 60 \mathrm{~mL}$ min), a more frequent evaluation is recommended (e.g. by dividing $\mathrm{CrCl}$ by 10 to obtain the minimum frequency of renal function testing in months; Table 2). In patients with additional risk factors (e.g. older age, frail, multiple co-morbidities etc.), it may be evaluated even more frequently, especially if on dabigatran. Intercurrent acute illness (like infections, acute heart failure, etc.) may transiently affect renal function and should also trigger re-evaluation; importantly, patients need to be alerted that in such situations they should seek contact with their healthcare provider. This guidance is also presented in the updated NOAC Card.

On the other side of the spectrum, a possibly decreased efficacy of edoxaban $60 \mathrm{mg}$ OD compared with warfarin was observed in patients with a $\mathrm{CrCl}$ of $>95 \mathrm{~mL} / \mathrm{min}^{31}{ }^{31}$ Interestingly, as a result of these findings, further post hoc analyses revealed a similar effect also for Rivaroxaban ${ }^{188}$ and Apixaban. ${ }^{189}$ In 2015 the FDA issued a warning about the use of edoxaban in individuals with such a highnormal $\mathrm{CrCl}$, and recommended the use of other oral anticoagulants in these patients. Also the EMA advised that 'edoxaban should only be used in patients with high $\mathrm{CrCl}$ after a careful evaluation of the individual thromboembolic and bleeding risk'. A post hoc analysis of the ENGAGE AF data showed that despite the trend towards a decrease in relative efficacy of edoxaban $60 \mathrm{mg}$
OD in the upper range of $\mathrm{CrCl}$ in an exploratory (not pre-defined) subgroup analysis, the safety and net clinical benefit of edoxaban compared with warfarin were consistent across the spectrum of renal function. ${ }^{190}$

\section{Oral anticoagulant therapy in patients with mild or moderate CKD ( $\mathrm{CrCl} \geq \mathbf{3 0} \mathrm{mL} / \mathrm{min})$}

The benefit of VKAs in terms of reduced stroke and mortality is well established in AF patients with mild to moderate CKD. ${ }^{191-194}$ Compared with warfarin, all four NOACs showed consistent efficacy and safety in patients with mild to moderate CKD compared with nonCKD patients in the respective Subgroup analyses of pivotal NOAC trials. $^{190,195-199}$ In addition, the ARISTOTLE trial data analysis suggests that the bleeding benefit with apixaban compared with warfarin becomes significantly more prominent at lower $\mathrm{CrCl}$ values, while the stroke reduction benefit is maintained. ${ }^{181,197}$ In contrast, the bleeding benefit of $110 \mathrm{mg}$ dabigatran over warfarin is lost in patients with $\mathrm{CrCl}<50 \mathrm{~mL} / \mathrm{min}$ while maintaining a similar stroke risk reduction compared with VKA. ${ }^{195}$

A post hoc analysis of the RE-LY trial data showed a significantly faster rate of decline in renal function during the trial in patients on warfarin (especially at lower TTRs) compared with those on dabiga$\operatorname{tran}^{200}$ suggesting that it may delay the decline in renal function compared with warfarin. Moreover, it has been suggested that warfarin use may be associated with increased vascular calcification and/or the development of acute warfarin-related nephropathy with or without clinically overt haematuria. ${ }^{201}$

Appropriate dosing is an essential issue to be addressed when using NOACs in patients with CKD (Figure 4). While rivaroxaban, apixaban, and edoxaban doses were reduced according to renal function in their respective randomized clinical trials (RCTs), patients in the RE-LY trial were randomized to dabigatran $150 \mathrm{mg}$ BID or $110 \mathrm{mg}$ BID without dose reduction for renal insufficiency. Per SmPc, a recommendation for the use of dabigatran $110 \mathrm{mg}$ BID is made in patients with $\mathrm{CrCl}<50 \mathrm{~mL} / \mathrm{min}$ at high risk of bleeding. With the availability of three FXa inhibitors with less pronounced renal clearance, the use of the latter may be preferred in this patient population. The use of NOAC doses inconsistent with drug labelling has been associated with worse outcome; for example, underdosing of apixaban in patients with normal or only mildly reduced renal function has been associated with less effectiveness (i.e. higher stroke rates) and no additional safety benefit in a large 'real-world' AF cohort. ${ }^{202}$

\section{Oral anticoagulant therapy in patients with a $\mathrm{CrCl}$ of $15-29 \mathrm{~mL} / \mathrm{min}$}

There are no RCT data on the use of NOACs for stroke prevention in AF patients with severe CKD or on renal replacement therapy (RRT) since all landmark NOACs trials essentially excluded patients with a $\mathrm{CrCl}$ of $<30 \mathrm{~mL} / \mathrm{min}$ (except for a few patients on apixaban with $\mathrm{CrCl} 25-30 \mathrm{~mL} / \mathrm{min}$ ). However, VKA have also never been prospectively assessed in a RCT in this patient population.

Rivaroxaban, apixaban, and edoxaban (but not dabigatran) are approved in Europe for the use in patients with severe CKD (Stage 4, i.e. a $\mathrm{CrCl}$ of $15-29 \mathrm{~mL} / \mathrm{min}$ ), with the reduced dose regimen (see chapter 15 and Figure 4). In view of the individual NOACs' pharmacokinetics, dose-reduction criteria and available evidence from RCTs, 
Table 6 Absorption and metabolism of the different NOACs

\begin{tabular}{|c|c|c|c|c|}
\hline & Dabigatran $^{158,182}$ & Apixaban ${ }^{183}$ & Edoxaban ${ }^{184}$ & Rivaroxaban ${ }^{185,186}$ \\
\hline Bioavailability & $3-7 \%$ & $50 \%$ & $62 \%$ & $\begin{array}{l}15 \mathrm{mg} / 20 \mathrm{mg}: 66 \% \text { without food, } \\
80-100 \% \text { with food }\end{array}$ \\
\hline Prodrug & Yes & No & No & No \\
\hline $\begin{array}{l}\text { Clearance non-renal/renal } \\
\text { of absorbed dose }\end{array}$ & $20 \% / 80 \%$ & $73 \% / 27 \%$ & $50 \% / 50 \%$ & $65 \% / 35 \%$ \\
\hline Plasma protein binding & $35 \%$ & $87 \%$ & $55 \%$ & $95 \%$ \\
\hline Dialysability & $\begin{array}{l}50-60 \% \\
\text { (in part dialysable) }\end{array}$ & $\begin{array}{l}14 \% \\
\text { (in part dialysable) }\end{array}$ & $\begin{array}{l}\text { n.a. } \\
\text { (in part dialysable) }\end{array}$ & $\begin{array}{l}\text { n.a. } \\
\text { (in part dialysable) }\end{array}$ \\
\hline $\begin{array}{l}\text { Liver metabolism: } \\
\text { CYP3A4 involved }\end{array}$ & No & $\begin{array}{l}\text { Yes [elimination, } \\
\text { moderate contribution } \\
(\approx 25 \%)^{\mathrm{a}} \text { ] }\end{array}$ & $\begin{array}{l}\text { Minimal }(<4 \% \text { of } \\
\text { elimination) }\end{array}$ & Yes (hepatic elimination $\approx 18 \%)^{131}$ \\
\hline Absorption with food & No effect & No effect & $\begin{array}{l}6-22 \% \text { more; minimal } \\
\text { effect on exposure }\end{array}$ & +39\% more (see above) \\
\hline Absorption with $\mathrm{H} 2 \mathrm{~B} / \mathrm{PPI}$ & $\begin{array}{l}-12 \% \text { to } 30 \% \text { (not } \\
\text { clinically relevant) }\end{array}$ & No effect & No effect & No effect \\
\hline Asian ethnicity & $+25 \%^{166}$ & No effect & No effect & No effect \\
\hline \multirow[t]{2}{*}{ Elimination half-life } & \multirow[t]{2}{*}{$12-17 \mathrm{~h}$} & \multirow[t]{2}{*}{$12 \mathrm{~h}$} & \multirow[t]{2}{*}{$10-14 \mathrm{~h}$} & 5-9h (young) \\
\hline & & & & 11-13 h (elderly) \\
\hline Other & Dyspepsia (5-10\%) & & & $\begin{array}{l}\text { Intake of } 15 \mathrm{mg} / 20 \mathrm{mg} \text { with } \\
\text { food mandatory }\end{array}$ \\
\hline
\end{tabular}

a Hepatic metabolism in total of $\approx 25 \%$, mostly via CYP3A4, with minor contributions of CYP1A2, 2J2, 2C8, 2C9, and 2 C19.

the use of either apixaban or edoxaban may be preferable in these patients. Apixaban is least renally cleared (27\%), and the dose is reduced by $50 \%$ in rather stringent conditions according to its dose reduction algorithm; furthermore the relative safety of apixaban vs. warfarin has been demonstrated to increase with decreasing renal function. ${ }^{197}$ Edoxaban is $50 \%$ renally cleared, but its dose reduction to $50 \%$ is applied more rapidly and was tested in a large subgroup. Rivaroxaban has an intermediate renal clearance (33\%), and its dose is reduced less (by 25\%) under similar conditions as edoxaban. In the US (but not in Europe), a low dose dabigatran $75 \mathrm{mg}$ BID regimen has been approved for patients with severe CKD (a CrCl of 15-29 mL $\mathrm{min}$ ), based on pharmacokinetic simulations. Further randomized trial data are urgently required for these difficult to treat patients.

\section{Oral anticoagulant therapy in patients with a $\mathrm{CrCl}$ of $\leq \mathbf{1 5} \mathrm{mL} / \mathrm{min}$ and on dialysis}

Numerous observational studies yielded conflicting results for VKA regarding efficacy without a clear consistent benefit of VKA in patients with severe renal dysfunction, ${ }^{192-194,203}$ Most studies confirmed a significantly lower incidence of stroke and embolism under warfarin, but also a markedly increased bleeding risk. ${ }^{192-194}$ The only registry that assessed the net benefit found no changes in overall-mortality for warfarin in dialysis-dependent patients. ${ }^{193}$ Of note, the use of warfarin in patients with end-stage renal failure may in some cases result in calciphylaxis, a painful and often lethal condition caused by calcification and occlusion of cutaneous arteries and arterioles. ${ }^{204-208}$

The efficacy and safety of NOACs in patients with end-stage renal dysfunction and on dialysis is unclear and subject to ongoing studies. Registry data have shown a higher incidence of hospitalization or death from bleeding in dialysis-dependent patients started on off-label dabigatran or rivaroxaban compared with VKA. ${ }^{209}$ In the US (but not in Europe) apixaban $5 \mathrm{mg}$ BID is currently approved in chronic, stable dialysis-dependent patients. However, plasma levels with apixaban $5 \mathrm{mg}$ BID were recently shown to be supra-therapeutic. ${ }^{210}$ Levels similar to those in patients with normal renal function on the respective NOACs were found for apixaban $2.5 \mathrm{mg}$ BID in a small number of patients on dialysis, ${ }^{210}$ for edoxaban $15 \mathrm{mg}$ OD (in Japanese patients with severe renal insufficiency $)^{211}$ and rivaroxaban $10 \mathrm{mg}$ OD in endstage renal disease patients. ${ }^{212}$ It needs to be kept in mind, however, that plasma levels are a surrogate endpoint. In the absence of hard endpoint studies (which are currently ongoing, e.g. NCT02942407, NCT02933697), the routine use of NOACs in patient with severe 
Table 7 Criteria for diagnosing chronic kidney disease; estimation of renal function and categories of renal dysfunction

\begin{tabular}{|c|c|c|c|}
\hline Decreased GFR ${ }^{a}$ & \multicolumn{3}{|c|}{ GFR $<60 \mathrm{~mL} / \mathrm{min} / 1.73 \mathrm{~m}^{2}$} \\
\hline Markers of kidney damage $(\geq 1)$ & \multicolumn{3}{|c|}{$\begin{array}{l}\text { - Excessive albuminuria (AER } \geq 30 \mathrm{mg} / 24 \mathrm{~h} ; A C R \geq 30 \mathrm{mg} / \mathrm{g} \text { or } \geq 3 \mathrm{mg} / \mathrm{mmol} \text { ) } \\
\text { - Urine sediment abnormalities } \\
\text { - Electrolyte or other abnormality caused by tubular disorders } \\
\text { - Abnormal histology } \\
\text { - Structural abnormalities detected by kidney imaging } \\
\text { - History of kidney transplantation }\end{array}$} \\
\hline GFR category & CKD stage & GFR $^{\mathrm{a}}$ & Descriptions \\
\hline G1 & 1 & $\geq 90$ & Normal or high \\
\hline G2 & 2 & $60-89$ & Mildly decreased \\
\hline G3a & \multirow{2}{*}{3} & $45-59$ & Mildly to moderately decreased \\
\hline G3b & & $30-44$ & Moderately to severely decreased \\
\hline G4 & 4 & $15-29$ & Severely decreased \\
\hline G5 & 5 & $<15$ & Kidney failure (requires renal replacement therapy - dialysis or kidney transplantation) \\
\hline
\end{tabular}

Estimation of renal function in NOAC patients best by Creatinine Clearance (Cockroft-Gault):

$$
\mathrm{CrCl}[\mathrm{mg} / \mathrm{dl}]=\frac{(140-\text { age }) \times \text { weight }(\text { in } \mathrm{kg}) \times[0.85 \text { if female }]}{72 \times \text { serum creatinine }(\text { in } \mathrm{mg} / \mathrm{dL})]}
$$

Online calculators available at (e.g.): www.kidney.org/professionals/kdoqi/gfr_calculator; www.nephron.com/cgi-bin/CGSI.cgi; www.mdcalc.com/creatinine-clearance-cockcroftgault-equation; https://reference.medscape.com/calculator/creatinine-clearance-cockcroft-gault.

Popular Apps are NephroCalc, MedMath, MedCalc, Calculate by QxMD, and Archimedes.

CKD, chronic kidney disease; GFR, glomerular filtration rate; $\mathrm{AER}$, albumin excretion rate; $\mathrm{ACR}$, albumin-to-creatinine ratio; $\mathrm{CrCl}$, creatinine clearance.

${ }^{\mathrm{a}} \mathrm{mL} / \mathrm{min} / 1.73 \mathrm{~m}^{2}$.

renal dysfunction $(\mathrm{CrCl}<15 \mathrm{~mL} / \mathrm{min})$ as well as in patients on dialysis is best avoided. In fact, given the lack of strong evidence also for VKA in this patient population, the decision to anticoagulate remains a very individualized one requiring a multidisciplinary approach considering and respecting patients' preferences. ${ }^{180,208,213}$

There are no data on the use of NOACs in AF patients after kidney transplantation. If NOACs are used in such patients, the dosing regimen should be selected according to the estimated renal function, and caution is needed with respect to possible drug-drug interactions between the NOAC and concomitant immunosuppressive therapies (see chapter $\mathbf{5}$ ).

\section{Non-vitamin K antagonist oral anticoagulants in liver disease}

Advanced liver disease is associated with increased bleeding risk, but is also a prothrombotic disorder. ${ }^{214}$ In addition, significant liver disease can profoundly affect hepatic clearance and drug metabolism, and altered functionality of the liver enzymes and transporters may alter drug response and facilitate drug-induced liver injury. ${ }^{215}$

The use of VKAs in patients with advanced liver disease and coagulopathy (Table 8 ) is challenging due to intrinsically elevated INR values and difficulties in selecting appropriate VKA dosing. ${ }^{216}$ Patients with significant active liver disease including cirrhosis, or those with persistent (as confirmed by repeated assessment $\geq 1$ week apart) elevation of the liver enzymes or bilirubin [e.g. alanine transaminase or aspartate transaminase $\geq 2(-3)$ times the upper limit of normal (ULN) or total bilirubin $\geq 1.5$ times the ULN] were excluded from the landmark NOAC trials in $A F^{28-31}$ Consequently, all four NOACs are contraindicated in patients with hepatic disease associated with coagulopathy and clinically relevant bleeding risk including Child-Turcotte-Pugh C cirrhosis (Table 8). Rivaroxaban should also not be used in AF patients with Child B liver cirrhosis due to a >twofold increase in drug exposure in these patients. ${ }^{217}$ Dabigatran, apixaban and edoxaban may be used with caution in patients with Child $B$ cirrhosis (Table 8). ${ }^{218,219}$ Initiation and follow-up at a specialised centre in a multidisciplinary team (including a hepatologist and a haematologist) is recommended.

Due to the withdrawalnon-approval of the direct thrombin inhibitor ximelagatran from the market in 2006 as a result of its hepatotoxic side effects, ${ }^{220}$ there had been some concern about the potential of NOACs to cause drug-induced liver injury. However, no signal for increased hepatotoxicity has been observed in any of the NOAC trials. ${ }^{221}$ In fact, the risk of liver injury may even be lower than with VKA. ${ }^{222-224}$ 


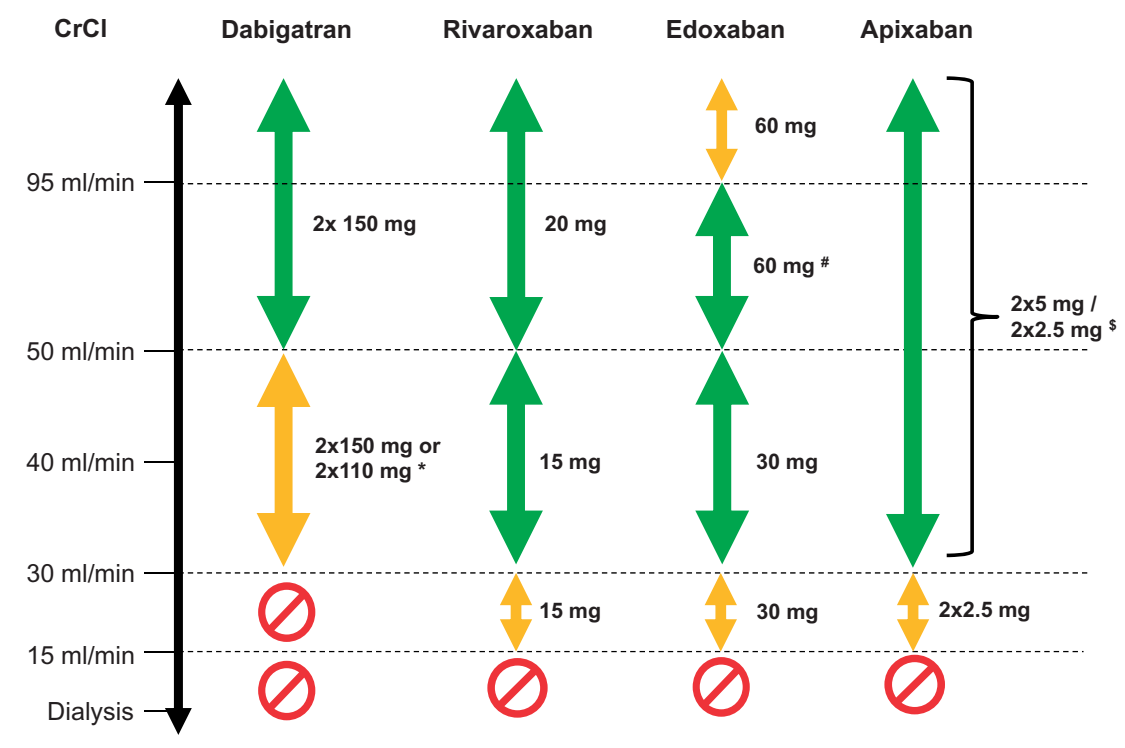

Figure 4 Use of non-vitamin $\mathrm{K}$ antagonist oral anticoagulants according to renal function. $* 2 \times 110 \mathrm{mg}$ in patients at high risk of bleeding (per $\mathrm{SmPc}$ ). ${ }^{\#}$ Other dose reduction criteria may apply (weight $\leq 60 \mathrm{~kg}$, concomitant potent P-Gp inhibitor therapy). ${ }^{\$} 2 \times 2.5 \mathrm{mg}$ only if at least two out of three fulfilled: age $\geq 80$ years, body weight $\leq 60 \mathrm{~kg}$, creatinine $\geq 1.5 \mathrm{mg} / \mathrm{dL}(133 \mu \mathrm{mol} / \mathrm{L})$. Orange arrows indicate cautionary use (dabigatran in moderate renal insufficiency, $\mathrm{FXa}$ inhibitors in severe renal insufficiency, edoxaban in 'supranormal' renal function); see text for details.

Table 8 Calculation of the Child-Turcotte-Pugh score and use of NOACs in hepatic insufficiency

\begin{tabular}{|c|c|c|c|c|}
\hline Parameters & 1 point & 2 points & \multicolumn{2}{|l|}{3 points } \\
\hline Encephalopathy & No & Grade 1-2 (suppressed with medication) & \multicolumn{2}{|c|}{ Grade 3-4 (refractory/chronic) } \\
\hline Ascites & No & Mild (diuretic-responsive) & \multicolumn{2}{|c|}{ Moderate-severe (diuretic-refractory) } \\
\hline Bilirubin & $<2 \mathrm{mg} / \mathrm{dL}$ & $2-3 \mathrm{mg} / \mathrm{dL}$ & \multicolumn{2}{|l|}{$>3 \mathrm{mg} / \mathrm{dL}$} \\
\hline & $<34 \mu \mathrm{mol} / \mathrm{L}$ & $34-50 \mu \mathrm{mol} / \mathrm{L}$ & \multicolumn{2}{|l|}{$>50 \mu \mathrm{mol} / \mathrm{L}$} \\
\hline Albumin & $>3.5 \mathrm{~g} / \mathrm{dL}$ & $2.8-3.5 \mathrm{~g} / \mathrm{dL}$ & \multicolumn{2}{|l|}{$<2.8 \mathrm{~g} / \mathrm{dL}$} \\
\hline & $>35 \mathrm{~g} / \mathrm{L}$ & $28-35 \mathrm{~g} / \mathrm{L}$ & \multicolumn{2}{|l|}{$<28 \mathrm{~g} / \mathrm{dL}$} \\
\hline INR & $<1.7$ & $1.71-2.30$ & \multicolumn{2}{|l|}{$>2.30$} \\
\hline Child-Pugh category & Dabigatran & Apixaban & Edoxaban & Rivaroxaban \\
\hline A (5-6 points) & No dose reduction & No dose reduction & No dose reduction & No dose reduction \\
\hline B (7-9 points) & Use with caution & Use cautiously & Use cautiously & Do not use \\
\hline C (10-15 points) & Do not use & Do not use & Do not use & Do not use \\
\hline
\end{tabular}




\section{How to measure the anticoagulant effect of non-vitamin $\mathrm{K}$ antagonist oral anticoagulants?}

Routine coagulation tests [prothrombin time (PT) and activated partial thromboplastin time (aPTT)] generally do not provide an accurate assessment of NOAC anticoagulant effects. In contrast, the latter can be measured via specific coagulation assays developed for the quantification of NOAC plasma levels. ${ }^{225-227}$ Most routine coagulometers are capable of measuring NOAC plasma levels within $\leq 30 \mathrm{~min}$. Institutions are recommended to consider 24/7 availability of these tests for emergency situations. In contrast, point-of-care tests are not yet available for patients on NOACs. ${ }^{228}$

Anti-FXa chromogenic assays are available to measure plasma concentrations of the FXa inhibitors using validated calibrators. Low and high plasma levels can be measured with acceptable inter-laboratory precision. The absence of anti-Xa activity with these assays excludes clinically relevant drug levels. Conversely, the diluted thrombin time (dTT) test as well as the ecarin chromogenic assay (ECA) display a direct linear relationship with dabigatran concentration and are suitable for the quantitative assessment of dabigatran concentrations.

The use of appropriate calibrators allows for the determination of plasma concentrations of all NOACs. Even though levels in clinical trials were measured using HPLC/MS, drug measurement and monitoring can be closely approximated using a calibrated ITT/ECA assay for dabigatran or chromogenic anti-FXa assay for FXa-inhibitors. It is recommended to primarily use plasma concentrations rather than anti-FXa activity or ITT to quantitatively assess the concentration of a NOAC. An overview of the expected peak and trough levels in patients on NOACs can be found in Table 9. When interpreting a coagulation assay in a patient treated with a NOAC, it is important to know when the NOAC was administered relative to the time of blood sampling. The maximum effect of the NOAC on the clotting test will occur at its maximal plasma concentration, which is approximately (1-)2-3 h after intake for each of these drugs (Table 9).

Of note, NOACs affect routine coagulation test (PT and aPTT), and also more specialized assays (such as lupus anticoagulant assays and coagulation factors) can be altered.

\section{Specific considerations Dabigatran}

For dabigatran, the aPTT may provide a qualitative assessment of dabigatran level and anticoagulant activity. The relationship between dabigatran and the aPTT is curvilinear. ${ }^{229}$ An aPTT in the normal range does not exclude dabigatran levels in the 'on therapy' range, but excludes drug levels above the 'on therapy' range when a sensitive assay is used.

Dabigatran has little effect on the PT and INR at clinically relevant plasma concentrations, which are therefore unsuitable for the assessment of the anticoagulant activity of dabigatran. ${ }^{228}$

The thrombin time $(T T)$ is very sensitive to the presence of dabigatran and a normal TT excludes even very low levels of dabigatran. The TT is not suited for the quantitative assessment of dabigatran plasma concentrations in the range expected with clinical use. In contrast, dTT tests and the ECA allow for the measurement of dabigatran levels in the range that is clinically relevant.

\section{Factor Xa inhibitors (rivaroxaban, apixaban, and edoxaban)}

The different factor Xa-inhibitors affect the PT and the aPTT to a varying extent. The aPTT cannot be used for any meaningful

Table 9 Plasma levels and coagulation assays in patients treated with non-vitamin $\mathrm{K}$ antagonist oral anticoagulants

\begin{tabular}{|c|c|c|c|c|}
\hline & Dabigatran 229,230 & Apixaban ${ }^{231}, \mathrm{SmPc}$ & Edoxaban ${ }^{184,232}$ & Rivaroxaban ${ }^{131,186}$ \\
\hline \multicolumn{5}{|c|}{ Expected plasma levels of NOACs in patients treated for AF (based on dTT/ECA for dabigatran and anti-FXa activity for Xa inhibitors) } \\
\hline $\begin{array}{l}\text { Expected range of plasma levels at peak } \\
\text { for standard dose }(\mathrm{ng} / \mathrm{mL})^{\mathrm{a}}\end{array}$ & $64-443$ & 69-321 & $91-321$ & $184-343$ \\
\hline $\begin{array}{l}\text { Expected range of plasma levels at trough } \\
\text { for standard dose }(\mathrm{ng} / \mathrm{mL})^{\mathrm{a}}\end{array}$ & $31-225$ & $34-230$ & $31-230$ & $12-137$ \\
\hline \multicolumn{5}{|c|}{ Expected impact of NOACs on routine coagulation tests } \\
\hline PT & $\uparrow$ & $(\uparrow)$ & $\uparrow(\uparrow)$ & $\uparrow \uparrow(\uparrow)$ \\
\hline aPTT & $\uparrow \uparrow(\uparrow)$ & $(\uparrow)$ & $\uparrow$ & $\uparrow$ \\
\hline ACT & $\uparrow(\uparrow)$ & $\uparrow$ & $\uparrow$ & $\uparrow$ \\
\hline TT & $\uparrow \uparrow \uparrow \uparrow$ & - & - & - \\
\hline
\end{tabular}

Ranges indicate the P5/95 percentiles for dabigatran, rivaroxaban, and apixaban, and the interquartile ranges for edoxaban.

The reagents influence the sensitivity of the PT for FXa inhibitors and of the aPTT for dabigatran. When a sensitive assay is used, normal aPTT excludes above on-therapy levels in dabigatran-treated patients, and normal PT excludes above on-therapy levels in rivaroxaban and edoxaban, but not apixaban treated patients. Point-of-care INR devices developed to monitor vitamin $\mathrm{K}$ antagonists do not accurately reflect the anticoagulant status of NOAC treated patients.

$\mathrm{ACT}$, activated clotting time; aPTT, activated partial thromboplastin time; dTT, diluted thrombin time; ECA, ecarin clotting assay; INR, international normalized ratio; PT, prothrombin time. 
evaluation of FXa inhibitory effect because of the limited prolongation, variability of assays, and paradoxical response at low concentrations. $^{233}$ Although Factor Xa-inhibitors demonstrate a concentration-dependent prolongation of the PT, the effect depends both on the assay and on the FXa inhibitor. Furthermore, PT is not specific and can be influenced by many other factors (e.g. hepatic impairment, vitamin K deficiency). ${ }^{233}$ For apixaban, the PT cannot be used for assessing the anticoagulant effect. For rivaroxaban and to a lesser extent edoxaban, the PT may provide some quantitative information, even though the sensitivity of the different PT reagents varies importantly and may be insensitive for the anti-FXa effect. ${ }^{226}$ Assessment of the sensitivity of the employed PT reagent for the $\mathrm{Xa}$ inhibitors is strongly recommended.

Importantly, conversion of PT to INR does not correct for the variation and even increases the variability. The INR (especially a point-of-care determined INR) is unreliable for the evaluation of FXa inhibitory activity. Furthermore, the prolongation of the PT/INR by NOACs can be misleading during the transition of a NOAC to a VKA. Therefore, switching needs to be executed diligently, as discussed in chapter 4.

\section{Impact of non-vitamin $K$ antagonist oral anticoagulants on other coagulation \\ assays}

NOACs also interfere with thrombophilia tests and the measurement of coagulation factors. Therefore, a time window of at least $24 \mathrm{~h}$ is recommended between the last intake of a NOAC and blood sampling to confidently assess coagulation parameters. This time window may be even longer for lupus anticoagulant measurements ( $\geq 48 h)$.

The activated clotting time (ACT) test is used as a point-of-care test in settings where high heparin doses are administered and where the aPTT is too sensitive (e.g. bypass surgery, coronary interventions, ablation procedures, etc.). It is a test on whole blood, based on contact activation. Dabigatran increases the ACT in a curvilinear fashion, consistent with the effects on aPTT. ${ }^{229}$ The ACT has not been investigated to gauge dabigatran anticoagulant activity in clinical practice. There is a small dose-dependent effect of apixaban, edoxaban, and rivaroxaban on the $A C T .{ }^{234,235}$ It seems reasonable to use the same target ACT levels for heparin titration in NOAC-treated patients undergoing interventions. However, since ACT is a non-standardized test, ACT target levels require centre validation. The ACT cannot be used to gauge FXa anticoagulant activity.

\section{Non-vitamin $K$ antagonist oral anticoagulant plasma level measurement: rare indications, precautions and potential pitfalls}

Non-vitamin K antagonist oral anticoagulants do not require monitoring of coagulation: neither the dose nor the dosing intervals need to be altered in response to changes in coagulation parameters for the currently registered indications. However, laboratory assessment of drug exposure and anticoagulant effect may help clinicians in emergencies as well as in special situations. Laboratory monitoring to guide long-term use can also be considered in exceptional patients with special characteristics. This, however, should only be done under the guidance of a coagulation expert and in the knowledge that hard clinical outcome data do not exist for such a strategy.

\section{Measurement in emergencies}

In emergencies such as bleeding (chapter 11), urgent procedures (chapter 13), or an acute stroke (chapter 17), routine coagulation tests are rapidly available and may quickly inform the clinician on recent exposure; specific assays may provide accurate assessment of plasma levels (chapter 7).

In case of serious bleeding, coagulation tests may help the clinician to support haemostasis (chapter 11). Coagulation tests may also uncover associated bleeding disorders. In case of urgent surgery as well as in exceptional cases of planned surgery with high-bleeding risk, coagulation tests may help the clinician define the timing of surgery (see chapters 12 and 13).

Information on drug exposure may also guide treatment in patients who present with acute thrombotic events, particularly in patients with acute ischaemic stroke for whom thrombolysis is considered (chapter 17). Other emergency situations where assessment of anticoagulant activity may be valuable include suspected overdosing or intoxication.

\section{Measurement before elective procedures}

In general, routine measurement of the anticoagulant activity is not recommended prior to elective procedures (chapter 12). When the timing since last intake is unknown or uncertain, or when there are concerns on the clearance of the drug because of special patient characteristics (potential drug-drug interactions, change in renal or hepatic function), it is reasonable to check the absence of clinically relevant plasma concentrations when specific assays are available. ${ }^{168}$ Importantly, however, there are currently no prospectively validated data with hard clinical endpoints on cut-off values of any coagulation test to guide the timing of elective or urgent surgery. ${ }^{236}$

\section{Monitoring during long-term exposure}

The expected drug levels while on therapy, as observed in clinical trials, are shown in Table 9. Importantly, no studies have investigated if measurement of drug levels and dose adjustment based on laboratory coagulation parameters reduces the risk for bleeding or thromboembolic complications, e.g. by dose reduction in case of higher than expected levels or by dose increase in case of lower than expected levels, during chronic treatment. As such, routine monitoring of plasma levels and subsequent dose adaptation is generally discouraged. For the (rare) patients with multiple factors that interfere with the pharmacokinetics of a given NOAC (e.g. the very obese; uncontrolled cancer patients receiving therapy for malignancies; treatment with anti-cancer drugs with unclear/unknown pharmacokinetic interactions), a reasonable strategy could be to verify that plasma levels are within the 'on treatment' range, taken into account the different 'on therapy' range for samples taken at peak or at trough levels (Table 9). However, this should only be performed in the hands of a coagulation expert with sufficient experience in the performance and interpretation of these assays as well as the care of these patients. 
Alternatively, reverting to VKA therapy in these very special situations may be an option.

\section{Over- and underweight patients}

Patients at the extremes of the weight spectrum (i.e. $<50 \mathrm{~kg}$ and $>120 \mathrm{~kg}$ ) have been underrepresented in the clinical trials, and NOAC use may be a challenge in these individuals (chapter 18). If NOAC treatment is decided on in such a patient, assessment of plasma trough levels may be considered.

\section{How to deal with dosing errors?}

Questions relating to dosing errors are very common in daily practice, and patients need to be informed on what to do in such cases. To avoid dosing errors as described below, patients on NOACs should be encouraged to make use of well-labelled weekly containers, with separate spaces for each dose timing. Importantly, however, dabigatran must not be taken out of its original bottle until immediately before intake. In order to provide a more uniform and simple practical advice some of the below recommendations do not fully align with all SmPCs. Also, patients' individual risk of stroke and bleeding need to be taken into consideration.

\section{Missed dose}

A forgotten dose may be taken until $50 \%$ of the dosing interval has passed. Hence, for NOACs with a BID dosing regimen (i.e. every $12 \mathrm{~h}$ ), a forgotten dose can be taken up until $6 \mathrm{~h}$ after the scheduled intake. For patients with a high stroke risk and low bleeding risk, this may be extended up until the next scheduled dose.

For NOACs with an OD dosing regimen, a forgotten dose can be taken up until $12 \mathrm{~h}$ after the scheduled intake. After this time point, the dose should be skipped and the next scheduled dose should be taken. The $12 \mathrm{~h}$ interval may be extended in patients with a high stroke risk.

\section{Double dose}

For NOACs with a BID dosing regimen, the next planned dose (i.e. after $12 \mathrm{~h}$ ) may be left out, with BID intake restarted $24 \mathrm{~h}$ after the double dose intake.

For NOACs with an OD dosing regimen, the patient should continue the normal dosing regimen, i.e. without skipping the next daily dose.

\section{Uncertainty about dose intake}

For NOACs with a BID dosing regimen, it is generally advisable to not take another tablet/capsule, but to simply continue with the regular dose regimen, i.e. starting with the next dose at the $12 \mathrm{~h}$ interval.

For NOACs with an OD dosing regimen, when thrombotic risk is high $\left(\mathrm{CHA}_{2} \mathrm{DS}_{2}\right.$-VASc $\left.\geq 3\right)$, it may generally be advisable to take another tablet and then continue the planned dose regimen. In case the thrombotic risk is low $\left(\mathrm{CHA}_{2} \mathrm{DS}_{2}-\mathrm{VASc} \leq 2\right)$, it is recommended to wait until the next scheduled dose.

\section{What to do if there is a (suspected) overdose without bleeding, or a clotting test is indicating a potential risk of bleeding?}

Excessive NOAC plasma concentrations potentially expose the patient to an increased risk of bleeding. This may occur when the patient has (intentionally) taken an overdose. Also intercurrent events such as acute renal failure (especially with dabigatran) or administration of drugs with known drug-drug interactions (see chapter 5) may increase NOAC plasma concentrations to supratherapeutic levels. In terms of management, it is important to distinguish between an overdose with bleeding complications (chapter 11) and without.

In case of a suspected overdose, coagulation tests can help to determine its degree and possible bleeding risk (see chapter 7). A normal aPTT excludes high levels of dabigatran; similarly a normal PT excludes very high levels of rivaroxaban and edoxaban. However, these routine coagulation tests are not appropriate for a quantitative assessment of high levels of these drugs.

Given the relatively short plasma half-life of the NOACs, a 'waitand-see' strategy can be used in most cases without active bleeding. The elimination half-life can be estimated taking into account age and renal function. As a result of limited absorption, a ceiling effect with little to no further increase in plasma exposure is seen at supratherapeutic doses of $\geq 50 \mathrm{mg}$ rivaroxaban. ${ }^{237}$ There are no data in this respect concerning the other $\mathrm{FXa}$ inhibitors or dabigatran.

In the case of recent acute ingestion of an overdose (especially when $\leq 2 \mathrm{~h}$ ago), the use of activated charcoal to reduce absorption may be considered for any NOAC (with a standard dosing scheme for adults of $30-50 \mathrm{~g}$ ) although clinical data on its effectiveness are lacking. ${ }^{238-240}$

If a more aggressive normalization of plasma levels is deemed necessary, or rapid normalization is not expected (e.g. major renal insufficiency) the steps outlined below (chapter 11) may need to be considered, including the use of a specific reversal agent. ${ }^{241}$ Only in exceptional cases, strategies to non-specifically support haemostasis awaiting clearance of the drugs may be considered, although clearly in these situations balancing the benefit of normalizing coagulation in a non-bleeding patient needs to be carefully weighed against a possibly strong prothrombotic effect.

\section{Management of bleeding under non-vitamin $\mathrm{K}$ antagonist oral anticoagulant therapy}

The Phase III NOAC studies have consistently shown that NOACs cause less intracranial and less life-threatening bleedings than warfarin, despite the absence of reversal strategies in these trials. Not only was there similar or even a reduced bleeding incidence, but patients experiencing a major (particularly extracranial) bleeding under NOACs were also shown to have a more favourable outcome than for bleeding under VKA treatment. ${ }^{240,242-245,378,379}$ Overall, a 
reduction in all-cause mortality was observed with NOACs vs. warfarin for stroke prevention in AF. ${ }^{246}$

Nevertheless, as more patients are being treated with NOACs, the absolute number of NOAC-related bleeding events will increase. Importantly, any bleeding is an opportunity to review the correct choice and dosing of the NOAC (see chapters 2, 5, 6, 15 and others) and to evaluate modifiable bleeding risk factors including suboptimally treated hypertension, labile INR (if on VKA) or erratic dosing, excessive alcohol intake and concomitant antiplatelet therapy, NSAIDs, glucocorticoids etc. (see also chapter 14). ${ }^{3}$

We recommend a hospital-wide policy concerning bleeding management under NOAC, developed in an interdisciplinary manner among cardiologists, haemostasis experts, emergency physicians/ intensivists and others. This protocol should describe the availability and indications of specific coagulation tests as well as of specific and nonspecific reversal agents. Such a policy needs to be communicated well and be easily accessible (e.g. on an Intranet site, in the emergency room, in pocket-sized leaflets etc.).

Strategies to manage bleeding complications in patients treated with NOACs rely on a precise analysis of the clinical situation.

(1) The type of bleeding: nuisance/minor, major non-life threatening, or life-threatening.

(2) The patient and his/her treatment: The exact time of last NOAC intake, prescribed dosing regimen, renal function, other factors influencing plasma concentrations (incl. co-medication, see also Table 3), and other factors influencing haemostasis (such as concomitant use of antiplatelet drugs).

Both routine coagulation tests and assays that specifically measure plasma levels of NOACs are important pillars in the assessment of NOAC related bleeding. Normal results of $\mathrm{dTT} /$ ecarin clotting time (for dabigatran) and anti-Xa activity (for anti-FXa treated patients) likely exclude relevant levels of the anticoagulant. Specific assays allow for the quantification of plasma levels of the anticoagulant (chapter 7). ${ }^{247}$ However, it needs to be kept in mind that restoration of coagulation does not necessarily result in improved clinical outcome. Conversely, conventional coagulation tests may be abnormal not only due to the effect of the NOAC itself, but for a variety of other reasons, particularly in the setting of severe bleeding.

Depending on the clinical scenario, the anticoagulant effects in a NOAC-treated patient who presents with bleeding can be addressed with the following strategies:

(1) Waiting until the anticoagulant activity of the NOAC effect wanes as a result of spontaneous clearance of the drug (Table 6), facilitated by maintaining (and potentially by stimulating) diuresis.

(2) Specific reversal: A specific reversal agent is available for dabigatran (idarucizumab, a humanized antibody fragment that specifically binds dabigatran). ${ }^{248}$ Specific agents for $\mathrm{FXa}$ inhibitors are undergoing clinical testing, including andexanet alfa (a recombinant human FXa analogue that competes with FXa to bind FXa inhibitors) ${ }^{249}$ and ciraparantag (PER 977), a small synthetic molecule that seems to have more generalized antagonistic effects. ${ }^{250}$

(3) Non-specific support of haemostasis using coagulation factors concentrates. There is increasing information about the effects of (activated) prothrombin complex concentrates in cohorts of NOACtreated patients with bleeding. ${ }^{251}$ In contrast, the use of fresh frozen plasma is not considered a useful reversal strategy, primarily due to the plasma abundance of NOACs which will inhibit newly administered coagulation factors upon activation and the resulting large volume that would need to be administered. ${ }^{247}$ Vitamin $\mathrm{K}$ and protamine administration have no role in the management of a bleeding under NOACs, but are useful in the management of bleeding under NOACs when vitamin K deficiency is suspected or in case of concomitant treatment with heparin, respectively.

\section{Nuisance and minor bleeding}

The clinical relevance of both nuisance and minor bleedings under NOAC therapy should not be underestimated as they are a frequent cause of treatment interruptions. Patients need to be made aware of the signs and symptoms of such bleedings and instructed to alert their healthcare provider in case of such an event (see chapter 2). Cessation or temporary interruption without consultation needs to be discouraged due to the subsequently increased thromboembolic risk.

Nuisance bleeds can usually be managed by delaying intake or withholding the NOAC for a maximum of one dose. Minor bleedings may require more aggressive therapy with a focus aimed at treating the cause of the bleeding (e.g. PPI for gastric ulcers, antibiotics for urinary tract infection, etc.). Epistaxis and gum bleeds can be treated with local anti-fibrinolytics.

In case of recurrent minor bleeding events without causal therapeutic options, an alternative NOAC with a potentially different bleeding profile should be considered while maintaining effective stroke prevention (see chapter $\mathbf{5}$ ).

A suspected or documented occult bleeding should trigger a work-up to uncover the underlying cause and the treatment thereof whenever possible.

\section{Non-life-threatening major bleeding}

Causal therapy to stop the bleeding and standard supportive measures (such as mechanical compression, endoscopic or surgical haemostasis, fluid replacement, transfusion, and other haemodynamic support) are the main pillars in the management of non-lifethreatening major bleeding. With increasing time a waning of the anticoagulant activity can be anticipated in view the relatively short elimination half-lives of all NOACs (see Tables 6, 10 and Figure 5). ${ }^{252}$

Adequate diuresis is recommended for all NOACs, but particularly in case of dabigatran (given the large degree of renal elimination of the drug). In addition, dialysis may be an option for non-life-threatening, severe bleeding with dabigatran in cases of severe renal failure if idarucizumab is not available. ${ }^{253,254}$ In contrast, dialysis has no significant impact in patients treated with any of the FXa inhibitors due to their high degree of protein plasma binding. ${ }^{255,256}$

The use of antifibrinolytics (e.g. tranexamic acid, $1 \mathrm{~g}$ i.v., repeated every $6 \mathrm{~h}$ if needed) or desmopressin $0.3 \mu \mathrm{g} / \mathrm{kg}$ i.v. infusion (with a maximal dosing of $20 \mu \mathrm{g}$ ) - especially in special situations with associated coagulopathy or thrombopathy - may be considered. Tranexamic acid has proven efficacy to support haemostasis, particularly in trauma-induced bleeding, with a favourable safety profile. $^{257,258}$ Even when not yet supported by clinical data its use can therefore be considered for bleeding under NOACs, especially in situations of severe bleeding where frequently many factors of the coagulation cascade are deficient. 
Table 10 Possible measures to take in case of bleeding

\begin{tabular}{|c|c|c|}
\hline & $\begin{array}{l}\text { Direct thrombin inhibitors } \\
\text { (dabigatran) }\end{array}$ & $\begin{array}{l}\text { FXa inhibitors } \\
\text { (apixaban, edoxaban, rivaroxaban) }\end{array}$ \\
\hline \multirow[t]{2}{*}{ Non life-threatening major bleeding } & \multicolumn{2}{|c|}{$\begin{array}{l}\text { - Inquire about last intake }+ \text { dosing regimen } \\
\text { - } \text { - } \text { Fluid replacement } \\
\text { - } \text { RBC substitution, if necessary } \\
\text { - } \text { Platelet substitution (in case of thrombocytopenia } \leq 60 \times 109 / \mathrm{L} \text { or thrombopathy) } \\
\text { - } \quad \text { Fresh frozen plasma not as reversal agent (may be considered as plasma expander) } \\
\text { - } \text { Tranexamic acid can be considered as adjuvant ( } 1 \mathrm{~g} \text { i.v., repeat every } 6 \mathrm{~h} \text {, if necessary) } \\
\text { - } \text { Desmopressin can be considered in special cases such as coagulopathy or thrombopathy; } \\
\quad 0.3 \mu \mathrm{g} / \mathrm{kg} \text { i.v. infusion (max dose } 20 \mu \mathrm{g} \text { ) }\end{array}$} \\
\hline & $\begin{array}{l}\text { - Estimate normalization of plasma levels: } \\
\text { - Normal renal function: } 12-24 \mathrm{~h} \\
\text { - } \mathrm{CrCl} 50-80 \mathrm{~mL} / \mathrm{min}: 24-36 \mathrm{~h} \\
\text { - } \mathrm{CrCl} 30-50 \mathrm{~mL} / \mathrm{min}: 36-48 \mathrm{~h} \\
\text { - } \mathrm{CrCl}<30 \mathrm{~mL} / \mathrm{min}: \geq 48 \mathrm{~h} \\
\text { - Maintain diuresis } \\
\text { - Consider idarucizumab (see below) }\end{array}$ & - Normalization of plasma levels: $12-24 \mathrm{~h}$ \\
\hline \multirow[t]{2}{*}{ Life-threatening bleeding } & $\begin{array}{l}\text { - All of the above } \\
\text { - Direct reversal: Idarucizumab } 5 \mathrm{~g} \text { i.v. in two } \\
\text { doses a } 2.5 \mathrm{~g} \text { i.v. no more than } 15 \mathrm{~min} \text { apart }\end{array}$ & $\begin{array}{l}\text { - All of the above } \\
\text { - Direct reversal: Andexanet alpha } \\
\text { (if available and approved) } \\
\text { - Bolus over } 15-30 \mathrm{~min} \text {, followed by } 2 \text {-h infusion } \\
\text { - Rivaroxaban (last intake }>7 \mathrm{~h} \text { before) or apixaban } \\
400 \mathrm{mg} \text { bolus, } 480 \mathrm{mg} \text { infusion @ } 4 \mathrm{mg} / \mathrm{min} \\
\text { - Rivaroxaban (last intake }<7 \mathrm{~h} \text { before or unknown) } \\
\text { or enoxaparin or edoxaban: } 800 \mathrm{mg} \text { bolus, } \\
960 \mathrm{mg} \text { infusion @ } 8 \mathrm{mg} / \mathrm{min}\end{array}$ \\
\hline & \multicolumn{2}{|c|}{$\begin{array}{l}\text { - Prothrombin complex concentrate (PCC) } 50 \mathrm{U} / \mathrm{kg} \text { (with additional } 25 \mathrm{U} / \mathrm{kg} \text { if clinically needed) } \\
\text { - Activated PCC } 50 \mathrm{U} / \mathrm{kg} ; \mathrm{max} 200 \mathrm{U} / \mathrm{kg} / \mathrm{day}) \text { : no strong data about additional benefit over PCC. } \\
\text { Can be considered before PCC, if available }\end{array}$} \\
\hline
\end{tabular}

$\mathrm{RBC}$, red blood cells; $\mathrm{CrCl}$, creatinine clearance; PCC, prothrombin complex concentrate.

${ }^{a}$ Andexanet alpha is currently neither approved nor available and final results of the ANNEXA-4 study are pending.

\section{Life-threatening bleeding}

Patients with life-threatening bleeding while treated with NOACs may benefit from its reversal in addition to the standard measures outlined above.

Importantly, even after direct reversal, significant NOAC concentrations may reappear in some patients and contribute to recurrent or continued bleeding (particularly after andexanet alpha, less after idarucizumab administration), ${ }^{249,259}$ underlining the necessity for continued clinical and laboratory monitoring.

\section{Idarucizumab}

In the REVERSE-AD study, idarucizumab was successfully used in patients on dabigatran presenting with major or life-threatening bleeding, or with the necessity of emergency surgery. Idarucizumab completely reversed the anticoagulant activity of dabigatran within minutes in almost all patients. ${ }^{248}$ It is hence recommended as firstline therapy in such situations. A total of $5 \mathrm{~g}$ idarucizumab is administered intravenously in two bolus doses of $2.5 \mathrm{~g}$ no more than $15 \mathrm{~min}$ apart (Figure 6). Continued clinical and laboratory monitoring is recommended, since a $5 \mathrm{~g}$ dose of idarucizumab may not completely neutralize an exceptional high level of dabigatran (e.g. in case of overdose or renal insufficiency). Also, low levels of dabigatran may reappear after 12-24h.

After $24 \mathrm{~h}$, dabigatran can be re-started if clinically indicated and feasible, with normal kinetics.

\section{Direct reversal of apixaban, edoxaban, or rivaroxaban (FXa-inhibitors)}

Based on the ongoing ANNEXA-4 study (which, in contrast to REVERSE-AD only includes patients with major/life-threatening 


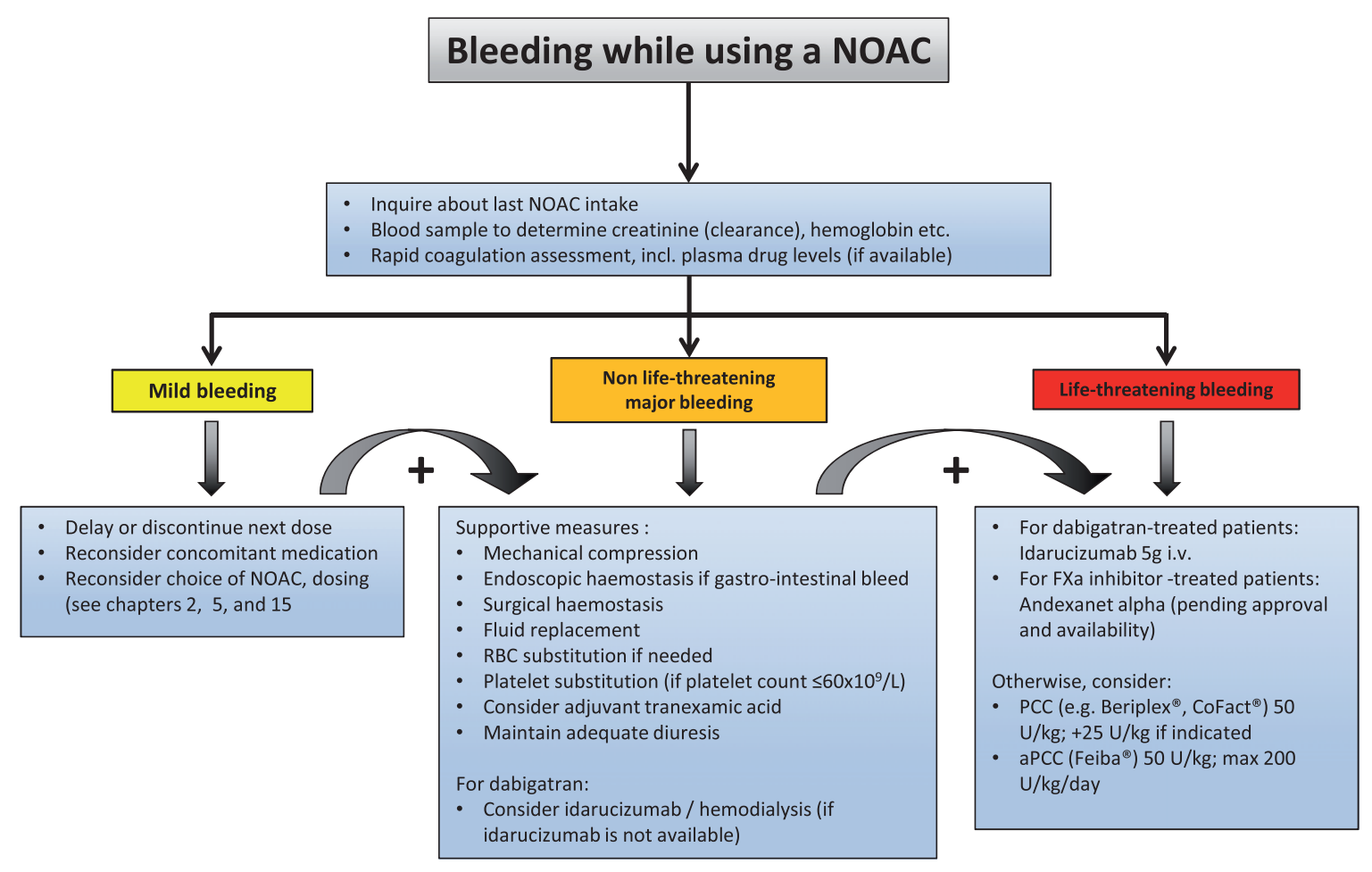

Figure 5 Management of bleeding in patients taking non-vitamin $\mathrm{K}$ antagonist oral anticoagulants.

bleeding), ${ }^{249}$ andexanet alpha may become the first choice of therapy in life-threatening bleeding under FXa-inhibitor therapy (pending its regulatory approval and availability). In the ANNEXA-4 study, the drug is administered as a bolus over $15-30$ min, followed by a $2-h$ infusion. The dosing depends on the NOAC and on the timing since last intake: For rivaroxaban (with the last intake $>7 \mathrm{~h}$ before reversal) or apixaban, a $400 \mathrm{mg}$ bolus is administered followed by a $480 \mathrm{mg}$ infusion $(4 \mathrm{mg} / \mathrm{min}$ ). For rivaroxaban (with the last intake $<7 \mathrm{~h}$ before reversal or unknown recent intake), edoxaban or enoxaparin, a $800 \mathrm{mg}$ bolus followed by a $960 \mathrm{mg}$ infusion $(8 \mathrm{mg} / \mathrm{min})$ is given (Figure 6). Importantly, reappearance of anticoagulant activity may occur after stopping the infusion. Therefore, it is currently less clear at what point in time and with which anticoagulant effect FXa inhibitors or heparin can be re-administered following andexanet alpha administration.

\section{Coagulation factors}

Clinical trials and registry data with NOACs have shown that administration of coagulation factors is rarely needed. ${ }^{251,260}$ Indeed, any NOAC-antagonizing effect has to be balanced carefully against the potential prothrombotic effect. Animal experiments as well as studies in healthy volunteers have indicated the potential usefulness of PCCs and activated PCCs (aPCC) for the normalization of coagulation parameters under NOAC treatment as a surrogate for haemostatic support. ${ }^{261-267}$ As indicated above, data from the large Phase III trials demonstrated that outcomes of bleedings under NOACs were similar (if not better) than in the VKA arm with similar treatment used (including PCC/aPCC). ${ }^{240,242-243}$ The efficacy on clinical outcomes of PCCs or aPCCs in patients taking NOACs who are actively bleeding has not been firmly established in a RCT. However, several observational studies in patients with major bleeding have been published (with some inherent limitations including the retrospective, noncontrolled setting as well as absence of a control group) indicating that (a)PCCs appeared to be efficacious in supporting haemostasis. $^{268,269}$

The administration of PCCs or aPCCs can be considered in a patient with life-threatening bleeding if immediate haemostatic support is required, especially in situations where a specific reversal agent is not available (Table 10). The choice between PCC and aPCC may depend on their availability and the experience of the treatment centre. Particularly aPCC induces a strong pro-coagulant effect and should only be used by physicians experienced in their use. PCC and aPCC are preferred over recombinant activated factor Vlla (NovoSeven, $90 \mu \mathrm{g} / \mathrm{kg}$ ) given the absence of any outcome data and the latter's pronounced pro-coagulant effect. ${ }^{247,270}$

\section{Anticoagulation post-extracranial bleeding}

In most cases of nuisance or minor bleeding anticoagulation can be re-started, sometimes simply by delaying or skipping a single dose. All other bleedings, particularly life-threatening bleeding episodes, require a careful re-assessment of the risks and benefits of reinitiating anticoagulation. In most cases of bleedings due to secondary (e.g. bleeding post-trauma) or reversible causes (e.g. genito-urinary 


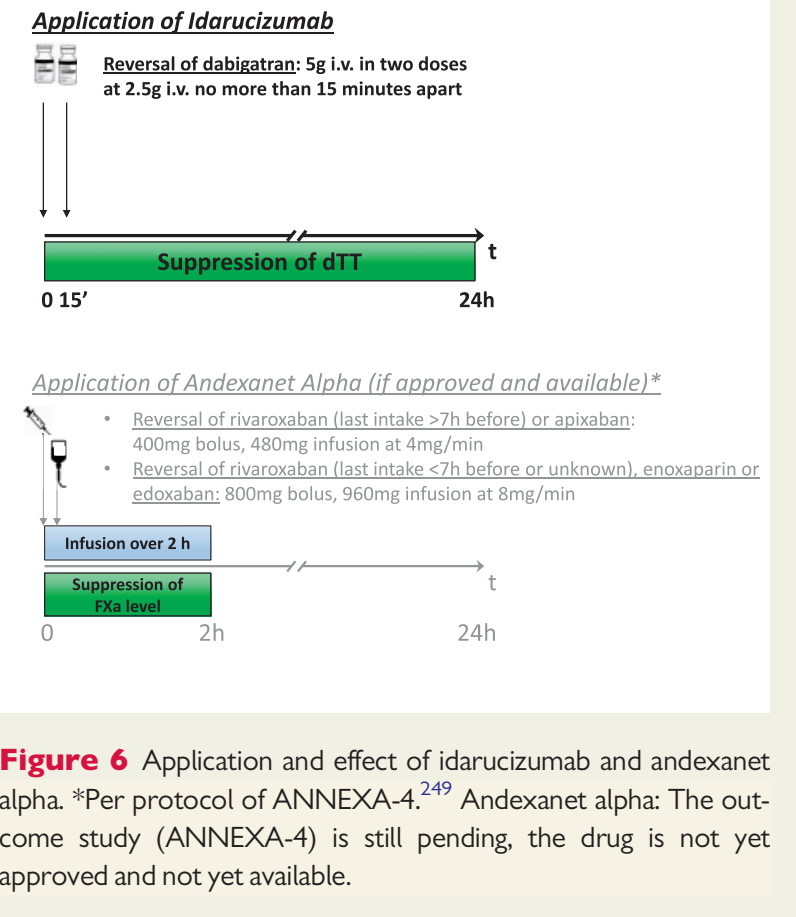

bleed due to cancer) anticoagulation can be resumed once the cause of the bleed has been eliminated. As exemplified for GI bleedings, i.e. one of the most frequently encountered bleeds, many additional factors need to be taken into consideration (Figure 7). Particularly for severe and life-threatening bleedings without a clear secondary or reversible/treatable cause the risks of re-initiating anticoagulation may outweigh the benefits. In such cases, implantation of a left atrial appendage (LAA) occluder or surgical LAA occlusion may be considered as a potential substitute for long-term anticoagulation. ${ }^{3}$ However, RCT evidence for LAA occlusion after bleeding under $\mathrm{OAC}$ is missing, which is why, ideally, treatment should occur wherever possible in the framework of a randomized trial to contribute to evidence for this difficult to treat population.

The approach post-intracerebral, intracranial, subdural, and epidural bleeding is outlined below (chapter 17).

\section{Patients undergoing a planned invasive procedure, surgery or ablation}

\section{When to stop non-vitamin $\mathrm{K}$ antagonist oral anticoagulants?}

About one quarter of anticoagulated patients require temporary cessation for a planned intervention within 2 years. ${ }^{260}$ Awaiting the results of the ongoing Perioperative Anticoagulant Use for Surgery Evaluation (PAUSE; NCT02228798) study, few prospective data on the management of NOACs are available. ${ }^{271}$ Various societies have issued separate guidelines on the timing of NOAC

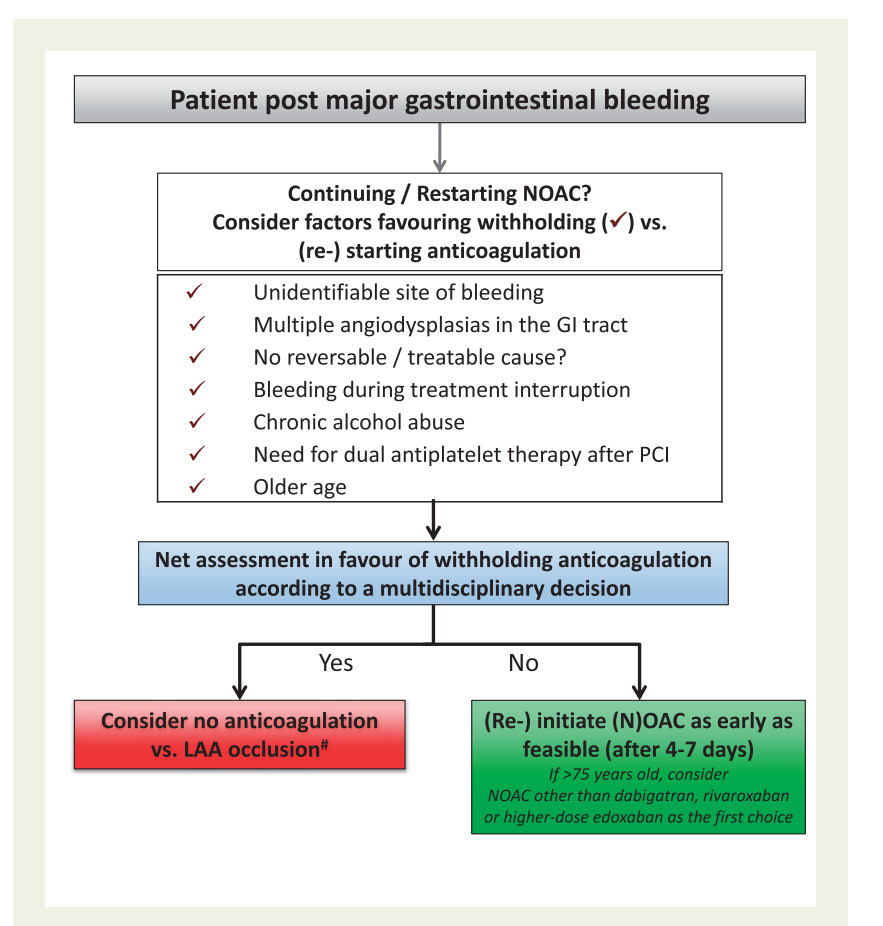

Figure 7 (Re-) initiation of anticoagulation post-gastrointestinal bleeding. "Without evidence; ideally include patient in ongoing trial.

interruption prior to surgery or interventions. It is impossible to summarize all recommendations, and healthcare providers are recommended to check this guide's recommendations against the relevant recommendations of their country/healthcare setting and professional society. The EHRA practical guide intends to provide a unified approach, which is as simplified as possible to allow its broad implementation.

Patient characteristics (including age, history of bleeding complications, concomitant medication, and kidney function) as well as surgical factors (Table 11) need to be taken into account to determine when to discontinue and restart a NOAC. While invasive surgical interventions require temporary discontinuation of a NOAC, many less invasive procedures carry a relatively low bleeding risk and do not necessarily require discontinuation (Table 12; Figure 8). All patients undergoing a planned intervention as well as caregivers (primary care physician etc.) should receive a written note indicating the anticipated date and time of their intervention as well as the date and time of the last intake of their NOAC (and any other medication).

\section{Minor bleeding risk}

It is recommended not to interrupt oral anticoagulation for most minor surgical procedures and those procedures where bleeding is easily controllable (Figure 8). In general, these procedures can be performed $12-24 \mathrm{~h}$ after the last NOAC intake. It may be practical to have the intervention scheduled $18-24 \mathrm{~h}$ after the last NOAC intake, and then restart $6 \mathrm{~h}$ later (skipping one dose of dabigatran or apixaban or no dose of edoxaban or rivaroxaban). The patient may only leave the ambulatory practice/outpatient clinic/hospital, if any periinterventional bleeding has completely stopped. Moreover, the 
Table I I Timing of last non-vitamin K antagonist oral anticoagulant intake before start of an elective intervention

\begin{tabular}{|c|c|c|c|c|}
\hline & \multicolumn{2}{|l|}{ Dabigatran } & \multicolumn{2}{|c|}{ Apixaban - Edoxaban - Rivaroxaban } \\
\hline & \multicolumn{4}{|c|}{$\begin{array}{l}\text { No important bleeding risk and/or adequate local haemostasis possible: perform at trough level } \\
\text { (i.e. } 12 \mathrm{~h} \text { or } 24 \mathrm{~h} \text { after last intake) }\end{array}$} \\
\hline & Low risk & High risk & Low risk & High risk \\
\hline $\mathrm{CrCl} \geq 80 \mathrm{~mL} / \mathrm{min}$ & $\geq 24 \mathrm{~h}$ & $\geq 48 \mathrm{~h}$ & $\geq 24 \mathrm{~h}$ & $\geq 48 \mathrm{~h}$ \\
\hline $\mathrm{CrCl} 50-79 \mathrm{~mL} / \mathrm{min}$ & $\geq 36 \mathrm{~h}$ & $\geq 72 \mathrm{~h}$ & $\geq 24 \mathrm{~h}$ & $\geq 48 \mathrm{~h}$ \\
\hline $\mathrm{CrCl} 30-49 \mathrm{~mL} / \mathrm{min}$ & $\geq 48 \mathrm{~h}$ & $\geq 96 \mathrm{~h}$ & $\geq 24 \mathrm{~h}$ & $\geq 48 \mathrm{~h}$ \\
\hline $\mathrm{CrCl} 15-29 \mathrm{~mL} / \mathrm{min}$ & Not indicated & Not indicated & $\geq 36 \mathrm{~h}$ & $\geq 48 \mathrm{~h}$ \\
\hline $\mathrm{CrCl}<15 \mathrm{~mL} / \mathrm{min}$ & \multicolumn{4}{|c|}{ No official indication for use } \\
\hline \multicolumn{5}{|c|}{ No bridging with LMWH/UFH } \\
\hline \multicolumn{5}{|c|}{ Resume full dose of NOAC $\geq 24 \mathrm{~h}$ post-low bleeding risk interventions and $48(-72) \mathrm{h}$ post-high-bleeding risk interventions (see also Figure 8 ) } \\
\hline \multicolumn{5}{|c|}{$\begin{array}{l}\text { Patients undergoing a planned intervention should receive a written note indicating the anticipated date and time of their intervention, } \\
\text { and the date and time of the last intake of their NOAC (and any other medication) }\end{array}$} \\
\hline
\end{tabular}

Low risk: with a low frequency of bleeding and/or minor impact of a bleeding; high risk: with a high frequency of bleeding and/or important clinical impact. See also Table 12. $\mathrm{CrCl}$, creatinine clearance; LMWH, low molecular weight heparin; UFH, unfractionated heparin.

patient has to be instructed about the normal post-procedural course and the measures to be taken in case of bleeding. The physician/dentist (or an informed colleague) has to be accessible in such a case.

\section{Low bleeding risk}

For invasive procedures with a low bleeding risk (i.e. low frequency of bleeding and/or minor impact of bleeding; Table 11), it is recommended to take the last dose of a NOAC $24 \mathrm{~h}$ before the elective procedure in patients with normal kidney function (Table 12, Figure 8). For patients on dabigatran and $\mathrm{CrCl}<80 \mathrm{~mL} / \mathrm{min}$ a graded interruption should be considered. For patients taking a FXa inhibitor and with a $\mathrm{CrCl}$ of $15-29 \mathrm{~mL} / \mathrm{min}$ the last NOAC should be taken $36 \mathrm{~h}$ or more before surgery (Table 12). In patients taking concomitant dronedarone, amiodarone or verapamil, it may be advisable to add an extra $24 \mathrm{~h}$ of interruption, especially if the thromboembolic risk is not very high $\left(\mathrm{CHA}_{2} \mathrm{DS}_{2}-\mathrm{VASc} \leq 3\right) .{ }^{168}$ Conversely, for some procedures (e.g. cardiac device implantations, see below) a shorter interruption may be warranted, including intake of the last dose the morning of the day before the procedure. The PAUSE trial will provide more information on the relation between last intake, preprocedural plasma level and, most importantly, clinical outcome. ${ }^{271}$

\section{High bleeding risk}

In case of invasive procedures that carry a high risk for major bleeding (i.e. with a high frequency of bleeding and/or important clinical impact), it is recommended to take the last NOAC dose $48 \mathrm{~h}$ or longer before surgery. Again, the decision to halt therapy for longer should take into account the patient's thromboembolic vs. bleeding risk as well as concomitant therapy with antiarrhythmic drugs as described above. Moreover, in patients with impaired renal function longer interruption of the NOAC intake is required, especially for dabigatran (Table 11, Figure 8). In cases with combined factors that make prediction of NOAC clearance unclear, measurement of NOAC plasma levels may be considered, and only go ahead with the planned surgical intervention when the level is considered low enough (chapter 7, Table 9). However, it needs to be clearly stated that such an approach is without evidence base, including the determination of 'safe' NOAC levels in this setting as well as waiting for levels to drop into that range whilst accepting the inherent risk of thromboembolism during that time.

\section{Bridging}

Preoperative bridging with LMWH or heparin is not recommended in NOAC-treated patients since the predictable waning of the anticoagulation effect allows properly timed short-term cessation of NOAC therapy before surgery. On the contrary, the mixing of two anticoagulants (although with similar pharmaco-dynamics and -kinetics) has been associated with an increased bleeding risk. ${ }^{272}$ As demonstrated in the BRIDGE trial for VKA, bridging with heparin/LMWH was associated with a significantly higher risk of major bleeding during cessation of oral anticoagulation but did not reduce cardiovascular events. ${ }^{273}$

\section{Dental surgery}

Dental surgery is generally considered a procedure with minor bleeding risk and with the possibility for adequate local haemostasis. Most professional statements on dental surgery advise not to suspend NOAC treatment and avoid the use of NSAIDs. ${ }^{274}$ However, recommendations are often based on a low quality of evidence and mainly rely on available pharmacological information. ${ }^{275}$ Dental extractions can generally be performed safely in an outpatient facility by applying 
Table I 2 Classification of elective surgical interventions according to bleeding risk

\begin{tabular}{|c|}
\hline Interventions with minor bleeding risk \\
\hline Dental interventions \\
\hline Extraction of $1-3$ teeth \\
\hline Paradontal surgery \\
\hline Incision of abscess \\
\hline Implant positioning \\
\hline Cataract or glaucoma intervention \\
\hline Endoscopy without biopsy or resection \\
\hline $\begin{array}{l}\text { Superficial surgery (e.g. abscess incision; small dermatologic } \\
\text { excisions; ....) }\end{array}$ \\
\hline $\begin{array}{l}\text { Interventions with low bleeding risk (i.e. infrequent or with low } \\
\text { clinical impact) }\end{array}$ \\
\hline Endoscopy with biopsy \\
\hline Prostate or bladder biopsy \\
\hline $\begin{array}{l}\text { Electrophysiological study or catheter ablation (except complex } \\
\text { procedures, see below) }\end{array}$ \\
\hline $\begin{array}{l}\text { Non-coronary angiography (for coronary angiography and ACS: } \\
\text { see Patients undergoing a planned invasive procedure, surgery } \\
\text { or ablation section) }\end{array}$ \\
\hline $\begin{array}{l}\text { Pacemaker or ICD implantation (unless complex anatomical set- } \\
\text { ting, e.g. congenital heart disease) }\end{array}$ \\
\hline $\begin{array}{l}\text { Interventions with high bleeding risk (i.e. frequent and/or with } \\
\text { high impact) }\end{array}$ \\
\hline $\begin{array}{l}\text { Complex endoscopy (e.g. polypectomy, ERCP with sphincterot- } \\
\text { omy etc.) }\end{array}$ \\
\hline Spinal or epidural anaesthesia; lumbar diagnostic puncture \\
\hline Thoracic surgery \\
\hline Abdominal surgery \\
\hline Major orthopaedic surgery \\
\hline Liver biopsy \\
\hline Transurethral prostate resection \\
\hline Kidney biopsy \\
\hline Extracorporeal shockwave lithotripsy (ESWL) \\
\hline $\begin{array}{l}\text { Interventions with high bleeding risk AND increased throm- } \\
\text { boembolic risk }\end{array}$ \\
\hline $\begin{array}{l}\text { Complex left-sided ablation (pulmonary vein isolation; some VT } \\
\text { ablations) }\end{array}$ \\
\hline
\end{tabular}

For each patient, individual factors relating to bleeding and thromboembolic risk need to be taken into account, and be discussed with the operating physician. local haemostatic measures, without interrupting anticoagulation or by just skipping the morning dose of the NOAC. ${ }^{276-279}$ Periprocedural management includes specific haemostatic techniques including the use of oxidized cellulose or absorbable gelatin sponge, sutures, tranexamic acid mouthwashes, or compressive gauze soaked in tranexamic acid.

\section{Device implantation procedures}

Device implantations are generally considered procedures with a low bleeding risk. For patients undergoing device implantation, prospective, and randomized data in VKA-treated patients have indicated lower thromboembolic and bleeding rates if the VKA is continued in an uninterrupted fashion. ${ }^{280}$ For NOAC-treated patients, the recently presented BRUISE-CONTROL 2 trial demonstrated similar bleeding and embolic rates in patients with a last intake $48 \mathrm{~h}$ before the implantation for rivaroxaban/apixaban (and based on glomerular filtration rate for dabigatran) vs. continued NOAC until the morning of the procedure (Birnie et al., presented at AHA 2017). Therefore, a standard strategy as for 'low bleeding risk' procedures with intake of the last dose in the morning of the day before the procedure can be recommended in most cases, followed by restarting one day afterwards (Table 12 and Figure 8 ). An overview of data and recommendations can be found in the recent EHRA/HRS/APHRS consensus document. $^{281}$

\section{Regional anaesthesia and pain medicine}

Invasive procedures such as spinal anaesthesia, epidural anaesthesia, and lumbar puncture require complete haemostatic function, and fall under the 'high bleeding risk' category. European as well as North American guidelines do not recommend neuraxial anaesthesia or deep blocks in the presence of uninterrupted NOAC use and recommend interruption of NOACs for up to five halflives (corresponding to an interruption of 3 days in FXa-inhibitors and 4-5 days for dabigatran). ${ }^{282,283}$ NOAC therapy can usually be resumed $24 \mathrm{~h}$ after the intervention. On the other hand, 'low risk' procedures (such as peripheral nerve blocks or peripheral joint and musculoskeletal injections) do not necessarily require NOAC interruption and if so for only a short period (e.g. two half-lives). ${ }^{284}$

\section{Lab testing before surgery or invasive procedures}

Specific coagulation measurements (see chapter 7) prior to surgery or invasive procedures provide a direct assessment of the (residual) drug concentration ${ }^{285}$ and may be useful in high-risk interventions and/or patients at risk for relevant residual drug concentrations such as older age, renal impairment, or certain concomitant medication (see chapter 5). ${ }^{168}$ However, as indicated, such an approach is without evidence base, including the determination of 'safe' NOAC levels. For the majority of patients and procedures, a 'time-based' interruption as outlined above appears safe.

\section{When to restart a non-vitamin $\mathrm{K}$ antagonist oral anticoagulant after an invasive procedure?}

After a procedure with immediate and complete haemostasis, NOACs can generally be resumed $6-8 \mathrm{~h}$ after the end of the 


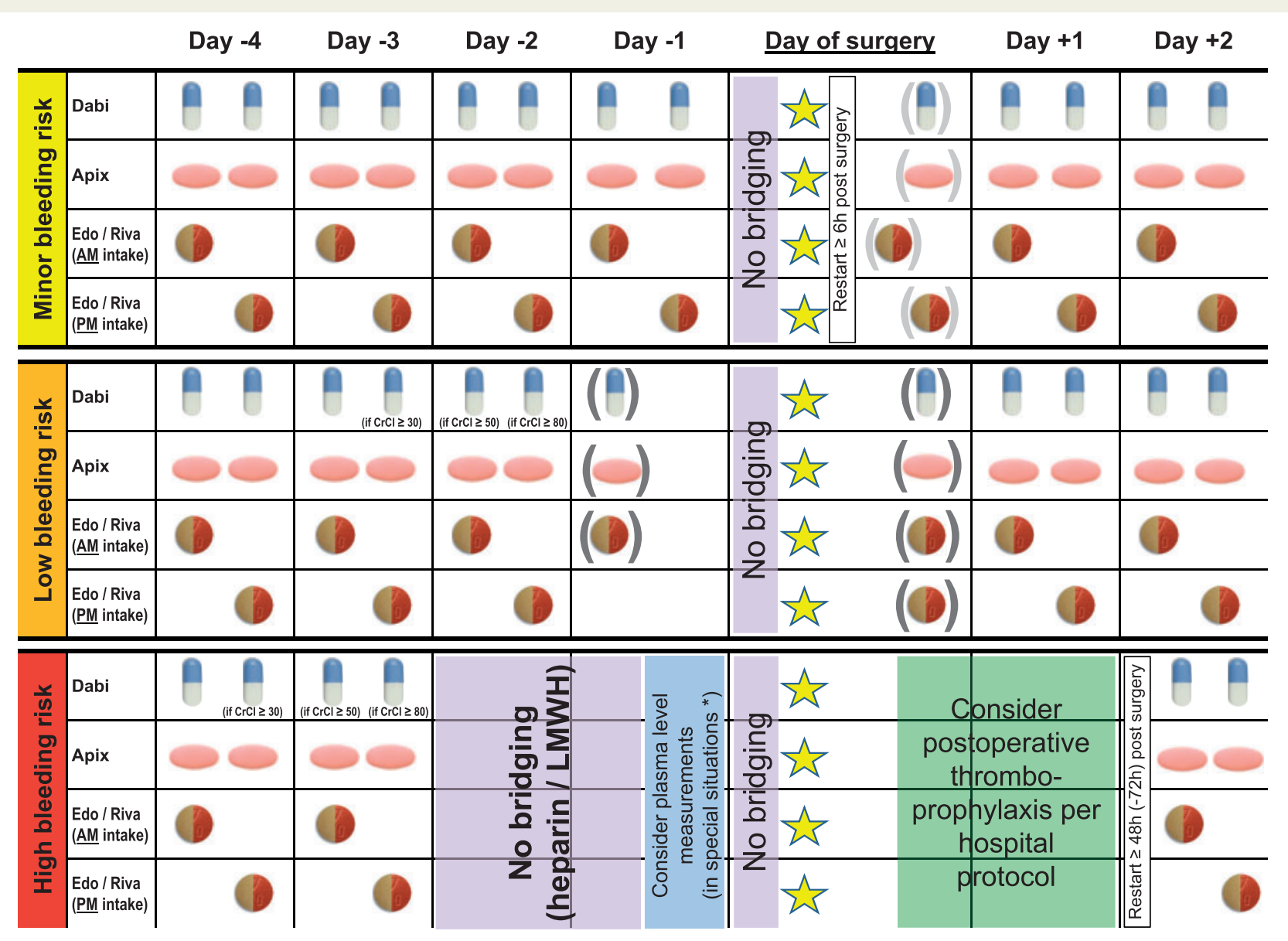

Figure 8 Stopping and re-initiation of non-vitamin K antagonist oral anticoagulant therapy in elective surgery. Yellow star, time point of the intervention/operation. Consider $+24 \mathrm{~h}$ of interruption in situations likely resulting in increased plasma levels [e.g. patients taking verapamil, body weight $<50 \mathrm{~kg}$, significant interactions (see chapter 5)]. *Consider measurement of plasma levels (see chapter $\mathbf{7}$ ) in very special situations, e.g. highest risk neurosurgery/cardiac surgery, severe renal insufficiency, and combination of factors predisposing to higher non-vitamin $\mathrm{K}$ antagonist oral anticoagulant levels (see chapter 5). Rivaroxaban needs to be taken with food for stroke prevention in atrial fibrillation, which needs to be looked after (also) in the post-operative setting. Apix, apixaban; $\mathrm{CrCl}$, creatinine clearance; Dabi, dabigatran; Edo, edoxaban; LMWH, low molecular weight heparin; Riva, rivaroxaban.

intervention. However, there are some surgical interventions in which resuming full dose anticoagulation within the first $48-72 \mathrm{~h}$ after the procedure carries a bleeding risk that may outweigh the risk of AF-related embolism. In such cases, initiation of post-operative thromboprophylaxis $6-8 \mathrm{~h}$ after surgery and restarting the NOAC 48-72 hours postoperatively (but as soon as possible) can be considered. There are, however, no data on the safety and efficacy of the post-operative use of a reduced dose of the NOACs (such as used for the prevention of venous thromboembolism (VTE) after hip/knee replacement) in patients with $\mathrm{AF}$ undergoing a surgical procedure.

It is strongly recommended to develop and implement institutional guidelines and a hospital-wide policies concerning perioperative anticoagulation management in different surgical settings, which are widely communicated and readily available.

\section{Special considerations for atrial fibrillation ablation procedures}

Left atrial catheter ablation constitutes an intervention with a risk of serious bleeding secondary to trans-septal puncture or extensive manipulation and ablation in the left atrium, although the incidence has been decreasing. ${ }^{286}$ Major bleeds in the groin are not uncommon. On the other hand, left atrial catheter ablation implies a prothrombotic setting, increasing the risk of thromboembolic complications. ${ }^{286,287}$ Recent international consensus statements and guidelines recommend performing left atrial catheter ablation under uninterrupted anticoagulant treatment (target INR 2-2.5), ${ }^{3,286}$ since such a strategy was associated with less thromboembolic events and less bleeding. ${ }^{288}$ The randomized RE-CIRCUIT (comparing dabigatran to warfarin in addition to peri-interventional heparin) ${ }^{289}$ as well as the 
VENTURE AF trial (comparing rivaroxaban to warfarin in addition to peri-interventional heparin) ${ }^{290}$ showed a similar risk of embolism in the uninterrupted NOAC vs. VKA arms, although both studies by themselves were underpowered to detect statistically significant differences in endpoints. While in VENTURE-AF, patients preferentially received their last dose rivaroxaban in the evening before the procedure, dabigatran was routinely administered even in the morning before ablation in RE-CIRCUIT. As a result, approximately $80 \%$ of patients received their last dose $<8 \mathrm{~h}$ before the procedure and $41 \%$ underwent ablation within $4 \mathrm{~h}$ of the last dabigatran dose. While a similar risk of major bleedings between rivaroxaban and warfarin was observed in VENTURE-AF, a large reduction in major bleeding was seen in RE-CIRCUIT with dabigatran compared with warfarin. Similar trials for apixaban (AXAFA-AFNET 5) ${ }^{291}$ as well as edoxaban (ELIMINATE-AF) are ongoing. Registry data as well as a subanalysis of the ENGAGE-AF trial (with varying protocols and timings of NOAC interruption) did not indicate an increased risk of stroke or bleeding for apixaban or edoxaban in the setting of AF ablation. ${ }^{292-294}$

An institutional protocol for NOAC patients undergoing AF ablation should be developed to ensure a uniform approach. Whether opting to administer the last NOAC dose shortly before the procedure (i.e. 'truly uninterrupted') or to go for a short cessation period (last NOAC dose on the day before the procedure), depends on a number of factors including renal function, $\mathrm{CHA}_{2} \mathrm{DS}_{2}$-VASc score, experience of the operator, and routine practice of heparin administration prior to (first) trans-septal puncture. ${ }^{2,281,286}$ It is reasonable to administer a last dose of NOAC $12 \mathrm{~h}$ before the start of the intervention, especially if trans-septal puncture is performed without periprocedural imaging (as is mostly the case in Europe). Especially, when adherence is uncertain over the weeks prior to the intervention, left atrial thrombus should be ruled out prior to ablation. A similar approach may be advisable if the last NOAC dose is taken $\geq 36 \mathrm{~h}$ before the intervention as the patient would be without adequate anticoagulation for a prolonged period of time as well as in patients at high risk for thromboembolism. During the ablation, intravenous heparin should be administered to achieve an ACT of 300-350 s. ${ }^{295}$ It seems reasonable to use the same target ACT levels for heparin titration in NOAC-treated patients as in patients on (uninterrupted) VKA. It has been noted that the total need for heparin and the time to target ACT was higher in some NOAC treated patients. ${ }^{290,296,297}$ This likely reflects a difference in whole blood coagulability when NOACs are stopped some time before the procedure, rather than a direct interaction between NOACs and the ACT test.

NOAC intake can be resumed $3-5 \mathrm{~h}$ after sheath removal if adequate haemostasis is established and pericardial effusion has been ruled out. ${ }^{281}$

\section{Patients requiring an urgent surgical intervention}

If an emergency intervention is required, the NOAC should be discontinued immediately. Specific management will then depend on the level or urgency (immediate, urgent, or expedite; Figure 9). ${ }^{298}$
(1) Immediate procedures (Immediate life-, limb- or organ-saving intervention, typically cardiac, vascular, and neurosurgical emergency procedures) need to be performed within minutes of the decision to operate and cannot be delayed. In these cases, reversal with idarucizumab (for dabigatran) ${ }^{248}$ should be considered, especially in moderate- to high-haemorrhagic risk procedures. ${ }^{299}$ While the REVERSE-AD trial with idarucizumab enrolled both bleeding patients as well as those requiring urgent surgery, the prospective open-label Phase III trial with andexanet alfa, a reversal agent for $\mathrm{FXa}$ inhibitors, only enrols patients experiencing an acute major bleed under therapy but not patients requiring urgent surgical interventions (Clinicaltrials.gov NCT02329327). ${ }^{249}$ After publication of the full dataset and approval of the drug (expected by the end of 2018) its usefulness in this setting needs to be re-evaluated. If specific reversal agents are not available, PCCs or aPCCs should be considered despite the lack of evidence for efficacy and safety (see also chapter 11 section). ${ }^{269,272,283}$ Especially, if no specific reversal agent is available it may be advisable to perform immediate (and urgent) procedures under general rather than spinal anaesthesia in order to reduce the risk of epidural haematoma.

(2) Urgent procedures (e.g. intervention for acute onset or clinical deterioration of potentially life-threatening conditions, conditions that may threaten the survival of limb or organ, fixation of fractures, relief of pain, or other distressing symptoms) need to be performed within hours of the decision to operate. In these situations, surgery or intervention should be deferred, if possible, until at least $12 \mathrm{~h}$ and ideally $24 \mathrm{~h}$ after the last dose. Also, coagulation test results (see below) can be awaited in this situation to gauge the necessity for reversal or application of

(a)PCCs.

(3) Expedite procedures (patients requiring early treatment where the condition is not an immediate threat to life, limb, or organ survival) should be performed within days of decision to operate. In these situations, interruption of NOACs should follow the proposed rules for elective surgery (see chapter 12).

In all such situations, particularly prior to the application of any haemostatic agents, a full panel of coagulation assays (including PT, aPTT, anti-FXa, or ITT/ECA etc.) should be obtained in order to assess the coagulation status of the patient. Even if in the emergency situation application of pro-haemostatic agents will not be postponed, results of these initial tests may have implications for further treatment during the ensuing hours. Importantly, a normal aPTT in case of dabigatran intake and a normal PT in case of rivaroxaban intake (and to a lesser extent edoxaban) may rule out high plasma levels of the respective drugs; conversely, however, normal routine coagulation tests do not exclude drug levels as expected while on therapy for all of the NOACs (see chapter 7). Specific coagulation tests (dTT or ECA for dabigatran; anti-FXa chromogenic assays for FXa inhibitors) and assessment of plasma levels may help in interpreting the current anticoagulant status as well as the waning of any anticoagulant effect, particularly in situations with potentially increased anticoagulant levels [e.g. in older age (see chapter 18.1), renal insufficiency (see chapter 6), and/or certain co-medications (see chapter 5)]. 


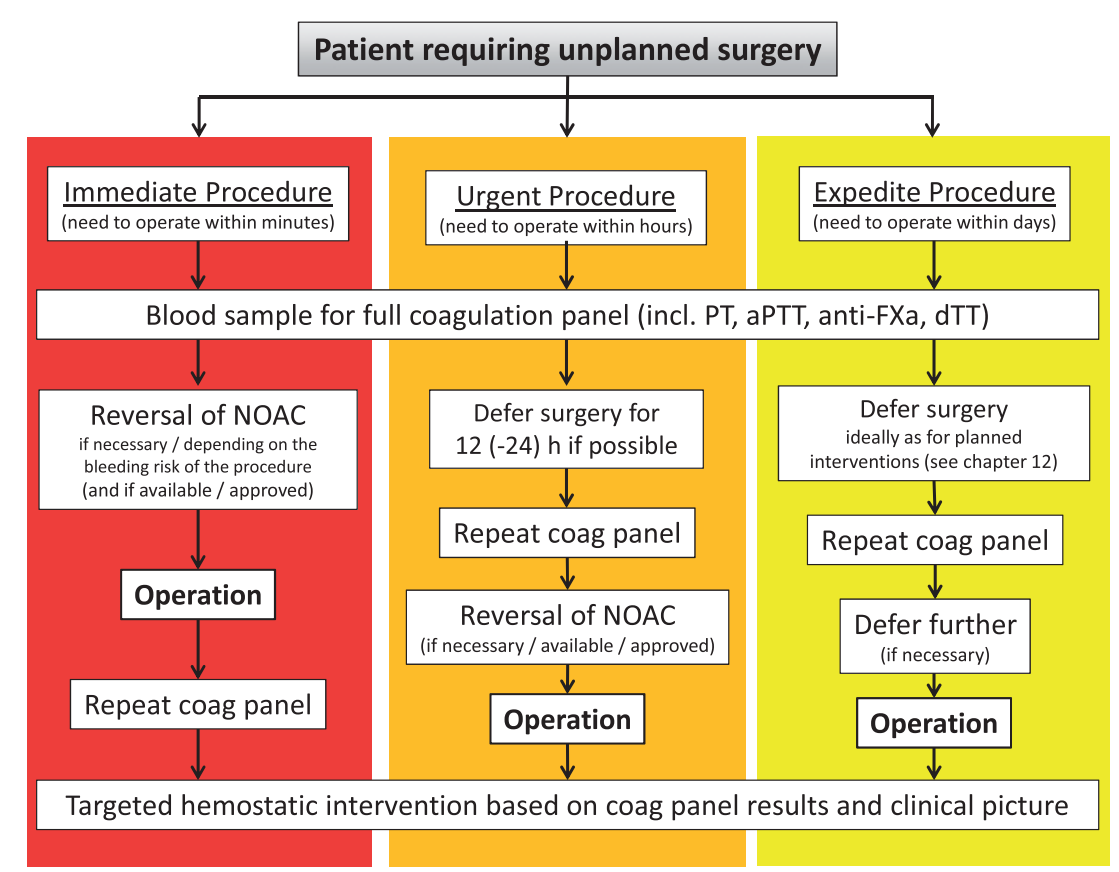

Figure 9 Non-vitamin K antagonist oral anticoagulant management in the setting of unplanned surgery.

\section{Patient with atrial fibrillation and coronary artery disease}

\section{Scope of the problem and randomized clinical trial evidence}

The combination of AF and coronary artery disease (CAD) is not only a common and complex clinical setting to deal with regarding anticoagulation and antiplatelet therapy, it is also associated with significantly higher morbidity and mortality. ${ }^{300,301}$ The practice of adding aspirin or a $\mathrm{P}_{2} \mathrm{Y}_{12}$ inhibitor to a (N)OAC is referred to as "dual therapy', while adding both aspirin and a P2Y 12 inhibitor to a (N)OAC is called 'triple therapy'. Dual antiplatelet therapy is referred to as 'DAPT'. Stacking antithrombotic agents, i.e. by adding one or two antiplatelet(s) to NOACs, inevitably increases the risk of bleeding significantly, ${ }^{170,171,302,303}$ leading to a clear need to avoid long-term triple therapy in daily clinical practice. ${ }^{304-306}$

The current understanding is that DAPT is necessary to prevent stent thrombosis but not sufficient for stroke prevention, ${ }^{307}$ and vice versa, that (N)OAC are essential for stroke prevention but on their own not suitable for preventing new coronary events, especially in the acute/subacute setting. ${ }^{3}$ A combination of at least one antiplatelet agent in addition to (N)OAC is recommended for up to 12 months after an ACS event and/or stenting procedure according to the most recent $\mathrm{ESC}$ guidelines on $\mathrm{AF}^{3}{ }^{3} \mathrm{ST}$-elevation myocardial infarction (STEMI), ${ }^{33}$ and the use of antiplatelet agents. ${ }^{32}$

To date, there are a handful of prospective trials addressing the issue of oral anticoagulation after $\mathrm{PCl}$, including two RCTs comparing NOACs to VKA, in a variety of combinations with antiplatelet agents. ${ }^{141,308}$ In essence, these trials focus on bleeding as the primary endpoint, and are underpowered to address relatively rare ischaemic/thromboembolic events including stroke, re-infarction and stent thrombosis. A meta-analysis combining WOEST, PIONEER AF-PCI, and RE-DUAL PCl suggests that the likelihood of an excess of thromboembolic events during dual therapy vs. triple therapy is low. ${ }^{309}$ The two ongoing NOAC in AF trials, AUGUSTUS (NCT02415400) and ENTRUST-AF PCI (NCT02866175) ${ }^{310}$ will add further information on how and how long (if at all) triple anticoagulation should be administered.

\section{Randomized clinical trial evidence for non-vitamin K antagonist oral anticoagulants post-percutaneous coronary intervention}

In PIONEER AF-PCl, two different rivaroxaban regimens were compared with 'standard' triple therapy with VKA and DAPT in 2124 AF patients undergoing PCl: a low-dose of rivaroxaban $15 \mathrm{mg}(10 \mathrm{mg}$ in patients with $\mathrm{CrCl} 30-50 \mathrm{~mL} / \mathrm{min}$ ) with a $\mathrm{P}_{2} \mathrm{Y}_{12}$ inhibitor and a very low dose of rivaroxaban $2.5 \mathrm{mg}$ twice daily combined with aspirin and a $\mathrm{P}_{2} \mathrm{Y}_{12}$ inhibitor. ${ }^{311}$ The trial design was complex: One year fixed treatment of $15 \mathrm{mg}$ rivaroxaban plus $\mathrm{P}_{2} \mathrm{Y}_{12}$ inhibitor was compared to triple anticoagulation with very-low dose rivaroxaban $(2 \times 2.5 \mathrm{mg})$ or VKA. The P2Y 12 inhibitor was clopidogrel in the vast majority of patients, and DAPT durations of 1,6 , and 12 months were pre-specified for the latter two arms. PIONEER AF-PCI showed that both rivaroxaban arms reduced the risk of clinically significant bleeding complications at 1 year when compared with standard triple therapy with a VKA targeted to an INR between 2 and 3 and with 
varying DAPT durations. ${ }^{308}$ While there were numerically similar rates of cardiovascular death, myocardial infarction, or stroke in all three arms, the trial was underpowered for efficacy. However, neither of the rivaroxaban doses in PIONEER AF-PCl $(15 \mathrm{mg} / 10 \mathrm{mg}$ OD or $2.5 \mathrm{mg} \mathrm{BID}$ ) have been investigated for stroke prevention in $\mathrm{AF}$ [with the exception of the $15 \mathrm{mg}$ dose in a relatively underpowered trial conducted in an exclusively Japanese population with normal renal function (J-ROCKET)]. ${ }^{156}$

In RE-DUAL PCl, the safety of two doses of dabigatran (110 or $150 \mathrm{mg}$ BID) in combination with clopidogrel or ticagrelor (i.e. dual therapy, without aspirin) were compared with standard triple therapy (for 1 or 3 months depending on the type of stent) with VKA, aspirin, and either clopidogrel or ticagrelor in 2725 patients with $\mathrm{AF}$ undergoing PCl. ${ }^{141}$ The composite of major or clinical relevant nonmajor bleeding events and major bleeding events alone were significantly reduced in the 110- and 150-mg dabigatran dual therapy arms compared to the standard VKA triple therapy arm. This trial was also underpowered for individual efficacy endpoints; however, it was powered to show non-inferiority of the combined dual-therapy arms vs. the triple therapy in a composite efficacy endpoint of death, thromboembolic events and unplanned revascularization. Stent thrombosis was observed in $15(1.5 \%)$ patients in the $110-\mathrm{mg}$ dual therapy group vs. $8(0.8 \%)$ patients in the triple-therapy group $(P=0.15)$ and in $7(0.9 \%)$ patients in the 150-mg dual-therapy group. ${ }^{141}$ Both dabigatran doses in RE-DUAL PCl have been shown non-inferior $(110 \mathrm{mg})$ or superior $(150 \mathrm{mg})$ to VKA for stroke prevention in $\mathrm{AF}^{28}$

\section{Key 'scientific' data on the use of non- vitamin $\mathrm{K}$ antagonist oral anticoagulants in patients with atrial fibrillation and acute coronary syndrome, percutaneous coronary intervention, or stable coronary artery disease}

\section{What is known:}

(1) Adding aspirin and/or a $\mathrm{P}_{2} \mathrm{Y}_{12}$ inhibitor to oral anticoagulants substantially increases bleeding risk across different clinical scenarios and should thus be avoided in AF patients without clear indication for antiplatelet therapy, including CAD patients beyond 12 months after an ACS. ${ }^{170,300,312}$ However, in general the bleeding risk seems to be lower with a NOAC plus antiplatelet combination than with a VKA plus antiplatelet combination. ${ }^{170,302,313}$

(2) ESC guidelines clearly state that the length of DAPT does not depend (anymore) on the type of stent (i.e. DES or BMS) but on the clinical presentation of the patient. ${ }^{3,32}$ As contemporary DES are more efficient and as safe (or safer) as BMS regarding the risk for stent thrombosis, it does not make sense to opt for a BMS as a strategy to reduce the duration of $\mathrm{P}_{2} \mathrm{Y}_{12}$ inhibitor therapy in patients on a NOAC. The use of a contemporary DES will also minimize the risk of avoidable repeat interventions due to restenosis thereby reducing the need for additional periods of dual or triple therapy.

(3) Clinical trials with contemporary DES suggest that (very) short dual antiplatelet regimens (i.e. 1 month after elective stenting or 6 months in case of ACS) are safe and efficacious in patients perceived to have a high bleeding risk and/or the elderly. ${ }^{314,315}$ Patients receiving (N)OAC in combination with dual antiplatelet agents are considered to be at high bleeding risk.

(4) Rivaroxaban $15 \mathrm{mg}$ or dabigatran $110 / 150 \mathrm{mg}$ BID in dual therapy with $\mathrm{P}_{2} \mathrm{Y}_{12}$ inhibitor, mainly clopidogrel (but without aspirin) is safer in terms of bleeding risk than triple therapy with VKA, clopidogrel, and low-dose aspirin (PIONEER AF-PCI / RE-DUAL PCI). ${ }^{141,308}$

(5) Rivaroxaban $2.5 \mathrm{mg}$ BID in triple therapy with aspirin and clopidogrel is safer in terms of bleeding risk than triple therapy with doseadjusted VKA, clopidogrel, and low-dose aspirin.

(6) Measures to reduce the bleeding risk in patients with ACS should be retained: low doses of aspirin (75-100 mg), especially when combined with a P2Y 12 inhibitor; new-generation drug-eluting stents (DES) to minimize the duration of dual/triple therapy; and a radial approach for interventional procedures (reducing at least the risk of access site bleeding). ${ }^{33,316}$

(7) Prolonged antiplatelet therapy beyond 1 year after ACS or DES implantation has been suggested in non-(N)OAC treated patients based on large-scale RCTs. ${ }^{317-319}$ In the DAPT trial, patients were randomized 12 months after a $\mathrm{PCl}$ with DES to aspirin plus clopidogrel or aspirin alone, up to 30 months after the PCl. In the PEGASUS TIMI 54 trial, patients were randomized 1-3 years after an myocardial infarction to aspirin plus ticagrelor or aspirin alone, and followed for a median of 33 months. Since patients in need of long-term OAC therapy were excluded from these studies, the results are of less relevance for treatment of AF patients.

\section{What is unknown}

(1) It is unknown whether the doses of rivaroxaban used in PIONEER AF-PCl (i.e. $2.5 \mathrm{mg}$ BID or $15 \mathrm{mg} O D$ ) are sufficient for stroke prevention, at least compared with standard dose-adjusted VKA or compared with the $20 \mathrm{mg}$ OD rivaroxaban dose in patients with a normal renal clearance. ${ }^{29}$

(2) It remains unknown whether dual therapy strategies combining a NOAC with clopidogrel are safer in terms of bleeding risk than a dual therapy with a VKA and clopidogrel. This is currently being addressed in the AUGUSTUS study with apixaban.

(3) It remains unknown whether dual therapy (i.e. rivaroxaban $15 \mathrm{mg}$ OD or dabigatran $110 / 150 \mathrm{mg}$ BID in combination with a P2Y 12 inhibitor) sufficiently protects against stent thrombosis or myocardial infarction, due to underpowered clinical trials. ${ }^{141,308}$

(4) It remains unknown whether dual therapy with $N O A C$ and aspirin could be an alternative to NOAC and a P2Y 12 inhibitor, as there is no randomized study evaluating aspirin vs. a $\mathrm{P}_{2} \mathrm{Y}_{12}$ inhibitor as part of dual therapy with NOAC or VKA.

(5) There were insufficient numbers of patients on ticagrelor or prasugrel in both PIONEER AF-PCl and RE-DUAL PCI to conclusively assess the safety of combining these more powerful $\mathrm{P}_{2} \mathrm{Y}_{12}$ inhibitors in dual or triple therapy regimens.

(6) In VKA-treated patients, a $\mathrm{PCl}$ seems safe without bridging and without additional periprocedural heparin. ${ }^{320}$ It is unknown if this applies also to NOACs, since most clinical studies have suggested interruption of NOAC therapy at $\mathrm{PCl}$. A small pilot study in 50 stable patients undergoing planned $\mathrm{PCl}$ and on DAPT suggests that pre-procedural dabigatran provides insufficient anticoagulation during $\mathrm{PCl}^{321}$ 
A similar study with rivaroxaban, however, showed suppressed coagulation activation after elective $\mathrm{PCl}$, without increased bleeding. ${ }^{322}$ The safety of performing a $\mathrm{PCl}$ in patients on a NOAC, with or without additional periprocedural intravenous anticoagulation still needs to be prospectively studied in larger clinical trials.

\section{Scenario 1: coronary interventions in patients with known atrial fibrillation already on non-vitamin $\mathbf{K}$ antagonist oral anticoagulant}

Whereas guidelines recommend maintaining VKA patients uninterrupted on their treatment, both during elective or urgent $\mathrm{PCl}$, NOACs should preferably be temporarily discontinued for elective interventions and upon presentation with non-ST-elevation ACS where early coronary angiogram is anticipated, as has been done during the pivotal NOAC vs. VKA AF trials. NOACs should be continued in non-invasively-managed ACS patients. Performing a $\mathrm{PCl}$ (scheduled or not) under NOAC is different than under VKA for many reasons: last dose and adherence needs to be carefully scrutinized; uncertainty about the extent of anticoagulation in the absence of mainstream/point of care tests, and hence uncertainty about stacking of additional periprocedural anticoagulants; variability in renal function (especially when unknown in an acute setting); singular antifactor II or Xa blockade vs. multifactor antagonism with VKA, etc. Temporary discontinuation of the short-acting NOACs allows safe initiation of antiplatelet therapy and standard local anticoagulation practices periprocedurally.

In the 2016 ESC AF guideline and 2017 DAPT focused update, the use of ticagrelor or prasugrel as part of a triple therapy regimen is discouraged (Class III, level of evidence C), but no comments are made on dual therapy with combination of ticagrelor or prasugrel and a NOAC as possible alternative for triple therapy with aspirin, clopidogrel and a NOAC.,32 It leaves the opportunity to use one of these newer $\mathrm{P}_{2} \mathrm{Y}_{12}$ inhibitors with a (N)OAC under certain circumstances such as perceived high thrombotic risk, ACS, or prior stent thrombosis. In a subset of the RE-DUAL PCl study the use of ticagrelor appeared safe and effective in the setting of dual therapy (Oldgren et al., presented at AHA 2017). Triple anticoagulation with any of the new $\mathrm{P}_{2} \mathrm{Y}_{12}$ inhibitors, on the other hand, is clearly discouraged beyond the first day(s) post-PCl. A signal for a relevant role of 'clopidogrel resistance' has so far not surfaced clinically in the large outcome trials but experience in earlier DAPT studies may provide a rationale for further studies on the use of newer $\mathrm{P}_{2} \mathrm{Y}_{12}$ inhibitors in the setting of dual anticoagulation.

\section{In-hospital management}

A general flow diagram indicating possible scenarios is provided in Figure 10.

\section{Elective coronary intervention (stable coronary artery disease)}

Contemporary DES are preferred to shorten exposure to dual or triple therapy after the procedure (see below) but also to avoid the need for repeat interventions. There is no reason anymore to opt for a BMS as a strategy to reduce DAPT duration. ${ }^{32,314}$ Sole balloon angioplasty or bypass surgery should be considered as an alternative in patients in need for chronic anticoagulation due to the reduced need for long-term dual or triple therapy.

There is no rationale for switching a NOAC to VKA after (or just prior) to elective $\mathrm{PCl}$, since this may be associated with an increased bleeding and thromboembolic risk compared with restarting the NOAC.

NOAC therapy should be discontinued before patients are taken to the cath lab and the procedure be performed at least (12-)24 h after last intake (see chapter 12). Periprocedural anticoagulation should be used per local practice. Unfractionated heparin $(70 \mathrm{IU} / \mathrm{kg})$ or bivalirudin rather than enoxaparin is preferred. ${ }^{323}$ Unfractionated heparin should be administered to target ACT or aPTT levels per standard clinical practice. Bivalirudin may be an alternative because of its very short therapeutic half-life.

\section{Acute coronary syndrome}

In the absence of contraindications, all NOAC patients developing an ACS should receive low-dose aspirin immediately at admission (150 $300 \mathrm{mg}$ loading dose) as well as a P2Y 12 inhibitor. Since clopidogrel as well as the newer P2Y 12 inhibitors take considerable time to achieve their maximal antiplatelet effect in unstable patients, P2Y $_{12}$ inhibition without aspirin cannot be recommended in the acute setting. In frail patients at high bleeding risk, aspirin only might be a safer initial therapy awaiting invasive management, when indicated.

ST-elevation myocardial infarction. In case of a STEMI, primary PCI via a radial approach is strongly recommended over fibrinolysis. ${ }^{324}$ It is recommended to use additional parenteral anticoagulation (i.e. UFH, enoxaparin, or bivalirudin, but not fondaparinux), regardless of the timing of the last dose of NOAC. Unless used for bail-out situations, routine glycoprotein Ilb/llla inhibitors should be avoided.

If fibrinolysis is the only available reperfusion therapy, it may be considered if the NOAC-treated patient presents with normal $\mathrm{dTT}$, ECT, aPTT (for dabigatran), PT (for FXa inhibitors), and importantly, plasma levels below the reference range (Table 9). Also, additional UFH or enoxaparin in addition to fibrinolysis should be avoided until the NOAC effect has decreased ( $12 \mathrm{~h}$ or longer after last intake).

Non-ST-elevation myocardial infarction. After discontinuing the NOAC and awaiting the waning of its effect ( $12 \mathrm{~h}$ or longer after last intake; chapter 12), fondaparinux or enoxaparin can be initiated. The use of upstream glycoprotein Ilb/llla inhibitors should be avoided in this setting. Unfractionated heparin or bivalirudin is only recommended in bail-out situations, awaiting an intervention (Class Illb C). ${ }^{325}$ To reduce the risk of access site bleeding, a radial approach is preferred. $^{324}$

In more urgent situations, the same approach as in primary $\mathrm{PCl}$ STEMI patients should be followed, as described above.

\section{Post-procedural resumption of anticoagulation}

In stabilized patients (i.e. no recurrent ischaemia or need for other invasive treatments), anticoagulation can be restarted as soon as parenteral anticoagulation has been stopped. There are no data to recommend switching to VKA (which may even be associated with higher bleeding and thromboembolic risks, especially in VKA-naive patients in whom the correct VKA dose is unknown). The same applies for AF patients after coronary bypass grafting. 


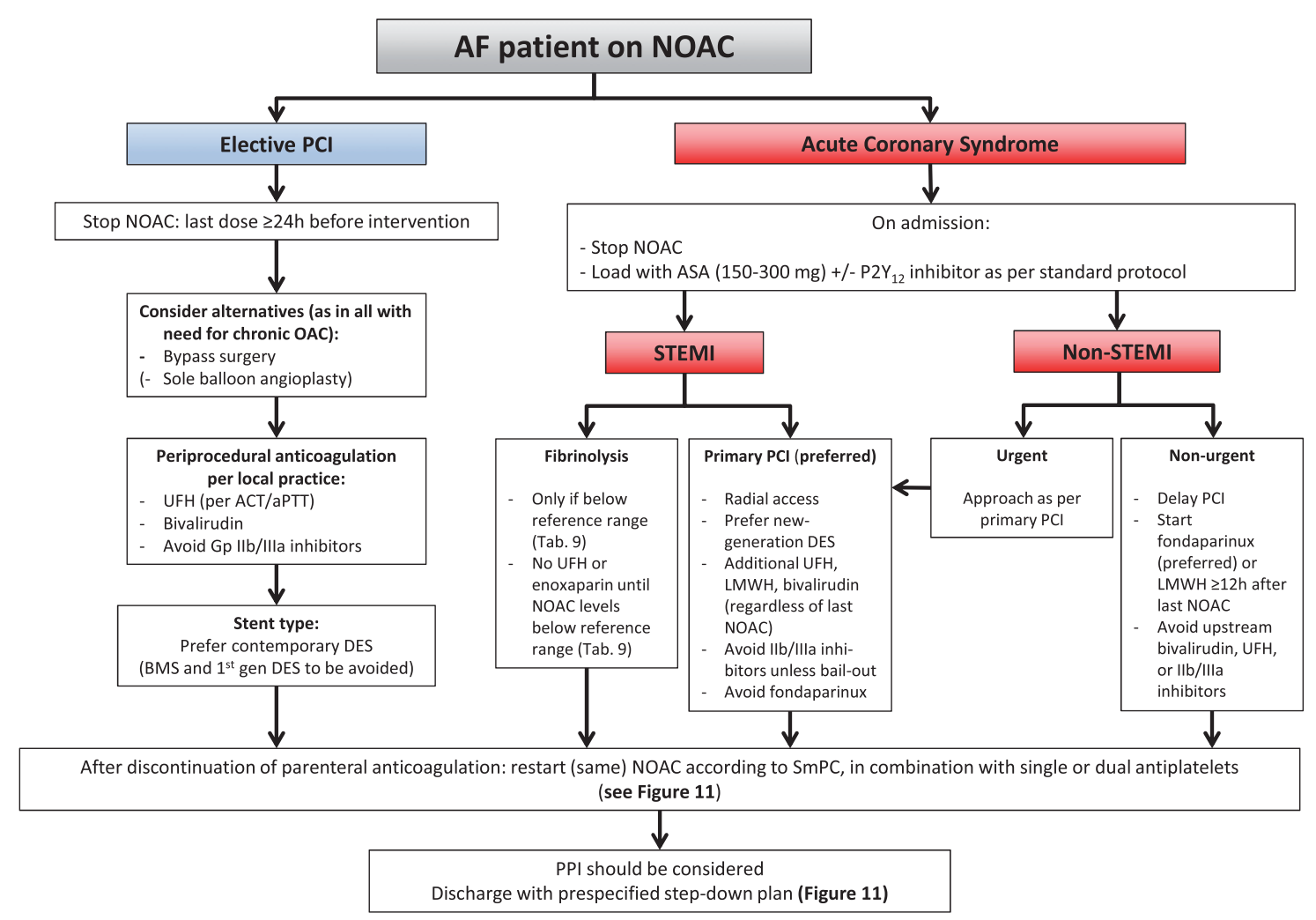

Figure I 0 Acute management of elective percutaneous coronary intervention or acute coronary syndrome in atrial fibrillation patients treated with non-vitamin $\mathrm{K}$ antagonist oral anticoagulant.

The initial combination of antiplatelet agent(s) and NOAC as well as the subsequent duration of aspirin or $\mathrm{P}_{2} \mathrm{Y}_{12}$ inhibitor treatment need to be individualized, based on a careful assessment of ischaemic vs. bleeding risk (Figure 11). Based on PIONEER AF-PCI and REDUAL PCl, triple treatment should be kept as short as possible (see chronic phase below). An alternative is to opt for dual therapy with only a NOAC and a $\mathrm{P}_{2} \mathrm{Y}_{12}$ inhibitor within 1-7 days after the acute phase.

While awaiting the results of trials with apixaban and edoxaban the $150 \mathrm{mg}$ dabigatran dual therapy appears to be the preferred choice over triple therapy for the majority of patients based on both the results from RE-LY ${ }^{28}$ and RE-DUAL $P \mathrm{Pl}^{141}$; dual therapy using $110 \mathrm{mg}$ dabigatran or rivaroxaban $15 \mathrm{mg}$ ( $10 \mathrm{mg}$ in renal insufficiency) appears as a viable alternative for patients with estimated high bleeding risk - provided that dabigatran or rivaroxaban per se appear as a good choice for this individual patient based on age (see chapter 18.1), comorbidities (e.g. renal insufficiency; see chapter 6), interactions (see chapter $\mathbf{5}$ ), and others.

\section{Management from discharge to 1 year post-acute} coronary syndrome/percutaneous coronary intervention Combining one or two antiplatelet agents with chronic anticoagulation (NOAC or VKA) significantly increases bleeding risk, regardless of the large varieties of possible combinations. ${ }^{141,170,300,302,308,326}$ Despite two recent studies on dual or triple therapy with NOAC (and two more underway), there is no one combination fitting every patient. The type and level of anticoagulation as well as one or two antiplatelet agents and its duration need to be highly individualized, based on atherothrombotic risk, cardioembolic risk, and bleeding risk. ${ }^{3,32,33}$ It is highly recommended to formally assess stroke and ischaemic event risk using validated tools such as the $\mathrm{CHA}_{2} \mathrm{DS}_{2}$-VASc and GRACE scores. ${ }^{32}$ Estimating the bleeding risk should lead to efforts to correct or reduce reversible bleeding risk factors. ${ }^{3}$ Reducing the time exposed to triple or even dual therapy needs to drive the physician's choice between the myriad of possible combinations for long-term therapy. Proton pump inhibitors should be encouraged in all patients with a combination of antiplatelets and anticoagulants, particularly in the setting of triple anticoagulation.

In patients at high ischaemic risk (e.g. after an ACS), a default time of triple therapy of 1 month up to 6 months is proposed, thereafter stepping down to dual therapy (with NOAC and either aspirin or clopidogrel) until 1 year. ${ }^{32}$ Triple therapy beyond 6 months after $\mathrm{PCl}$ is not recommended, and (much) shorter regimens will likely suffice for most patients. Factors that weigh in to shorten triple therapy with earlier switch to dual therapy are an estimated low atherothrombotic 


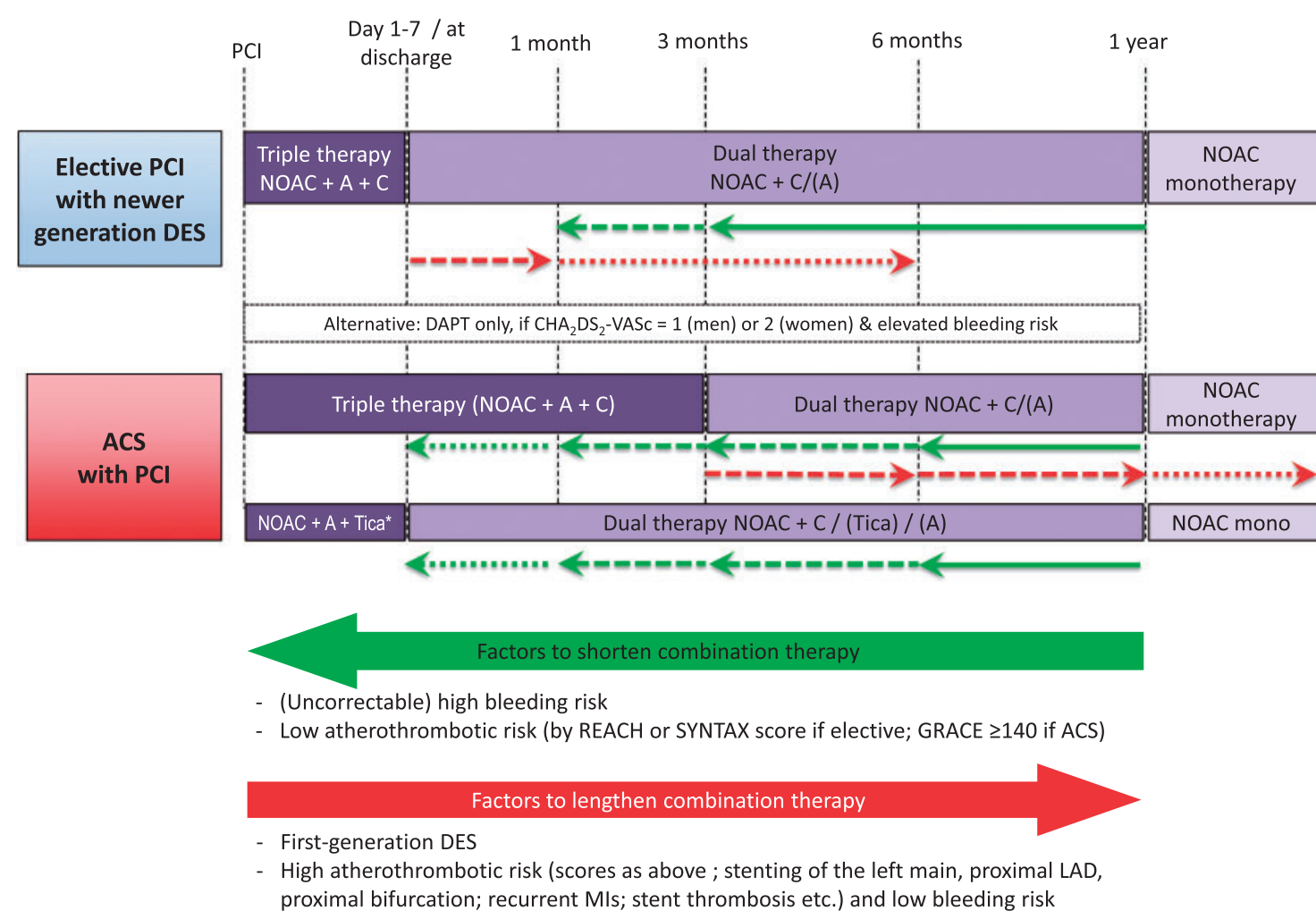

Figure I I Long-term treatment of patients on non-vitamin K antagonist oral anticoagulant therapy after elective percutaneous coronary intervention or acute coronary syndrome. There are innumerable possible variations on this global theme, as discussed in the text. Patient characteristics and institutional practices should be taken into account to individualize the approach to each and every single patient. This figure wants to create a "backbone' as guidance for such tailored approaches. A: aspirin 75-100 mg OD; C: clopidogrel $75 \mathrm{mg}$ OD; Tica: Ticagrelor $90 \mathrm{mg}$ BID. *If triple therapy needs to be continued after discharge clopidogrel is preferred over ticagrelor (due to lack of data).

risk or a high (uncorrectable) bleeding risk. Conversely, procedural and/or anatomical factors may drive longer triple therapy regimens. Beyond those patients at very high ischaemic risk, early dual therapy may well become the default strategy for most patients based on PIONEER AF-PCI and RE-DUAL PCI (while awaiting results from AUGUSTUS and ENTRUST-AF PCI). ${ }^{32,310}$

In a small subset of patients with a low stroke risk $\left(\mathrm{CHA}_{2} \mathrm{DS}_{2^{-}}\right.$ VASc of 0-1 in males or 1-2 in females, i.e. only ACS) and elevated bleeding risk, one could opt to treat with DAPT only, without anticoagulants, from the onset. ${ }^{307}$

\section{Chronic coronary artery disease setting ( $\geq 1$ year post- acute coronary syndrome/percutaneous coronary intervention)}

The 2017 ESC DAPT and 2016 AF guidelines recommend discontinuing any antiplatelet agent at 12 months after a PCl or ACS episode (see following paragraphs) and to only consider keeping one antiplatelet plus a (N)OAC beyond 12 months in patients at very high risk of coronary events. ${ }^{3,32}$ Switching to NOAC monotherapy at an earlier stage (e.g. at 6 months) could represent an alternative for patients at low ischaemic- and high bleeding risk after a $\mathrm{PCl}$ for stable angina.
Independent of the chosen anticoagulation regimen and timing, the patient needs to be discharged with a pre-specified planned downgrade schedule of antithrombotic/antiplatelet agents to reduce the longer-term risk of bleeding while protecting against coronary events. Such a schedule should be prominently delineated in the discharge letter, and reviewed at every following patient visit.

\section{Scenario 2: management of the patient with a recent acute coronary syndrome ( $<1$ year) who develops new-onset atrial fibrillation}

Current ACS guidelines recommend DAPT for up to 1 year after the acute event in patients without indication for OAC, while high-risk patients might require an even longer DAPT duration. ${ }^{318,319}$ They do, however, also allow for shorter DAPT durations (3-6 months) in high bleeding risk ACS patients. ${ }^{32,33,327}$ If AF develops during the first year after an ACS and there is an indication for thromboembolic prevention with anticoagulation, (N)OAC should be started and the need for continuing DAPT carefully weighed against the increased bleeding risk. Following a scheme as outlined above (Management from discharge to 1 year post-ACS/PCI) appears reasonable in this setting. 


\section{Scenario 3: a stable coronary artery disease patient (acute coronary syndrome $\geq 1$ year ago) develops atrial fibrillation}

Stable CAD patients developing AF should receive anticoagulation, depending on their $\mathrm{CHA}_{2} \mathrm{DS}_{2}$-VASc score. Based on studies showing that VKAs alone are superior to aspirin post-ACS, and VKAs plus aspirin may not be more protective but associated with excess bleeding, anticoagulation only without additional antiplatelet agents is considered sufficient for most AF patients with stable CAD. ${ }^{32,316,328}$

In the four Phase III NOAC AF trials, about one third of the patients had CAD and $15-20 \%$ of patients had a prior $\mathrm{MI}^{28-31}$ No interaction in terms of efficacy or safety was observed between patients with or without a prior Ml, although it is unclear in how many patients antiplatelet therapy was maintained and for how long. It is likely that the advantages of NOACs (in monotherapy) over VKAs are preserved in CAD patients with AF. Also for dabigatran, the net clinical benefit was maintained and total myocardial ischaemic events were not increased, which was further supported by the very large registry follow-up in 134000 older patients treated with dabigatran or VKA, which did not reveal any increased risk for MI. ${ }^{79,329}$ Since direct comparative data are lacking, there is no strong argument for choosing one NOAC over another in this setting based purely on the existence of stable coronary artery disease.

\section{Avoiding confusion with non-vitamin $\mathrm{K}$ antagonist oral anticoagulant dosing across indications}

In order to replicate the positive findings of the RCTs, using the correct dosing is critical, especially since all NOACs are also studied in other indications. With four NOACs available in different dosages for different indications and with different dose reduction criteria, identification of the correct dose has become more complicated and is one of the key challenges in the daily use and individualization of treatment.

Table 13 gives an overview of the currently available NOACs and their doses in the different populations and indications, including the relevant dose reduction criteria for each NOAC and indication.

\section{Cardioversion in a non-vitamin $K$ antagonist anticoagulant- treated patient}

Based on current ESC guidelines, ${ }^{3}$ in patients with $\mathrm{AF}$ of $\geq 48 \mathrm{~h}$ (or unknown) duration undergoing electrical or pharmacological cardioversion, effective oral anticoagulation needs to be established for at least 3 weeks prior to cardioversion or transesophageal echocardiography (TOE) performed to rule out left atrial thrombi. After cardioversion, continuous oral anticoagulation is mandatory for at least another 4 weeks, irrespective of $\mathrm{CHA}_{2} \mathrm{DS}_{2}$-VASc score. ${ }^{3,348}$ Different scenarios have to be distinguished: electrical cardioversion in a patient who is on chronic treatment with a NOAC and now requires cardioversion for a new bout of $\mathrm{AF}$, and cardioversion in a patient newly diagnosed with $\mathrm{AF}$ and naïve to anticoagulation (Figure 12).

\section{Cardioverting an atrial fibrillation patient treated for $\geq \mathbf{3}$ weeks with non- vitamin $\mathrm{K}$ antagonist oral anticoagulant}

Analyses from RE-LY (dabigatran), ROCKET-AF (rivaroxaban), and ARISTOTLE (apixaban) suggest that electrical cardioversion in patients treated with NOACs has a similar (and very low) thromboembolic risk as under warfarin. ${ }^{28-30}$ Later prospective trials with rivaroxaban (X-VeRT), ${ }^{349}$ edoxaban (ENSURE-AF), ${ }^{350}$ and apixaban (EMANATE, Ezekowitz et al., presented at ESC 2017) have confirmed the low peri-cardioversion stroke risk in patients treated with a NOAC for $\geq 3$ weeks compared with warfarin. These trials did not include sufficient patient numbers to demonstrate statistically sound non-inferiority. In congregate, however, these data indicated that a cardioversion without TOE seems reasonably safe under regular and continued NOAC intake, provided that adequate anticoagulation has been installed for 3 weeks before cardioversion. ${ }^{3}$ As there is no coagulation assay available for any NOAC that provides information on effective anticoagulation over the past 3 weeks, the patient needs to be inquired about adherence over the last weeks and his/her answer documented in their file. If in doubt about adherence, a TOE should be performed prior to cardioversion under a NOAC. Importantly, it has to be kept in mind that left atrial thrombi can also form in spite of adequate long lasting oral anticoagulation with a VKA or NOAC. Therefore, it remains an individual decision whether to perform a cardioversion with or without prior TOE. For this decision, the individual thromboembolic risk of a patient according to the $\mathrm{CHADS}_{2}$ or $\mathrm{CHA}_{2} \mathrm{DS}_{2}$-VASc score can be considered: in 1.6-2.1\% of therapeutically anticoagulated patients a TOE prior to AF ablation revealed thrombi or sludge in the left atrium, with the risk of thrombus correlating with the $\mathrm{CHADS}_{2}$ score (thrombus incidence $\leq 0.3 \%$ in $\mathrm{CHADS}_{2}$ 0-1 patients, thrombus incidence $0.5 \%$ in $\mathrm{CHADS}_{2} \geq 2$ patients). ${ }^{351-353}$

\section{Cardioverting atrial fibrillation of $>\mathbf{4 8} \mathrm{h}$ in a patient not on non-vitamin $K$ antagonist oral anticoagulant}

For the scenario of cardioversion in an AF patient who is not on NOAC, the X-VeRT, ${ }^{349}$ ENSURE-AF, ${ }^{350}$ and EMANATE (presented at ESC 2017) ${ }^{354}$ studies with rivaroxaban, edoxaban, and apixaban, respectively, offered important data since they included 57\%, 27\%, and $100 \%$ of OAC-naive patients, respectively. The cardioversion strategy was either early (with TOE) or without TOE (delayed strategy, i.e. with 3-8 weeks anticoagulation before cardioversion). OACnaive patients tended to have slightly higher thromboembolic event rates (which was not statistically significant). Overall, there was no difference in ischaemic or bleeding events between NOAC and VKA groups (except for lower ischaemic events with apixaban in the EMANATE trial), nor between early and delayed groups, although neither of the trials were powered for non-inferiority. In EMANATE, about half of the patients received an initial loading dose of $10 \mathrm{mg}$ (followed by $5 \mathrm{mg} \mathrm{BID);} \mathrm{also} \mathrm{these} \mathrm{patients} \mathrm{did} \mathrm{not} \mathrm{show} \mathrm{a} \mathrm{higher}$ 
Table I 3 NOACs and approved/studied doses across indications

\begin{tabular}{|c|c|c|}
\hline \multicolumn{3}{|c|}{ Stroke prevention in atrial fibrillation (SPAF) } \\
\hline & Standard dose & Comments/dose reduction \\
\hline Apixaban ${ }^{30}$ & $2 \times 5 \mathrm{mg}$ & $\begin{array}{l}2 \times 2.5 \mathrm{mg} \text { if two out of three: weight } \leq 60 \mathrm{~kg} \text {, age } \geq 80 \text { years, } \\
\text { serum creatinine } \geq 133 \mu \mathrm{mol} /(1.5 \mathrm{mg} / \mathrm{dL})[\mathrm{or} \text { if } \mathrm{CrCl} 15-29 \mathrm{~mL} / \mathrm{min}]\end{array}$ \\
\hline Dabigatran $^{28}$ & $2 \times 150 \mathrm{mg} / 2 \times 110 \mathrm{mg}$ & No pre-specified dose-reduction criteria $^{a}$ \\
\hline Edoxaban $^{31}$ & $1 \times 60 \mathrm{mg}$ & $\begin{array}{l}1 \times 30 \mathrm{mg} \text { if: weight } \leq 60 \mathrm{~kg}, \mathrm{CrCl} \leq 50 \mathrm{~mL} / \mathrm{min} \text {, concomitant therapy } \\
\text { with strong P-Gp inhibitor (see chapter } \mathbf{5} \text { ) }\end{array}$ \\
\hline Rivaroxaban ${ }^{29}$ & $1 \times 20 \mathrm{mg}$ & $1 \times 15 \mathrm{mg}$ if $\mathrm{CrCl} \leq 50 \mathrm{~mL} / \mathrm{min}$ \\
\hline \multicolumn{3}{|c|}{ Treatment of DVT/PE } \\
\hline & Initial therapy & Remainder of treatment phase \\
\hline Apixaban ${ }^{330}$ & $2 \times 10 \mathrm{mg}, 7$ days & $2 \times 5 \mathrm{mg}$, no dose reduction \\
\hline Dabigatran 331 & Heparin/LMWH & No pre-specified dose-reduction criteria ${ }^{b}$ \\
\hline Edoxaban $^{332}$ & Heparin/LMWH & $1 \times 60 \mathrm{mg}$, same dose reduction as for SPAF (see above) \\
\hline Rivaroxaban 333,334 & $2 \times 15 \mathrm{mg}, 21$ days & $1 \times 20 \mathrm{mg}$, no dose reduction ${ }^{c}$ \\
\hline \multicolumn{3}{|c|}{ Long-term prevention of recurrent DVT/PE (i.e. after 6 months) } \\
\hline & Standard dose & Comments/dose reduction \\
\hline Apixaban 335 & $2 \times 2.5 \mathrm{mg}$ & \\
\hline Dabigatran ${ }^{336}$ & $2 \times 150 \mathrm{mg}$ & No pre-specified dose-reduction criteria ${ }^{d}$ \\
\hline Edoxaban & not specifically studied & \\
\hline Rivaroxaban $^{337}$ & $1 \times 10 \mathrm{mg}$ & e \\
\hline \multicolumn{3}{|c|}{ VTE prevention post-major orthopaedic surgery } \\
\hline & Standard dose & Comments/dose reduction \\
\hline Apixaban 338 & $2 \times 2.5 \mathrm{mg}$ & \\
\hline Dabigatran $^{339,340}$ & $1 \times 220 \mathrm{mg}$ & $f$ \\
\hline Edoxaban $^{341,342}$ & $1 \times 30 \mathrm{mg}$ & Not approved in Europe (only studied in Asia) \\
\hline Rivaroxaban ${ }^{343-346}$ & $1 \times 10 \mathrm{mg}$ & \\
\hline \multicolumn{3}{|c|}{ Stroke prevention post- $\mathrm{PCl}$ (with concomitant atrial fibrillation) ${ }^{\mathrm{g}}$} \\
\hline & Standard dose & Comments/dose reduction \\
\hline Apixaban & \multicolumn{2}{|c|}{ To be determined (pending results of AUGUSTUS trial) } \\
\hline Dabigatran $^{141}$ & $150 \mathrm{mg}$ BID or $110 \mathrm{mg}$ BID & +Clopidogrel or Ticagrelor; no dose reduction \\
\hline Edoxaban & \multicolumn{2}{|c|}{ To be determined (pending results of ENTRUST-AF PCI trial) ${ }^{310}$} \\
\hline Rivaroxaban ${ }^{308}$ & 15 mg OD (+Clopidogrel) & Dose reduction to $10 \mathrm{mg} O D$ if $\mathrm{CrCl} 30-49 \mathrm{~mL} / \mathrm{min}$ \\
\hline
\end{tabular}




\begin{tabular}{|c|c|c|}
\hline \multicolumn{3}{|c|}{ Secondary prevention of atherothrombotic events post-ACS (without AF) } \\
\hline & Standard dose & Comments/dose reduction \\
\hline Rivaroxaban $^{171}$ & $2.5 \mathrm{mg}$ BID & In addition to Aspirin $\pm \mathrm{P}_{2} \mathrm{Y}_{12}$ inhibitor \\
\hline \multicolumn{3}{|c|}{ Secondary prevention of atherothrombotic events in stable CAD (without AF) ${ }^{h}$} \\
\hline & Standard dose & Comments/dose reduction \\
\hline Rivaroxaban ${ }^{347}$ & $2.5 \mathrm{mg} \mathrm{BID}$ & In addition to Aspirin ${ }^{h}$ \\
\hline
\end{tabular}

ACS, acute coronary syndrome; CAD, coronary artery disease.

a SmPC: $2 \times 110 \mathrm{mg}$ if age $\geq 80$ years, concomitant verapamil, increased risk of $\mathrm{Gl}$ bleeding.

'SmPC: $2 \times 110 \mathrm{mg}$ if age $\geq 80$ years, concomitant verapamil, increased risk of Gl bleeding (based on PK/PD analyses; not studied in this setting).

'SmPc: $15 \mathrm{mg}$ if risk of bleeding outweighs risk for recurrent DVT and PE (based on PK/PD analyses; not studied in this setting).

${ }^{d} \mathrm{SmPC}: 2 \times 110 \mathrm{mg}$ if age $\geq 80$ years, concomitant verapamil (both based on PK/PD analyses; not studied in this setting).

eSmPc: $1 \times 20 \mathrm{mg}$ in patients. At high risk of recurrence.

f $\mathrm{SmPc}: 1 \times 150 \mathrm{mg}$ if $\mathrm{CrCl} 30-50 \mathrm{~mL} / \mathrm{min}$; concomitant verapamil, amiodarone, quinidine; age $>75$ years.

${ }^{\mathrm{g}} \mathrm{As}$ outlined in detail in chapter 14, both PIONEER AF-PCl as well as RE-DUAL PCI were powered for safety and were underpowered to determine non-inferiority for indi-

vidual efficacy endpoints.

${ }^{\mathrm{h}}$ As studied in COMPASS; approval of this indication and regimen is pending.

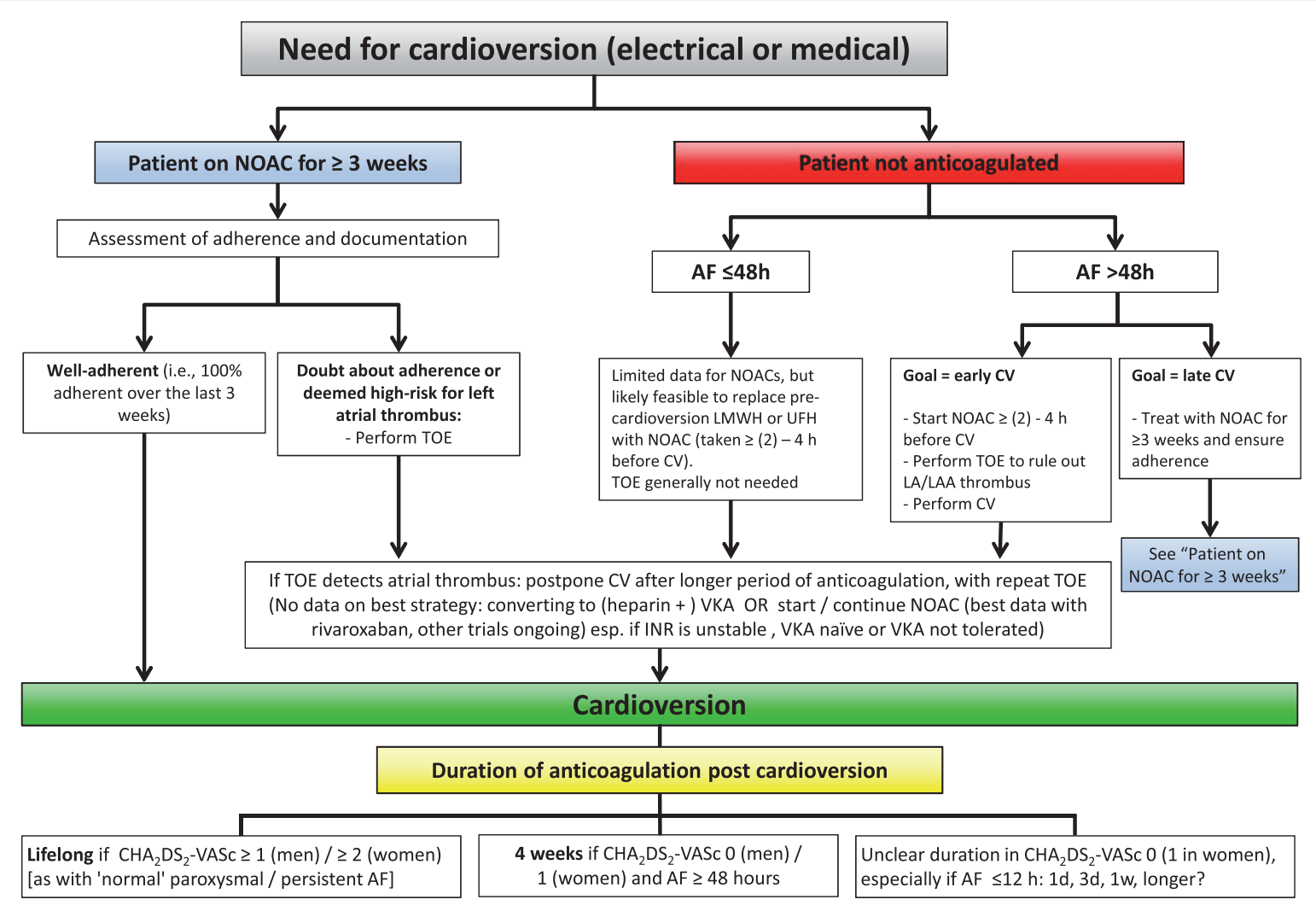

Figure I 2 Cardioversion work-flow in atrial fibrillation patients treated with NOACs, depending on the duration of the arrhythmia and prior anticoagulation. TOE, transoesophageal echocardiography.

bleeding tendency. The $10 \mathrm{mg}$ loading dose is not part of the official labelling (which may change in the near future). Taken together, a strategy with at least a single NOAC dose $\geq 4 \mathrm{~h}$ before cardioversion ( $\geq 2 \mathrm{~h}$ after apixaban loading dose) appears safe and effective in patients with $A F$ of $\geq 48 \mathrm{~h}$ duration, provided that a TOE is performed prior to cardioversion. The alternative is starting anticoagulation with a NOAC for at least 3 weeks followed by cardioversion (without TOE unless high risk patient or deemed non-adherent). 


\section{Cardioverting atrial fibrillation of $\leq 48 \mathrm{~h}$ in an anticoagulation-naive patient}

Even in patients with recent onset $A F$ of $\leq 48 \mathrm{~h}$, different observational studies have shown a lower thromboembolic incidence rate with vs. without anticoagulation, especially in those with a $\mathrm{CHA}_{2} \mathrm{DS}_{2^{-}}$ VASc $\geq 2$ and AF duration $\geq 12 \mathrm{~h}^{355,356}$ Neither $\mathrm{X}$-VeRT nor ENSURE-AF provided information on whether intake of at least one dose of NOAC is a feasible strategy in patients with $A F$ of $\leq 48 \mathrm{~h}$ duration, who are currently often cardioverted after a single dose of LMWH (with continuation of anticoagulation for $\geq 4$ weeks). Some of such patients were included in EMANATE, but publication of the final results is still pending and subgroup results are unknown.

In the absence of data, adherence to current institutional practice with heparin/LMWH with or without TOE may be prudent in such patients. Given the consistent efficacy and safety of NOACs in patients with $\mathrm{AF} \geq 48 \mathrm{~h}$ combined with the similar pharmaco-dynamic and -kinetic properties of NOACs and LMWH, the use of a single dose of NOAC (2) $-4 \mathrm{~h}$ before cardioversion to replace LMWH may be justified in patients with $A F<48 \mathrm{~h}$, without a TOE. Nevertheless in high risk patients (i.e. $\mathrm{CHA}_{2} D S_{2}-\mathrm{VASc} \geq 4$ ) or those in whom there is any doubt about the onset of AF, a TOE strategy or a strategy with longer term anticoagulation (at least for 3 weeks before cardioversion) is recommended. It needs to be kept in mind that the $48 \mathrm{~h}$ cutoff is not binary and cardioversion in the setting of even shorter durations of AF have been associated with an increased risk of stroke, e.g. cardioversion after $12-48 \mathrm{~h}$ vs. $<12 \mathrm{~h}$ ). ${ }^{356,357}$

\section{Duration of anticoagulation post-cardioversion}

The long-term management of patients post-cardioversion depends on the individual patient's $\mathrm{CHA}_{2} \mathrm{DS}_{2}$-VASc score. Men and women with a $C H A_{2} D S_{2}-V A S c \geq 2$ and $\geq 3$, respectively, require long-term anticoagulation independent of the 'success' of cardioversion according to current guidelines. ${ }^{3}$ This is also true for AF with a clear 'trigger' including pulmonary embolism, sepsis, or major surgery, since the trigger does not negate underlying structural or vascular factors associated with increased thromboembolic risk. For AF of $>48 \mathrm{~h}$ duration and a low $\mathrm{CHA}_{2} \mathrm{DS}_{2}$-VASc score ( 0 in men, 1 in women) anticoagulation needs to be continued for 4 weeks post-cardioversion. In contrast, it is currently unknown how long (if at all) the latter patients should be anticoagulated if $A F$ is of shorter duration (especially when $<12 \mathrm{~h}$ ), since $\mathrm{AF}$ and/or cardioversion may contribute to atrial mechanical and/or endothelial dysfunction for hours to days. ${ }^{357}$

\section{Management of a patient with documented left atrial appendage thrombus}

Patients in whom TOE identifies a left atrial thrombus should not undergo cardioversion. Observational and prospective data have not shown a different thrombus incidence in patients treated with NOAC or VKA. ${ }^{349,358-360}$ There are no comprehensive hard clinical endpoint data on the best strategy how to treat a left atrial thrombus with either form of anticoagulant. Previously, standard therapy consisted of VKA therapy with rigorous follow-up and INR monitoring until resolution of the thrombus (with heparin bridging if necessary). Recently, the prospective X-TRA study indicated a thrombus resolution rate of $41.5 \%$ (22/53 patients) with standard dose rivaroxaban $(20 \mathrm{mg} / \mathrm{d})^{361}$ - comparable to the retrospective CLOT-AF registry in which left atrial thrombus resolution was observed in 60/96 patients (62.5\%) in heparin/warfarin treated patients. ${ }^{361}$ Similarly, in the EMANATE trial, thrombus resolution rate was similar in patients treated with apixaban $(52 \%, 12 / 23)$ as with conventional therapy (56\%, 10/18; Ezekowitz et al., ${ }^{8}$ presented at ESC 2017). Individual case reports are equally available for the other NOACs; the RELATED AF study (with dabigatran; NCT02256683) is still ongoing. In congregate, these data indicate that using NOACs for left atrial thrombus resolution may be an option (best data available for rivaroxaban and apixaban), particularly in patients where a VKA is not well tolerated or adequate INR control cannot be obtained.

\section{Atrial fibrillation patients presenting with acute stroke while on non-vitamin $\mathrm{K}$ antagonist oral anticoagulants}

According to controlled clinical trials, the incidence of ischaemic stroke remains $1-2 \%$ per year in patients with AF despite anticoagulant treatment. Adherence to medication needs to be assessed in case of stroke in NOAC treated AF patients. The measurement of anticoagulant plasma level at the time of hospital admission may help to optimize secondary stroke prevention. ${ }^{362}$ In addition, alternative causes of stroke should be assessed in any AF patient.

\section{Management the acute phase of stroke in NOAC treated AF patients}

\section{Patients with acute ischaemic stroke}

According to current guidelines and official labelling, thrombolytic therapy with recombinant tissue plasminogen activator (rt-PA) is approved within $4.5 \mathrm{~h}$ of onset of stroke symptoms but should not be administered in patients on full anticoagulation (e.g. INR $\geq 1.7$ in VKA treated patients) (Figure 13). ${ }^{363}$ Thrombolytic therapy cannot be given within $24 \mathrm{~h}$ after the last intake of a NOAC due to their plasma half-lives (Table 6), which may even be prolonged in renal insufficiency (see chapter 6), the elderly (see chapter 18) and other situations. The case is different for dabigatran due to the availability of the rapid acting specific reversal agent, idarucizumab (see chapter 11). After reversal and assessment of coagulation status, intravenous thrombolysis within $4.5 \mathrm{~h}$ of onset of moderate to severe stroke seems feasible and safe according to case series. ${ }^{364,365}$ In the absence of randomized studies demonstrating the overall efficacy and safety of this approach, balancing the anticipated benefit of this approach vs. its risks is of paramount importance. It remains to be demonstrated whether the same approach will be safe and effective also for Xa-inhibitors once andexanet alpha becomes available.

Published case series suggest that rt-PA may also be safe in patients with low plasma concentrations of NOACs. ${ }^{366,367}$ Despite recent advances reliable and sensitive rapid (point-of-care) tests for the individual NOACs are not widely available yet. ${ }^{362,368,369}$ However, the use of rt-PA may be considered in selected patients on a NOAC in 


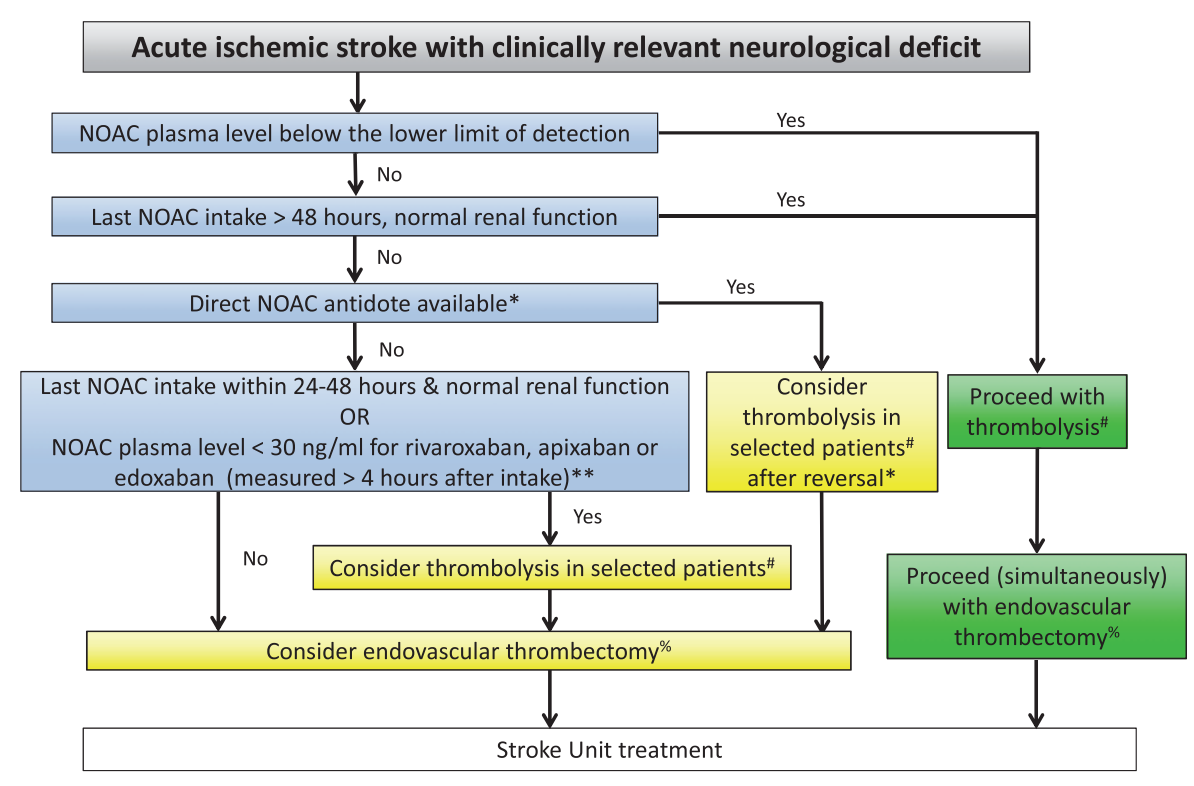

Figure I 3 Acute management of acute ischaemic stroke in a patient on non-vitamin K antagonist oral anticoagulant. *Currently only available for dabigatran (idarucizumab). "Perform systemic thrombolysis only if there are no (other) contraindications for intravenous application of recombinant tissue plasminogen activator according to its label. ${ }^{\%}$ Perform endovascular thrombectomy only if there is a target vessel occlusion and procedure is indicated and feasible according to present evidence. ${ }^{* *}$ According to expert consensus. ${ }^{370}$

cases in which a reliable and NOAC specific coagulation assessment (see chapter 7 ) is available without long delay and demonstrating a concentration $<30 \mathrm{ng} / \mathrm{mL}$ for rivaroxaban, apixaban, or edoxaban (if measured more than $4 \mathrm{~h}$ after drug administration), a reference value which is based on expert consensus only. ${ }^{370}$ Since the efficacy and safety of this strategy needs to be further evaluated in clinical studies, we urge for the implementation of easy-to-use point-of-care testing for the emergency setting. In contrast, the use of thrombolysis in situations with uncertainty about the anticoagulation status (e.g. in AF patients with aphasia, unknown time of last NOAC dose, and lack of availability of rapid assessment of plasma levels) cannot be recommended.

There is a proven benefit of endovascular thrombectomy up to $7.3 \mathrm{~h}$ after stroke onset in selected non-anticoagulated patients with a distal occlusion of the internal carotid artery or the proximal middle cerebral artery. ${ }^{371}$ Interestingly, endovascular thrombectomy also seems to be beneficial in highly selected stroke patients with a distal occlusion of the internal carotid artery or the proximal middle cerebral artery and favourable perfusion mismatch (according to the DEFUSE or DAWN study) within 6 to up to $24 \mathrm{~h}$ of last seen normal. $^{372,373}$ The European Stroke Organization recommendations now mention the use of endovascular thrombectomy as 'first-line treatment' in patients with contraindication for intravenous thrombolysis, while the AHA's guidelines provide no specific recommendation in this regard. ${ }^{363,374}$ Although the trials underlying these recommendations either excluded or contained just a few patients on VKA or NOAC, the small amount of data available suggests that endovascular thrombectomy may be safe also in these individuals. Of note, the potential impact of present anticoagulation on reperfusionrelated bleeding risk has to be taken into account and a comparably high rate of asymptomatic haemorrhagic transformation was observed in a prospective registry including 28 NOAC patients undergoing mechanical recanalization. ${ }^{375}$ Further prospective data are urgently needed.

\section{Patients with acute intracranial bleeding}

About two thirds of all NOAC-related intracranial bleedings (ICBs) are intracerebral and about one third of all ICBs are subdural bleedings. ${ }^{376,377}$ According to a meta-analysis of retro- as well as prospective studies, patients with intracerebral bleeds on NOAC (without using idarucizumab as a specific reversal agent of dabigatran) had the same poor prognosis as patients on VKA, ${ }^{378}$ while a more recent and much larger retrospective analysis of the Get With the GuidelinesStroke program found a more favourable outcome with NOACs compared with VKA. ${ }^{379}$ A neurologist/stroke physician should examine all patients presenting with ICB on a NOAC, and neurosurgical consult should be solicited.

Recommendations for the treatment of ICB under oral anticoagulants are published, but the available level of evidence is low for NOAC-related ICB. In analogy to patients with acute ICB being treated with warfarin, discontinuation of the drug, urgent blood pressure management and rapid correction of the coagulation status (see also chapter 11) is needed to limit haematoma enlargement in patients under NOAC. ${ }^{376,380,381}$ Whether the use of PCC is helpful 
in NOAC-related ICB is a matter of debate since a retrospective multicentre analysis did not prove a significant benefit on haematoma enlargement. ${ }^{382}$ For dabigatran related ICB reversal is possible via infusion of idarucizumab (see chapter 11). According to a reported case series, ${ }^{365}$ haematoma growth was observed in two out of twelve ICB patients treated with dabigatran receiving idarucizumab on hospital admission. Despite present recommendations, the efficacy of this reversal strategy is unclear and needs to be further evaluated in clinical studies.

\section{Management in the post-acute phase \\ Atrial fibrillation patients post-ischaemic stroke}

There is no evidence from RCTs to prefer one NOAC over the other or to switch from one NOAC to another in patients with a history of ischaemic stroke under NOAC therapy (Figure 14). Appropriate dosing as well as patient specific issues need to be assessed. 41,93,202 Substantial study data regarding timing of reinstitution of oral anticoagulation by using a NOAC after transient ischaemic attack (TIA) or stroke in AF patients are missing, ${ }^{383}$ as Phase III trials excluded patients within 7-30 days after stroke.

Therefore, present recommendations are based on consensus opinion, and NOACs should be (re-) initiated in analogy to clinical practice with VKAs. Recommendations on (re-) starting of oral anticoagulation after ischaemic stroke must outweigh (recurrent) stroke risk vs. secondary haemorrhagic transformation (Figure 14). ${ }^{3,383}$ As stated in the current ESC guidelines, ${ }^{3}$ oral anticoagulation using a NOAC may be continued (according to prescription and label) or started one day after a transient ischaemic attack (TIA) and exclusion of ICB by imaging. If stroke size is not expected to substantially increase the risk of secondary haemorrhagic transformation in patients with mild stroke, oral anticoagulation may be initiated $\geq 3$ days after an ischaemic stroke. In patients with moderate stroke, anticoagulation may be started $\geq 6-8$ days and in patients with severe stroke at $\geq 12-14$ days, after excluding secondary haemorrhagic transformation by repeating brain imaging [using computed tomography (CT) or magnetic resonance imaging (MRI)]. ${ }^{383-385}$

Due to the rapid onset of action of NOACs as well as an associated risk of bleeding, 'bridging' with heparin (LMWH or UFH) is not recommended. Moreover, a meta-analysis revealed that administration of parental anticoagulants within 7-14 days after ischaemic stroke is associated with a significant increase in symptomatic ICB. ${ }^{386}$

\section{Atrial fibrillation patients with ischaemic stroke and concomitant atherosclerosis}

Besides a (well-tolerated) statin therapy, temporally limited addition of aspirin to a NOAC may be considered in selected patients if underlying large-vessel disease is suspected and bleeding risk is considered to be comparably low. However, evidence for both approaches is lacking and further studies are required. Patients with $\mathrm{AF}$ and known carotid atherosclerosis with an asymptomatic stenosis of the internal carotid artery should be treated with a statin and an oral anticoagulant, without the need for additional antiplatelet therapy, similar to the situation in stable coronary heart disease (see chapter 14). Acute stroke patients with AF and 'symptomatic' highgrade carotid stenosis should preferably undergo carotid endarterectomy, ${ }^{387}$ as carotid stenting would result in the need for dual antiplatelet therapy in addition to anticoagulation therapy with a subsequently higher risk of major bleeding. In patients undergoing endarterectomy, aspirin is recommended prior to and for some days after surgery. Aspirin should be stopped after (re-) starting oral anticoagulation.

\section{Patients post intracranial bleeding}

Apart from its immediate prognosis, an ICB in the setting of AF is also associated with later ischaemic stroke and mortality, partly due to the cessation of anticoagulation after ICB (Figure 15). ${ }^{388-390}$ Evidencebased guidelines regarding the use of NOACs in AF patients after ICB are not available. A history of a spontaneous ICB constitutes a contraindication against anticoagulation according to labelling of VKAs and NOACs, unless the cause of the bleeding (like uncontrolled hypertension, aneurysm or arteriovenous malformation, or medical 'triple' therapy) has been reversed. ${ }^{3}$ A recent meta-analysis of observational studies demonstrates that restarting VKA (but not antiplatelet agents) is associated with a significantly lower rate of ischaemic stroke without significantly increasing the risk of recurrent ICB. ${ }^{389}$ However, publication bias as well as selection bias have to be taken into account. In the absence of RCTs, a case-by-case consideration is needed whether or not to reintroduce anticoagulation of any type in patients who have experienced an anticoagulation-related ICB (Figure 15). ${ }^{3}$ Adequate blood pressure control is of paramount importance in all patients post ICB. ${ }^{380}$ Left atrial appendage occlusion may be considered as potential substitute for long-term anticoagulation in AF patients postICB. ${ }^{3}$ However, this strategy requires a period of antiplatelet treatment post-deployment, which also carries a risk of ICB. The safety and effectiveness of shorter duration antiplatelet therapy (or foregoing anticoagulation altogether) is not known. Overall, RCT evidence for LAA occlusion after OAC-related ICB under OAC is missing, which is why, ideally, treatment should occur in the framework of a randomized trial to contribute to evidence.

\section{Patients post intracerebral bleeding}

In analogy to the management of VKA-related intracerebral bleeding, administration of NOACs may be restarted 4-8 weeks after intracerebral bleeding if the individual risk of cardioembolic stroke is estimated to be high and the risk of recurrent ICB is estimated to be lower. ${ }^{391}$ In practice, however, the same risk factors (including old age, hypertension, and previous stroke) are predictive for ischaemic stroke as well as recurrent intracerebral bleeding. ${ }^{381}$

Arguments for not resuming or initiating anticoagulation in intracerebral bleeding patients with AF should be assessed on an individual basis (Figure 15). ${ }^{3,380}$ Patients with (probable) cerebral amyloid angiopathy have a very high risk of recurrent ICB and should not be anticoagulated. ${ }^{390}$ Whether long-term anticoagulation should be avoided after a lobar bleed, as currently recommended by the AHA guidelines, is a matter of debate, since a recent meta-analysis of three retrospective studies indicate decreased mortality and favourable functional outcome after resumption of oral anticoagulation after intracerebral bleeding, irrespective of haematoma localization. ${ }^{391}$

\section{Patients post subarachnoid haemorrhage}

There is little evidence to guide the resumption of OAC treatment in patients with AF following subarachnoid haemorrhage. A thorough 


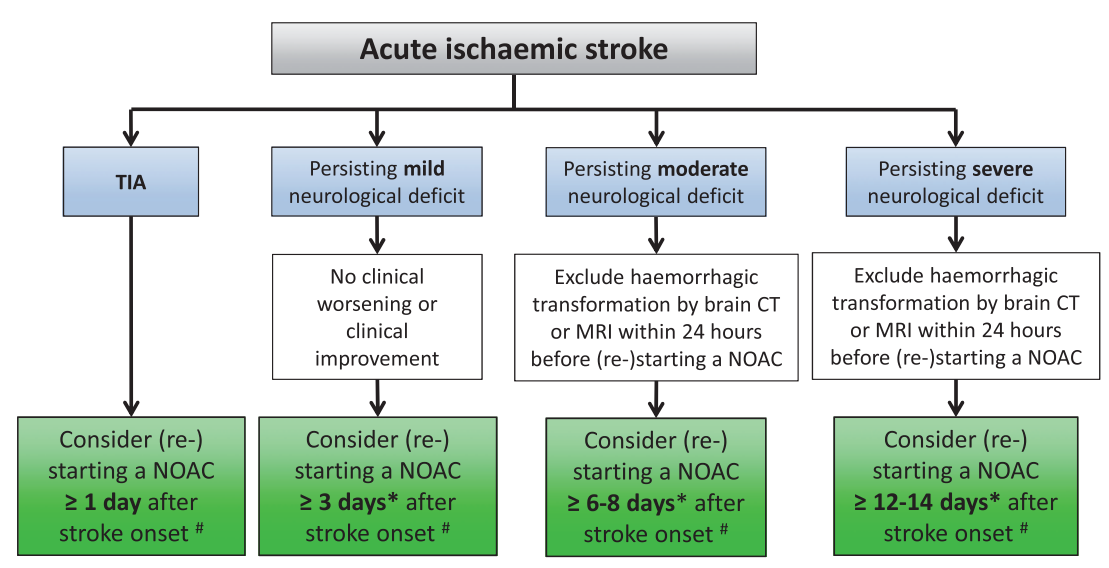

Figure I4 (Re-) initiation of anticoagulation after transient ischaemic attack/stroke. (Re-) start only in the absence of contraindications and if stroke size is not expected to substantially increase the risk of secondary haemorrhagic transformation. *Consider shorter delays to (re-) start a non-vitamin $\mathrm{K}$ antagonist oral anticoagulant if there is a very high risk of stroke recurrence (e.g. left atrial appendage thrombus) and no haemorrhagic transformation on follow-up brain imaging (using computed tomography or magnetic resonance imaging). Consider longer delays to (re-)start a nonvitamin $\mathrm{K}$ antagonist oral anticoagulant according to the recommendations made in the European Society of Cardiology Atrial Fibrillation Guidelines 2016. "Without proven evidence; consider inclusion of patient in an ongoing trial.

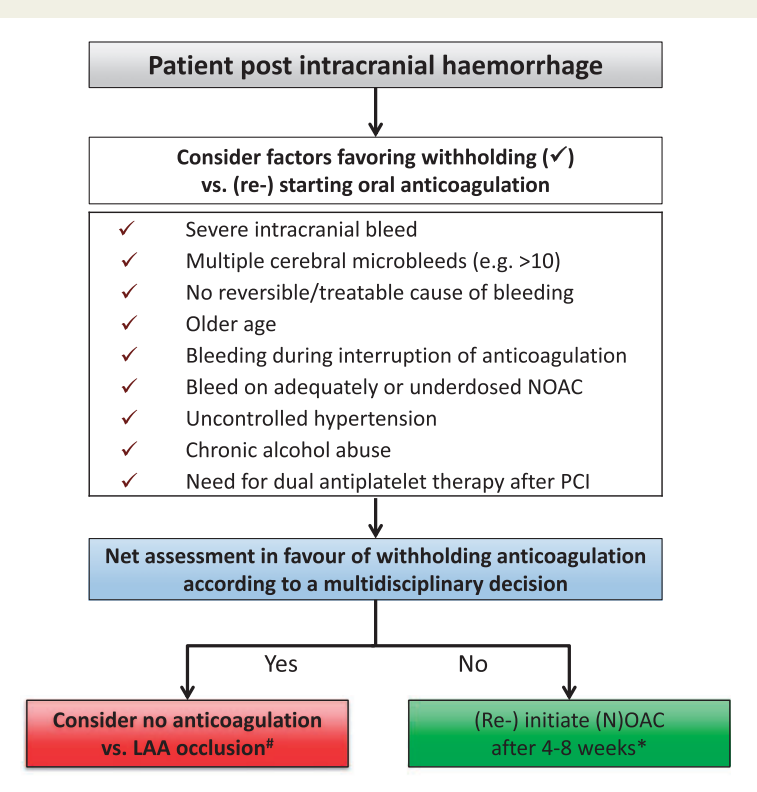

Figure 15 (Re-) initiation of anticoagulation post intracranial bleeding. "Without evidence; ideally include the patient in an ongoing trial. *Brain imaging (CT/MRI) should be considered before (re-)initiation of (non)-vitamin K antagonist oral anticoagulant.

angiographic evaluation and treatment of underlying aneurysm or arteriovenous malformation is needed. Moreover, neurological/neurosurgical evaluation regarding future risk of re-bleeding is key to balance the risk vs. benefit of OAC resumption in such cases. When subarachnoid haemorrhage occurs in AF patients taking a NOAC in the absence of a remediable aetiology it seems prudent not to reinitiate OAC treatment. Despite the absence of data, LAA closure should be considered, ideally in the framework of a randomized trial.

\section{Patients post epidural or subdural haematoma}

Although there are no specific data, it appears to be safe to start or reinitiate anticoagulation about 4 weeks after (surgical removal of) traumatic epidural or subdural haematoma, if ongoing (chronic) alcohol abuse or a substantial risk of falling is not present (see chapter 18). Adequately dosed NOAC or no anticoagulation at the time of non-traumatic epidural or subdural haematoma does not support (re-) initiation of oral anticoagulation. ${ }^{3}$ According to clinical presentation and haematoma extension, brain imaging (using CT or MRI) is recommended before (re-) starting OAC.

\section{NOACs in special situations}

\subsection{Non-vitamin $K$ antagonist oral anticoagulants in the frail and older patients}

The $\geq 75$-year-old patient

The incidence of AF rises steadily with each decade. ${ }^{392,393}$ Stroke prevention in older AF patients is important as stroke risk rises dramatically with age. ${ }^{394}$ However, OAC remains underutilized in older age groups. ${ }^{395}$ Older people with AF do better on OAC than not and on NOACs rather than VKA. ${ }^{396-398}$

All trials of NOAC treatment in AF included significant populations of older people (defined as $\geq 75$ years) ranging from $31 \%$ to $43 \%$ in the individual trials, comprising over 27000 older patients in whom 
NOACs have been studied. Meta-analyses of NOAC trial data suggest no interaction of age for safety and efficacy. ${ }^{246}$ Importantly, the higher absolute risk resulted in a larger absolute risk reduction by using NOACs instead of VKA in these older patients, resulting in a lower number needed to treat compared to younger patients. ${ }^{399}$ Older patients had more bleeding but the overall pattern of bleeding observed (reduced intracranial and increased Gl bleeding) showed no difference between NOACs and VKA. ${ }^{246}$ While ICB remains lower with all NOACS compared with VKA, individual trial results showed heterogeneity on the interaction between age and bleeding outcomes. There was a significant interaction between age and increased extracranial major bleeding with both doses of dabigatran. ${ }^{155}$ Conversely, no significant age interaction on rates of extracranial major bleeding was seen with apixaban, edoxaban, or rivaroxaban compared with overall trial results. ${ }^{399-401}$ Importantly, certain comorbidities (renal insufficiency in particular, see chapter 6) are more common in the older patient, and the individual choice of the NOAC needs to take this into consideration. One interesting study investigating low-dose edoxaban in the management of elderly Japanese patients with atrial fibrillation who are ineligible for standard oral anticoagulant therapies (ELDERCARE-AF study) is currently ongoing. ${ }^{402}$

\section{Frailty and falls}

Frailty and pre-frail states are common with age and raise specific considerations with regard to the risk-benefit ratio of OAC. Frailty is commonly defined as a rules-based distinct phenotype or by clinical judgement of deficits in function in a frailty scale (see Table 14). ${ }^{403-405}$

Among others, frailty is a risk for rapid deterioration of renal function (see chapter $\mathbf{6}$ ) and risk of falling. Community dwelling individuals over 65 years have a $1-2 \%$ risk of falling per year; only $5 \%$ of falls, however, result in fracture and hospitalization. ${ }^{406}$ Falls and risk of subdural haemorrhage in particular are often considered by physicians as a contraindication to $\mathrm{OAC}{ }^{407}$ While in states of severe frailty with poor physical functioning and limited life expectancy there may be limited benefit to OAC, a Markov decision analytic model has demonstrated that with VKA a patient would have to fall 295 times in order for the risk of a subdural haematoma to outweigh the benefit of anticoagulation. ${ }^{408}$ Given the even lower risk of subdural bleeding compared with VKA, this 'number needed to fall' would be even higher with the use of NOACs.

The risk of falling can be estimated using simple or more sophisticated tools (Table 15). The effect of NOACs vs VKA in patients at risk of falling was specifically analysed in two NOAC trials (prospectively defined in ENGAGE-AF TIMI 48, retrospectively in ARISTOTLE). ${ }^{52,409}$ The treatment effect of the respective NOAC was consistent in patients at increased vs. not at increased risk of falling. However, the larger absolute risk of events of patients at increased risk of falling resulted in a larger absolute risk reduction vs. VKA and, consequently, a lower number needed to treat compared to those not at an increased risk of falling.

In summary, frailty per se should not be an exclusion criterion to anticoagulate since frail and older patients are at an increased risk of stroke and have been shown to benefit from OAC. The benefit of NOACs over VKA has best been demonstrated for edoxaban and apixaban in this patient population. To improve things further, all falling patients on OAC should be referred to a falls service for multidisciplinary assessment of diagnosis, risk and to address remediable pathology and/or prescribe interventions (e.g. exercise programs; home environmental assessment etc.) that reduce risk of further falls. ${ }^{411-413}$

\section{Dementia and anticoagulation}

Dementia is common in older age groups. A stroke is a very significant event for patients with dementia with a greater risk of cognitive and functional decline, loss of independence and institutionalization compared to non-dementia patients. ${ }^{414}$ Indeed, atrial fibrillation is itself a risk factor for dementia and there is encouraging evidence that use of OAC may reduce the risk of dementia in AF patients. ${ }^{415,416}$

Dementia does pose unique considerations, however, when considering anticoagulation and in particular around patient capacity in decision making, choice of treatment and managing drug adherence safely. Importantly dementia should not be a viewed as a general contraindication to anticoagulation, especially if well managed from a logistical point of view (see below). All patients with dementia should have a careful assessment of their ability to understand and make a treatment decision regarding $O A C$ in $A F$, with indicative risks of stroke and bleeding provided. Where capacity is lacking, it may be reasonable for the physician to recommend treatment on the basis of the 'best medical interest' principle, ideally including next of kin assent.

Adherence to OAC intake is a significant consideration in dementia. Once daily medications, weekly tablet boxes, reminders or blister packing may be helpful. Paradoxically, the fact that others take care of providing medication to dementia patients may guarantee higher adherence. The possible advantages of electronic monitoring, or even telemonitoring, in this population should further be explored. ${ }^{51}$

\subsection{Obesity and low body weight}

\section{Obesity}

The WHO defines overweight and obesity as a body mass index (BMI) of greater than 25 and $30 \mathrm{~kg} / \mathrm{m}^{2}$, respectively. The incidence of obesity has tripled since 1975. In 2016, 650 million adults (13.1\% worldwide population) were obese. ${ }^{417}$ Among many other things, obesity also increases the risk of atrial fibrillation and recurrences of atrial fibrillation after successful ablation. ${ }^{418-420}$. As such, weight loss is an integral part in the multidisciplinary treatment of patients with AF and obesity. ${ }^{421}$

Obesity affects the pharmacokinetics of drugs, including the volume of distribution (of lipophilic drugs in particular) as well as drug clearance. Indeed, renal blood flow and $\mathrm{CrCl}$ have been shown to be increased in obesity and could increase elimination of OACs. ${ }^{422}$ A number of studies of VKA have indicated that obese patients require greater doses and longer lead-in periods for achieving therapeutic INR values. ${ }^{423}$

Studies of dabigatran reported no effect of weight on pharmacokinetic variables although analysis in older healthy individuals did not include very obese patients. ${ }^{159,166,182}$ Pharmacokinetic data on both rivaroxaban and apixaban initially reported weight-dependent changes on volume distribution and half-life across a range of weights; however, these were felt unlikely to be clinically significant. ${ }^{185,424,425}$ Data with edoxaban suggests low body weight may be a factor in 


\section{Table I4 The 'Canadian Study of Health and Aging' (CHSA) Clinical Frailty Scale}

From http://www.csha.ca and Ref. ${ }^{404}$

(1) Very fit - People who are robust, active, energetic, and motivated. These people commonly exercise regularly. They are among the fittest for their age.

(2) Well - People who have no active disease symptoms but are less fit than category 1. Often, they exercise or are very active occasionally, e.g. seasonally.

(3) Managing well - People whose medical problems are well controlled, but are not regularly active beyond routine walking.

(4) Vulnerable - While not dependent on others for daily help, often symptoms limit activities. A common complaint is being 'slowed up', and/or being tired during the day.

(5) Mildly frail - These people often have more evident slowing, and need help in high order IADLs (finances, transportation, heavy housework, medications). Typically, mild frailty progressively impairs shopping and walking outside alone, meal preparation and housework.

(6) Moderately frail - People need help with all outside activities and with keeping house. Inside, they often have problems with stairs and need help with bathing and might need minimal assistance (cuing, standby) with dressing.

(7) Severely frail - Completely dependent for personal care, from whatever cause (physical or cognitive). Even so, they seem stable and not at high risk of dying (within $\sim 6$ months).

(8) Very severely frail - Completely dependent, approaching the end of life. Typically, they could not recover even from a minor illness.

(9) Terminally ill - Approaching the end of life. This category applies to people with a life expectancy $<6$ months, who are not otherwise evidently frail.

reduced clearance of the drug, ${ }^{426}$ and a converse situation would seem plausible.

Concerns have been expressed about the reliability of the anticoagulant effect of NOACS in obese patients. ${ }^{427,428}$ Weight was not an exclusion criterion in any of the NOAC trials in AF or VTE. However, case reports of treatment failure with low serum levels of dabigatran have been reported in cases of severe obesity (BMI $\left.\geq 40 \mathrm{~kg} / \mathrm{m}^{2}\right) .{ }^{429,430}$

Apixaban demonstrated no difference in efficacy and safety in patients $<60 \mathrm{~kg}$ vs. $>60 \mathrm{~kg},{ }^{30}$ but patients with a BMI $\geq 30 \mathrm{~kg} / \mathrm{m}^{2}$ had a trend towards a better outcome compared to the remainder of the study (independent of treatment). ${ }^{431}$ This was in contrast to the reduced bleeding seen in the obese patient group in the AMPLIFY study of apixaban in the treatment of VTE. ${ }^{330}$ Similarly in ROCKET$A F$, obese patients $\left(B M I \geq 35 \mathrm{~kg} / \mathrm{m}^{2}\right.$ ) had a reduced stroke risk compared with the remainder of the cohort, and there was no interaction for the efficacy and safety of rivaroxaban vs. warfarin depending on BMI. $^{432}$ ENGAGE-AF did not (yet) report a sub-analysis of efficacy and safety with edoxaban according to weight criteria. ${ }^{31}$ Clinical trial data from use of edoxaban in acute VTE, included 611 (14.1\%) patients $>100 \mathrm{~kg}$ and sub analysis by weight showed no difference in safety or efficacy. ${ }^{332}$

Because of limited data in extreme obesity, the use of VKA in patients with a $\mathrm{BMI} \geq 40 \mathrm{~kg} / \mathrm{m}^{2}$ or weight $>120 \mathrm{~kg}$ should be considered (in line with recommendations from the International Society on Thrombosis and Haemostasis). ${ }^{427}$ In rare case when a NOAC is needed in such circumstances, specific measurements of drug trough levels should be considered. This, however, should only be done under the guidance of a haematologist and in the knowledge that hard clinical outcome data do not exist for such an approach.

\section{Low body weight}

There is no unifying definition of low body weight and future criteria may need to be race specific as Asian populations tend to be smaller and leaner. Low body weight may increase exposure to any NOAC and as such increase the risk of bleeding. ${ }^{433}$ Importantly, patients with low body weight frequently present with other conditions and co-morbidities which may increase the risk of stroke as well as bleeding, including old age, frailty, cancer, and renal insufficiency. Of note, renal function may be overestimated in underweight patients due to their reduced muscle mass (especially when calculated with the MDRD formula; see chapter 6). As such, special care is needed when anticoagulating these patients.

Body weight $\leq 60 \mathrm{~kg}$ was a dose-reduction criterion for apixaban (if also age $\geq 80$ years and/or creatinine $\geq 1.5 \mathrm{mg} / \mathrm{dL}$, see chapter 15 ) as well as for edoxaban. For these drugs, efficacy and safety compared to warfarin was consistent in the (few) underweight patients when compared with the remainder of the study cohort. ${ }^{30,31}$ As such, both drugs may be a preferred choice for patients $<60 \mathrm{~kg}$.

Dabigatran was studied post hoc in patients with low body weight $(<50 \mathrm{~kg}$ ) with consistent efficacy and safety compared with the remainder of the study cohort. ${ }^{28}$ However, observational studies have suggested that low BMI $\left(<23.9 \mathrm{~kg} / \mathrm{m}^{2}\right)$ can be an independent predictor of bleeding events with dabigatran. ${ }^{434}$ In addition, frequently co-existing renal insufficiency may make dabigatran a less preferably option for the underweight patients. Also rivaroxaban showed similar efficacy and safety in an exploratory analysis of lower body weight, but only patients $<70 \mathrm{~kg}$ were compared with those $>70 \mathrm{~kg} .{ }^{29}$ No outcome data are available for patients with $<60 \mathrm{~kg}$ or $<50 \mathrm{~kg}$ in patients on the full AF dose of rivaroxaban. 


\section{Table I 5 Examples of falls risk tools}

\begin{tabular}{|l|}
\hline (A) High risk of falls (from ENGAGE-AF TIMI 48) ${ }^{52}$ \\
\hline Presence of one or more of \\
- Prior history of falls \\
- Lower extremity weakness \\
- Poor balance \\
- Ornitive impairment \\
- Use of psychotropic drugs \\
- Severe arthritis \\
\hline
\end{tabular}

\begin{tabular}{|l|l|}
\hline \multicolumn{2}{|l|}{ (B) Probability falls assessment ${ }^{410}$} \\
\hline 1 point for each 'Yes' & Yes/No \\
\hline Previous falls & \\
\hline Medications & Yes/No \\
\hline$>4$ & Yes/No \\
\hline Psychotropics & Yes/No \\
\hline Low visual acuity & Yes/No \\
\hline Diminished sensation & Yes/No \\
\hline Near tandem stand 10s & Yes/No \\
\hline Alternate step test 10s & Yes/No \\
\hline Sit to stand 12s & \\
\hline
\end{tabular}

\begin{tabular}{|l|l|l|l|l|}
\hline Score & $0-1$ & $2-3$ & $4-5$ & $6+$ \\
\hline Probability of fall per year & $7 \%$ & $13 \%$ & $27 \%$ & $49 \%$ \\
\hline
\end{tabular}

Severely underweight patients $(<50 \mathrm{~kg})$ were clearly underrepresented in the large outcome trials. As such, even for NOACs that were dose-reduced based on body weight (apixaban and edoxaban), data are limited for these patients. Of note, bleeding may also be increased with VKA therapy in underweight patients. ${ }^{431}$ If therapy with a NOAC is warranted in these individuals, measurement of trough levels may be considered to check for accumulation of the drug. ${ }^{435}$ However, no evidence-based recommendations can be given regarding (further) dose reduction in such cases.

\subsection{Women of reproductive age}

All OAC use should be considered with caution in women of childbearing age and an appropriate test to rule out pregnancy and contraceptive counselling advice arranged before initiation of any agent. Abnormal uterine bleeding (AUB; formerly called menorrhagia), occurs in $9-14 \%$ of the general female population of reproductive age, ${ }^{436}$ which may be exacerbated by oral anticoagulants. ${ }^{437}$
In a recent case series of NOAC use in the treatment of acute VTE in women of reproductive age, rivaroxaban was associated with prolonged (>8 days) menstrual bleeding ( $27 \%$ vs. $8.3 \%, P=0.017$ ), increased need for menorrhagia-related medical or surgical intervention ( $25 \%$ vs. $7.7 \%, P=0.032)$, and more adaptations of anticoagulant therapy ( $15 \%$ vs. $1.9 \%, P=0.031)$ compared with VKA. ${ }^{438} \mathrm{~A}$ similar trend towards increased AUB with rivaroxaban compared to enoxaparin has also been reported. ${ }^{439}$ Registry data report a $32 \%$ incidence of AUB in women of reproductive age $(n=178)$ on factor Xa inhibitor. ${ }^{40}$ Most cases were managed successfully with change of hormonal or anticoagulation therapy, including temporary discontinuation or cessation of factor $\mathrm{Xa}$ inhibitor medication. Some authors have expressed concern about the lack of robust data for NOAC use in this population with $A F .^{441}$ In any case, women should be counselled about the risk of increased menstrual bleeding while on NOAC and monitored carefully especially during the first cycles after NOAC initiation. $^{442}$

All cases of AUB on OAC need to have gynaecological assessment for underlying structural problems and possibility of local hormonal treatments and/or surgical procedure to reduce risk of recurrence of AUB. Importantly, NOACs are contraindicated in pregnancy as well as during breastfeeding.

\subsection{Non-vitamin $K$ antagonist oral anticoagulants in Athletes}

$\mathrm{AF}$ is the most common arrhythmia in athletes and endurance athletes are known to be more prone to $A F^{443-446}$ Additional risk factors for stroke may be uncommon in this population; however, older individuals are increasingly engaged in competitive and/or vigorous sports activities. ${ }^{447}$

If the $\mathrm{CHA}_{2} \mathrm{DS}_{2}$-VASc score is $\geq 1$ in men and $\geq 2$ in women, the use of anticoagulation may be warranted in such settings according to current guidelines. ${ }^{3}$ Traditional advice to athletes on OAC for VTE has been to avoid contact sports while on treatment and there is little published evidence on the use of NOACs in AF in such populations. The use of a OD agent may be preferable with intake in the evening to avoid high levels of the drugs during the actual exercise, but no outcome data are available to support this. All athletes presenting with AF should have a full cardiological assessment.

\subsection{Epilepsy}

A risk of seizures has been reported in $>5 \%$ of overall post-stroke patients. ${ }^{448,449}$ Following an unprovoked seizure after stroke, the risk of subsequent unprovoked seizures is about $65 \%$ within 10 years. ${ }^{450}$

OAC poses a special risk for patients with epilepsy due to the risk of injury during a seizure (with or without falling). Most seizures in older people or post-stroke patients are focal in onset. However, patients who do suffer rare generalized atonic seizures are particularly vulnerable to head trauma while tongue biting is a risk in the tonic component of generalized seizures.

Anticoagulation is affected by antiepileptic drugs via various potential interactions (Table 5). ${ }^{147}$ A number of antiepileptic drugs can in addition cause thrombocytopenia or platelet dysfunction. ${ }^{147}$ The significance of these drug-drug interactions is still largely unknown with only occasional case reports available. In 
some situations of severe, relevant interactions NOACs may not be the preferred choice.

\section{Anticoagulation in atrial fibrillation patients with a malignancy}

\section{The scope of the problem}

Cancers are not infrequent in older patients, similar to AF. One study found a prevalence of $2.4 \%$ of pre-existing $\mathrm{AF}$ and $1.8 \%$ new $\mathrm{AF}$ among cancer patients. ${ }^{451}$ Cancer and cancer therapy may in turn precipitate $A F$, while both age and malignancy are independent risk factors for thrombosis and bleeding.

The greater incidence and prevalence of $\mathrm{AF}$ in patients with malignancy may result from the presence of comorbid conditions (e.g. hypertension, heart failure), a direct tumour effect (including dehydration, altered sympathetic tone due to anxiety or pain, systemic inflammation, etc.) or as a complication of cancer therapy (e.g. after lung cancer surgery or as a side effect of specific targeted therapies such as tyrosine kinase inhibitor ibrutinib). ${ }^{452-455}$ The increasing survival of cancer patients may additionally increase the incidence of AF among patients with active and past malignancies.

The risk of VTE is increased in the presence of cancer through a host of possible mechanisms. ${ }^{456}$ Brain, pancreatic, ovarian, lung, or haematological malignancies, as well as many cancer treatments (e.g. cisplatin, gemcitabine, 5-fluorouracil, erythropoietin, granulocyte colony stimulating factors) are associated with particularly increased thromboembolic risk. ${ }^{457}$

Conversely, cancers may cause infiltrative liver failure resulting in thrombocytopenia or coagulopathy and increased risk of bleeding. Tumours may erode into blood vessels directly, and many $\mathrm{Gl}$ and solid tumours such as intracranial tumours, renal cell carcinoma, or metastatic melanoma are very vascular and prone to bleeding. Haematologic malignancies may cause coagulation defects thus increasing the risk of bleeding further. In addition, every form of cancer therapy, be it surgery, radiation, or chemotherapy, may induce bleeding through local wounds (surgery), tissue damage (radiation), or systemic antiproliferative effects reducing platelet count and function (e.g. chemotherapy, some forms of irradiation).

\section{Anticoagulant therapy in atrial fibrillation patients with malignancy}

So far, the only published RCT specifically targeting cancer patients stems from the HOKUSAI-VTE Cancer trial comparing edoxaban with LMWH in patients with VTE (but not AF). ${ }^{458}$ Edoxaban proved to be non-inferior regarding the primary endpoint of recurrent VTE and major bleeding; while recurrent VTE trended to be lower with edoxaban, major bleeding was higher (driven by an increased risk of upper Gl bleeding in patients with gastrointestinal cancer). In line with these findings, several meta-analyses of the small subgroup of cancer patients in VTE trials reported similar or better efficacy of NOACs in comparison to VKA or LMWH for VTE prevention, although major bleeding rates were higher. ${ }^{459,460}$ Most of these cancer patients may have been clinically stable, in contrast to those requiring active therapy or in a palliative setting.
Table 16 Atrial fibrillation and malignancy

\section{Interdisciplinary teamwork}

(1) Estimate individual patient risk profile

- AF-related risk factors ( $\mathrm{CHA}_{2} \mathrm{DS}_{2}-\mathrm{VASc}$, bleeding risk)

- Cancer-related risk factors (type, liver metastases, coagulopathy, renal/hepatic function etc.

- Treatment-related risk factors (thrombocytopenia, surgery, radiation, central lines etc.)

(2) Choose anticoagulant

- Current standard of care: VKA/(LMWH) ${ }^{a}$

- NOACs: Available data scarce, but encouraging

- Consider patient preference (VKA vs. NOAC)

(3) Protect the patient

- Gastric protection (PPI/H2 blockers)

- Beware of drug-drug interactions (Table 4)

- Dose reduction/treatment interruption (if platelets $<50 k$, renal dysfunction, bleeding, ...)

Beware

- Risk of thromboembolism $\uparrow$

- Risk of bleeding $\uparrow$

${ }^{a}$ If oral therapy is not possible reversion to LMWH is reasonable.

Moreover, in how far these findings apply to AF patients with cancer requires further data. In cancer patients who develop incident $A F$, VKAs, or LMWH have been traditionally preferred over NOACs, based on greater clinical experience with these drugs, possibility for closer monitoring and availability of 'reversal' options. However, evidence for stroke prevention with LMWH in AF is lacking and LMWH is contraindicated in secondary prevention in the setting of acute stroke. $^{386}$ Active malignancy was an exclusion criterion in most NOAC AF trials, and although there were a few patients with cancer in the Phase III AF trials, the absence of information on the type and stage of cancer precluded any relevant subgroup analysis. An exploratory analysis of AF patients with active cancer $(n=157)$ or a history of malignancy $(n=1079)$ in the ARISTOTLE trial showed consistently superior efficacy and safety of apixaban vs. warfarin in patients with and without cancer. ${ }^{461} \mathrm{~A}$ large registry using prescription based analysis for AF patients on VKA or NOAC with and without cancer recently reported equivalence for bleeding and thromboembolic risk and cancer status, although the rates of both were lower in the NOAC population. ${ }^{462}$ However, much is still unknown about drugdrug interactions between NOACs and specific chemotherapeutic agents, urging further caution (Table 4). ${ }^{144}$

Overall, antithrombotic therapy in patients with AF suffering from a malignancy needs a dedicated interdisciplinary team approach (Table 16). ${ }^{463}$ Especially, when myelosuppressive chemotherapy or radiation therapy is planned, temporary dose reduction or cessation of NOAC therapy needs to be evaluated, taking into account full blood counts including platelets, renal/liver function, and physical signs of bleeding. Gastric protection with PPI or $\mathrm{H} 2$ blockers should be considered in all such patients. 


\section{Optimizing dose adjustments of vitamin-K antagonists}

In spite of the preferred use of NOACs for stroke prevention in eligible patients with $A F,{ }^{3}$ some situations still require the use of VKA, including patients with mechanical heart valves as well as those with $A F$ in the setting of rheumatic mitral stenosis. As such, mastering VKA therapy and dosing to keep patients in the therapeutic range remains an important skillset.

Beyond the standard target INR of 2.0-3.0 much of the optimal management of VKA therapy in AF is experience - rather than evidence-based. As such, various algorithms exist for the management of different VKA ${ }^{464,465}$ and experience in the past decades has led to different clinical routines (e.g. anticoagulation clinics, selfmeasurement via point-of-care devices etc.). One aspect, however, is key to success in VKA treated patients: Maintenance of a high time in therapeutic range (TTR) has been shown to reduce the risk of ischaemic and bleeding events and should be the primary goal in the treatment of these patients independent of the type management approach. Conversely, a change in the approach to these patients needs to be considered if a low TTR is consistently observed.

\section{Dosing during initiation of therapy}

Automated dosing calculators are available that help in the determination of the 'optimal' starting regimen (e.g. http://www.warfarindos ing.org). One randomized trial comparing a $10 \mathrm{mg}$ and 5-mg Warfarin Initiation Nomograms for the outpatient treatment of acute VTE suggested the $10 \mathrm{mg}$ scheme to be superior with patients reaching a therapeutic INR faster. ${ }^{466}$ However, a meta-analysis found no evidence of superiority of either starting regimen. ${ }^{467}$ Moreover, the situation is different in patients with AF as they are generally older and more frail than VTE patients. Furthermore, AF patients are usually not initiated in the setting of an acute thrombotic event. Indeed, various factors may play in favour of using a low (or even lower, i.e. $2 \mathrm{mg}$ qd) starting dose, including older age, frailty, and renal insufficiency. As such, no strong recommendation can be made for routinely using either strategy, and individualizing the approach based on patient characteristics is recommended. In view of the lack of evidence supporting genotype-based dosing the latter is not recommended on a general basis. ${ }^{465,468}$

In many parts of Europe, anticoagulation with phenprocoumon is frequently started with a loading dose in order to shorten the time to therapeutic INR levels owing to the long half-life of the drug, ${ }^{469}$ whereas the situation for warfarin and acenocoumarol is less clear. ${ }^{470}$ In order to prevent a possible transient prothrombotic effect due to a reduction of the equally vitamin $\mathrm{K}$ dependent, anticoagulant protein $\mathrm{C}$ (and S), the first phase of anticoagulation (particularly with phenprocoumon) is frequently paralleled by a parenteral anticoagulant, but evidence for the superiority of routinely using this approach is missing.

\section{Dosing during maintenance therapy}

Interpatient variability of optimal warfarin dose is enormous. Even in (formerly) 'stable' patients, intercurrent illness, change in dietary habits, changes in co-medication etc. may have a substantial impact on INR values. Despite the large variation of warfarin dosing habits amongst different centres, data have emerged indicating the
Table 17 Maintenance Warfarin dosing for out-oftherapeutic-range international normalized ratio

\begin{tabular}{|l|l|}
\hline INR & Dose adjustment per week \\
\hline$\leq 1.5$ & $\uparrow$ by $15 \% /$ week \\
\hline $1.6-1.9$ & $\uparrow$ by $10 \% /$ week \\
\hline $2-2.9$ & Unchanged \\
\hline $3-3.9$ & $\downarrow$ by $10 \% /$ week \\
\hline $4-4.9$ & Hold 1 dose, then restart with dose $\downarrow$ by $10 \% /$ week \\
\hline$\geq 5$ & $\begin{array}{l}\text { Hold until INR is } 2-3 \text {, then restart with } \\
\text { dose } \downarrow \text { by } 15 \% / \text { week }\end{array}$ \\
\hline
\end{tabular}

Suggested dose adjustment in case of out-of-therapeutic-range INR. ${ }^{472}$ Importantly, dosing is optimized not using daily dose adjustments but adjustments based on the weekly intake in warfarin.

usefulness of using dosing algorithms to optimize VKA dosing and, ultimately, the time in therapeutic range (TTR). ${ }^{471-473}$ One such algorithm is presented in Table 17, derived from the warfarin arm of the RE-LY trial. Importantly from a conceptual point of view dosing is optimized not using daily dose adjustments but adjustments based on the weekly intake in warfarin. Obtaining INR measurements at least every 4 weeks and at least weekly in case of out-of-range values is an important prerequisite. A similar dosing scheme may be used for phenprocoumon given its even longer half-life, whereas for acenocoumarol more short-term based adjustment may be feasible given its shorter half-life.

In patients with repeated out-of-range INR values, supplemental measures may be required including (re-)educating patients on the risk and benefits of VKA intake, the importance of strict adherence as well as food-and drug-drug interactions etc. Receiving care at a dedicated anticoagulation clinic ${ }^{474,475}$ as well as self-monitoring and selfmanagement ${ }^{476}$ has been shown to improve INR control. However, patient selection is a critical component, particularly for the latter, and not every patient may be suitable.

In summary, every effort needs to be made in VKA treated patients to optimize the individual patient's TTR. At the same time, however, it needs to be kept in mind that even being within the therapeutic range does not protect from bleeding events. Recent studies indicate that although the risk of ICB increases at an INR $>3$ (and clearly $>4-5$ ), the vast majority of events in absolute numbers occurs at a therapeutic INR level. ${ }^{377}$ Keeping the patient in the therapeutic range (2.0-3.0) hence primarily confers relative, but not absolute efficacy and safety.

\section{Acknowledgements}

The authors thank EHRA Scienfitic Documents committee: Gregory Y.H. Lip, Laurent Fauchier, David Arnar, Carina BlomstromLundqvist, Zbigniew Kalarus, Gulmira Kudaiberdieva, Georges H. Mairesse, Tatjana Potpara, Irina Savelieva, Jesper Hastrup Svendsen, Vassil B. Traykov. 


\section{Funding}

This article and derived educational materials (slide set, website, booklet, and NOAC card) were produced by and under the sole responsibility of the European Heart Rhythm Association, and supported by Bayer Pharma AG, Boehringer Ingelheim, Bristol-Myers Squibb, and Pfizer Alliance and Daiichi-Sankyo Europe $\mathrm{GmbH}$ in the form of an Unrestricted Educational Grant.

The EHRA writing committee collaborated with medical advisors from the different companies to assure data accuracy and completeness.

Conflict of interest: J. S. has received consultant and/or speaker fees from Amgen, Astra-Zeneca, Atricure, Bayer, Biosense Webster, Biotronik, Boehringer-Ingelheim, Boston Scientific, Bristol-Myers Squibb, Cook Medical, Daiichi Sankyo, Medtronic, Novartis, Pfizer, Sanofi-Aventis, Sorin, St. Jude Medical/Abbott, and Zoll. He reports ownership of CorXL. J.S. has received grant support through his institution from Bayer Healthcare, Biosense Webster, Biotronik, Boston Scientific, Daiichi Sankyo, Medtronic, and St. Jude Medical/Abbott. P. V. reports grants and personal fees from Bayer, Boehringer Ingelheim, BMS, Leo Pharma, Daiichi-Sankyo; and personal fees from Pfizer, Medtronic, and Portola. T.S. P. has received speaker fees from Pfizer and Bayer. P. A. reports personal fees from Boehringer Ingelheim, personal fees and non-financial support from Bayer and Portola, and grants, personal fees and non-financial support from Pfizer-BMS and Csl-Behring. M. A. reports personal fees from Bayer, Boehringer Ingelheim, Daiichi Sankyo, personal fees from Pfizer, Sanofi Aventis, Medtronic, Biosense Webster, Novartis, Abbott, and Biotronik. L.D. has no conflict of interest to disclose. K.G. H. reports personal fees from Bayer, Boehringer-Ingelheim, Pfizer, Bristol-Myers Squibb, Daiichi Sankyo, Medtronic, Edwards Lifesciences, Sanofi, EIP Pharma, non-financial support from Getemed AG, and grants from Bayer. J. O. reports fees to his institution from Bayer, Boehringer Ingelheim, Bristol-Myers Squibb, Daichii-Sankyo, Pfizer, and Sanofi. H. R. reports personal fees from BMS, MedUpdate, NephroUpdate, Pfizer, and Pluristem; and grants from the German Federal Ministry for Education and Research (BMBF), Bard, Bayer, and Biotronik. V. R. has received consultant and/or speaker fees from Bayer Healthcare, Boehringer-Ingelheim, Bristol-Myers Squibb, Leo Pharma, Pfizer, and Rovi. N. R. reports personal fees from BMS, Pfizer, Daiichi-Sankyo, Bayer and Boehringer Ingelheim. P. S. reports grants from Daiichi Sankyo and Astra Zeneca, and institutional fees from BMS, Pfizer, and Boehringer. R. C. reports personal fees from Daichii Sankyo, Pfizer, Bayer and Boehringer Ingelheim. A. J.C. has received institutional research grants and personal fees for advice and for speaking from Bayer, Boehringer Ingelheim, Daiichi Sankyo, and Pfizer/BMS. H. H. reports personal fees (before June 2017) from Abbott, Pfizer/BMS, Daiichi-Sankyo, Boehringer-Ingelheim, Cardiome; he received no personal fees after June 2017; he received research grants from Bayer, Bracco Imaging Europe, Medtronic, and St. Jude Medical through the University of Hasselt or Antwerp.

\section{References}

1. Heidbuchel H, Verhamme P, Alings M, Antz M, Hacke W, Oldgren J, Sinnaeve P, Camm AJ, Kirchhof P; European Heart Rhythm Association. European Heart Rhythm Association Practical Guide on the use of new oral anticoagulants in patients with non-valvular atrial fibrillation. Europace 2013;15:625-651.

2. Heidbuchel H, Verhamme P, Alings M, Antz M, Diener HC, Hacke W, Oldgren J, Sinnaeve P, Camm AJ, Kirchhof P. Updated European Heart Rhythm Association Practical Guide on the use of non-vitamin K antagonist anticoagulants in patients with non-valvular atrial fibrillation. Europace 2015;17:1467-1507.

3. Kirchhof P, Benussi S, Kotecha D, Ahlsson A, Atar D, Casadei B, Castella M, Diener HC, Heidbuchel H, Hendriks J, Hindricks G, Manolis AS, Oldgren J, Popescu BA, Schotten U, Van Putte B, Vardas P, Agewall S, Camm J, Baron Esquivias G, Budts W, Carerj S, Casselman F, Coca A, De Caterina R,
Deftereos S, Dobrev D, Ferro JM, Filippatos G, Fitzsimons D, Gorenek B, Guenoun M, Hohnloser SH, Kolh P, Lip GY, Manolis A, McMurray J, Ponikowski P, Rosenhek R, Ruschitzka F, Savelieva I, Sharma S, Suwalski P, Tamargo JL, Taylor CJ, Van Gelder IC, Voors AA, Windecker S, Zamorano JL, Zeppenfeld K. 2016 ESC Guidelines for the management of atrial fibrillation developed in collaboration with EACTS. Eur Heart J 2016;37:2893-2962.

4. Barnes GD, Ageno W, Ansell J, Kaatz S; Subcommittee on the Control of Anticoagulation of the International Society on Thrombosis and Haemostasis. Recommendation on the Nomenclature for Oral Anticoagulants: communication from the SSC of the ISTH. J Thromb Haemost 2015;13:1154-1156.

5. Baumgartner H, Falk V, Bax JJ, De Bonis M, Hamm C, Holm PJ, lung B, Lancellotti P, Lansac E, Munoz DR, Rosenhek R, Sjogren J, Tornos Mas P, Vahanian A, Walther T, Wendler O, Windecker S, Zamorano JL; ESC Scientific Document Group. 2017 ESC/EACTS Guidelines for the management of valvular heart disease. Eur Heart / 2017;38:2739-2791.

6. Lip GYH, Collet JP, Caterina R, Fauchier L, Lane DA, Larsen TB, Marin F, Morais J, Narasimhan C, Olshansky B, Pierard L, Potpara T, Sarrafzadegan N, Sliwa K, Varela G, Vilahur G, Weiss T, Boriani G, Rocca B; ESC Scientific Document Group. Antithrombotic therapy in atrial fibrillation associated with valvular heart disease: a joint consensus document from the European Heart Rhythm Association (EHRA) and European Society of Cardiology Working Group on Thrombosis, endorsed by the ESC Working Group on Valvular Heart Disease, Cardiac Arrhythmia Society of Southern Africa (CASSA), Heart Rhythm Society (HRS), Asia Pacific Heart Rhythm Society (APHRS), South African Heart (SA Heart) Association and Sociedad Latinoamericana de Estimulacion Cardiaca y Electrofisiologia (SOLEACE). Europace 2017;19:1757-1758.

7. Avezum A, Lopes RD, Schulte PJ, Lanas F, Gersh BJ, Hanna M, Pais P, Erol C, Diaz R, Bahit MC, Bartunek J, De Caterina R, Goto S, Ruzyllo W, Zhu J, Granger $\mathrm{CB}$, Alexander JH. Apixaban in comparison with warfarin in patients with atrial fibrillation and valvular heart disease: findings from the Apixaban for Reduction in Stroke and Other Thromboembolic Events in Atrial Fibrillation (ARISTOTLE) Trial. Circulation 2015;132:624-632.

8. Ezekowitz MD, Nagarakanti R, Noack H, Brueckmann M, Litherland C, Jacobs M, Clemens A, Reilly PA, Connolly SJ, Yusuf S, Wallentin L. Comparison of dabigatran and warfarin in patients with atrial fibrillation and valvular heart disease: the RE-LY Trial (Randomized Evaluation of Long-Term Anticoagulant Therapy). Circulation 2016;134:589-598.

9. Breithardt G, Baumgartner H, Berkowitz SD, Hellkamp AS, Piccini JP, Stevens SR, Lokhnygina Y, Patel MR, Halperin JL, Singer DE, Hankey GJ, Hacke W, Becker RC, Nessel CC, Mahaffey KW, Fox KA, Califf RM; ROCKET AF Steering Committee \& Investigators. Clinical characteristics and outcomes with rivaroxaban vs. warfarin in patients with non-valvular atrial fibrillation but underlying native mitral and aortic valve disease participating in the ROCKET AF trial. Eur Heart J 2014;35:3377-3385.

10. De Caterina R, Renda G, Carnicelli AP, Nordio F, Trevisan M, Mercuri MF, Ruff CT, Antman EM, Braunwald E, Giugliano RP. Valvular heart disease patients on edoxaban or warfarin in the ENGAGE AF-TIMI 48 Trial. J Am Coll Cardiol 2017;69:1372-1382

11. Pan KL, Singer DE, Ovbiagele B, Wu YL, Ahmed MA, Lee M. Effects of nonvitamin $\mathrm{K}$ antagonist oral anticoagulants versus warfarin in patients with atrial fibrillation and valvular heart disease: a systematic review and meta-analysis. J Am Heart Assoc 2017; 6:e005835.

12. Renda G, Ricci F, Giugliano RP, De Caterina R. Non-vitamin K antagonist oral anticoagulants in patients with atrial fibrillation and valvular heart disease. J Am Coll Cardiol 2017;69:1363-1371.

13. Noseworthy PA, Yao X, Shah ND, Gersh BJ. Comparative effectiveness and safety of non-vitamin $\mathrm{K}$ antagonist oral anticoagulants versus warfarin in patients with atrial fibrillation and valvular heart disease. Int I Cardiol 2016;209:181-183.

14. Noseworthy PA, Yao X, Shah ND, Gersh BJ. Stroke and bleeding risks in NOAC- and warfarin-treated patients with hypertrophic cardiomyopathy and atrial fibrillation. J Am Coll Cardiol 2016;67:3020-3021.

15. Dominguez F, Climent V, Zorio E, Ripoll-Vera T, Salazar-Mendiguchía J, García-Pinilla JM, Urbano-Moral JA, Fernández-Fernández X, Lopez-Cuenca D, Ajo-Ferrer R, Sanz-Sanchez J, Gomez-Perez Y, López-Garrido MA, BarrialesVilla R, Gimeno JR, Garcia-Pavia P. Direct oral anticoagulants in patients with hypertrophic cardiomyopathy and atrial fibrillation. Int I Cardiol 2017; 248:232-238.

16. van Diepen S, Hellkamp AS, Patel MR, Becker RC, Breithardt G, Hacke W, Halperin JL, Hankey GJ, Nessel CC, Singer DE, Berkowitz SD, Califf RM, Fox $K A$, Mahaffey KW. Efficacy and safety of rivaroxaban in patients with heart failure and nonvalvular atrial fibrillation: insights from ROCKET AF. Circ Heart Fail 2013;6:740-747.

17. McMurray JJ, Ezekowitz JA, Lewis BS, Gersh BJ, van Diepen S, Amerena J, Bartunek J, Commerford P, Oh BH, Harjola VP, Al-Khatib SM, Hanna M, 
Alexander JH, Lopes RD, Wojdyla DM, Wallentin L, Granger CB; ARISTOTLECommittees and Investigators. Left ventricular systolic dysfunction, heart failure, and the risk of stroke and systemic embolism in patients with atrial fibrillation: insights from the ARISTOTLE trial. Circ Heart Fail 2013;6: $451-460$.

18. Magnani G, Giugliano RP, Ruff CT, Murphy SA, Nordio F, Metra M, Moccetti T, Mitrovic V, Shi M, Mercuri M, Antman EM, Braunwald E. Efficacy and safety of edoxaban compared with warfarin in patients with atrial fibrillation and heart failure: insights from ENGAGE AF-TIMI 48. Eur J Heart Fail 2016;18:1153-1161.

19. Lane DA, Aguinaga L, Blomstrom-Lundqvist C, Boriani G, Dan GA, Hills MT, Hylek EM, LaHaye SA, Lip GY, Lobban T, Mandrola J, McCabe PJ, Pedersen SS, Pisters R, Stewart S, Wood K, Potpara TS, Document R, Gorenek B, Conti JB, Keegan R, Power S, Hendriks J, Ritter P, Calkins H, Violi F, Hurwitz J. Cardiac tachyarrhythmias and patient values and preferences for their management: the European Heart Rhythm Association (EHRA) consensus document endorsed by the Heart Rhythm Society (HRS), Asia Pacific Heart Rhythm Society (APHRS), and Sociedad Latinoamericana de Estimulacion Cardiaca y Electrofisiologia (SOLEACE). Europace 2015;17:1747-1769.

20. Heidbuchel H, Berti D, Campos M, Desteghe L, Freixo A, Nunes A, Roldan V, Toschi V, Lassila R. Implementation of non-vitamin $\mathrm{K}$ antagonist oral anticoagulants in daily practice: the need for comprehensive education for professionals and patients. Thromb J 2015;13:22.

21. Apostolakis S, Sullivan RM, Olshansky B, Lip GY. Factors affecting quality of anticoagulation control among patients with atrial fibrillation on warfarin: the SAMe-TT(2)R(2) score. Chest 2013;144:1555-1563.

22. Ruiz-Ortiz M, Bertomeu V, Cequier Á, Marín F, Anguita M. Validation of the SAMe-TT2R2 score in a nationwide population of nonvalvular atrial fibrillation patients on vitamin $\mathrm{K}$ antagonists. Thromb Haemost 2015;114:695-701.

23. Roldan V, Cancio S, Galvez J, Valdes M, Vicente V, Marin F, Lip GY. The SAMeTTR score predicts poor anticoagulation control in AF patients: a prospective 'Real-World' inception cohort study. Am J Med 2015;128:1237-1243.

24. Esteve-Pastor MA, Roldán V, Valdés M, Lip GYH, Marín F. The SAMe-TT2R2 score and decision-making between a vitamin $\mathrm{K}$ antagonist or a non-vitamin $\mathrm{K}$ antagonist oral anticoagulant in patients with atrial fibrillation. Expert Rev Cardiovasc Ther 2016;14:177-187.

25. Lobos-Bejarano JM, Barrios V, Polo-García J, Escobar C, Vargas-Ortega D, Marín-Montañés N, Prieto-Valiente L, Fuentes S, Prieto MA, García-Ortiz L. Evaluation of SAMe-TT2R2 score and other clinical factors influencing the quality of anticoagulation therapy in non-valvular atrial fibrillation: a nationwide study in Spain. Curr Med Res Opin 2016;32:1201-1207.

26. Abumuaileq RR, Abu-Assi E, Raposeiras-Roubin S, Lopez-Lopez A, RedondoDieguez A, Alvarez-Iglesias D, Rodriguez-Manero M, Pena-Gil C, GonzalezJuanatey JR. Evaluation of SAMe-TT2R2 risk score for predicting the quality of anticoagulation control in a real-world cohort of patients with non-valvular atrial fibrillation on vitamin-K antagonists. Europace 2015;17:711-717.

27. Proietti M, Lip GY. Simple decision-making between a vitamin $K$ antagonist and a non-vitamin $\mathrm{K}$ antagonist oral anticoagulant: using the SAMe-TT2R2 score. Eur Heart I Cardiovasc Pharmacother 2015;1:150-152.

28. Connolly SJ, Ezekowitz MD, Yusuf S, Eikelboom J, Oldgren J, Parekh A, Pogue J, Reilly PA, Themeles E, Varrone J, Wang S, Alings M, Xavier D, Zhu J, Diaz R, Lewis BS, Darius H, Diener HC, Joyner CD, Wallentin L. Dabigatran versus warfarin in patients with atrial fibrillation. N Engl J Med 2009;361:1139-1151.

29. Patel MR, Mahaffey KW, Garg J, Pan G, Singer DE, Hacke W, Breithardt G, Halperin JL, Hankey GJ, Piccini JP, Becker RC, Nessel CC, Paolini JF, Berkowitz SD, Fox KA, Califf RM. Rivaroxaban versus warfarin in nonvalvular atrial fibrillation. N Engl J Med 2011;365:883-891.

30. Granger CB, Alexander JH, McMurray JJ, Lopes RD, Hylek EM, Hanna M, AlKhalidi HR, Ansell J, Atar D, Avezum A, Bahit MC, Diaz R, Easton JD, Ezekowitz JA, Flaker G, Garcia D, Geraldes M, Gersh BJ, Golitsyn S, Goto S, Hermosillo AG, Hohnloser SH, Horowitz J, Mohan P, Jansky P, Lewis BS, Lopez-Sendon JL, Pais P, Parkhomenko A, Verheugt FW, Zhu J, Wallentin L. Apixaban versus warfarin in patients with atrial fibrillation. $N$ Engl J Med 2011;365:981-992.

31. Giugliano RP, Ruff CT, Braunwald E, Murphy SA, Wiviott SD, Halperin JL, Waldo AL, Ezekowitz MD, Weitz JI, Spinar J, Ruzyllo W, Ruda M, Koretsune Y, Betcher J, Shi M, Grip LT, Patel SP, Patel I, Hanyok JJ, Mercuri M, Antman EM. Edoxaban versus warfarin in patients with atrial fibrillation. $N$ Engl J Med 2013;369: 2093-2104

32. Valgimigli M, Bueno H, Byrne RA, Collet JP, Costa F, Jeppsson A, Juni P, Kastrati A, Kolh P, Mauri L, Montalescot G, Neumann FJ, Petricevic M, Roffi M, Steg PG, Windecker S, Zamorano JL. 2017 ESC focused update on dual antiplatelet therapy in coronary artery disease developed in collaboration with EACTS: the Task Force for dual antiplatelet therapy in coronary artery disease of the European Society of Cardiology (ESC) and of the European Association for Cardio-Thoracic Surgery (EACTS). Eur Heart J 2018;39:123-260.
33. Ibanez B, James S, Agewall S, Antunes MJ, Bucciarelli-Ducci C, Bueno $H$, Caforio ALP, Crea F, Goudevenos JA, Halvorsen S, Hindricks G, Kastrati A, Lenzen MJ, Prescott E, Roffi M, Valgimigli M, Varenhorst C, Vranckx P, Widimsky P. 2017 ESC Guidelines for the management of acute myocardial infarction in patients presenting with ST-segment elevation: the Task Force for the management of acute myocardial infarction in patients presenting with STsegment elevation of the European Society of Cardiology (ESC). Eur Heart J 2018;39:119-177.

34. Scarpignato C, Gatta L, Zullo A, Blandizzi C. Effective and safe proton pump inhibitor therapy in acid-related diseases-a position paper addressing benefits and potential harms of acid suppression. BMC Med 2016;14:179.

35. Ray WA, Chung CP, Murray KT, Smalley WE, Daugherty JR, Dupont WD, Stein $\mathrm{CM}$. Association of proton pump inhibitors with reduced risk of warfarinrelated serious upper gastrointestinal bleeding. Gastroenterology 2016;151: 1105-1112.e10.

36. Di Minno A, Spadarella G, Spadarella E, Tremoli E, Di Minno G. Gastrointestinal bleeding in patients receiving oral anticoagulation: current treatment and pharmacological perspectives. Thromb Res 2015;136:1074-1081.

37. Chan EW, Lau WC, Leung WK, Mok MT, He Y, Tong TS, Wong IC. Prevention of dabigatran-related gastrointestinal bleeding with gastroprotective agents: a population-based study. Gastroenterology 2015;149:586-595.e3.

38. Shields AM, Lip GY. Choosing the right drug to fit the patient when selecting oral anticoagulation for stroke prevention in atrial fibrillation. J Intern Med 2015;278:1-18.

39. Okumura K, Hori M, Tanahashi N, John Camm A. Special considerations for therapeutic choice of non-vitamin $\mathrm{K}$ antagonist oral anticoagulants for Japanese patients with nonvalvular atrial fibrillation. Clin Cardiol 2017;40:126-131.

40. Diener HC, Aisenberg J, Ansell J, Atar D, Breithardt G, Eikelboom J, Ezekowitz MD, Granger CB, Halperin JL, Hohnloser SH, Hylek EM, Kirchhof P, Lane DA, Verheugt FWA, Veltkamp R, Lip GYH. Choosing a particular oral anticoagulant and dose for stroke prevention in individual patients with non-valvular atrial fibrillation: part 1. Eur Heart J 2017;38:852-859.

41. Diener HC, Aisenberg J, Ansell J, Atar D, Breithardt G, Eikelboom J, Ezekowitz MD, Granger CB, Halperin JL, Hohnloser SH, Hylek EM, Kirchhof P, Lane DA Verheugt FWA, Veltkamp R, Lip GYH. Choosing a particular oral anticoagulant and dose for stroke prevention in individual patients with non-valvular atrial fibrillation: part 2. Eur Heart J 2017;38:860-868.

42. Lane DA, Barker RV, Lip GY. Best practice for atrial fibrillation patient education. Curr Pharm Des 2015;21:533-543.

43. Lane DA, Wood K. Cardiology patient page. Patient guide for taking the nonvitamin $\mathrm{K}$ antagonist oral anticoagulants for atrial fibrillation. Circulation 2015;131:e412-e415.

44. Camm AJ, Accetta G, Ambrosio G, Atar D, Bassand J-P, Berge E, Cools F, Fitzmaurice DA, Goldhaber SZ, Goto S, Haas S, Kayani G, Koretsune Y, Mantovani LG, Misselwitz F, Oh S, Turpie AGG, Verheugt FWA, Kakkar AK. Evolving antithrombotic treatment patterns for patients with newly diagnosed atrial fibrillation. Heart 2017;103:307-314.

45. Huisman MV, Rothman KJ, Paquette M, Teutsch C, Diener H-C, Dubner SJ, Halperin JL, Ma CS, Zint K, Elsaesser A, Bartels DB, Lip GYH. The changing landscape for stroke prevention in AF: findings from the GLORIA-AF Registry Phase 2. J Am Coll Cardiol 2017;69:777-785.

46. Berti D, Hendriks JM, Brandes A, Deaton C, Crijns HJ, Camm AJ, Hindricks G, Moons P, Heidbuchel H. A proposal for interdisciplinary, nurse-coordinated atrial fibrillation expert programmes as a way to structure daily practice. Eur Heart J 2013;34:2725-2730.

47. Hendriks JM, de Wit R, Crijns HJ, Vrijhoef HJ, Prins MH, Pisters R, Pison LA, Blaauw $Y$, Tieleman RG. Nurse-led care vs. usual care for patients with atrial fibrillation: results of a randomized trial of integrated chronic care vs. routine clinical care in ambulatory patients with atrial fibrillation. Eur Heart J 2012;33:2692-2699.

48. Gallagher C, Elliott AD, Wong CX, Rangnekar G, Middeldorp ME, Mahajan R, Lau DH, Sanders P, Hendriks JML. Integrated care in atrial fibrillation: a systematic review and meta-analysis. Heart 2017;103:1947-1953.

49. Carter L, Gardner M, Magee K, Fearon A, Morgulis I, Doucette S, Sapp JL, Gray C, Abdelwahab A, Parkash R. An integrated management approach to atrial fibrillation. J Am Heart Assoc 2016;5:e002950.

50. Kirchhof $P$. The future of atrial fibrillation management: integrated care and stratified therapy. Lancet 2017;390:1873-1887.

51. Desteghe L, Vijgen J, Koopman P, Dilling-Boer D, Schurmans J, Dendale P, Heidbuchel $H$. Telemonitoring-based feedback improves adherence to nonvitamin $\mathrm{K}$ antagonist oral anticoagulants intake in patients with atrial fibrillation. Eur Heart J 2018;39:1394-1403.

52. Steffel J, Giugliano RP, Braunwald E, Murphy SA, Mercuri M, Choi Y, Aylward P, White H, Zamorano JL, Antman EM, Ruff CT. Edoxaban versus warfarin in atria fibrillation patients at risk of falling: ENGAGE AF-TIMI 48 analysis. J Am Coll Cardiol 2016;68:1169-1178. 
53. Romero-Ortuno R, Walsh CD, Lawlor BA, Kenny RA. A frailty instrument for primary care: findings from the Survey of Health, Ageing and Retirement in Europe (SHARE). BMC Geriatr 2010;10:57.

54. Chao TF, Lip GYH, Liu C], Lin YJ, Chang SL, Lo LW, Hu YF, Tuan TC, Liao JN, Chung FP, Chen TJ, Chen SA. Relationship of aging and incident comorbidities to stroke risk in patients with atrial fibrillation. I Am Coll Cardiol 2018;71:122-132.

55. Lip GYH, Skjoth F, Nielsen PB, Kjaeldgaard JN, Larsen TB. The HAS-BLED, ATRIA, and ORBIT bleeding scores in atrial fibrillation patients using non-vitamin K antagonist oral anticoagulants. Am J Med 2017; [Epub ahead of print].

56. Guo Y, Zhu H, Chen Y, Lip GYH. Comparing bleeding risk assessment focused on modifiable risk factors only versus validated bleeding risk scores in atrial fibrillation. Am J Med 2018;131:185-192.

57. Esteve-Pastor M, Rivera-Caravaca J, Shantsila A, Roldán V, Lip G, Marín F. Assessing bleeding risk in atrial fibrillation patients: comparing a bleeding risk score based only on modifiable bleeding risk factors against the HAS-BLED Score. The AMADEUS Trial. Thromb Haemost 2017;117:22612266.

58. Chao TF, Lip GYH, Lin YJ, Chang SL, Lo LW, Hu YF, Tuan TC, Liao JN, Chung FP, Chen TJ, Chen SA. Major bleeding and intracranial hemorrhage risk prediction in patients with atrial fibrillation: attention to modifiable bleeding risk factors or use of a bleeding risk stratification score? A nationwide cohort study. Int J Cardiol 2018:254:157-161.

59. Hijazi Z, Oldgren J, Lindbäck J, Alexander JH, Connolly SJ, Eikelboom JW, Ezekowitz MD, Held C, Hylek EM, Lopes RD, Siegbahn A, Yusuf S, Granger CB, Wallentin L. ARISTOTLE and RE-LY Investigators. Lancet 2016;387:2302-2311.

60. O'Brien EC, Simon DN, Thomas LE, Hylek EM, Gersh BJ, Ansell JE, Kowey PR, Mahaffey KW, Chang P, Fonarow GC, Pencina MJ, Piccini JP, Peterson ED. The ORBIT bleeding score: a simple bedside score to assess bleeding risk in atrial fibrillation. Eur Heart / 2015;36:3258-3264.

61. Lip GY, Lane DA. Bleeding risk assessment in atrial fibrillation: observations on the use and misuse of bleeding risk scores. I Thromb Haemost 2016;14:1711-1714.

62. Raparelli V, Proietti M, Cangemi R, Lip GY, Lane DA, Basili S. Adherence to oral anticoagulant therapy in patients with atrial fibrillation. Focus on nonvitamin $\mathrm{K}$ antagonist oral anticoagulants. Thromb Haemost 2016;117:209-218.

63. Montalescot G. Abstract 18842: adherence and persistence to apixaban treatment in patients with non valvular atrial fibrillation is high and similar with standard of care patient education or with an additional educational program: the randomized AEGEAN Study. Circulation 2016;134(Suppl 1): A18842A18842.

64. Desteghe L, Engelhard L, Vijgen J, Koopman P, Dilling-Boer D, Schurmans J, Dendale $\mathrm{P}$, Heidbuchel $\mathrm{H}$. Effect of individualised education sessions on the knowledge level of patients with atrial fibrillation. EP Europace 2017;19:iii147. P817.

65. Labovitz DL, Shafner L, Reyes Gil M, Virmani D, Hanina A. Using artificial intelligence to reduce the risk of nonadherence in patients on anticoagulation therapy. Stroke 2017;48:1416-1419.

66. Yao X, Abraham NS, Alexander GC, Crown W, Montori VM, Sangaralingham LR, Gersh BJ, Shah ND, Noseworthy PA. Effect of adherence to oral anticoagulants on risk of stroke and major bleeding among patients with atrial fibrillation. | Am Heart Assoc 2016:5:e003074.

67. Beyer-Westendorf J, Ehlken B, Evers T. Real-world persistence and adherence to oral anticoagulation for stroke risk reduction in patients with atrial fibrillation. Europace 2016;18:1150-1157.

68. Shore S, Carey EP, Turakhia MP, Jackevicius CA, Cunningham F, Pilote L, Bradley SM, Maddox TM, Grunwald GK, Baron AE, Rumsfeld JS, Varosy PD, Schneider PM, Marzec LN, Ho PM. Adherence to dabigatran therapy and longitudinal patient outcomes: insights from the veterans health administration. Am Heart J 2014;167:810-817.

69. Gorst-Rasmussen A, Skjoth F, Larsen TB, Rasmussen LH, Lip GY, Lane DA. Dabigatran adherence in atrial fibrillation patients during the first year after diagnosis: a nationwide cohort study. J Thromb Haemost 2015;13:495-504.

70. McHorney CA, Crivera C, Laliberte F, Nelson WW, Germain G, Bookhart B, Martin S, Schein J, Lefebvre P, Deitelzweig S. Adherence to non-vitamin-Kantagonist oral anticoagulant medications based on the Pharmacy Quality Alliance measure. Curr Med Res Opin 2015;31:2167-2173.

71. Crivera C, Nelson WW, Bookhart B, Martin S, Germain G, Laliberte F, Schein J, Lefebvre P. Pharmacy quality alliance measure: adherence to non-warfarin oral anticoagulant medications. Curr Med Res Opin 2015;31:1889-1895.

72. Coleman Cl, Tangirala M, Evers T. Medication adherence to rivaroxaban and dabigatran for stroke prevention in patients with non-valvular atrial fibrillation in the United States. Int J Cardiol 2016;212:171-173.

73. Alberts MJ, Peacock WF, Fields LE, Bunz TJ, Nguyen E, Milentijevic D, Schein JR, Coleman $\mathrm{Cl}$. Association between once- and twice-daily direct oral anticoagulant adherence in nonvalvular atrial fibrillation patients and rates of ischemic stroke. Int / Cardiol 2016;215(Suppl C):11-13.

74. McHorney CA, Peterson ED, Laliberte F, Germain G, Nelson WW, Crivera C, Schein J, Lefebvre P. Comparison of adherence to rivaroxaban versus apixaban among patients with atrial fibrillation. Clin Ther 2016;38:2477-2488.

75. Brown JD, Shewale AR, Talbert JC. Adherence to rivaroxaban, dabigatran, and apixaban for stroke prevention in incident, treatment-naive nonvalvular atrial fibrillation. J Manag Care Spec Pharm 2016;22:1319-1329.

76. Zhou M, Chang HY, Segal JB, Alexander GC, Singh S. Adherence to a novel oral anticoagulant among patients with atrial fibrillation. I Manag Care Spec Pharm 2015;21:1054-1062

77. Manzoor BS, Lee TA, Sharp LK, Walton SM, Galanter WL, Nutescu EA. Realworld adherence and persistence with direct oral anticoagulants in adults with atrial fibrillation. Pharmacotherapy 2017;37:1221-1230.

78. Cutler TW, Chuang A, Huynh TD, Witt RG, Branch J, Pon T, White R. A retrospective descriptive analysis of patient adherence to dabigatran at a large academic medical center. J Manag Care Spec Pharm 2014;20:1028-1034.

79. Graham DJ, Reichman ME, Wernecke M, Zhang R, Southworth MR, Levenson M, Sheu TC, Mott K, Goulding MR, Houstoun M, MaCurdy TE, Worrall C, Kelman JA. Cardiovascular, bleeding, and mortality risks in elderly Medicare patients treated with dabigatran or warfarin for nonvalvular atrial fibrillation. Circulation 2015:131:157-164.

80. Larsen TB, Rasmussen LH, Skjoth F, Due KM, Callreus T, Rosenzweig M, Lip GY. Efficacy and safety of dabigatran etexilate and warfarin in 'real-world' patients with atrial fibrillation: a prospective nationwide cohort study. J Am Coll Cardiol 2013;61:2264-2273.

81. Lip GY, Keshishian A, Kamble S, Pan X, Mardekian J, Horblyuk R, Hamilton M. Real-world comparison of major bleeding risk among non-valvular atrial fibrillation patients initiated on apixaban, dabigatran, rivaroxaban, or warfarin. A propensity score matched analysis. Thromb Haemost 2016;116:975-986.

82. Adeboyeje G, Sylwestrzak G, Barron J], White J, Rosenberg A, Abarca J, Crawford G, Redberg R. Major bleeding risk during anticoagulation with warfarin, dabigatran, apixaban, or rivaroxaban in patients with nonvalvular atrial fibrillation. J Manag Care Spec Pharm 2017;23:968-978.

83. Bai Y, Deng H, Shantsila A, Lip GYH. Rivaroxaban versus dabigatran or warfarin in real-world studies of stroke prevention in atrial fibrillation: systematic review and meta-analysis. Stroke 2017;48:970-976.

84. Hernandez I, Zhang Y, Saba S. Comparison of the effectiveness and safety of apixaban, dabigatran, rivaroxaban, and warfarin in newly diagnosed atrial fibrillation. Am J Cardiol 2017;120:1813-1819.

85. Bai Y, Shi X-B, Ma C-S, Lip GYH. Meta-analysis of effectiveness and safety of oral anticoagulants in atrial fibrillation with focus on apixaban. Am J Cardiol 2017;120:1689-1695.

86. Staerk L, Fosbøl EL, Lip GYH, Lamberts M, Bonde AN, Torp-Pedersen C, Ozenne B, Gerds TA, Gislason GH, Olesen JB. Ischaemic and haemorrhagic stroke associated with non-vitamin $\mathrm{K}$ antagonist oral anticoagulants and warfarin use in patients with atrial fibrillation: a nationwide cohort study. Europ Heart J 2017;38:907-915.

87. Beyer-Westendorf J, Camm AJ, Coleman Cl, Tamayo S. Rivaroxaban real-world evidence: validating safety and effectiveness in clinical practice. Thromb Haemost 2016;116:S13-S23.

88. Potpara TS, Lip GH. Postapproval observational studies of non-vitamin k antagonist oral anticoagulants in atrial fibrillation. JAMA 2017;317:1115-1116.

89. Friberg L, Oldgren J. Efficacy and safety of non-vitamin $\mathrm{K}$ antagonist oral anticoagulants compared with warfarin in patients with atrial fibrillation. Open Heart 2017;4:e000682.

90. Ntaios G, Papavasileiou V, Makaritsis K, Vemmos K, Michel P, Lip GYH. Realworld setting comparison of nonvitamin-K antagonist oral anticoagulants versus vitamin-K antagonists for stroke prevention in atrial fibrillation: a systematic review and meta-analysis. Stroke 2017:48:2494-2503.

91. Larsen TB, Skjøth F, Nielsen PB, Kjældgaard JN, Lip GY. Comparative effectiveness and safety of non-vitamin $\mathrm{K}$ antagonist oral anticoagulants and warfarin in patients with atrial fibrillation: propensity weighted nationwide cohort study. BMJ 2016;353:13189.

92. Halvorsen S, Ghanima W, Fride Tvete I, Hoxmark C, Falck P, Solli O, Jonasson C. A nationwide registry study to compare bleeding rates in patients with atria fibrillation being prescribed oral anticoagulants. Eur Heart J Cardiovasc Pharmacother 2017;3:28-36.

93. Nielsen PB, Skjoth F, Sogaard M, Kjaeldgaard JN, Lip GY, Larsen TB. Effectiveness and safety of reduced dose non-vitamin $\mathrm{K}$ antagonist oral anticoagulants and warfarin in patients with atrial fibrillation: propensity weighted nationwide cohort study. BMJ 2017;356, https://www.ncbi.nlm.nih.gov/pubmed/ 28188243.

94. Hohnloser SH, Basic E, Nabauer M. Comparative risk of major bleeding with new oral anticoagulants (NOACs) and phenprocoumon in patients with atrial fibrillation: a post-marketing surveillance study. Clin Res Cardiol 2017;106:618-628. 
95. Lamberts M, Staerk L, Olesen JB, Fosbol EL, Hansen ML, Harboe L, Lefevre C, Evans D, Gislason GH. Major bleeding complications and persistence with oral anticoagulation in non-valvular atrial fibrillation: contemporary findings in reallife Danish patients. J Am Heart Assoc 2017;6:e004517.

96. Coleman $\mathrm{Cl}$, Antz M. Real-world evidence with apixaban for stroke prevention in patients with nonvalvular atrial fibrillation in Germany: a retrospective study (REASSESS). Intern Emerg Med 2017;12:419-422.

97. Li XS, Deitelzweig S, Keshishian A, Hamilton M, Horblyuk R, Gupta K, Luo X, Mardekian J, Friend K, Nadkarni A, Pan X, Lip GYH. Effectiveness and safety of apixaban versus warfarin in non-valvular atrial fibrillation patients in 'real-world' clinical practice. A propensity-matched analysis of 76,940 patients. Thromb Haemost 2017;117:1072-1082.

98. Deitelzweig S, Farmer C, Luo X, Li X, Vo L, Mardekian J, Fahrbach K, Ashaye A. Comparison of major bleeding risk in patients with non-valvular atrial fibrillation receiving direct oral anticoagulants in the real-world setting: a network metaanalysis. Curr Med Res Opin 2018;34:487-498.

99. Obamiro KO, Chalmers L, Bereznicki LR. A summary of the literature evaluating adherence and persistence with oral anticoagulants in atrial fibrillation. Am J Cardiovasc Drugs 2016;16:349-363.

100. Martinez C, Katholing A, Wallenhorst C, Freedman SB. Therapy persistence in newly diagnosed non-valvular atrial fibrillation treated with warfarin or NOAC. A cohort study. Thromb Haemost 2016;115:31-39.

101. Nelson WW, Song X, Coleman Cl, Thomson E, Smith DM, Damaraju CV, Schein JR. Medication persistence and discontinuation of rivaroxaban versus warfarin among patients with non-valvular atrial fibrillation. Curr Med Res Opin 2014:30:2461-2469.

102. Laliberte F, Cloutier M, Nelson WW, Coleman Cl, Pilon D, Olson WH, Damaraju CV, Schein JR, Lefebvre P. Real-world comparative effectiveness and safety of rivaroxaban and warfarin in nonvalvular atrial fibrillation patients. Curr Med Res Opin 2014;30:1317-1325.

103. Zalesak M, Siu K, Francis K, Yu C, Alvrtsyan H, Rao Y, Walker D, Sander S, Miyasato G, Matchar D, Sanchez H. Higher persistence in newly diagnosed nonvalvular atrial fibrillation patients treated with dabigatran versus warfarin. Circ Cardiovasc Qual Outcomes 2013;6:567-574.

104. Beyer-Westendorf J, Forster K, Ebertz F, Gelbricht V, Schreier T, Gobelt M, Michalski F, Endig H, Sahin K, Tittl L, Weiss N. Drug persistence with rivaroxaban therapy in atrial fibrillation patients-results from the Dresden noninterventional oral anticoagulation registry. Europace 2015;17:530-538.

105. Tsai K, Erickson SC, Yang J, Harada AS, Solow BK, Lew HC. Adherence, persistence, and switching patterns of dabigatran etexilate. Am J Manag Care 2013;19:e325-e332.

106. Jackevicius CA, Tsadok MA, Essebag V, Atzema C, Eisenberg MJ, Tu JV, Lu L, Rahme E, Ho PM, Turakhia M, Humphries KH, Behlouli H, Zhou L, Pilote L. Early non-persistence with dabigatran and rivaroxaban in patients with atrial fibrillation. Heart 2017;103:1331-1338.

107. Paquette M, Riou Franca L, Teutsch C, Diener HC, Lu S, Dubner SJ, Ma CS, Rothman KJ, Zint K, Halperin JL, Huisman MV, Lip GYH, Nieuwlaat R. Persistence with dabigatran therapy at 2 years in patients with atrial fibrillation. J Am Coll Cardiol 2017;70:1573-1583.

108. Lane DA, Wood KA. A patient's guide to taking the non-vitamin $\mathrm{K}$ antagonist oral anticoagulants (NOACs) for atrial fibrillation: lane; patient's guide to taking NOACs. Circulation 2015;131:e412-e415.

109. Desteghe L, Engelhard L, Raymaekers Z, Kluts K, Vijgen J, Dilling-Boer D, Koopman P, Schurmans J, Dendale P, Heidbuchel H. Knowledge gaps in patients with atrial fibrillation revealed by a new validated knowledge questionnaire. Int J Cardiol 2016;223:906-914.

110. Vinereanu D, Lopes RD, Bahit MC, Xavier D, Jiang J, Al-Khalidi HR, He W, Xian Y, Ciobanu AO, Kamath DY, Fox KA, Rao MP, Pokorney SD, Berwanger O, Tajer C, de Barros e Silva PGM, Roettig ML, Huo Y, Granger CB; IMPACT-AF investigators. A multifaceted intervention to improve treatment with oral anticoagulants in atrial fibrillation (IMPACT-AF): an international, clusterrandomised trial. Lancet 2017;390:1737-1746.

111. Camm AJ, Luscher TF, Serruys P. The ESC Textbook of Cardiovascular Medicine, 2nd ed. Oxford, NY: Oxford University Press; 2009.

112. Germeys J, Desteghe L, Vijgen J, Dilling-Boer D, Koopman P, Schurmans J, Dendale $\mathrm{P}$, Heidbuchel $\mathrm{H}$. The effect of online targeted education on procedure-specific knowledge of atrial fibrillation patients undergoing cardioversion or ablation. Acta Cardiol 2017;72:573.

113. Shore S, Ho PM, Lambert-Kerzner A, Glorioso TJ, Carey EP, Cunningham F, Longo L, Jackevicius C, Rose A, Turakhia MP. Site-level variation in and practices associated with dabigatran adherence. JAMA 2015;313:1443-1450.

114. Guo Y, Chen Y, Lane DA, Liu L, Wang Y, Lip GYH. Mobile health technology for atrial fibrillation management integrating decision support, education, and patient involvement: MAF App Trial. Am J Med 2017;130:1388-1396.e6.
115. Santo K, Richtering SS, Chalmers J, Thiagalingam A, Chow CK, Redfern J. Mobile phone apps to improve medication adherence: a systematic stepwise process to identify high-quality apps. JMIR Mhealth Uhealth 2016;4:e132.

116. Bae JP, Dobesh PP, Klepser DG, Anderson JD, Zagar AJ, McCollam PL, Tomlin ME. Adherence and dosing frequency of common medications for cardiovascular patients. Am J Manag Care 2012;18:139-146.

117. Weeda ER, Coleman Cl, McHorney CA, Crivera C, Schein JR, Sobieraj DM Impact of once- or twice-daily dosing frequency on adherence to chronic cardiovascular disease medications: a meta-regression analysis. Int J Cardiol 2016;216:104-109.

118. Laliberte F, Nelson WW, Lefebvre P, Schein JR, Rondeau-Leclaire J, Duh MS Impact of daily dosing frequency on adherence to chronic medications among nonvalvular atrial fibrillation patients. Adv Ther 2012;29:675-690.

119. Sorensen R, Jamie Nielsen B, Langtved Pallisgaard J, Ji-Young Lee C, TorpPedersen $C$. Adherence with oral anticoagulation in non-valvular atrial fibrillation: a comparison of vitamin $\mathrm{K}$ antagonists and non-vitamin $\mathrm{K}$ antagonists. Eur Heart J Cardiovasc Pharmacother 2017;3:151-156.

120. Forslund T, Wettermark B, Hjemdahl P. Comparison of treatment persistence with different oral anticoagulants in patients with atrial fibrillation. Eur J Clin Pharmacol 2016;72:329-338.

121. Noseworthy PA, Yao X, Abraham NS, Sangaralingham LR, McBane RD, Shah ND. Direct comparison of dabigatran, rivaroxaban, and apixaban for effectiveness and safety in nonvalvular atrial fibrillation. Chest 2016;150:1302-1312.

122. Al-Khalili F, Lindström C, Benson L. The safety and persistence of non-vitamin$\mathrm{K}$-antagonist oral anticoagulants in atrial fibrillation patients treated in a well structured atrial fibrillation clinic. Curr Med Res Opin 2016;32:779-785.

123. Vrijens $B$, Heidbuchel $H$. Non-vitamin $K$ antagonist oral anticoagulants: considerations on once- vs. twice-daily regimens and their potential impact on medication adherence. Europace 2015;17:514-523.

124. Kreutz R, Persson PB, Kubitza D, Thelen K, Heitmeier S, Schwers S, Becka M, Hemmrich M. Dissociation between the pharmacokinetics and pharmacodynamics of once-daily rivaroxaban and twice-daily apixaban: a randomized crossover study. J Thromb Haemost 2017;15:2017-2028.

125. Ruff CT, Giugliano RP, Braunwald E, Mercuri M, Curt V, Betcher J, Grip L, Cange AL, Crompton AE, Murphy SA, Deenadayalu N, Antman EM. Transition of patients from blinded study drug to open-label anticoagulation: the ENGAGE AF-TIMI 48 trial. J Am Coll Cardiol 2014;64:576-584.

126. Patel MR, Hellkamp AS, Lokhnygina Y, Piccini JP, Zhang Z, Mohanty S, Singer DE, Hacke W, Breithardt G, Halperin JL, Hankey G], Becker RC, Nessel CC, Berkowitz SD, Califf RM, Fox KA, Mahaffey KW. Outcomes of discontinuing rivaroxaban compared with warfarin in patients with nonvalvular atrial fibrillation: analysis from the ROCKET AF trial (Rivaroxaban Once-Daily, Oral, Direct Factor Xa Inhibition Compared With Vitamin K Antagonism for Prevention of Stroke and Embolism Trial in Atrial Fibrillation). J Am Coll Cardiol 2013;61:651-658.

127. Granger CB, Lopes RD, Hanna M, Ansell J, Hylek EM, Alexander JH, Thomas L, Wang J, Bahit MC, Verheugt F, Lawrence J, Xavier D, Wallentin L. Clinical events after transitioning from apixaban versus warfarin to warfarin at the end of the Apixaban for Reduction in Stroke and Other Thromboembolic Events in Atrial Fibrillation (ARISTOTLE) trial. Am Heart J 2015;169:25-30.

128. Gnoth MJ, Buetehorn U, Muenster U, Schwarz T, Sandmann S. In vitro and in vivo P-glycoprotein transport characteristics of rivaroxaban. J Pharmacol Exp Ther 2011;338:372-380.

129. Mueck W, Kubitza D, Becka M. Co-administration of rivaroxaban with drugs that share its elimination pathways: pharmacokinetic effects in healthy subjects. BrJ Clin Pharmacol 2013;76:455-466.

130. Wang L, Zhang D, Raghavan N, Yao M, Ma L, Frost CA, Maxwell BD, Chen S-y, He K, Goosen TC, Griffith WH, Grossman SJ. In vitro assessment of metabolic drug-drug interaction potential of apixaban through cytochrome P450 phenotyping, inhibition, and induction studies. Drug Metab Dispos 2010;38:448-458.

131. Mueck W, Stampfuss J, Kubitza D, Becka M. Clinical pharmacokinetic and pharmacodynamic profile of rivaroxaban. Clin Pharmacokinet 2014;53:1-16.

132. Salazar DE, Mendell J, Kastrissios H, Green M, Carrothers TJ, Song S, Patel I, Bocanegra TS, Antman EM, Giugliano RP, Kunitada S, Dornseif B, Shi M, Tachibana M, Zhou S, Rohatagi S. Modelling and simulation of edoxaban exposure and response relationships in patients with atrial fibrillation. Thromb Haemost 2012;107:925-936.

133. Steffel J, Giugliano RP, Braunwald E, Murphy SA, Atar D, Heidbuchel H, Camm AJ, Antman EM, Ruff CT. Edoxaban vs. warfarin in patients with atrial fibrillation on amiodarone: a subgroup analysis of the ENGAGE AF-TIMI 48 trial. Eur Heart J 2015;36:2239-2245.

134. Mendell J, Zahir H, Matsushima N, Noveck R, Lee F, Chen S, Zhang G, Shi M. Drug-drug interaction studies of cardiovascular drugs involving P-glycoprotein, an efflux transporter, on the pharmacokinetics of edoxaban, an oral factor $\mathrm{Xa}$ inhibitor. Am J Cardiovasc Drugs 2013;13:331-342. 
135. Frost C, Song Y, Yu Z, Wang J, Lee LS, Schuster A, Pollack A, LaCreta F. The effect of apixaban on the pharmacokinetics of digoxin and atenolol in healthy subjects. Clin Pharmacol 2017;9:19-28.

136. Frost CE, Byon W, Song Y, Wang J, Schuster AE, Boyd RA, Zhang D, Yu Z, Dias C, Shenker A, LaCreta F. Effect of ketoconazole and diltiazem on the pharmacokinetics of apixaban, an oral direct factor $\mathrm{Xa}$ inhibitor. $\mathrm{Br} / \mathrm{Clin}$ Pharmacol 2015;79:838-846.

137. Mendell J, Noveck R, Zahir H, Lee F, Petrushin V, Rubets I, Zhang G, Shi M, Chen $S$. The effect of quinidine and verapamil, P glycoprotein/CYP3A4/5 inhibitors, on edoxaban pharmacokinetics and pharmacodynamics. Basic Clin Pharmacol Toxicol 2010;107:2848. (Abstract).

138. Vakkalagadda B, Frost C, Byon W, Boyd RA, Wang J, Zhang D, Yu Z, Dias C, Shenker A, LaCreta F. Effect of rifampin on the pharmacokinetics of apixaban, an oral direct inhibitor of factor Xa. Am J Cardiovasc Drugs 2016;16:119-127.

139. Frost C, Shenker A, Gandhi MD, Pursley J, Barrett YC, Wang J, Zhang D, Byon W, Boyd RA, LaCreta F. Evaluation of the effect of naproxen on the pharmacokinetics and pharmacodynamics of apixaban. $\mathrm{Br} /$ Clin Pharmacol 2014:78:877-885.

140. Kubitza D, Becka M, Zuehlsdorf M, Mueck W. Effect of food, an antacid, and the $\mathrm{H} 2$ antagonist ranitidine on the absorption of BAY 59-7939 (rivaroxaban), an oral, direct factor $\mathrm{Xa}$ inhibitor, in healthy subjects. J Clin Pharmacol 2006; 46:549-558.

141. Cannon CP, Bhatt DL, Oldgren J, Lip GYH, Ellis SG, Kimura T, Maeng M, Merkely B, Zeymer U, Gropper S, Nordaby M, Kleine E, Harper R, Manassie J, Januzzi JL, Ten Berg JM, Steg PG, Hohnloser SH. Dual antithrombotic therapy with dabigatran after PCl in atrial fibrillation. N Engl J Med 2017;377:1513-1524.

142. U.S. Food and Drug Administration. Drug Development and Drug Interactions: Table of Substrates, Inhibitors and Inducers. https://www.fda.gov/Drugs/ DevelopmentApprovalProcess/DevelopmentResources/ DruglnteractionsLabeling/ucm093664.htm (8 Febuary 2018).

143. Parasrampuria DA, Mendell J, Shi M, Matsushima N, Zahir H, Truitt K. Edoxaban drug-drug interactions with ketoconazole, erythromycin, and cyclosporine. Br J Clin Pharmacol 2016;82:1591-1600.

144. Short NJ, Connors JM. New oral anticoagulants and the cancer patient. Oncologist 2014;19:82-93.

145. Zhang C, Kwan P, Zuo Z, Baum L. The transport of antiepileptic drugs by Pglycoprotein. Adv Drug Deliv Rev 2012;64:930-942.

146. Schelleman H, Pollard JR, Newcomb C, Markowitz CE, Bilker WB, Leonard MB, Hennessy S. Exposure to CYP3A4-inducing and CYP3A4-non-inducing antiepileptic agents and the risk of fractures. Pharmacoepidemiol Drug Saf 2011;20:619-625.

147. Stollberger C, Finsterer J. Interactions between non-vitamin K oral anticoagulants and antiepileptic drugs. Epilepsy Res 2016;126:98-101.

148. Wiggins BS, Northup A, Johnson D, Senfield J. Reduced anticoagulant effect of dabigatran in a patient receiving concomitant phenytoin. Pharmacotherapy 2016;36:e5-e7.

149. Stollberger C, Finsterer J. Prolonged anticoagulant activity of rivaroxaban in a polymorbid elderly female with non-convulsive epileptic state. Heart Lung 2014:43:262-263.

150. Stollberger C. Drug interactions with new oral anticoagulants in elderly patients. Expert Rev Clin Pharmacol 2017:10:1191-1202.

151. LaHaye SA, Gibbens SL, Ball DG, Day AG, Olesen JB, Skanes AC. A clinical decision aid for the selection of antithrombotic therapy for the prevention of stroke due to atrial fibrillation. Eur Heart J 2012;33:2163-2171.

152. Reilly PA, Lehr T, Haertter S, Connolly SJ, Yusuf S, Eikelboom JW, Ezekowitz MD, Nehmiz G, Wang S, Wallentin L; RE-LY Investigators. The effect of dabigatran plasma concentrations and patient characteristics on the frequency of ischemic stroke and major bleeding in atrial fibrillation patients: the RE-LY Trial (Randomized Evaluation of Long-Term Anticoagulation Therapy). J Am Coll Cardiol 2014;63:321-328.

153. Ruff CT, Giugliano RP, Braunwald E, Morrow DA, Murphy SA, Kuder JF, Deenadayalu N, Jarolim P, Betcher J, Shi M, Brown K, Patel I, Mercuri M, Antman EM. Association between edoxaban dose, concentration, anti-Factor Xa activity, and outcomes: an analysis of data from the randomised, doubleblind ENGAGE AF-TIMI 48 trial. Lancet 2015;385:2288-2295.

154. Lip GY, Clemens A, Noack H, Ferreira J, Connolly SJ, Yusuf S. Patient outcomes using the European label for dabigatran. A post-hoc analysis from the RE-LY database. Thromb Haemost 2014;111:933-942.

155. Eikelboom JW, Wallentin L, Connolly SJ, Ezekowitz M, Healey JS, Oldgren J, Yang S, Alings M, Kaatz S, Hohnloser SH, Diener HC, Franzosi MG, Huber K, Reilly P, Varrone J, Yusuf S. Risk of bleeding with 2 doses of dabigatran compared with warfarin in older and younger patients with atrial fibrillation: an analysis of the randomized evaluation of long-term anticoagulant therapy (RE-LY) trial. Circulation 2011;123:2363-2372

156. Hori M, Matsumoto M, Tanahashi N, Momomura S, Uchiyama S, Goto S, Izumi T, Koretsune Y, Kajikawa M, Kato M, Ueda H, Iwamoto K, Tajiri M; J-ROCKET
AF study investigators. Rivaroxaban vs. warfarin in Japanese patients with atrial fibrillation - the J-ROCKET AF study. Circ J 2012;76:2104-2111.

157. Alexander JH, Andersson U, Lopes RD, Hijazi Z, Hohnloser SH, Ezekowitz JA, Halvorsen S, Hanna M, Commerford P, Ruzyllo W, Huber K, Al-Khatib SM, Granger CB, Wallentin L; Apixaban for Reduction of Stroke and Other Thromboembolic Complications in Atrial Fibrillation (ARISTOTLE) Investigators. Apixaban $5 \mathrm{mg}$ twice daily and clinical outcomes in patients with atrial fibrillation and advanced age, low body weight, or high creatinine: a secondary analysis of a randomized clinical trial. JAMA Cardiol 2016;1:673-681.

158. Blech S, Ebner T, Ludwig-Schwellinger E, Stangier J, Roth W. The metabolism and disposition of the oral direct thrombin inhibitor, dabigatran, in humans. Drug Metab Dispos 2008;36:386-399.

159. Stangier J, Stahle H, Rathgen K, Fuhr R. Pharmacokinetics and pharmacodynamics of the direct oral thrombin inhibitor dabigatran in healthy elderly subjects. Clinical Pharmacokinetics 2008;47:47-59.

160. Mendell J, Tachibana M, Shi M, Kunitada S. Effects of food on the pharmacokinetics of edoxaban, an oral direct factor $X_{a}$ inhibitor, in healthy volunteers. J Clin Pharmacol 2011;51:687-694.

161. Upreti VV, Song Y, Wang J, Byon W, Boyd RA, Pursley JM, Lacreta F, Frost CE. Effect of famotidine on the pharmacokinetics of apixaban, an oral direct factor Xa inhibitor. Clin Pharmacol 2013;5:59-66.

162. Song Y, Chang M, Suzuki A, Frost RJ, Kelly A, LaCreta F, Frost C. Evaluation of crushed tablet for oral administration and the effect of food on apixaban pharmacokinetics in healthy adults. Clin Ther 2016;38:1674-1685.e1.

163. Duchin K, Duggal A, Atiee GJ, Kidokoro M, Takatani T, Shipitofsky NL, He L, Zhang G, Kakkar T. An open-label crossover study of the pharmacokinetics of the 60-mg edoxaban tablet crushed and administered either by a nasogastric tube or in apple puree in healthy adults. Clin Pharmacokinet 2018;57:221-228.

164. Moore KT, Krook MA, Vaidyanathan S, Sarich TC, Damaraju CV, Fields LE. Rivaroxaban crushed tablet suspension characteristics and relative bioavailability in healthy adults when administered orally or via nasogastric tube. Clin Pharmacol Drug Dev 2014;3:321-327.

165. Song Y, Wang X, Perlstein I, Wang J, Badawy S, Frost C, LaCreta F. Relative bioavailability of apixaban solution or crushed tablet formulations administered by mouth or nasogastric tube in healthy subjects. Clin Ther 2015;37:1703-1712.

166. Liesenfeld KH, Lehr T, Dansirikul C, Reilly PA, Connolly SJ, Ezekowitz MD, Yusuf S, Wallentin L, Haertter S, Staab A. Population pharmacokinetic analysis of the oral thrombin inhibitor dabigatran etexilate in patients with non-valvular atrial fibrillation from the RE-LY trial. J Thromb Haemost 2011;9:2168-2175.

167. Ruff CT, Giugliano RP, Antman EM, Crugnale SE, Bocanegra T, Mercuri M, Hanyok J, Patel I, Shi M, Salazar D, McCabe CH, Braunwald E. Evaluation of the novel factor $\mathrm{Xa}$ inhibitor edoxaban compared with warfarin in patients with atrial fibrillation: design and rationale for the Effective aNticoaGulation with factor $\mathrm{XA}$ next GEneration in Atrial Fibrillation-Thrombolysis In Myocardial Infarction study 48 (ENGAGE AF-TIMI 48). Am Heart J 2010;160:635-641.

168. Godier A, Dincq AS, Martin AC, Radu A, Leblanc I, Antona M, Vasse M, Golmard JL, Mullier F, Gouin-Thibault I. Predictors of pre-procedural concentrations of direct oral anticoagulants: a prospective multicentre study. Eur Heart | 2017;38:2431-2439.

169. Ruschitzka F, Meier PJ, Turina M, Luscher TF, Noll G. Acute heart transplant rejection due to Saint John's wort. Lancet 2000;355:548-549.

170. Dans AL, Connolly SJ, Wallentin L, Yang S, Nakamya J, Brueckmann M, Ezekowitz M, Oldgren J, Eikelboom JW, Reilly PA, Yusuf S. Concomitant use of antiplatelet therapy with dabigatran or warfarin in the Randomized Evaluation of Long-Term Anticoagulation Therapy (RE-LY) trial. Circulation 2013;127:634-640.

171. Mega JL, Braunwald E, Wiviott SD, Bassand JP, Bhatt DL, Bode C, Burton P, Cohen M, Cook-Bruns N, Fox KA, Goto S, Murphy SA, Plotnikov AN, Schneider D, Sun X, Verheugt FW, Gibson CM; ATLAS ACS 2-TIMI 51 Investigators. Rivaroxaban in patients with a recent acute coronary syndrome. N EnglJ Med 2012;366:9-19.

172. Alexander JH, Becker RC, Bhatt DL, Cools F, Crea F, Dellborg M, Fox KA, Goodman SG, Harrington RA, Huber K, Husted S, Lewis BS, Lopez-Sendon J, Mohan P, Montalescot G, Ruda M, Ruzyllo W, Verheugt F, Wallentin L. Apixaban, an oral, direct, selective factor $X a$ inhibitor, in combination with antiplatelet therapy after acute coronary syndrome: results of the Apixaban for Prevention of Acute Ischemic and Safety Events (APPRAISE) trial. Circulation 2009;119:2877-2885.

173. Proietti M, Raparelli V, Olshansky B, Lip GY. Polypharmacy and major adverse events in atrial fibrillation: observations from the AFFIRM trial. Clin Res Cardiol 2016; 105:412-420.

174. Piccini JP, Hellkamp AS, Washam JB, Becker RC, Breithardt G, Berkowitz SD, Halperin JL, Hankey GJ, Hacke W, Mahaffey KW, Nessel CC, Singer DE, Fox KA, Patel MR. Polypharmacy and the efficacy and safety of rivaroxaban versus warfarin in the prevention of stroke in patients with nonvalvular atrial fibrillation. Circulation 2016:133:352-360. 
175. Focks J, Brouwer MA, Wojdyla DM, Thomas L, Lopes RD, Washam JB, Lanas F, Xavier D, Husted S, Wallentin L, Alexander JH, Granger CB, Verheugt FW. Polypharmacy and effects of apixaban versus warfarin in patients with atrial fibrillation: post hoc analysis of the ARISTOTLE trial. Bmj 2016;353:i2868.

176. Bansal N, Zelnick LR, Alonso A, Benjamin EJ, de Boer IH, Deo R, Katz R, Kestenbaum B, Mathew J, Robinson-Cohen C, Sarnak MJ, Shlipak MG, Sotoodehnia N, Young B, Heckbert SR. eGFR and albuminuria in relation to risk of incident atrial fibrillation: a meta-analysis of the Jackson Heart Study, the Multi-Ethnic Study of Atherosclerosis, and the Cardiovascular Health Study. Clin J Am Soc Nephrol 2017;12:1386-1398.

177. Go AS, Fang MC, Udaltsova N, Chang Y, Pomernacki NK, Borowsky L, Singer $\mathrm{DE}$. Impact of proteinuria and glomerular filtration rate on risk of thromboembolism in atrial fibrillation: the anticoagulation and risk factors in atrial fibrillation (ATRIA) study. Circulation 2009;119:1363-1369.

178. Soliman EZ, Prineas RJ, Go AS, Xie D, Lash JP, Rahman M, Ojo A, Teal VL, Jensvold NG, Robinson NL, Dries DL, Bazzano L, Mohler ER, Wright JT, Feldman HI. Chronic kidney disease and prevalent atrial fibrillation: the Chronic Renal Insufficiency Cohort (CRIC). Am Heart J 2010;159:1102-1107.

179. Watanabe H, Watanabe T, Sasaki S, Nagai K, Roden DM, Aizawa Y. Close bidirectional relationship between chronic kidney disease and atrial fibrillation: the Niigata preventive medicine study. Am Heart J 2009;158:629-636.

180. Reinecke H, Brand E, Mesters R, Schabitz WR, Fisher M, Pavenstadt H, Breithardt $G$. Dilemmas in the management of atrial fibrillation in chronic kidney disease. J Am Soc Nephrol 2009;20:705-711.

181. Steffel J, Hindricks G. Apixaban in renal insufficiency: successful navigation between the Scylla and Charybdis. Eur Heart J 2012;33:2766-2768.

182. Stangier J, Rathgen K, Stahle H, Gansser D, Roth W. The pharmacokinetics, pharmacodynamics and tolerability of dabigatran etexilate, a new oral direct thrombin inhibitor, in healthy male subjects. Br J Clin Pharmacol 2007;64:292-303.

183. Raghavan N, Frost CE, Yu Z, He K, Zhang H, Humphreys WG, Pinto D, Chen S, Bonacorsi S, Wong PC, Zhang D. Apixaban metabolism and pharmacokinetics after oral administration to humans. Drug Metab Dispos 2009;37:74-81.

184. Ogata K, Mendell-Harary J, Tachibana M, Masumoto H, Oguma T, Kojima M, Kunitada S. Clinical safety, tolerability, pharmacokinetics, and pharmacodynamics of the novel factor $X_{a}$ inhibitor edoxaban in healthy volunteers. J Clin Pharmacol 2010;50:743-753.

185. Mueck W, Lensing AW, Agnelli G, Decousus H, Prandoni P, Misselwitz F. Rivaroxaban: population pharmacokinetic analyses in patients treated for acute deep-vein thrombosis and exposure simulations in patients with atrial fibrillation treated for stroke prevention. Clin Pharmacokinet 2011;50:675-686.

186. Kubitza D, Becka M, Voith B, Zuehlsdorf M, Wensing G. Safety, pharmacodynamics, and pharmacokinetics of single doses of BAY 59-7939, an oral, direct factor Xa inhibitor. Clin Pharmacol Ther 2005;78:412-421.

187. Levey AS, Stevens LA, Schmid CH, Zhang YL, Castro AF, Feldman HI 3rd, Kusek JW, Eggers P, Van Lente F, Greene T, Coresh J; CKD-EPI (Chronic Kidney Disease Epidemiology Collaboration). A new equation to estimate glomerular filtration rate. Ann Intern Med 2009;150:604-612.

188. Lindner SM, Fordyce CB, Hellkamp AS, Lokhnygina Y, Piccini JP, Breithardt G, Mahaffey KW, Singer DE, Hacke W, Halperin JL, Hankey GJ, Berkowitz SD, Nessel CC, Becker RC, Fox KA, Patel MR; ROCKET AF Steering Committee and Investigators. Treatment consistency across levels of baseline renal function with rivaroxaban or warfarin: a ROCKET AF (Rivaroxaban Once-Daily, Oral, Direct Factor $\mathrm{Xa}$ Inhibition Compared With Vitamin $\mathrm{K}$ Antagonism for Prevention of Stroke and Embolism Trial in Atrial Fibrillation) Analysis. Circulation 2017;135:1001-1003.

189. Fanikos J, Burnett AE, Mahan CE, Dobesh PP. Renal function considerations for stroke prevention in atrial fibrillation. Am J Med 2017;130:1015-1023.

190. Bohula EA, Giugliano RP, Ruff CT, Kuder JF, Murphy SA, Antman EM, Braunwald E. Impact of renal function on outcomes with edoxaban in the ENGAGE AF-TIMI 48 Trial. Circulation 2016;134:24-36.

191. Hart RG, Pearce LA, Asinger RW, Herzog CA. Warfarin in atrial fibrillation patients with moderate chronic kidney disease. Clin J Am Soc Nephrol 2011;6:2599-2604.

192. Olesen JB, Lip GY, Kamper AL, Hommel K, Kober L, Lane DA, Lindhardsen J, Gislason GH, Torp-Pedersen C. Stroke and bleeding in atrial fibrillation with chronic kidney disease. N Engl J Med 2012;367:625-635.

193. Bonde AN, Lip GY, Kamper AL, Hansen PR, Lamberts M, Hommel K, Hansen ML, Gislason GH, Torp-Pedersen C, Olesen JB. Net clinical benefit of antithrombotic therapy in patients with atrial fibrillation and chronic kidney disease: a nationwide observational cohort study. J Am Coll Cardiol 2014;64:2471-2482.

194. Friberg L, Benson L, Lip GY. Balancing stroke and bleeding risks in patients with atrial fibrillation and renal failure: the Swedish Atrial Fibrillation Cohort study. Eur Heart J 2015;36:297-306.

195. Hijazi Z, Hohnloser SH, Oldgren J, Andersson U, Connolly SJ, Eikelboom JW, Ezekowitz MD, Reilly PA, Siegbahn A, Yusuf S, Wallentin L. Efficacy and safety of dabigatran compared with warfarin in relation to baseline renal function in patients with atrial fibrillation: a RE-LY (Randomized Evaluation of Long-term Anticoagulation Therapy) trial analysis. Circulation 2014;129:961-970.

196. Fox KA, Piccini JP, Wojdyla D, Becker RC, Halperin JL, Nessel CC, Paolini JF, Hankey G], Mahaffey KW, Patel MR, Singer DE, Califf RM. Prevention of stroke and systemic embolism with rivaroxaban compared with warfarin in patients with non-valvular atrial fibrillation and moderate renal impairment. Eur Heart J 2011;32:2387-2394.

197. Hohnloser SH, Hijazi Z, Thomas L, Alexander JH, Amerena J, Hanna M, Keltai M, Lanas F, Lopes RD, Lopez-Sendon J, Granger CB, Wallentin L. Efficacy of apixaban when compared with warfarin in relation to renal function in patients with atrial fibrillation: insights from the ARISTOTLE trial. Eur Heart 2012;33:2821-2830.

198. Hijazi Z, Hohnloser SH, Andersson U, Alexander JH, Hanna M, Keltai M, Parkhomenko A, Lopez-Sendon JL, Lopes RD, Siegbahn A, Granger CB, Wallentin L. Efficacy and safety of apixaban compared with warfarin in patients with atrial fibrillation in relation to renal function over time: insights from the ARISTOTLE Randomized Clinical Trial. JAMA Cardiol 2016;1:451-460.

199. Fordyce CB, Hellkamp AS, Lokhnygina Y, Lindner SM, Piccini JP, Becker RC, Berkowitz SD, Breithardt G, Fox KA, Mahaffey KW, Nessel CC, Singer DE, Patel MR. On-treatment outcomes in patients with worsening renal function with rivaroxaban compared with warfarin: insights from ROCKET AF. Circulation 2016;134:37-47.

200. Bohm M, Ezekowitz MD, Connolly SJ, Eikelboom JW, Hohnloser SH, Reilly PA Schumacher H, Brueckmann M, Schirmer SH, Kratz MT, Yusuf S, Diener HC, Hijazi Z, Wallentin L. Changes in renal function in patients with atrial fibrillation: an analysis from the RE-LY Trial. J Am Coll Cardiol 2015;65:2481-2493.

201. Brodsky SV, Nadasdy T, Rovin BH, Satoskar AA, Nadasdy GM, Wu HM, Bhatt UY, Hebert LA. Warfarin-related nephropathy occurs in patients with and without chronic kidney disease and is associated with an increased mortality rate. Kidney Int 2011;80:181-189.

202. Yao X, Shah ND, Sangaralingham LR, Gersh B], Noseworthy PA. Non-vitamin K antagonist oral anticoagulant dosing in patients with atrial fibrillation and renal dysfunction. J Am Coll Cardiol 2017;69:2779-2790.

203. Shah M, Avgil Tsadok M, Jackevicius CA, Essebag V, Eisenberg MJ, Rahme E, Humphries KH, Tu JV, Behlouli H, Guo H, Pilote L. Warfarin use and the risk for stroke and bleeding in patients with atrial fibrillation undergoing dialysis. Circulation 2014;129:1196-1203.

204. Galloway PA, El-Damanawi R, Bardsley V, Pritchard NR, Fry AC, Ojha SK, Hiemstra TF. Vitamin $\mathrm{K}$ antagonists predispose to calciphylaxis in patients with end-stage renal disease. Nephron 2015;129:197-201.

205. Hayashi M, Takamatsu I, Kanno Y, Yoshida T, Abe T, Sato Y; Japanese Calciphylaxis Study Group. A case-control study of calciphylaxis in Japanese end-stage renal disease patients. Nephrol Dial Transplant 2012;27:1580-1584.

206. Han KH, O'Neill WC. Increased peripheral arterial calcification in patients receiving warfarin. J Am Heart Assoc 2016;5:e002665.

207. Wilmer WA, Magro CM. Calciphylaxis: emerging concepts in prevention, diagnosis, and treatment. Semin Dial 2002;15:172-186.

208. Herzog CA, Asinger RW, Berger AK, Charytan DM, Diez J, Hart RG, Eckardt KU, Kasiske BL, McCullough PA, Passman RS, DeLoach SS, Pun PH, Ritz E. Cardiovascular disease in chronic kidney disease. A clinical update from Kidney Disease: improving Global Outcomes (KDIGO). Kidney Int 2011;80: 572-586.

209. Chan KE, Edelman ER, Wenger JB, Thadhani RI, Maddux FW. Dabigatran and rivaroxaban use in atrial fibrillation patients on hemodialysis. Circulation 2015;131:972-979.

210. Mavrakanas TA, Samer CF, Nessim SJ, Frisch G, Lipman ML. Apixaban pharmacokinetics at steady state in hemodialysis patients. I Am Soc Nephrol 2017; 28:2241-2248.

211. Koretsune Y, Yamashita T, Kimura T, Fukuzawa M, Abe K, Yasaka M. Shortterm safety and plasma concentrations of edoxaban in Japanese patients with non-valvular atrial fibrillation and severe renal impairment. Circ J 2015;79:1486-1495.

212. De Vriese AS, Caluwe R, Bailleul E, De Bacquer D, Borrey D, Van Vlem B, Vandecasteele SJ, Emmerechts J. Dose-finding study of rivaroxaban in hemodialysis patients. Am J Kidney Dis 2015;66:91-98.

213. Reinecke H, Engelbertz C, Schabitz WR. Preventing stroke in patients with chronic kidney disease and atrial fibrillation: benefit and risks of old and new oral anticoagulants. Stroke 2013;44:2935-2941.

214. Khoury T, Ayman AR, Cohen J, Daher S, Shmuel C, Mizrahi M. The complex role of anticoagulation in cirrhosis: an updated review of where we are and where we are going. Digestion 2016;93:149-159.

215. Lauschke VM, Ingelman-Sundberg M. The importance of patient-specific factors for hepatic drug response and toxicity. Int J Mol Sci 2016;17:1714.

216. Efird LM, Mishkin DS, Berlowitz DR, Ash AS, Hylek EM, Ozonoff A, Reisman JI, Zhao S, Jasuja GK, Rose AJ. Stratifying the risks of oral anticoagulation in patients with liver disease. Circ Cardiovasc Qual Outcomes 2014;7:461-467. 
217. Kubitza D, Roth A, Becka M, Alatrach A, Halabi A, Hinrichsen H, Mueck W. Effect of hepatic impairment on the pharmacokinetics and pharmacodynamics of a single dose of rivaroxaban, an oral, direct Factor $\mathrm{Xa}$ inhibitor. $\mathrm{Br}$ J Clin Pharmacol 2013;76:89-98.

218. Intagliata NM, Henry ZH, Maitland H, Shah NL, Argo CK, Northup PG, Caldwell SH. Direct oral anticoagulants in cirrhosis patients pose similar risks of bleeding when compared to traditional anticoagulation. Dig Dis Sci 2016;61:1721-1727.

219. Hum J, Shatzel JJ, Jou JH, Deloughery TG. The efficacy and safety of direct oral anticoagulants vs traditional anticoagulants in cirrhosis. Eur J Haematol 2017;98:393-397.

220. Keisu M, Andersson TB. Drug-induced liver injury in humans: the case of ximelagatran. Handb Exp Pharmacol 2010;196:407-418.

221. Caldeira D, Barra M, Santos AT, de Abreu D, Pinto FJ, Ferreira JJ, Costa J. Risk of drug-induced liver injury with the new oral anticoagulants: systematic review and meta-analysis. Heart 2014;100:550-556.

222. Alonso A, MacLehose RF, Chen LY, Bengtson LG, Chamberlain AM, Norby FL, Lutsey PL. Prospective study of oral anticoagulants and risk of liver injury in patients with atrial fibrillation. Heart 2017;103:834-839.

223. Potpara TS, Lip GY. Drug-induced liver injury with oral anticoagulants: a threat or not? Heart 2017;103:809-811.

224. Licata A, Puccia F, Lombardo V, Serruto A, Minissale MG, Morreale I, Giannitrapani L, Soresi M, Montalto G, Almasio PL. Rivaroxaban-induced hepatotoxicity: review of the literature and report of new cases. Eur J Gastroenterol Hepatol 2018;30:226-232.

225. Douxfils J, Ageno W, Samama CM, Lessire S, Ten Cate H, Verhamme P, Dogne JM, Mullier F. Laboratory testing in patients treated with direct oral anticoagulants: a practical guide for clinicians. J Thromb Haemost 2018;16:209-219.

226. Douxfils J, Mullier F, Loosen C, Chatelain C, Chatelain B, Dogne JM. Assessment of the impact of rivaroxaban on coagulation assays: laboratory recommendations for the monitoring of rivaroxaban and review of the literature. Thromb Res 2012;130:956-966.

227. Douxfils J, Mullier F, Robert S, Chatelain C, Chatelain B, Dogne JM. Impact of dabigatran on a large panel of routine or specific coagulation assays. Laboratory recommendations for monitoring of dabigatran etexilate. Thromb Haemost 2012;107:985-997.

228. van Ryn J, Baruch L, Clemens A. Interpretation of point-of-care INR results in patients treated with dabigatran. Am J Med 2012;125:417-420.

229. van Ryn J, Stangier J, Haertter S, Liesenfeld KH, Wienen W, Feuring M, Clemens A. Dabigatran etexilate-a novel, reversible, oral direct thrombin inhibitor: interpretation of coagulation assays and reversal of anticoagulant activity. Thromb Haemost 2010;103:1116-1127.

230. Ezekowitz MD, Reilly PA, Nehmiz G, Simmers TA, Nagarakanti R, ParchamAzad K, Pedersen KE, Lionetti DA, Stangier J, Wallentin L. Dabigatran with or without concomitant aspirin compared with warfarin alone in patients with nonvalvular atrial fibrillation (PETRO Study). Am J Cardiol 2007;100:1419-1426.

231. Douxfils J, Chatelain C, Chatelain B, Dogne JM, Mullier F. Impact of apixaban on routine and specific coagulation assays: a practical laboratory guide. Thromb Haemost 2013;110:283-294.

232. Cuker A, Husseinzadeh $\mathrm{H}$. Laboratory measurement of the anticoagulant activity of edoxaban: a systematic review. J Thromb Thrombolysis 2015;39:288-294.

233. Lindhoff-Last E, Samama MM, Ortel TL, Weitz JI, Spiro TE. Assays for measuring rivaroxaban: their suitability and limitations. Ther Drug Monit 2010;32:673-679.

234. Mani H, Herth N, Kasper A, Wendt T, Schuettfort G, Weil Y, Pfeilschifter W, Linnemann B, Herrmann E, Lindhoff-Last E. Point-of-care coagulation testing for assessment of the pharmacodynamic anticoagulant effect of direct oral anticoagulant. Ther Drug Monit 2014;36:624-631.

235. Kaess BM, Ammar S, Reents T, Dillier R, Lennerz C, Semmler V, Grebmer C, Bourier F, Buiatti A, Kolb C, Deisenhofer I, Hessling G. Comparison of safety of left atrial catheter ablation procedures for atrial arrhythmias under continuous anticoagulation with apixaban versus phenprocoumon. Am J Cardiol 2015;115:47-51.

236. Auer J, Huber K, Granger CB. Interruption of non-vitamin K antagonist anticoagulants in patients undergoing planned invasive procedures: how long is long enough? Eur Heart / 2017;38:2440-2443.

237. Kubitza D, Becka M, Roth A, Mueck W. Dose-escalation study of the pharmacokinetics and pharmacodynamics of rivaroxaban in healthy elderly subjects. Curr Med Res Opin 2008;24:2757-2765.

238. Huisman MV, Lip GY, Diener HC, Brueckmann M, van Ryn J, Clemens A Dabigatran etexilate for stroke prevention in patients with atrial fibrillation: resolving uncertainties in routine practice. Thromb Haemost 2012;107:838-847.

239. Green R, Grierson R, Sitar DS, Tenenbein M. How long after drug ingestion is activated charcoal still effective? J Toxicol Clin Toxicol 2001;39:601-605.

240. Hylek EM, Held C, Alexander JH, Lopes RD, De Caterina R, Wojdyla DM, Huber K, Jansky P, Steg PG, Hanna M, Thomas L, Wallentin L, Granger CB. Major bleeding in patients with atrial fibrillation receiving apixaban or warfarin: the ARISTOTLE Trial (Apixaban for Reduction in Stroke and Other Thromboembolic Events in Atrial Fibrillation): Predictors, Characteristics, and Clinical Outcomes. J Am Coll Cardiol 2014;63:2141-2147.

241. Peetermans M, Pollack $C$ Jr, Reilly P, Liesenborghs L, Jacquemin M, Levy JH, Weitz JI, Verhamme P. Idarucizumab for dabigatran overdose. Clin Toxicol (Phila) 2016;54:644-646

242. Piccini JP, Garg J, Patel MR, Lokhnygina Y, Goodman SG, Becker RC, Berkowitz SD, Breithardt G, Hacke W, Halperin JL, Hankey GJ, Nessel CC, Mahaffey KW Singer DE, Califf RM, Fox KA; ROCKET AF Investigators. Management of major bleeding events in patients treated with rivaroxaban vs. warfarin: results from the ROCKET AF trial. Eur Heart J 2014;35:1873-1880.

243. Majeed A, Hwang HG, Connolly SJ, Eikelboom JW, Ezekowitz MD, Wallentin L, Brueckmann M, Fraessdorf M, Yusuf S, Schulman S. Management and outcomes of major bleeding during treatment with dabigatran or warfarin. Circulation 2013;128:2325-2332

244. Giugliano RP, Ruff CT, Wiviott SD, Nordio F, Murphy SA, Kappelhof JA, Shi M, Mercuri MF, Antman EM, Braunwald E. Mortality in patients with atrial fibrillation randomized to edoxaban or warfarin: insights from the ENGAGE AF-TIMI 48 Trial. Am J Med 2016;129:850-857.e2.

245. Kawabori M, Niiya Y, Iwasaki M, Mabuchi S, Ozaki H, Matsubara K, Houkin K. Characteristics of symptomatic intracerebral hemorrhage in patient receiving direct oral anticoagulants: comparison with warfarin. I Stroke Cerebrovasc Dis 2018; [Epub ahead of print].

246. Ruff CT, Giugliano RP, Braunwald E, Hoffman EB, Deenadayalu N, Ezekowitz MD, Camm AJ, Weitz JI, Lewis BS, Parkhomenko A, Yamashita T, Antman EM. Comparison of the efficacy and safety of new oral anticoagulants with warfarin in patients with atrial fibrillation: a meta-analysis of randomised trials. Lancet 2014;383:955-962.

247. Tomaselli GF, Mahaffey KW, Cuker A, Dobesh PP, Doherty JU, Eikelboom JW, Florido R, Hucker W, Mehran R, Messe SR, Pollack CV Jr, Rodriguez F, Sarode R, Siegal D, Wiggins BS. 2017 ACC expert consensus decision pathway on management of bleeding in patients on oral anticoagulants: a report of the American College of Cardiology Task Force on Expert Consensus Decision Pathways. J Am Coll Cardiol 2017;70:3042-3067.

248. Pollack CV Jr, Reilly PA, van Ryn J, Eikelboom JW, Glund S, Bernstein RA Dubiel R, Huisman MV, Hylek EM, Kam CW, Kamphuisen PW, Kreuzer J, Levy JH, Royle G, Sellke FW, Stangier J, Steiner T, Verhamme P, Wang B, Young L, Weitz Jl. Idarucizumab for dabigatran reversal-full cohort analysis. N Engl J Med 2017;377:431-441.

249. Connolly SJ, Milling TJ, Eikelboom JW, Gibson CM, Curnutte JT, Gold A, Bronson MD, Lu G, Conley PB, Verhamme P, Schmidt J, Middeldorp S, Cohen AT, Beyer-Westendorf J, Albaladejo P, Lopez-Sendon J, Goodman S, Leeds J, Wiens BL, Siegal DM, Zotova E, Meeks B, Nakamya J, Lim WT, Crowther M ANNEXA-4 Investigators. Andexanet Alfa for acute major bleeding associated with factor Xa inhibitors. N Engl J Med 2016;375:1131-1141.

250. Ansell JE, Bakhru SH, Laulicht BE, Steiner SS, Grosso M, Brown K, Dishy V, Noveck RJ, Costin JC. Use of PER977 to reverse the anticoagulant effect of edoxaban. N Engl J Med 2014;371:2141-2142.

251. Beyer-Westendorf J, Forster K, Pannach S, Ebertz F, Gelbricht V, Thieme C Michalski F, Kohler C, Werth S, Sahin K, Tittl L, Hansel U, Weiss N. Rates, management, and outcome of rivaroxaban bleeding in daily care: results from the Dresden NOAC registry. Blood 2014;124:955-962.

252. Levi M, Eerenberg E, Kamphuisen PW. Bleeding risk and reversal strategies for old and new anticoagulants and antiplatelet agents. J Thromb Haemost 2011;9:1705-1712.

253. Stangier J, Rathgen K, Stahle H, Mazur D. Influence of renal impairment on the pharmacokinetics and pharmacodynamics of oral dabigatran etexilate: an openlabel, parallel-group, single-centre study. Clin Pharmacokinet 2010;49:259-268.

254. Getta B, Muller N, Motum P, Hsu D, Zebeljan D, Rosenfeld D. Intermittent haemodialysis and continuous veno-venous dialysis are effective in mitigating major bleeding due to dabigatran. Br J Haematol 2015;169:603-604.

255. Parasrampuria DA, Marbury T, Matsushima N, Chen S, Wickremasingha PK, He L, Dishy V, Brown KS. Pharmacokinetics, safety, and tolerability of edoxaban in end-stage renal disease subjects undergoing haemodialysis. Thromb Haemost 2015;113:719-727.

256. Wang X, Tirucherai G, Marbury TC, Wang J, Chang M, Zhang D, Song Y, Pursley J, Boyd RA, Frost C. Pharmacokinetics, pharmacodynamics, and safety of apixaban in subjects with end-stage renal disease on hemodialysis. J Clin Pharmacol 2016;56:628-636.

257. Shakur H, Roberts I, Bautista R, Caballero J, Coats T, Dewan Y, El-Sayed H, Gogichaishvili T, Gupta S, Herrera J, Hunt B, Iribhogbe P, Izurieta M, Khamis H, Komolafe E, Marrero MA, Mejia-Mantilla J, Miranda J, Morales C, Olaomi O, Olldashi F, Perel P, Peto R, Ramana PV, Ravi RR, Yutthakasemsunt S. Effects of tranexamic acid on death, vascular occlusive events, and blood transfusion in trauma patients with significant haemorrhage (CRASH-2): a randomised, placebo-controlled trial. Lancet 2010;376:23-32. 
258. Poeran J, Rasul R, Suzuki S, Danninger T, Mazumdar M, Opperer M, Boettner F, Memtsoudis SG. Tranexamic acid use and postoperative outcomes in patients undergoing total hip or knee arthroplasty in the United States: retrospective analysis of effectiveness and safety. BMJ 2014;349:g4829.

259. Enriquez A, Lip GY, Baranchuk A. Anticoagulation reversal in the era of the non-vitamin K oral anticoagulants. Europace 2016;18:955-964.

260. Healey JS, Eikelboom J, Douketis J, Wallentin L, Oldgren J, Yang S, Themeles E, Heidbuchel H, Heidbuchle H, Avezum A, Reilly P, Connolly SJ, Yusuf S, Ezekowitz M. Periprocedural bleeding and thromboembolic events with dabigatran compared with warfarin: results from the Randomized Evaluation of LongTerm Anticoagulation Therapy (RE-LY) randomized trial. Circulation 2012;126:343-348.

261. Zhou W, Schwarting S, Illanes S, Liesz A, Middelhoff M, Zorn M, Bendszus M, Heiland S, van Ryn J, Veltkamp R. Hemostatic therapy in experimental intracerebral hemorrhage associated with the direct thrombin inhibitor dabigatran. Stroke 2011;42:3594-3599.

262. Pragst I, Zeitler SH, Doerr B, Kaspereit FJ, Herzog E, Dickneite G, van Ryn J. Reversal of dabigatran anticoagulation by prothrombin complex concentrate (Beriplex P/N) in a rabbit model. J Thromb Haemost 2012;10:1841-1848.

263. Godier A, Miclot A, Le Bonniec B, Durand M, Fischer AM, Emmerich J, Marchand-Leroux C, Lecompte T, Samama CM. Evaluation of prothrombin complex concentrate and recombinant activated factor VII to reverse rivaroxaban in a rabbit model. Anesthesiology 2012;116:94-102.

264. Zahir H, Brown KS, Vandell AG, Desai M, Maa JF, Dishy V, Lomeli B, Feussner A, Feng W, He L, Grosso MA, Lanz HJ, Antman EM. Edoxaban effects on bleeding following punch biopsy and reversal by a 4-factor prothrombin complex concentrate. Circulation 2015;131:82-90.

265. Eerenberg ES, Kamphuisen PW, Sijpkens MK, Meijers JC, Buller HR, Levi M. Reversal of rivaroxaban and dabigatran by prothrombin complex concentrate: a randomized, placebo-controlled, crossover study in healthy subjects. Circulation 2011;124:1573-1579.

266. Song Y, Wang Z, Perlstein I, Wang J, LaCreta F, Frost RJA, Frost C. Reversal of apixaban anticoagulation by four-factor prothrombin complex concentrates in healthy subjects: a randomized three-period crossover study. J Thromb Haemost 2017; 15:2125-2137.

267. Levi M, Moore KT, Castillejos CF, Kubitza D, Berkowitz SD, Goldhaber SZ, Raghoebar M, Patel MR, Weitz Jl, Levy JH. Comparison of three-factor and four-factor prothrombin complex concentrates regarding reversal of the anticoagulant effects of rivaroxaban in healthy volunteers. J Thromb Haemost 2014;12:1428-1436.

268. Albaladejo P, Samama C-M, Sié P, Kauffmann S, Mémier V, Suchon P, Viallon A, David JS, Gruel Y, Bellamy L, de Maistre E, Romegoux P, Thoret S, Pernod G, Bosson J-L; GIHP-NACO Study Group. Management of severe bleeding in patients treated with direct oral anticoagulants: an observational registry analysis. Anesthesiology 2017;127:111-120.

269. Majeed A, Agren A, Holmstrom M, Bruzelius M, Chaireti R, Odeberg J, Hempel EL, Magnusson M, Frisk T, Schulman S. Management of rivaroxaban- or apixaban-associated major bleeding with prothrombin complex concentrates: a cohort study. Blood 2017;130:1706-1712.

270. Warkentin TE, Margetts P, Connolly SJ, Lamy A, Ricci C, Eikelboom JW. Recombinant factor $\mathrm{VIla}(\mathrm{rFVlla})$ and hemodialysis to manage massive dabigatran-associated postcardiac surgery bleeding. Blood 2012;119:2172-2174.

271. Douketis JD, Spyropoulos AC, Anderson JM, Arnold DM, Bates SM, Blostein M, Carrier M, Caprini JA, Clark NP, Coppens M, Dentali F, Duncan J, Gross PL, Kassis J, Kowalski S, Lee AY, Le Gal G, Le Templier G, Li N, MacKay E, Shah V, Shivakumar S, Solymoss S, Spencer FA, Syed S, Tafur AJ, Vanassche T, Thiele T, Wu C, Yeo E, Schulman S. The Perioperative Anticoagulant Use for Surgery Evaluation (PAUSE) study for patients on a direct oral anticoagulant who need an elective surgery or procedure: design and rationale. Thromb Haemost 2017;117:2415-2424

272. Beyer-Westendorf J, Gelbricht V, Forster K, Ebertz F, Kohler C, Werth S, Kuhlisch E, Stange T, Thieme C, Daschkow K, Weiss N. Peri-interventional management of novel oral anticoagulants in daily care: results from the prospective Dresden NOAC registry. Eur Heart J 2014;35:1888-1896.

273. Douketis JD, Spyropoulos AC, Kaatz S, Becker RC, Caprini JA, Dunn AS, Garcia DA, Jacobson A, Jaffer AK, Kong DF, Schulman S, Turpie AG, Hasselblad $\mathrm{V}$, Ortel TL. Perioperative bridging anticoagulation in patients with atrial fibrillation. N Engl J Med 2015;373:823-833.

274. Sivolella S, De Biagi M, Brunello G, Berengo M, Pengo V. Managing dentoalveolar surgical procedures in patients taking new oral anticoagulants. Odontology 2015; 103:258-263.

275. Johnston S. An evidence summary of the management of patients taking direct oral anticoagulants (DOACs) undergoing dental surgery. Int J Oral Maxillofac Surg 2016;45:618-630.

276. Mauprivez C, Khonsari RH, Razouk O, Goudot P, Lesclous P, Descroix V. Management of dental extraction in patients undergoing anticoagulant oral direct treatment: a pilot study. Oral Surg Oral Med Oral Pathol Oral Radiol 2016;122:e146-e155.

277. Patel JP, Woolcombe SA, Patel RK, Obisesan O, Roberts LN, Bryant C, Arya R. Managing direct oral anticoagulants in patients undergoing dentoalveolar surgery. Br Dent J 2017;222:245-249.

278. Yagyuu T, Kawakami M, Ueyama Y, Imada M, Kurihara M, Matsusue Y, Imai Y, Yamamoto K, Kirita T. Risks of postextraction bleeding after receiving direct oral anticoagulants or warfarin: a retrospective cohort study. BMJ Open 2017; 7:e015952

279. Miclotte I, Vanhaverbeke M, Agbaje JO, Legrand P, Vanassche T, Verhamme P, Politis C. Pragmatic approach to manage new oral anticoagulants in patients undergoing dental extractions: a prospective case-control study. Clin Oral Investig 2017;21:2183-2188.

280. Birnie DH, Healey JS, Wells GA, Verma A, Tang AS, Krahn AD, Simpson CS, Ayala-Paredes F, Coutu B, Leiria TL, Essebag V; BRUISE CONTROL Investigators. Pacemaker or defibrillator surgery without interruption of anticoagulation. N Engl J Med 2013;368:2084-2093.

281. Sticherling C, Marin F, Birnie D, Boriani G, Calkins H, Dan GA, Gulizia M, Halvorsen S, Hindricks G, Kuck KH, Moya A, Potpara T, Roldan V, Tilz R, Lip GY. Antithrombotic management in patients undergoing electrophysiological procedures: a European Heart Rhythm Association (EHRA) position document endorsed by the ESC Working Group Thrombosis, Heart Rhythm Society (HRS), and Asia Pacific Heart Rhythm Society (APHRS). Europace 2015;17:1197-1214.

282. Narouze S, Benzon HT, Provenzano DA, Buvanendran A, De Andres J, Deer TR, Rauck R, Huntoon MA. Interventional spine and pain procedures in patients on antiplatelet and anticoagulant medications: guidelines from the American Society of Regional Anesthesia and Pain Medicine, the European Society of Regional Anaesthesia and Pain Therapy, the American Academy of Pain Medicine, the International Neuromodulation Society, the North American Neuromodulation Society, and the World Institute of Pain. Reg Anesth Pain Med 2015;40:182-212.

283. Albaladejo P, Bonhomme F, Blais N, Collet JP, Faraoni D, Fontana P, Godier A, Llau J, Longrois D, Marret E, Mismetti P, Rosencher N, Roullet S, Samama CM, Schved JF, Sie P, Steib A, Susen S; French Working Group on Perioperative Hemostasis (GIHP). Management of direct oral anticoagulants in patients undergoing elective surgeries and invasive procedures: updated guidelines from the French Working Group on Perioperative Hemostasis (GIHP) - September 2015. Anaesth Crit Care Pain Med 2017;36:73-76.

284. Narouze S, Benzon HT, Provenzano D, Buvanendran A, De Andres J, Deer T, Rauck R, Huntoon MA. Interventional spine and pain procedures in patients on antiplatelet and anticoagulant medications (Second Edition): guidelines from the American Society of Regional Anesthesia and Pain Medicine, the European Society of Regional Anaesthesia and Pain Therapy, the American Academy of Pain Medicine, the International Neuromodulation Society, the North American Neuromodulation Society, and the World Institute of Pain. Reg Anesth Pain Med 2017; [Epub ahead of print].

285. Tripodi A. To measure or not to measure direct oral anticoagulants before surgery or invasive procedures: reply. J Thromb Haemost 2016;14:2559-2561.

286. Calkins H, Hindricks G, Cappato R, Kim Y-H, Saad EB, Aguinaga L, Akar JG, Badhwar V, Brugada J, Camm J, Chen P-S, Chen S-A, Chung MK, Nielsen JC, Curtis AB, Wyn Davies D, Day JD, D'Avila A, de Groot NMS, Di Biase L, Duytschaever M, Edgerton JR, Ellenbogen KA, Ellinor PT, Ernst S, Fenelon G, Gerstenfeld EP, Haines DE, Haissaguerre M, Helm RH, Hylek E, Jackman WM, Jalife J, Kalman JM, Kautzner J, Kottkamp H, Kuck KH, Kumagai K, Lee R, Lewalter T, Lindsay BD, Macle L, Mansour M, Marchlinski FE, Michaud GF, Nakagawa H, Natale A, Nattel S, Okumura K, Packer D, Pokushalov E, Reynolds MR, Sanders P, Scanavacca M, Schilling R, Tondo C, Tsao H-M, Verma A, Wilber DJ, Yamane T. 2017 HRS/EHRA/ECAS/APHRS/SOLAECE expert consensus statement on catheter and surgical ablation of atrial fibrillation: executive summary. J Interv Card Electrophysiol 2017;50:1-55.

287. Haeusler KG, Kirchhof $P$, Endres $M$. Left atrial catheter ablation and ischemic stroke. Stroke 2012;43:265-270.

288. Di Biase L, Burkhardt JD, Santangeli P, Mohanty P, Sanchez JE, Horton R, Gallinghouse G], Themistoclakis S, Rossillo A, Lakkireddy D, Reddy M, Hao S, Hongo R, Beheiry S, Zagrodzky J, Rong B, Mohanty S, Elayi CS, Forleo G, Pelargonio G, Narducci ML, Russo AD, Casella M, Fassini G, Tondo C Schweikert RA, Natale A. Periprocedural stroke and bleeding complications in patients undergoing catheter ablation of atrial fibrillation with different anticoagulation management: results from the role of coumadin in preventing thromboembolism in atrial fibrillation (AF) patients undergoing catheter ablation (COMPARE) randomized trial. Circulation 2014;129:2638-2644.

289. Calkins H, Willems S, Gerstenfeld EP, Verma A, Schilling R, Hohnloser SH, Okumura K, Serota H, Nordaby M, Guiver K, Biss B, Brouwer MA, Grimaldi M. Uninterrupted dabigatran versus warfarin for ablation in atrial fibrillation. N Engl J Med 2017;376:1627-1636. 
290. Cappato R, Marchlinski FE, Hohnloser SH, Naccarelli GV, Xiang J, Wilber DJ, Ma CS, Hess S, Wells DS, Juang G, Vijgen J, Hugl BJ, Balasubramaniam R, De Chillou C, Davies DW, Fields LE, Natale A; VENTURE-AF Investigators. Uninterrupted rivaroxaban vs. uninterrupted vitamin $\mathrm{K}$ antagonists for catheter ablation in non-valvular atrial fibrillation. Eur Heart J 2015;36:1805-1811.

291. Di Biase L, Callans D, Hæusler KG, Hindricks G, Al-Khalidi H, Mont L, Cosedis Nielsen J, Piccini JP, Schotten U, Kirchhof P. Rationale and design of AXAFAAFNET 5: an investigator-initiated, randomized, open, blinded outcome assessment, multi-centre trial to comparing continuous apixaban to vitamin $\mathrm{K}$ antagonists in patients undergoing atrial fibrillation catheter ablation. Europace 2017; 19:132-138.

292. Ukaigwe A, Shrestha P, Karmacharya P, Hussain SK, Samii S, Gonzalez MD, Wolbrette D, Naccarrelli GV. Meta-analysis of efficacy and safety of apixaban and uninterrupted apixaban therapy compared to vitamin $\mathrm{K}$ antagonists in patients undergoing catheter ablation for atrial fibrillation. J Interv Card Electrophysiol 2017;48:223-233.

293. Steffel J, Ruff CT, Hamershock RA, Murphy SA, Senior R, Roy D, Lanz HJ, Mercuri MF, Antman EM, Giugliano RP. First experience with edoxaban and atrial fibrillation ablation - Insights from the ENGAGE AF-TIMI 48 trial. Int $J$ Cardiol 2017;244:192-195.

294. Kottmaier M, Bourier F, Pausch H, Reents T, Semmler V, Telishevska M, KochButtner K, Lennerz C, Lengauer S, Kornmayer M, Rousseva E, Brooks S, Brkic A, Ammar-Busch S, Kaess B, Dillier R, Grebmer C, Kolb C, Hessling G, Deisenhofer I. Safety of uninterrupted periprocedural edoxaban versus phenprocoumon for patients who underwent left atrial catheter ablation procedures. Am J Cardiol 2018;121:445-449.

295. Torn M, Rosendaal FR. Oral anticoagulation in surgical procedures: risks and recommendations. Br J Haematol 2003;123:676-682.

296. Bassiouny M, Saliba W, Rickard J, Shao M, Sey A, Diab M, Martin DO, Hussein A, Khoury M, Abi-Saleh B, Alam S, Sengupta J, Borek PP, Baranowski B, Niebauer M, Callahan T, Varma N, Chung M, Tchou PJ, Kanj M, Dresing T, Lindsay $\mathrm{BD}$, Wazni $\mathrm{O}$. Use of dabigatran for periprocedural anticoagulation in patients undergoing catheter ablation for atrial fibrillation. Circ Arrhythm Electrophysiol 2013;6:460-466.

297. Di Biase L, Lakkireddy D, Trivedi C, Deneke T, Martinek M, Mohanty S, Mohanty P, Prakash S, Bai R, Reddy M, Gianni C, Horton R, Bailey S, Sigmund E, Derndorfer M, Schade A, Mueller P, Szoelloes A, Sanchez J, Al-Ahmad A, Hranitzky P, Gallinghouse G], Hongo RH, Beheiry S, Pürerfellner H, Burkhardt JD, Natale A. Feasibility and safety of uninterrupted periprocedural apixaban administration in patients undergoing radiofrequency catheter ablation for atrial fibrillation: results from a multicenter study. Heart Rhythm 2015;12: 1162-1168.

298. Death NCEiPOa. The NCEPOD Classification of Intervention. http://www.ncepod. org.uk/classification.html (8 March 2018).

299. Levy JH, Ageno W, Chan NC, Crowther M, Verhamme P, Weitz Jl; Subcommittee on Control of Anticoagulation. When and how to use antidotes for the reversal of direct oral anticoagulants: guidance from the SSC of the ISTH. J Thromb Haemost 2016;14:623-627.

300. Gwyn JCV, Thomas MR, Kirchhof P. Triple antithrombotic therapy in patients with atrial fibrillation undergoing percutaneous coronary intervention: a viewpoint. Eur Heart J Cardiovasc Pharmacother 2017;3:157-162.

301. Lopes RD, Li L, Granger CB, Wang TY, Foody JM, Funk M, Peterson ED, Alexander KP. Atrial fibrillation and acute myocardial infarction: antithrombotic therapy and outcomes. Am J Med 2012;125:897-905.

302. Alexander JH, Lopes RD, Thomas L, Alings M, Atar D, Aylward P, Goto S, Hanna M, Huber K, Husted S, Lewis BS, McMurray JJ, Pais P, Pouleur H, Steg PG, Verheugt FW, Wojdyla DM, Granger CB, Wallentin L. Apixaban vs. warfarin with concomitant aspirin in patients with atrial fibrillation: insights from the ARISTOTLE trial. Eur Heart J 2014;35:224-232.

303. Dewilde WJ, Oirbans T, Verheugt FW, Kelder JC, De Smet B], Herrman JP, Adriaenssens T, Vrolix M, Heestermans AA, Vis MM, Tijsen JG, van 't Hof AW, ten Berg JM; WOEST study investigators. Use of clopidogrel with or without aspirin in patients taking oral anticoagulant therapy and undergoing percutaneous coronary intervention: an open-label, randomised, controlled trial. Lancet 2013;381:1107-1115.

304. Rubboli A, Saia F, Sciahbasi A, Leone AM, Palmieri C, Bacchi-Reggiani ML, Calabro P, Bordoni B, Piccalo G, Franco N, Nicolino A, Magnavacchi P, Vignali L, Mameli S, Dallago M, Maggiolini S, Steffanon L, Piovaccari G, Di Pasquale G. Twelve-month outcome of patients with an established indication for oral anticoagulation undergoing coronary artery stenting and stratified by the baseline risk of bleeding: insights from the Warfarin and Coronary Stenting (War-Stent) Registry. Cardiovasc Revasc Med 2017;18:425-430.

305. Sra S, Tan MK, Mehta SR, Fisher HN, Dery JP, Welsh RC, Eisenberg MJ, Overgaard CB, Rose BF, Siega AJ, Cheema AN, Wong BY, Henderson MA, Lutchmedial S, Lavi S, Goodman SG, Yan AT. Ischemic and bleeding events in patients with myocardial infarction undergoing percutaneous coronary intervention who require oral anticoagulation: insights from the Canadian observational AntiPlatelet sTudy. Am Heart / 2016;180:82-89.

306. Lopes RD, Rao M, Simon DN, Thomas L, Ansell J, Fonarow GC, Gersh BJ, Go AS, Hylek EM, Kowey P, Piccini JP, Singer DE, Chang P, Peterson ED, Mahaffey $\mathrm{KW}$. Triple vs dual antithrombotic therapy in patients with atrial fibrillation and coronary artery disease. Am J Med 2016;129:592-599.e1.

307. Connolly S, Pogue J, Hart R, Pfeffer M, Hohnloser S, Chrolavicius S, Pfeffer M, Hohnloser S, Yusuf S. Clopidogrel plus aspirin versus oral anticoagulation for atrial fibrillation in the Atrial fibrillation Clopidogrel Trial with Irbesartan for prevention of Vascular Events (ACTIVE W): a randomised controlled trial. Lancet 2006:367:1903-1912.

308. Gibson CM, Mehran R, Bode C, Halperin J, Verheugt FW, Wildgoose P, Birmingham M, lanus J, Burton P, van Eickels M, Korjian S, Daaboul Y, Lip GY, Cohen M, Husted S, Peterson ED, Fox KA. Prevention of bleeding in patients with atrial fibrillation undergoing PCl. N Engl J Med 2016;375: 2423-2434.

309. Piccini JP, Jones WS. Triple therapy for atrial fibrillation after PCI. N Engl J Med 2017:377:1580-1582.

310. Vranckx P, Lewalter T, Valgimigli M, Tijssen JG, Reimitz P-E, Eckardt L, Lanz H-J, Zierhut W, Smolnik R, Goette A. Evaluation of the safety and efficacy of an edoxaban-based antithrombotic regimen in patients with atrial fibrillation following successful percutaneous coronary intervention $(\mathrm{PCl})$ with stent placement: rationale and design of the ENTRUST-AF PCl trial. Am Heart J 196:105-112.

311. Gibson CM, Mehran R, Bode C, Halperin J, Verheugt F, Wildgoose P, van Eickels M, Lip GY, Cohen M, Husted S, Peterson E, Fox K. An open-label, randomized, controlled, multicenter study exploring two treatment strategies of rivaroxaban and a dose-adjusted oral vitamin $\mathrm{K}$ antagonist treatment strategy in subjects with atrial fibrillation who undergo percutaneous coronary intervention (PIONEER AF-PCI). Am Heart J 2015;169:472-478.e5.

312. Oldgren J, Wallentin L, Alexander JH, James S, Jonelid B, Steg G, Sundstrom J. New oral anticoagulants in addition to single or dual antiplatelet therapy after an acute coronary syndrome: a systematic review and meta-analysis. Eur Heart J 2013;34:1670-1680.

313. Xu H, Ruff CT, Giugliano RP, Murphy SA, Nordio F, Patel I, Shi M, Mercuri M, Antman EM, Braunwald E. Concomitant use of single antiplatelet therapy with edoxaban or warfarin in patients with atrial fibrillation: analysis from the ENGAGE AF-TIMI48 Trial. J Am Heart Assoc 2016;5:e002587.

314. Urban P, Meredith IT, Abizaid A, Pocock SJ, Carrie D, Naber C, Lipiecki J, Richardt G, Iniguez A, Brunel P, Valdes-Chavarri M, Garot P, Talwar S, Berland J, Abdellaoui M, Eberli F, Oldroyd K, Zambahari R, Gregson J, Greene S, Stoll HP, Morice MC. Polymer-free drug-coated coronary stents in patients at high bleeding risk. N Engl J Med 2015;373:2038-2047.

315. Varenne O, Cook S, Sideris G, Kedev S, Cuisset T, Carrie D, Hovasse T, Garot P, El Mahmoud R, Spaulding C, Helft G, Diaz Fernandez JF, Brugaletta S, PinarBermudez E, Mauri Ferre J, Commeau P, Teiger E, Bogaerts K, Sabate M, Morice MC, Sinnaeve PR. Drug-eluting stents in elderly patients with coronary artery disease (SENIOR): a randomised single-blind trial. Lancet 2018;391:41-50.

316. Windecker S, Kolh P, Alfonso F, Collet JP, Cremer J, Falk V, Filippatos G, Hamm C, Head SJ, Juni P, Kappetein AP, Kastrati A, Knuuti J, Landmesser U, Laufer G, Neumann FJ, Richter DJ, Schauerte P, Sousa Uva M, Stefanini GG, Taggart DP, Torracca L, Valgimigli M, Wijns W, Witkowski A. 2014 ESC/ EACTS Guidelines on myocardial revascularization: the Task Force on Myocardial Revascularization of the European Society of Cardiology (ESC) and the European Association for Cardio-Thoracic Surgery (EACTS). Developed with the special contribution of the European Association of Percutaneous Cardiovascular Interventions (EAPCI). Eur Heart J 2014;35:2541-2619.

317. Bhatt DL, Flather MD, Hacke W, Berger PB, Black HR, Boden WE, Cacoub P, Cohen EA, Creager MA, Easton JD, Hamm CW, Hankey GJ, Johnston SC, Mak KH, Mas JL, Montalescot G, Pearson TA, Steg PG, Steinhubl SR, Weber MA, Fabry-Ribaudo L, Hu T, Topol EJ, Fox KA. Patients with prior myocardial infarction, stroke, or symptomatic peripheral arterial disease in the CHARISMA trial. J Am Coll Cardiol 2007;49:1982-1988.

318. Mauri L, Kereiakes DJ, Yeh RW, Driscoll-Shempp P, Cutlip DE, Steg PG, Normand S-LT, Braunwald E, Wiviott SD, Cohen DJ, Holmes DR, Krucoff MW, Hermiller J, Dauerman HL, Simon DI, Kandzari DE, Garratt KN, Lee DP, Pow TK, Ver Lee P, Rinaldi MJ, Massaro JM. Twelve or 30 months of dual antiplatelet therapy after drug-eluting stents. N Engl J Med 2014;371:2155-2166.

319. Bonaca MP, Bhatt DL, Cohen M, Steg PG, Storey RF, Jensen EC, Magnani G, Bansilal S, Fish MP, Im K, Bengtsson O, Oude Ophuis T, Budaj A, Theroux P, Ruda M, Hamm C, Goto S, Spinar J, Nicolau JC, Kiss RG, Murphy SA, Wiviott SD, Held P, Braunwald E, Sabatine MS. Long-term use of ticagrelor in patients with prior myocardial infarction. N Engl J Med 2015;372:1791-1800.

320. Karjalainen PP, Vikman S, Niemela M, Porela P, Ylitalo A, Vaittinen MA, Puurunen M, Airaksinen TJ, Nyman K, Vahlberg T, Airaksinen KE. Safety of 
percutaneous coronary intervention during uninterrupted oral anticoagulant treatment. Eur Heart J 2008;29:1001-1010.

321. Vranckx P, Verheugt FW, de Maat MP, Ulmans VA, Regar E, Smits P, ten Berg JM, Lindeboom W, Jones RL, Friedman J, Reilly P, Leebeek FW. A randomised study of dabigatran in elective percutaneous coronary intervention in stable coronary artery disease patients. Eurolntervention 2013;8:1052-1060.

322. Vranckx P, Leebeek FWG, Tijssen JGP, Koolen J, Stammen F, Herman J-PR, de Winter RJ, van T Hof AWJ, Backx B, Lindeboom W, Kim S-Y, Kirsch B, van Eickels M, Misselwitz F, Verheugt FWA. Peri-procedural use of rivaroxaban in elective percutaneous coronary intervention to treat stable coronary artery disease. The X-PLORER trial. Thromb Haemost 2015;114:258-267.

323. Ferguson JJ, Califf RM, Antman EM, Cohen M, Grines CL, Goodman S, Kereiakes DJ, Langer A, Mahaffey KW, Nessel CC, Armstrong PW, Avezum A, Aylward P, Becker RC, Biasucci L, Borzak S, Col J, Frey MJ, Fry E, Gulba DC, Guneri S, Gurfinkel E, Harrington R, Hochman JS, Kleiman NS, Leon MB, Lopez-Sendon JL, Pepine CJ, Ruzyllo W, Steinhubl SR, Teirstein PS, ToroFigueroa L, White H; SYNERGY Trial Investigators. Enoxaparin vs unfractionated heparin in high-risk patients with non-ST-segment elevation acute coronary syndromes managed with an intended early invasive strategy: primary results of the SYNERGY randomized trial. JAMA 2004;292:45-54.

324. Valgimigli M, Gagnor A, Calabró P, Frigoli E, Leonardi S, Zaro T, Rubartelli P, Briguori C, Andò G, Repetto A, Limbruno U, Cortese B, Sganzerla P, Lupi A, Galli M, Colangelo S, lerna S, Ausiello A, Presbitero P, Sardella G, Varbella F, Esposito G, Santarelli A, Tresoldi S, Nazzaro M, Zingarelli A, de Cesare N, Rigattieri S, Tosi P, Palmieri C, Brugaletta S, Rao SV, Heg D, Rothenbühler M, Vranckx P, Jüni P. Radial versus femoral access in patients with acute coronary syndromes undergoing invasive management: a randomised multicentre trial. Lancet 2015;385:2465-2476.

325. Lip GY, Windecker S, Huber K, Kirchhof P, Marin F, Ten Berg JM, Haeusler KG, Boriani G, Capodanno D, Gilard M, Zeymer U, Lane D, Document R, Storey RF, Bueno H, Collet JP, Fauchier L, Halvorsen S, Lettino M, Morais J, Mueller C, Potpara TS, Rasmussen LH, Rubboli A, Tamargo J, Valgimigli M, Zamorano JL. Management of antithrombotic therapy in atrial fibrillation patients presenting with acute coronary syndrome and/or undergoing percutaneous coronary or valve interventions: a joint consensus document of the European Society of Cardiology Working Group on Thrombosis, European Heart Rhythm Association (EHRA), European Association of Percutaneous Cardiovascular Interventions (EAPCl) and European Association of Acute Cardiac Care (ACCA) endorsed by the Heart Rhythm Society (HRS) and Asia-Pacific Heart Rhythm Society (APHRS). Eur Heart J 2014;35:3155-3179.

326. Povsic TJ, Roe MT, Ohman EM, Steg PG, James S, Plotnikov A, Mundl H, Welsh $\mathrm{R}$, Bode C, Gibson CM. A randomized trial to compare the safety of rivaroxaban vs aspirin in addition to either clopidogrel or ticagrelor in acute coronary syndrome: the design of the GEMINI-ACS-1 phase II study. Am Heart J 2016;174:120-128.

327. Roffi M, Patrono C, Collet JP, Mueller C, Valgimigli M, Andreotti F, Bax J, Borger MA, Brotons C, Chew DP, Gencer B, Hasenfuss G, Kjeldsen K, Lancellotti P, Landmesser U, Mehilli J, Mukherjee D, Storey RF, Windecker S, Baumgartner H, Gaemperli O, Achenbach S, Agewall S, Badimon L, Baigent C, Bueno H, Bugiardini R, Carerj S, Casselman F, Cuisset T, Erol C, Fitzsimons D, Halle M, Hamm C, Hildick-Smith D, Huber K, Iliodromitis E, James S, Lewis BS, Lip GY, Piepoli MF, Richter D, Rosemann T, Sechtem U, Steg PG, Vrints C, Luis Zamorano J. 2015 ESC Guidelines for the management of acute coronary syndromes in patients presenting without persistent ST-segment elevation: task Force for the Management of Acute Coronary Syndromes in Patients Presenting without Persistent ST-Segment Elevation of the European Society of Cardiology (ESC). Eur Heart J 2016;37:267-315.

328. Lamberts M, Olesen JB, Ruwald MH, Hansen CM, Karasoy D, Kristensen SL, Kober L, Torp-Pedersen C, Gislason GH, Hansen ML. Bleeding after initiation of multiple antithrombotic drugs, including triple therapy, in atrial fibrillation patients following myocardial infarction and coronary intervention: a nationwide cohort study. Circulation 2012;126:1185-1193.

329. Hohnloser SH, Oldgren J, Yang S, Wallentin L, Ezekowitz M, Reilly P, Eikelboom J, Brueckmann M, Yusuf S, Connolly SJ. Myocardial ischemic events in patients with atrial fibrillation treated with dabigatran or warfarin in the RELY (Randomized Evaluation of Long-Term Anticoagulation Therapy) trial. Circulation 2012;125:669-676.

330. Agnelli G, Buller HR, Cohen A, Curto M, Gallus AS, Johnson M, Masiukiewicz U, Pak R, Thompson J, Raskob GE, Weitz Jl; AMPLIFY Investigators. Oral apixaban for the treatment of acute venous thromboembolism. N Engl J Med 2013;369:799-808.

331. Schulman S, Kearon C, Kakkar AK, Mismetti P, Schellong S, Eriksson H, Baanstra D, Schnee J, Goldhaber SZ. Dabigatran versus warfarin in the treatment of acute venous thromboembolism. N Engl J Med 2009;361:2342-2352.

332. Hokusai VTEl, Buller HR, Decousus H, Grosso MA, Mercuri M, Middeldorp S, Prins MH, Raskob GE, Schellong SM, Schwocho L, Segers A, Shi M, Verhamme
P, Wells P. Edoxaban versus warfarin for the treatment of symptomatic venous thromboembolism. N Engl J Med 2013;369:1406-1415.

333. Bauersachs R, Berkowitz SD, Brenner B, Buller HR, Decousus H, Gallus AS, Lensing AW, Misselwitz F, Prins MH, Raskob GE, Segers A, Verhamme P, Wells P, Agnelli G, Bounameaux H, Cohen A, Davidson BL, Piovella F, Schellong S. Oral rivaroxaban for symptomatic venous thromboembolism. N Engl J Med 2010;363:2499-2510.

334. Buller HR, Prins MH, Lensin AW, Decousus H, Jacobson BF, Minar E, Chlumsky J, Verhamme P, Wells P, Agnelli G, Cohen A, Berkowitz SD, Bounameaux H, Davidson BL, Misselwitz F, Gallus AS, Raskob GE, Schellong S, Segers A. Oral rivaroxaban for the treatment of symptomatic pulmonary embolism. $N$ Engl Med 2012;366:1287-1297.

335. Agnelli G, Buller HR, Cohen A, Curto M, Gallus AS, Johnson M, Porcari A, Raskob GE, Weitz Jl; AMPLIFY-EXT Investigators. Apixaban for extended treatment of venous thromboembolism. N Engl J Med 2013;368:699-708.

336. Schulman S, Kearon C, Kakkar AK, Schellong S, Eriksson H, Baanstra D, Kvamme AM, Friedman J, Mismetti P, Goldhaber SZ; RE-MEDY Trial Investigators; RE-SONATE Trial Investigators. Extended use of dabigatran, warfarin, or placebo in venous thromboembolism. N Engl J Med 2013;368:709-718.

337. Weitz Jl, Lensing AWA, Prins MH, Bauersachs R, Beyer-Westendorf J, Bounameaux H, Brighton TA, Cohen AT, Davidson BL, Decousus H, Freitas MCS, Holberg G, Kakkar AK, Haskell L, van Bellen B, Pap AF, Berkowitz SD, Verhamme P, Wells PS, Prandoni P; EINSTEIN CHOICE Investigators. Rivaroxaban or aspirin for extended treatment of venous thromboembolism. N Engl J Med 2017;376:1211-1222.

338. Huang J, Cao Y, Liao C, Wu L, Gao F. Apixaban versus enoxaparin in patients with total knee arthroplasty. A meta-analysis of randomised trials. Thromb Haemost 2010;105:245.

339. Eriksson BI, Dahl OE, Huo MH, Kurth AA, Hantel S, Hermansson K, Schnee JM, Friedman RJ. Oral dabigatran versus enoxaparin for thromboprophylaxis after primary total hip arthroplasty (RE-NOVATE II). A randomised, double-blind, non-inferiority trial. Thromb Haemost 2011;105:721-729.

340. Eriksson BI, Dahl OE, Rosencher N, Kurth AA, VAN Dijk CN, Frostick SP, Kälebo P, Christiansen AV, Hantel S, Hettiarachchi R, Schnee J, Büller HR. Oral dabigatran etexilate vs. subcutaneous enoxaparin for the prevention of venous thromboembolism after total knee replacement: the RE-MODEL randomized trial. J Thromb Haemost 2007;5:2178-2185.

341. Fuji T, Wang C], Fujita S, Kawai Y, Nakamura M, Kimura T, Ibusuki K, Ushida $H$, Abe K, Tachibana S. Safety and efficacy of edoxaban, an oral factor $\mathrm{X}$ a inhibitor, versus enoxaparin for thromboprophylaxis after total knee arthroplasty: the STARS E-3 trial. Thromb Res 2014;134:1198-1204.

342. Fuji T, Fujita S, Kawai Y, Nakamura M, Kimura T, Fukuzawa M, Abe K, Tachibana S. Efficacy and safety of edoxaban versus enoxaparin for the prevention of venous thromboembolism following total hip arthroplasty: STARS J-V. Thromb J 2015;13:27.

343. Eriksson BI, Borris LC, Friedman RJ, Haas S, Huisman MV, Kakkar AK, Bandel TJ, Beckmann H, Muehlhofer E, Misselwitz F, Geerts W. Rivaroxaban versus enoxaparin for thromboprophylaxis after hip arthroplasty. N Engl J Med 2008;358:2765-2775.

344. Kakkar AK, Brenner B, Dahl OE, Eriksson BI, Mouret P, Muntz J, Soglian AG, Pap AF, Misselwitz F, Haas S. Extended duration rivaroxaban versus short-term enoxaparin for the prevention of venous thromboembolism after total hip arthroplasty: a double-blind, randomised controlled trial. Lancet 2008;372: 31-39.

345. Lassen MR, Ageno W, Borris LC, Lieberman JR, Rosencher N, Bandel TJ, Misselwitz F, Turpie AG. Rivaroxaban versus enoxaparin for thromboprophylaxis after total knee arthroplasty. N Engl J Med 2008;358:2776-2786.

346. Turpie AG, Lassen MR, Davidson BL, Bauer KA, Gent M, Kwong LM, Cushner FD, Lotke PA, Berkowitz SD, Bandel TJ, Benson A, Misselwitz F, Fisher WD. Rivaroxaban versus enoxaparin for thromboprophylaxis after total knee arthroplasty (RECORD4): a randomised trial. Lancet 2009;373:1673-1680.

347. Eikelboom JW, Connolly SJ, Bosch J, Dagenais GR, Hart RG, Shestakovska O Diaz R, Alings M, Lonn EM, Anand SS, Widimsky P, Hori M, Avezum A, Piegas LS, Branch KRH, Probstfield J, Bhatt DL, Zhu J, Liang Y, Maggioni AP, LopezJaramillo P, O'Donnell M, Kakkar AK, Fox KAA, Parkhomenko AN, Ertl G, Stork S, Keltai M, Ryden L, Pogosova N, Dans AL, Lanas F, Commerford PJ, Torp-Pedersen C, Guzik TJ, Verhamme PB, Vinereanu D, Kim JH, Tonkin AM, Lewis BS, Felix C, Yusoff K, Steg PG, Metsarinne KP, Cook Bruns N, Misselwitz F, Chen E, Leong D, Yusuf S; COMPASS Investigators. Rivaroxaban with or without aspirin in stable cardiovascular disease. $N$ Engl J Med 2017;377:1319-1330.

348. January CT, Wann LS, Alpert JS, Calkins H, Cigarroa JE, Cleveland JC Jr, Conti JB, Ellinor PT, Ezekowitz MD, Field ME, Murray KT, Sacco RL, Stevenson WG, Tchou PJ, Tracy CM, Yancy CW; American College of Cardiology/American Heart Association Task Force on Practice Guidelines. 2014 AHA/ACC/HRS guideline for the management of patients with atrial fibrillation: a report of the 
American College of Cardiology/American Heart Association Task Force on practice guidelines and the Heart Rhythm Society. Circulation 2014;130:e199-e267.

349. Cappato R, Ezekowitz MD, Klein AL, Camm AJ, Ma CS, Le Heuzey JY, Talajic M, Scanavacca M, Vardas PE, Kirchhof P, Hemmrich M, Lanius V, Meng IL, Wildgoose P, van Eickels M, Hohnloser SH; X-VeRT Investigators. Rivaroxaban vs. vitamin $\mathrm{K}$ antagonists for cardioversion in atrial fibrillation. Eur Heart J 2014:35:3346-3355.

350. Goette A, Merino JL, Ezekowitz MD, Zamoryakhin D, Melino M, Jin J, Mercuri MF, Grosso MA, Fernandez V, Al-Saady N, Pelekh N, Merkely B, Zenin S, Kushnir M, Spinar J, Batushkin V, de Groot JR, Lip GY. Edoxaban versus enoxaparin-warfarin in patients undergoing cardioversion of atrial fibrillation (ENSURE-AF): a randomised, open-label, phase 3b trial. Lancet 2016;388:1995-2003.

351. McCready JW, Nunn L, Lambiase PD, Ahsan SY, Segal OR, Rowland E, Lowe MD, Chow AW. Incidence of left atrial thrombus prior to atrial fibrillation ablation: is pre-procedural transoesophageal echocardiography mandatory? Europace 2010;12:927-932.

352. Puwanant S, Varr BC, Shrestha K, Hussain SK, Tang WH, Gabriel RS, Wazni OM, Bhargava M, Saliba WI, Thomas JD, Lindsay BD, Klein AL. Role of the CHADS2 score in the evaluation of thromboembolic risk in patients with atrial fibrillation undergoing transesophageal echocardiography before pulmonary vein isolation. J Am Coll Cardiol 2009;54:2032-2039.

353. Scherr D, Dalal D, Chilukuri K, Dong J, Spragg D, Henrikson CA, Nazarian S, Cheng A, Berger RD, Abraham TP, Calkins H, Marine JE. Incidence and predictors of left atrial thrombus prior to catheter ablation of atrial fibrillation. J Cardiovasc Electrophysiol 2009;20:379-384.

354. Ezekowitz MD, Pollack CV, Sanders P, Halperin JL, Spahr J, Cater N, Petkun W, Breazna A, Kirchhof P, Oldgren J. Apixaban compared with parenteral heparin and/or vitamin $\mathrm{K}$ antagonist in patients with nonvalvular atrial fibrillation undergoing cardioversion: rationale and design of the EMANATE trial. Am Heart J 2016;179:59-68.

355. Hansen ML, Jepsen RM, Olesen JB, Ruwald MH, Karasoy D, Gislason GH, Hansen J, Kober L, Husted S, Torp-Pedersen C. Thromboembolic risk in 16 274 atrial fibrillation patients undergoing direct current cardioversion with and without oral anticoagulant therapy. Europace 2015;17:18-23.

356. Gronberg T, Hartikainen JE, Nuotio I, Biancari F, Ylitalo A, Airaksinen KE. Anticoagulation, CHA2DS2VASc score, and thromboembolic risk of cardioversion of acute atrial fibrillation (from the FinCV Study). Am J Cardiol 2016;117:1294-1298.

357. Nuotio I, Hartikainen JE, Gronberg T, Biancari F, Airaksinen KE. Time to cardioversion for acute atrial fibrillation and thromboembolic complications. JAMA 2014;312:647-649.

358. Nagarakanti R, Ezekowitz MD, Oldgren J, Yang S, Chernick M, Aikens TH, Flaker G, Brugada J, Kamensky G, Parekh A, Reilly PA, Yusuf S, Connolly SJ. Dabigatran versus warfarin in patients with atrial fibrillation: an analysis of patients undergoing cardioversion. Circulation 2011;123:131-136.

359. Flaker G, Lopes RD, Al-Khatib SM, Hermosillo AG, Hohnloser SH, Tinga B, Zhu J, Mohan P, Garcia D, Bartunek J, Vinereanu D, Husted S, Harjola VP, Rosenqvist M, Alexander JH, Granger CB; ARISTOTLE Committees and Investigators. Efficacy and safety of apixaban in patients after cardioversion for atrial fibrillation: insights from the ARISTOTLE Trial (Apixaban for Reduction in Stroke and Other Thromboembolic Events in Atrial Fibrillation). I Am Coll Cardiol 2014;63:1082-1087.

360. Piccini JP, Stevens SR, Lokhnygina Y, Patel MR, Halperin JL, Singer DE, Hankey G], Hacke W, Becker RC, Nessel CC, Mahaffey KW, Fox KA, Califf RM, Breithardt G; ROCKET AF Steering Committee \& Investigators. Outcomes after cardioversion and atrial fibrillation ablation in patients treated with rivaroxaban and warfarin in the ROCKET AF trial. I Am Coll Cardiol 2013;61:1998-2006.

361. Lip GY, Hammerstingl C, Marin F, Cappato R, Meng IL, Kirsch B, van Eickels M, Cohen A, Study XT; X-TRA study and CLOT-AF registry investigators. Left atrial thrombus resolution in atrial fibrillation or flutter: results of a prospective study with rivaroxaban (X-TRA) and a retrospective observational registry providing baseline data (CLOT-AF). Am Heart J 2016;178:126-134.

362. Purrucker JC, Haas K, Rizos T, Khan S, Poli S, Kraft P, Kleinschnitz C, Dziewas $R$, Binder A, Palm F, Jander S, Soda $H$, Heuschmann PU, Veltkamp R; RASUNOA Investigators (Registry of Acute Stroke Under New Oral Anticoagulants). Coagulation Testing in acute ischemic stroke patients taking non-vitamin $\mathrm{K}$ antagonist oral anticoagulants. Stroke 2017;48:152-158.

363. Powers WJ, Rabinstein AA, Ackerson T, Adeoye OM, Bambakidis NC, Becker K, Biller J, Brown M, Demaerschalk BM, Hoh B, Jauch EC, Kidwell CS, LeslieMazwi TM, Ovbiagele B, Scott PA, Sheth KN, Southerland AM, Summers DV, Tirschwell DL; American Heart Association Stroke Council. 2018 guidelines for the early management of patients with acute ischemic stroke: a guideline for healthcare professionals from the American Heart Association/American Stroke Association. Stroke 2018; [Epub ahead of print].

364. Tse DM, Young L, Ranta A, Barber PA. Intravenous alteplase and endovascular clot retrieval following reversal of dabigatran with idarucizumab. I Neurol Neurosurg Psychiatry 2017; [Epub ahead of print].

365. Kermer P, Eschenfelder CC, Diener HC, Grond M, Abdalla Y, Althaus K, Berrouschot J, Cangur H, Daffertshofer M, Edelbusch S, Groschel K, Haase CG, Harloff A, Held V, Kauert A, Kraft P, Lenz A, Mullges W, Obermann M, Partowi S, Purrucker J, Ringleb PA, Rother J, Rossi R, Schafer N, Schneider A, Schuppner R, Seitz RJ, Szabo K, Wruck R. Antagonizing dabigatran by idarucizumab in cases of ischemic stroke or intracranial hemorrhage in Germany-a national case collection. Int J Stroke 2017;12:383-391.

366. Seiffge DI, Traenka C, Polymeris AA, Thilemann S, Wagner B, Hert L, Muller MD, Gensicke H, Peters N, Nickel CH, Stippich C, Sutter R, Marsch S, Fisch U Guzman R, De Marchis GM, Lyrer PA, Bonati LH, Tsakiris DA, Engelter ST. Intravenous thrombolysis in patients with stroke taking rivaroxaban using drug specific plasma levels: experience with a standard operation procedure in clinical practice. J Stroke 2017;19:347-355.

367. Xian Y, Federspiel J], Hernandez AF, Laskowitz DT, Schwamm LH, Bhatt DL, Smith EE, Fonarow GC, Peterson ED. Use of intravenous recombinant tissue plasminogen activator in patients with acute ischemic stroke who take nonvitamin $\mathrm{K}$ antagonist oral anticoagulants before stroke. Circulation 2017; 135:1024-1035

368. Ebner M, Birschmann I, Peter A, Hartig F, Spencer C, Kuhn J, Blumenstock G, Zuern CS, Ziemann U, Poli S. Emergency coagulation assessment during treatment with direct oral anticoagulants: limitations and solutions. Stroke 2017:48:2457-2463.

369. Ebner M, Birschmann I, Peter A, Spencer C, Hartig F, Kuhn J, Blumenstock G, Zuern CS, Ziemann U, Poli S. Point-of-care testing for emergency assessment of coagulation in patients treated with direct oral anticoagulants. Crit Care 2017:21:32.

370. Drouet L, Bal Dit Sollier C, Steiner T, Purrucker J. Measuring non-vitamin K antagonist oral anticoagulant levels: when is it appropriate and which methods should be used? Int J Stroke 2016;11:748-758.

371. Saver JL, Goyal M, van der Lugt A, Menon BK, Majoie CB, Dippel DW, Campbell BC, Nogueira RG, Demchuk AM, Tomasello A, Cardona P, Devlin TG, Frei DF, Du Mesnil de Rochemont R, Berkhemer OA, Jovin TG, Siddiqui AH, van Zwam WH, Davis SM, Castano C, Sapkota BL, Fransen PS, Molina C, van Oostenbrugge RJ, Chamorro A, Lingsma H, Silver FL, Donnan GA, Shuaib A, Brown S, Stouch B, Mitchell PJ, Davalos A, Roos YB, Hill MD; HERMES Collaborators. Time to treatment with endovascular thrombectomy and outcomes from ischemic stroke: a meta-analysis. JAMA 2016;316:1279-1288.

372. Nogueira RG, Jadhav AP, Haussen DC, Bonafe A, Budzik RF, Bhuva P, Yavagal DR, Ribo M, Cognard C, Hanel RA, Sila CA, Hassan AE, Millan M, Levy EI, Mitchell P, Chen M, English JD, Shah QA, Silver FL, Pereira VM, Mehta BP, Baxter BW, Abraham MG, Cardona P, Veznedaroglu E, Hellinger FR, Feng L, Kirmani JF, Lopes DK, Jankowitz BT, Frankel MR, Costalat V, Vora NA, Yoo AJ, Malik AM, Furlan AJ, Rubiera M, Aghaebrahim A, Olivot JM, Tekle WG, Shields R, Graves T, Lewis RJ, Smith WS, Liebeskind DS, Saver JL, Jovin TG. Thrombectomy 6 to 24 hours after stroke with a mismatch between deficit and infarct. N Engl J Med 2018;378:11-21.

373. Albers GW, Marks MP, Kemp S, Christensen S, Tsai JP, Ortega-Gutierrez S, McTaggart RA, Torbey MT, Kim-Tenser M, Leslie-Mazwi T, Sarraj A, Kasner SE, Ansari SA, Yeatts SD, Hamilton S, Mlynash M, Heit J], Zaharchuk G, Kim S, Carrozzella J, Palesch YY, Demchuk AM, Bammer R, Lavori PW, Broderick JP, Lansberg MG, Investigators D. Thrombectomy for stroke at 6 to 16 hours with selection by perfusion imaging. N Engl J Med 2018; [Epub ahead of print]

374. Wahlgren N, Moreira T, Michel P, Steiner T, Jansen O, Cognard C, Mattle HP, van Zwam W, Holmin S, Tatlisumak T, Petersson J, Caso V, Hacke W, Mazighi M, Arnold M, Fischer U, Szikora I, Pierot L, Fiehler J, Gralla J, Fazekas F, Lees KR; ESO-KSU, ESO, ESMINT, ESNR and EAN. Mechanical thrombectomy in acute ischemic stroke: consensus statement by ESO-Karolinska Stroke Update 2014/2015, supported by ESO, ESMINT, ESNR and EAN. Int J Stroke 2016;11:134-147.

375. Purrucker JC, Wolf M, Haas K, Rizos T, Khan S, Dziewas R, Kleinschnitz C, Binder A, Groschel K, Hennerici MG, Lobotesis K, Poli S, Seidel G, NeumannHaefelin T, Ringleb PA, Heuschmann PU, Veltkamp R. Safety of endovascular thrombectomy in patients receiving non-vitamin $\mathrm{K}$ antagonist oral anticoagulants. Stroke 2016:47:1127-1130

376. Hankey GJ. Intracranial hemorrhage and novel anticoagulants for atrial fibrillation: what have we learned? Curr Cardiol Rep 2014;16:480.

377. Lopes RD, Guimaraes PO, Kolls BJ, Wojdyla DM, Bushnell CD, Hanna M, Easton JD, Thomas L, Wallentin L, Al-Khatib SM, Held C, Gabriel Melo de Barros ESP, Alexander JH, Granger CB, Diener HC. Intracranial hemorrhage in patients with atrial fibrillation receiving anticoagulation therapy. Blood 2017:129:2980-2987. 
378. Wilson D, Seiffge DJ, Traenka C, Basir G, Purrucker JC, Rizos T, Sobowale OA, Sallinen H, Yeh SJ, Wu TY, Ferrigno M, Houben R, Schreuder F, Perry LA, Tanaka J, Boulanger M, Al-Shahi Salman R, Jager HR, Ambler G, Shakeshaft C, Yakushiji Y, Choi PMC, Staals J, Cordonnier C, Jeng JS, Veltkamp R, Dowlatshahi D, Engelter ST, Parry-Jones AR, Meretoja A. Werring DJ. Outcome of intracerebral hemorrhage associated with different oral anticoagulants. Neurology 2017;88:1693-1700.

379. Inohara T, Xian Y, Liang L, Matsouaka RA, Saver JL, Smith EE, Schwamm LH, Reeves MJ, Hernandez AF, Bhatt DL, Peterson ED, Fonarow GC. Association of intracerebral hemorrhage among patients taking non-vitamin $\mathrm{K}$ antagonist vs vitamin $\mathrm{K}$ antagonist oral anticoagulants with in-hospital mortality. JAMA 2018;319:463-473.

380. Hemphill JC, Greenberg SM, Anderson CS, Becker K, Bendok BR, Cushman M, Fung GL, Goldstein JN, Macdonald RL, Mitchell PH, Scott PA, Selim MH, Woo D. Guidelines for the management of spontaneous intracerebral hemorrhage: a guideline for healthcare professionals from the American Heart Association/ American Stroke Association. Stroke 2015;46:2032-2060.

381. Kuramatsu JB, Gerner ST, Schellinger PD, Glahn J, Endres M, Sobesky J, Flechsenhar J, Neugebauer H, Juttler E, Grau A, Palm F, Rother J, Michels P, Hamann GF, Huwel J, Hagemann G, Barber B, Terborg C, Trostdorf F, Bazner H, Roth A, Wohrle J, Keller M, Schwarz M, Reimann G, Volkmann J, Mullges W, Kraft P, Classen J, Hobohm C, Horn M, Milewski A, Reichmann H, Schneider H, Schimmel E, Fink GR, Dohmen C, Stetefeld H, Witte O, Gunther A, NeumannHaefelin T, Racs AE, Nueckel M, Erbguth F, Kloska SP, Dorfler A, Kohrmann M, Schwab S, Huttner HB. Anticoagulant reversal, blood pressure levels, and anticoagulant resumption in patients with anticoagulation-related intracerebral hemorrhage. JAMA 2015;313:824-836.

382. Gerner ST, Kuramatsu JB, Sembill JA, Sprugel MI, Endres M, Haeusler KG, Vajkoczy P, Ringleb PA, Purrucker J, Rizos T, Erbguth F, Schellinger PD, Fink GR, Stetefeld H, Schneider H, Neugebauer H, Rother J, Classen J, Michalski D, Dorfler A, Schwab S, Huttner HB, Investigators RI. Association of prothrombin complex concentrate administration and hematoma enlargement in non-vitamin $\mathrm{K}$ antagonist oral anticoagulant-related intracerebral hemorrhage. Ann Neurol 2018;83:186-196.

383. Paciaroni M, Agnelli G, Falocci N, Tsivgoulis G, Vadikolias K, Liantinioti C, Chondrogianni M, Bovi P, Carletti M, Cappellari M, Zedde M, Ntaios G, Karagkiozi E, Athanasakis G, Makaritsis K, Silvestrelli G, Lanari A, Ciccone A, Putaala J, Tomppo L, Tatlisumak T, Abdul-Rahim AH, Lees KR, Alberti A, Venti M, Acciarresi M, D'Amore C, Becattini C, Mosconi MG, Cimini LA, Soloperto R, Masotti L, Vannucchi V, Lorenzini G, Tassi R, Guideri F, Acampa M, Martini G, Sohn SI, Marcheselli S, Mumoli N, De Lodovici ML, Bono G, Furie KL, Tadi P, Yaghi S, Toni D, Letteri F, Tassinari T, Kargiotis O, Lotti EM, Flomin Y, Mancuso M, Maccarrone M, Giannini N, Bandini F, Pezzini A, Poli L, Padovani A, Scoditti U, Denti L, Consoli D, Galati F, Sacco S, Carolei A, Tiseo C, Gourbali V, Orlandi G, Giuntini M, Chiti A, Giorli E, Gialdini G, Corea F, Ageno W, Bellesini M, Colombo G, Monaco S, Maimone Baronello M, Karapanayiotides T, Caso V. Early recurrence and major bleeding in patients with acute ischemic stroke and atrial fibrillation treated with non-vitamin-K oral anticoagulants (RAF-NOACs) study. J Am Heart Assoc 2017;6:e007034.

384. Ahmed N, Steiner T, Caso V, Wahlgren N. Recommendations from the ESOKarolinska Stroke Update Conference, Stockholm 13-15 November 2016. Eur Stroke J 2017;2:95-102.

385. Paciaroni M, Agnelli G, Falocci N, Caso V, Becattini C, Marcheselli S, Rueckert C, Pezzini A, Poli L, Padovani A, Csiba L, Szabó L, Sohn S-I, Tassinari T, AbdulRahim AH, Michel P, Cordier M, Vanacker P, Remillard S, Alberti A, Venti M, Scoditti U, Denti L, Orlandi G, Chiti A, Gialdini G, Bovi P, Carletti M, Rigatelli A, Putaala J, Tatlisumak T, Masotti L, Lorenzini G, Tassi R, Guideri F, Martini G, Tsivgoulis G, Vadikolias K, Liantinioti C, Corea F, Del Sette M, Ageno W, De Lodovici ML, Bono G, Baldi A, D'Anna S, Sacco S, Carolei A, Tiseo C, Acciarresi M, D'Amore C, Imberti D, Zabzuni D, Doronin B, Volodina V, Consoli D, Galati F, Pieroni A, Toni D, Monaco S, Baronello MM, Barlinn K, Pallesen L-P, Kepplinger J, Bodechtel U, Gerber J, Deleu D, Melikyan G, Ibrahim F, Akhtar N, Mosconi MG, Bubba V, Silvestri I, Lees KR. Early recurrence and cerebral bleeding in patients with acute ischemic stroke and atrial fibrillation: effect of anticoagulation and its timing: the RAF study. Stroke 2015;46:2175-2182.

386. Paciaroni M, Agnelli G, Micheli S, Caso V. Efficacy and safety of anticoagulant treatment in acute cardioembolic stroke: a meta-analysis of randomized controlled trials. Stroke 2007;38:423-430.

387. Orrapin S, Rerkasem K. Carotid endarterectomy for symptomatic carotid stenosis. Cochrane Database Syst Rev 2017;6:CD001081.

388. Brønnum Nielsen P, Larsen TB, Gorst-Rasmussen A, Skjøth F, Rasmussen LH, Lip GYH. Intracranial hemorrhage and subsequent ischemic stroke in patients with atrial fibrillation: a nationwide cohort study. Chest 2015;147:1651-1658.

389. Korompoki E, Filippidis FT, Nielsen PB, Del Giudice A, Lip GYH, Kuramatsu JB, Huttner HB, Fang J, Schulman S, Marti-Fabregas J, Gathier CS, Viswanathan A, Biffi A, Poli D, Weimar C, Malzahn U, Heuschmann P, Veltkamp R. Long-term antithrombotic treatment in intracranial hemorrhage survivors with atrial fibrillation. Neurology 2017;89:687-696.

390. Banerjee G, Carare R, Cordonnier C, Greenberg SM, Schneider JA, Smith EE, Buchem MV, Grond JV, Verbeek MM, Werring DJ. The increasing impact of cerebral amyloid angiopathy: essential new insights for clinical practice. J Neurol Neurosurg Psychiatry 2017;88:982-994.

391. Biffi A, Kuramatsu JB, Leasure A, Kamel H, Kourkoulis C, Schwab K, Ayres AM, Elm J, Gurol ME, Greenberg SM, Viswanathan A, Anderson CD, Schwab S, Rosand J, Testai FD, Woo D, Huttner HB, Sheth KN. Oral anticoagulation and functional outcome after intracerebral hemorrhage. Ann Neurol 2017;82:755-765.

392. Rietbrock S, Heeley E, Plumb J, van Staa T. Chronic atrial fibrillation: incidence, prevalence, and prediction of stroke using the Congestive heart failure, Hypertension, Age $>75$, Diabetes mellitus, and prior Stroke or transient ischemic attack (CHADS2) risk stratification scheme. Am Heart J 2008;156:57-64.

393. Wolff A, Shantsila E, Lip GY, Lane DA. Impact of advanced age on management and prognosis in atrial fibrillation: insights from a population-based study in general practice. Age Ageing 2015;44:874-878.

394. Wolf PA, Abbott RD, Kannel WB. Atrial fibrillation as an independent risk factor for stroke: the Framingham Study. Stroke 1991;22:983-988.

395. Fohtung RB, Novak E, Rich MW. Effect of new oral anticoagulants on prescribing practices for atrial fibrillation in older adults. I Am Geriatr Soc 2017;65:2405-2412.

396. Bai Y, Guo SD, Deng H, Shantsila A, Fauchier L, Ma CS, Lip GYH. Effectiveness and safety of oral anticoagulants in older patients with atrial fibrillation: a systematic review and meta-regression analysis. Age Ageing 2018;47:9-17.

397. Fumagalli S, Potpara TS, Bjerregaard Larsen T, Haugaa KH, Dobreanu D, Proclemer A, Dagres N. Frailty syndrome: an emerging clinical problem in the everyday management of clinical arrhythmias. The results of the European Heart Rhythm Association survey. Europace 2017;19:1896-1902.

398. Wehling M, Collins R, Gil VM, Hanon O, Hardt R, Hoffmeister M, Monteiro P, Quinn TJ, Ropers D, Sergi G, Verheugt FWA. Appropriateness of oral anticoagulants for the long-term treatment of atrial fibrillation in older people: results of an evidence-based review and international consensus validation process (OAC-FORTA 2016). Drugs Aging 2017;34:499-507.

399. Kato ET, Giugliano RP, Ruff CT, Koretsune Y, Yamashita T, Kiss RG, Nordio F, Murphy SA, Kimura T, Jin J, Lanz H, Mercuri M, Braunwald E, Antman EM. Efficacy and safety of edoxaban in elderly patients with atrial fibrillation in the ENGAGE AF-TIMI 48 Trial. J Am Heart Assoc 2016;5:e003432.

400. Halperin JL, Hankey GJ, Wojdyla DM, Piccini JP, Lokhnygina Y, Patel MR, Breithardt G, Singer DE, Becker RC, Hacke W, Paolini JF, Nessel CC, Mahaffey KW, Califf RM, Fox KA; ROCKET AF Steering Committee and Investigator. Efficacy and safety of rivaroxaban compared with warfarin among elderly patients with nonvalvular atrial fibrillation in the Rivaroxaban Once Daily, Oral, Direct Factor $\mathrm{Xa}$ Inhibition Compared With Vitamin K Antagonism for Prevention of Stroke and Embolism Trial in Atrial Fibrillation (ROCKET AF). Circulation 2014;130:138-146.

401. Halvorsen S, Atar D, Yang H, De Caterina R, Erol C, Garcia D, Granger CB, Hanna M, Held C, Husted S, Hylek EM, Jansky P, Lopes RD, Ruzyllo W, Thomas L, Wallentin L. Efficacy and safety of apixaban compared with warfarin according to age for stroke prevention in atrial fibrillation: observations from the ARISTOTLE trial. Eur Heart J 2014;35:1864-1872.

402. Okumura K, Lip GYH, Akao M, Tanizawa K, Fukuzawa M, Abe K, Akishita M, Yamashita T. Edoxaban for the management of elderly Japanese patients with atrial fibrillation ineligible for standard oral anticoagulant therapies: rationale and design of the ELDERCARE-AF study. Am Heart J 2017;194:99-106.

403. Fried LP, Tangen CM, Walston J, Newman AB, Hirsch C, Gottdiener J, Seeman T, Tracy R, Kop WJ, Burke G, McBurnie MA; Cardiovascular Health Study Collaborative Research Group. Frailty in older adults: evidence for a phenotype. J Gerontol A Biol Sci Med Sci 2001;56:M146-M156.

404. Rockwood K, Stadnyk K, MacKnight C, McDowell I, Hebert R, Hogan DB. A brief clinical instrument to classify frailty in elderly people. Lancet 1999;353:205-206.

405. Rockwood K, Song X, MacKnight C, Bergman H, Hogan DB, McDowell I, Mitnitski A. A global clinical measure of fitness and frailty in elderly people. CMA/ 2005;173:489-495.

406. Rubenstein LZ. Falls in older people: epidemiology, risk factors and strategies for prevention. Age Ageing 2006;35(Suppl 2):ii37-ii41.

407. Hylek EM, D’Antonio J, Evans-Molina C, Shea C, Henault LE, Regan S. Translating the results of randomized trials into clinical practice: the challenge of warfarin candidacy among hospitalized elderly patients with atrial fibrillation. Stroke 2006:37:1075-1080.

408. Man-Son-Hing M, Nichol G, Lau A, Laupacis A. Choosing antithrombotic therapy for elderly patients with atrial fibrillation who are at risk for falls. Arch Intern Med 1999;159:677-685.

409. Rao MP, Vinereanu D, Wojdyla DM, Alexander JH, Atar D, Hylek EM, Hanna M, Wallentin L, Lopes RD, Gersh BJ, Granger CB; Apixaban for Reduction in 
Stroke Other Thromboembolic Events in Atrial Fibrillation (ARISTOTLE) Investigators. Clinical outcomes and history of fall in patients with atrial fibrillation treated with oral anticoagulation: insights from the ARISTOTLE trial. Am J Med 2018;131:269-275.

410. Tiedemann A, Lord SR, Sherrington C. The development and validation of a brief performance-based fall risk assessment tool for use in primary care. J Gerontol A Biol Sci Med Sci 2010;65:896-903.

411. Hanon O, Assayag P, Belmin J, Collet JP, Emeriau JP, Fauchier L, Forette F, Friocourt P, Gentric A, Leclercq C, Komajda M, Le Heuzey JY; French Society of Geriatrics, Gerantology, French Society of Cardiology. Expert consensus of the French Society of Geriatrics and Gerontology and the French Society of Cardiology on the management of atrial fibrillation in elderly people. Arch Cardiovasc Dis 2013;106:303-323.

412. Sherrington C, Whitney JC, Lord SR, Herbert RD, Cumming RG, Close JC. Effective exercise for the prevention of falls: a systematic review and meta-analysis. J Am Geriatr Soc 2008;56:2234-2243.

413. Tricco AC, Thomas SM, Veroniki AA, Hamid JS, Cogo E, Strifler L, Khan PA, Robson R, Sibley KM, MacDonald H, Riva JJ, Thavorn K, Wilson C, HolroydLeduc J, Kerr GD, Feldman F, Majumdar SR, Jaglal SB, Hui W, Straus SE. Comparisons of interventions for preventing falls in older adults: a systematic review and meta-analysis. JAMA 2017;318:1687-1699.

414. Garcia-Ptacek S, Contreras Escamez B, Zupanic E, Religa D, von Koch L, Johnell K, von Euler M, Kåreholt I, Eriksdotter M. Prestroke mobility and dementia as predictors of stroke outcomes in patients over 65 years of age: a cohort study from the Swedish Dementia and Stroke Registries. J Am Med Dir Assoc 2018;19:154-161.

415. Friberg L, Rosenqvist M. Less dementia with oral anticoagulation in atrial fibrillation. Eur Heart J 2018;39:453-460.

416. Dietzel J, Haeusler KG, Endres M. Does atrial fibrillation cause cognitive decline and dementia? Europace 2018;20:408.

417. World Health Organization. Obesity and Overweight Fact Sheet. http://www.who. int/mediacentre/factsheets/ss311/en/ (8 March 2018).

418. Wang TJ, Parise H, Levy D, D’Agostino RB Sr, Wolf PA, Vasan RS, Benjamin EJ. Obesity and the risk of new-onset atrial fibrillation. JAMA 2004;292:2471-2477.

419. Lavie CJ, Pandey A, Lau DH, Alpert MA, Sanders P. Obesity and atrial fibrillation prevalence, pathogenesis, and prognosis: effects of weight loss and exercise. J Am Coll Cardiol 2017;70:2022-2035.

420. Sivasambu B, Balouch MA, Zghaib T, Bajwa RJ, Chrispin J, Berger RD, Ashikaga $\mathrm{H}$, Nazarian S, Marine JE, Calkins H, Spragg DD. Increased rates of atrial fibrillation recurrence following pulmonary vein isolation in overweight and obese patients. J Cardiovasc Electrophysiol 2018;29:239-245.

421. Abed HS, Wittert GA, Leong DP, Shirazi MG, Bahrami B, Middeldorp ME, Lorimer MF, Lau DH, Antic NA, Brooks AG, Abhayaratna WP, Kalman JM, Sanders $\mathrm{P}$. Effect of weight reduction and cardiometabolic risk factor management on symptom burden and severity in patients with atrial fibrillation: a randomized clinical trial. JAMA 2013;310:2050-2060.

422. Chagnac A, Weinstein T, Korzets A, Ramadan E, Hirsch J, Gafter U. Glomerular hemodynamics in severe obesity. Am J Physiol Renal Physiol 2000;278:F817-F822.

423. Wallace JL, Reaves AB, Tolley EA, Oliphant CS, Hutchison L, Alabdan NA, Sands CW, Self TH. Comparison of initial warfarin response in obese patients versus non-obese patients. J Thromb Thrombolysis 2013;36:96-101.

424. Kubitza D, Becka M, Zuehlsdorf M, Mueck W. Body weight has limited influence on the safety, tolerability, pharmacokinetics, or pharmacodynamics of rivaroxaban (BAY 59-7939) in healthy subjects. J Clin Pharmacol 2007;47:218-226.

425. Upreti VV, Wang J, Barrett YC, Byon W, Boyd RA, Pursley J, LaCreta FP, Frost CE. Effect of extremes of body weight on the pharmacokinetics, pharmacodynamics, safety and tolerability of apixaban in healthy subjects. $\mathrm{Br} J \mathrm{Clin}$ Pharmacol 2013;76:908-916.

426. Yin OQ, Tetsuya K, Miller R. Edoxaban population pharmacokinetics and exposure-response analysis in patients with non-valvular atrial fibrillation. Eur J Clin Pharmacol 2014;70:1339-1351.

427. Martin K, Beyer-Westendorf J, Davidson BL, Huisman MV, Sandset PM, Moll S. Use of the direct oral anticoagulants in obese patients: guidance from the SSC of the ISTH. J Thromb Haemost 2016;14:1308-1313.

428. Burnett AE, Mahan CE, Vazquez SR, Oertel LB, Garcia DA, Ansell J. Guidance for the practical management of the direct oral anticoagulants (DOACs) in VTE treatment. J Thromb Thrombolysis 2016;41:206-232.

429. Breuer L, Ringwald J, Schwab S, Kohrmann M. Ischemic stroke in an obese patient receiving dabigatran. N Engl J Med 2013;368:2440-2442.

430. Safouris A, Demulder A, Triantafyllou N, Tsivgoulis G. Rivaroxaban presents a better pharmacokinetic profile than dabigatran in an obese non-diabetic stroke patient. J Neurol Sci 2014;346:366-367.

431. Sandhu RK, Ezekowitz J, Andersson U, Alexander JH, Granger CB, Halvorsen S, Hanna M, Hijazi Z, Jansky P, Lopes RD, Wallentin L. The 'obesity paradox' in atrial fibrillation: observations from the ARISTOTLE (Apixaban for Reduction in
Stroke and Other Thromboembolic Events in Atrial Fibrillation) trial. Eur Heart J 2016;37:2869-2878.

432. Balla SR, Cyr DD, Lokhnygina Y, Becker RC, Berkowitz SD, Breithardt G, Fox KAA, Hacke W, Halperin JL, Hankey GJ, Mahaffey KW, Nessel CC, Piccini JP, Singer DE, Patel MR. Relation of risk of stroke in patients with atrial fibrillation to body mass index (from Patients Treated With Rivaroxaban and Warfarin in the Rivaroxaban Once Daily Oral Direct Factor Xa Inhibition Compared with Vitamin K Antagonism for Prevention of Stroke and Embolism Trial in Atrial Fibrillation Trial). Am J Cardiol 2017;119:1989-1996.

433. Braekkan SK, van der Graaf Y, Visseren FL, Algra A. Obesity and risk of bleeding: the SMART study. J Thromb Haemost 2016;14:65-72.

434. Lee CH, Lin TY, Chang SH, Chen CH, Hsu YJ, Hung KC, Wen MS. Body mass index is an independent predictor of major bleeding in non-valvular atrial fibrillation patients taking dabigatran. Int J Cardiol 2017;228:771-778.

435. De Caterina R, Lip GYH. The non-vitamin $\mathrm{K}$ antagonist oral anticoagulants (NOACs) and extremes of body weight-a systematic literature review. Clin Res Cardiol 2017; 106:565-572.

436. Van Eijkeren MA, Christiaens GC, Sixma J], Haspels AA. Menorrhagia: a review. Obstet Gynecol Surv 1989;44:421-429.

437. Huq FY, Tvarkova K, Arafa A, Kadir RA. Menstrual problems and contraception in women of reproductive age receiving oral anticoagulation. Contraception 2011;84:128-132

438. De Crem N, Peerlinck K, Vanassche T, Vanheule K, Debaveye B, Middeldorp S, Verhamme $P$, Peetermans M. Abnormal uterine bleeding in VTE patients treated with rivaroxaban compared to vitamin $\mathrm{K}$ antagonists. Thromb Res 2015;136:749-753.

439. Martinelli I, Lensing AW, Middeldorp S, Levi M, Beyer-Westendorf J, van Bellen B, Bounameaux H, Brighton TA, Cohen AT, Trajanovic M, Gebel M, Lam P. Wells PS, Prins MH. Recurrent venous thromboembolism and abnormal uterine bleeding with anticoagulant and hormone therapy use. Blood 2016;127:1417-1425.

440. Beyer-Westendorf J, Michalski F, Tittl L, Hauswald-Dörschel S, Marten S. Management and outcomes of vaginal bleeding and heavy menstrual bleeding in women of reproductive age on direct oral anti-factor $\mathrm{Xa}$ inhibitor therapy: a case series. Lancet Haematol 2016;3:e480-e488.

441. Rolden HJA, Maas A, van der Wilt GJ, Grutters JPC. Uncertainty on the effectiveness and safety of rivaroxaban in premenopausal women with atrial fibrillation: empirical evidence needed. BMC Cardiovasc Disord 2017;17:260.

442. Tobenkin A, Mohamoud M, Munoz M, Waldron P. Direct oral anticoagulants and menorrhagia in premenopausal women: data from the food and drug administration adverse event reporting system and US drug utilization. Haemophilia 2017;23(Suppl 3):43.

443. Nielsen JR, Wachtell K, Abdulla J. The relationship between physical activity and risk of atrial fibrillation-a systematic review and meta-analysis. J Atr Fibrillation 2013;5:789.

444. Zhu WG, Wan R, Din Y, Xu Z, Yang X, Hong K. Sex differences in the association between regular physical activity and incident atrial fibrillation: a metaanalysis of 13 prospective studies. Clin Cardiol 2016;39:360-367.

445. Kwok CS, Anderson SG, Myint PK, Mamas MA, Loke YK. Physical activity and incidence of atrial fibrillation: a systematic review and meta-analysis. Int J Cardiol 2014; 177:467-476.

446. Heidbuchel H, Anne W, Willems R, Adriaenssens B, Van de Werf F, Ector H. Endurance sports is a risk factor for atrial fibrillation after ablation for atrial flutter. Int J Cardiol 2006;107:67-72.

447. Caselli S, Vaquer Sequi A, Lemme E, Quattrini F, Milan A, D’Ascenzi F, Spataro A, Pelliccia A. Prevalence and Management of Systemic Hypertension in Athletes. Am J Cardiol 2017;119:1616-1622.

448. Stefanidou M, Das RR, Beiser AS, Sundar B, Kelly-Hayes M, Kase CS, Devinsky O, Seshadri S, Friedman D. Incidence of seizures following initial ischemic stroke in a community-based cohort: the Framingham Heart Study. Seizure 2017;47:105-110

449. Beghi E, D'Alessandro R, Beretta S, Consoli D, Crespi V, Delaj L, Gandolfo C Greco G, La Neve A, Manfredi M, Mattana F, Musolino R, Provinciali L, Santangelo M, Specchio LM, Zaccara G, Epistroke G. Incidence and predictors of acute symptomatic seizures after stroke. Neurology 2011;77:1785-1793.

450. Hesdorffer DC, Benn EK, Cascino GD, Hauser WA. Is a first acute symptomatic seizure epilepsy? Mortality and risk for recurrent seizure. Epilepsia 2009;50:1102-1108.

451. Hu YF, Liu CJ, Chang PM, Tsao HM, Lin YJ, Chang SL, Lo LW, Tuan TC, Li CH, Chao TF, Chung FP, Liao JN, Chen TJ, Chen SA. Incident thromboembolism and heart failure associated with new-onset atrial fibrillation in cancer patients. Int J Cardiol 2013;165:355-357.

452. Onaitis M, D'Amico T, Zhao Y, O'Brien S, Harpole D. Risk factors for atrial fibrillation after lung cancer surgery: analysis of the Society of Thoracic Surgeons general thoracic surgery database. Ann Thorac Surg 2010;90:368-374. 
453. Guglin M, Aljayeh M, Saiyad S, Ali R, Curtis AB. Introducing a new entity: chemotherapy-induced arrhythmia. Europace 2009;11:1579-1586.

454. Farmakis D, Parissis J, Filippatos G. Insights into onco-cardiology: atrial fibrillation in cancer. I Am Coll Cardiol 2014;63:945-953.

455. Horsted F, West J, Grainge MJ. Risk of venous thromboembolism in patients with cancer: a systematic review and meta-analysis. PLoS Med 2012;9:e1001275.

456. Caine GJ, Stonelake PS, Lip GY, Kehoe ST. The hypercoagulable state of malignancy: pathogenesis and current debate. Neoplasia 2002;4:465-473.

457. Lenneman CG, Sawyer DB. Cardio-oncology: an update on cardiotoxicity of cancer-related treatment. Circ Res 2016;118:1008-1020.

458. Raskob GE, van Es N, Verhamme P, Carrier M,D, Nisio M, Garcia D, Grosso MA, Kakkar AK, Kovacs MJ, Mercuri MF, Meyer G, Segers A, Shi M, Wang TF, Yeo E, Zhang G, Zwicker Jl, Weitz Jl, Buller HR; Hokusai VTE Cancer Investigators. Edoxaban for the treatment of cancer-associated venous thromboembolism. N Engl J Med 2018;378:615-624.

459. Posch F, Konigsbrugge O, Zielinski C, Pabinger I, Ay C. Treatment of venous thromboembolism in patients with cancer: a network meta-analysis comparing efficacy and safety of anticoagulants. Thromb Res 2015;136:582-589.

460. Brunetti ND, Gesuete E, De Gennaro L, Correale M, Caldarola P, Gaglione A, Di Biase M. Direct oral anti-coagulants compared with vitamin-K inhibitors and low-molecular-weight-heparin for the prevention of venous thromboembolism in patients with cancer: a meta-analysis study. Int J Cardiol 2017;230:214-221.

461. Melloni C, Dunning A, Granger CB, Thomas L, Khouri MG, Garcia DA, Hylek EM, Hanna M, Wallentin L, Gersh BJ, Douglas PS, Alexander JH, Lopes RD. Efficacy and safety of apixaban versus warfarin in patients with atrial fibrillation and a history of cancer: insights from the ARISTOTLE Trial. Am J Med 2017;130:1440-1448.

462. Ording AG, Horváth-Puhó E, Adelborg K, Pedersen L, Prandoni P, Sørensen HT. Thromboembolic and bleeding complications during oral anticoagulation therapy in cancer patients with atrial fibrillation: a Danish nationwide population-based cohort study. Cancer Med 2017;6:1165-1172.

463. Zamorano JL, Lancellotti P, Rodriguez Munoz D, Aboyans V, Asteggiano R, Galderisi M, Habib G, Lenihan DJ, Lip GY, Lyon AR, Lopez Fernandez T, Mohty D, Piepoli MF, Tamargo J, Torbicki A, Suter TM; Authors/Task Force Members; ESC Committee for Practice Guidelines(CPG); Document Reviewers. 2016 ESC Position Paper on cancer treatments and cardiovascular toxicity developed under the auspices of the ESC Committee for Practice Guidelines: the Task Force for cancer treatments and cardiovascular toxicity of the European Society of Cardiology (ESC). Eur Heart J 2016;37:2768-2801.

464. Zhang Y, de Boer A, Verhoef TI, van der Meer FJM, Le Cessie S, Maitland-van der Zee AH, Barallon R, de Boer A, Daly A, Maitland-van der Zee A-H, Redekop K, Stingl J, Manolopoulos VG, Rosendaal FR, Wadelius M. Comparison of dosing algorithms for acenocoumarol and phenprocoumon using clinical factors with the standard care in the Netherlands. Thromb Res 2015;136:94-100.

465. Stergiopoulos K, Brown DL. Genotype-guided vs clinical dosing of warfarin and its analogues: meta-analysis of randomized clinical trials. JAMA Intern Med 2014;174:1330-1338.
466. Kovacs MJ, Rodger M, Anderson DR, Morrow B, Kells G, Kovacs J, Boyle E, Wells PS. Comparison of 10-mg and 5-mg warfarin initiation nomograms together with low-molecular-weight heparin for outpatient treatment of acute venous thromboembolism. A randomized, double-blind, controlled trial. Ann Intern Med 2003;138:714-719.

467. Garcia P, Ruiz W, Loza Munarriz C. Warfarin initiation nomograms for venous thromboembolism. Cochrane Database Syst Rev 2016;1:CD007699.

468. Belley-Cote EP, Hanif H, D'Aragon F, Eikelboom JW, Anderson JL, Borgman M, Jonas DE, Kimmel SE, Manolopoulos VG, Baranova E, Maitland-van der Zee $\mathrm{AH}$, Pirmohamed M, Whitlock RP. Genotype-guided versus standard vitamin K antagonist dosing algorithms in patients initiating anticoagulation. A systematic review and meta-analysis. Thromb Haemost 2015;114:768-777.

469. van Schie RM, Wessels JA, Le Cessie S, de Boer A, Schalekamp T, van der Meer FJ, Verhoef TI, van Meegen E, Rosendaal FR, Maitland-van der Zee AH; EUPACT Study Group. Loading and maintenance dose algorithms for phenprocoumon and acenocoumarol using patient characteristics and pharmacogenetic data. Eur Heart J 2011;32:1909-1917.

470. Mahtani KR, Heneghan CJ, Nunan D, Bankhead C, Keeling D, Ward AM, Harrison SE, Roberts NW, Hobbs FD, Perera R. Optimal loading dose of warfarin for the initiation of oral anticoagulation. Cochrane Database Syst Rev 2012;12:CD008685.

471. Jones M, McEwan P, Morgan CL, Peters JR, Goodfellow J, Currie CJ. Evaluation of the pattern of treatment, level of anticoagulation control, and outcome of treatment with warfarin in patients with non-valvar atrial fibrillation: a record linkage study in a large British population. Heart 2005;91:472-477.

472. Van Spall HG, Wallentin L, Yusuf S, Eikelboom JW, Nieuwlaat R, Yang S, Kabali C, Reilly PA, Ezekowitz MD, Connolly SJ. Variation in warfarin dose adjustment practice is responsible for differences in the quality of anticoagulation control between centers and countries: an analysis of patients receiving warfarin in the randomized evaluation of long-term anticoagulation therapy (RE-LY) trial. Circulation 2012;126:2309-2316.

473. Wallentin L, Yusuf S, Ezekowitz MD, Alings M, Flather M, Franzosi MG, Pais P, Dans A, Eikelboom J, Oldgren J, Pogue J, Reilly PA, Yang S, Connolly SJ, Investigators R. L. Efficacy and safety of dabigatran compared with warfarin at different levels of international normalised ratio control for stroke prevention in atrial fibrillation: an analysis of the RE-LY trial. Lancet 2010;376:975-983.

474. Fitzmaurice DA, Hobbs FD, Murray ET, Holder RL, Allan TF, Rose PE. Oral anticoagulation management in primary care with the use of computerized decision support and near-patient testing: a randomized, controlled trial. Arch Intern Med 2000;160:2343-2348.

475. Wilson SJ, Wells PS, Kovacs MJ, Lewis GM, Martin J, Burton E, Anderson DR. Comparing the quality of oral anticoagulant management by anticoagulation clinics and by family physicians: a randomized controlled trial. CMAJ 2003;169:293-298.

476. Heneghan CJ, Garcia-Alamino JM, Spencer EA, Ward AM, Perera R, Bankhead C, Alonso-Coello P, Fitzmaurice D, Mahtani KR, Onakpoya IJ. Self-monitoring and self-management of oral anticoagulation. Cochrane Database Syst Rev 2016; 7:CD003839. 